Nevada

Environmental

Restoration

Project

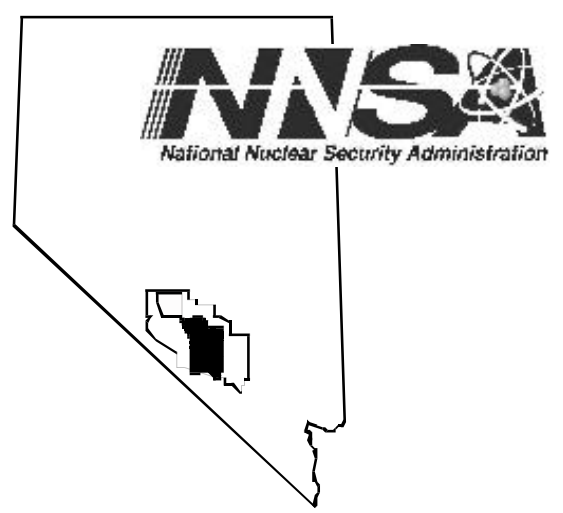

\title{
Completion Report for Well Cluster ER-5-4
}

February 2005

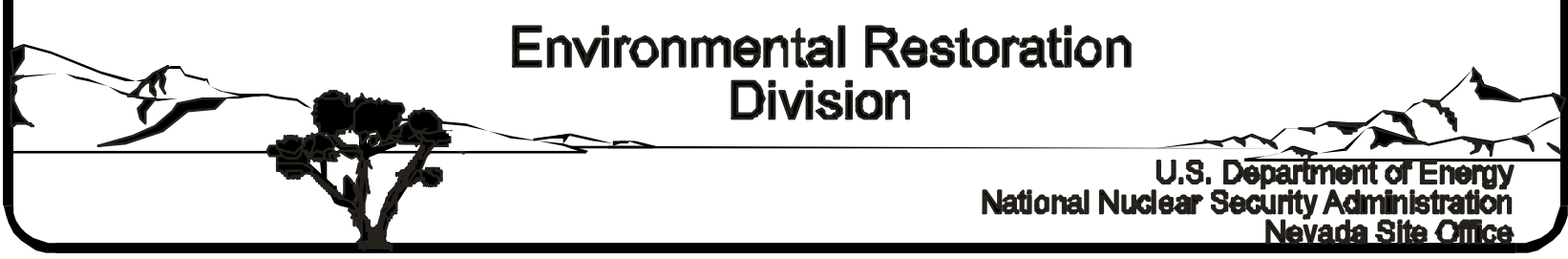




\section{DISCLAIMER STATEMENT}

Reference herein to any specific commercial product, process, or service by trade name, trademark, manufacturer, or otherwise, does not necessarily constitute or imply its endorsement, recommendation, or favoring by the U.S. Government or any agency thereof or its contractors or subcontractors.

\section{AVAILABILITY STATEMENT}

Available for sale to the public from-

U.S. Department of Commerce

National Technical Information Service

5285 Port Royal Road

Springfield, VA, 22161-0002

Telephone: 800.553 .6847

Fax: 703.605.6900

E-mail: orders@ntis.gov

Online ordering: http://www.ntis.gov/ordering.htm

Available electronically at http://www.osti.gov/bridge.

Available for a processing fee to U.S. Department of Energy and its contractors, in paper, from-

U.S. Department of Energy

Office of Scientific and Technical Information

P.O. Box 62

Oak Ridge, TN 37831-0062

Telephone: 865.576 .8401

Fax: 865.576.5728

E-mail: reports@adonis.osti.gov 


\title{
Completion Report for Well Cluster ER-5-4
}

\author{
Prepared for: \\ U.S. Department of Energy \\ National Nuclear Security Administration \\ Nevada Site Office \\ Las Vegas, Nevada
}

Prepared by:

Bechtel Nevada

Geotechnical Sciences

Las Vegas, Nevada

February 2005 
This page intentionally left blank. 


\section{COMPLETION REPORT FOR WELL CLUSTER ER-5-4}

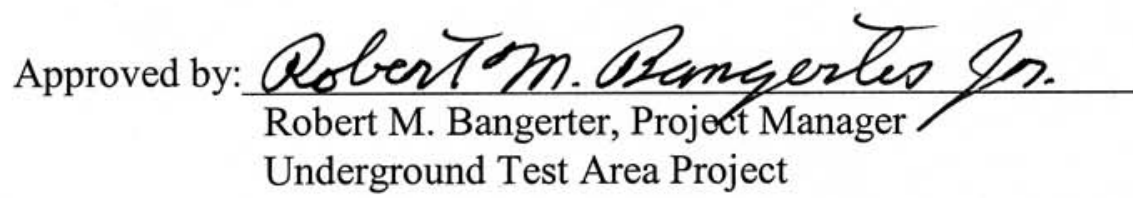

Date: $3 / 8 / 05$

Underground Test Area Project

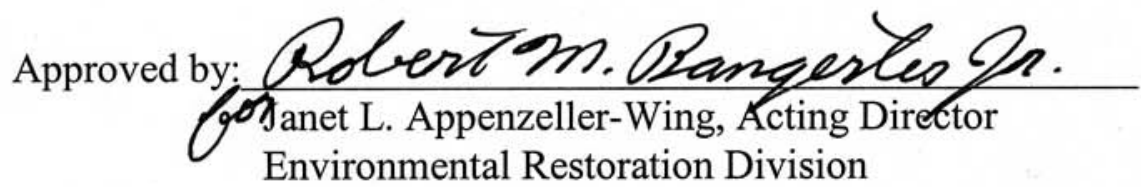

Date: $3 / 8 / 05$ 
This page intentionally left blank. 


\title{
Completion Report for Well Cluster ER-5-4 \\ DOE/NV/11718--998
}

\begin{abstract}
Well Cluster ER-5-4 was drilled for the U.S. Department of Energy, National Nuclear Security Administration Nevada Site Office, in support of the Nevada Environmental Restoration Project at the Nevada Test Site, Nye County, Nevada. The cluster consists of two wells, positioned about 30 meters apart on the same drill pad, constructed as part of a hydrogeologic investigation program for Frenchman Flat at the Nevada Test Site.

At Well ER-5-4, constructed in early 2001, a 66.0-centimeter surface hole was drilled and cased off to a depth of 279.1 meters below the surface. The hole diameter was then decreased to 44.5 centimeters and cased off to a depth of 510.0 meters. The borehole size was further decreased to 31.1 centimeters for drilling to a total depth of 1,115.6 meters. At Well ER-5-4\#2, constructed in mid-2002, a 66.0-centimeter surface hole was drilled and cased off to a depth of 266.5 meters. The hole diameter was decreased and the borehole cased off twice more, with the final casing set at the depth of 1,477.9 meters. Borehole size was decreased again to 22.2 centimeters for drilling to a total depth of 2,133.6 meters. Severe problems with borehole instability were encountered during drilling of both holes, which required use of bentonite drill fluid, the use of lost-circulation materials, and cementing and redrilling parts of the holes.
\end{abstract}

At Well ER-5-4, a 7.3-centimeter piezometer string with one slotted interval was installed in the annulus of the surface casing. A 14.0-centimeter completion string with 2 isolated slotted intervals was also installed in the well. All 3 completions are open to the alluvial aquifer. A preliminary composite, static water level was measured at the depth of 221.3 meters.

At Well ER-5-4\#2, a string of 14.0-centimeter stainless-steel casing hangs from a liner hanger set at 1,437.1 meters within 24.4-centimeter carbon-steel intermediate casing. The bottom of the completion string is at the depth of 2,030.0 meters, and the casing is slotted in the interval 1,976.9 to 2,029.3 meters, open to the tuff confining unit. No gravel-pack or cement was utilized.

Detailed lithologic descriptions with preliminary stratigraphic assignments for the well cluster are included in this report. These are based on composite drill cuttings collected every 3 meters, and 156 sidewall samples taken at various depths below 192 meters in both boreholes, supplemented by geophysical log data. Detailed petrographic, chemical, and mineralogical studies of rock samples were conducted on 122 samples. Well ER-5-4 penetrated approximately 1,120 meters of Quaternary and Tertiary alluvium before reaching total depth in Tertiary volcanic rocks at 1,137.5 meters. The deeper Well ER-5-4\#2 penetrated 1,120.4 meters of alluvial sediments, and was terminated within Tertiary volcanic rocks at a depth of 2,133.6 meters, indicating that Paleozoic rocks are deeper than expected at this site. 
This page intentionally left blank. 


\section{Table of Contents}

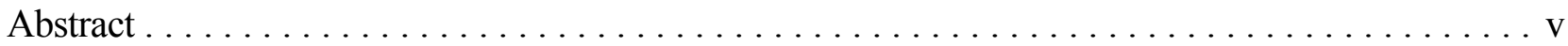

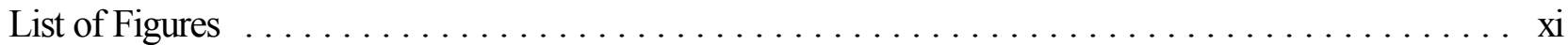

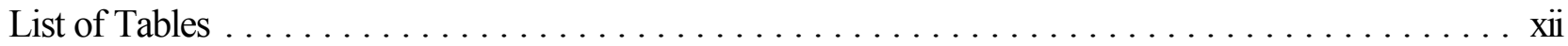

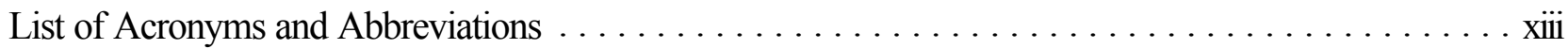

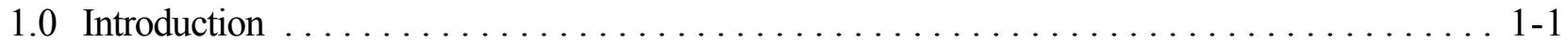

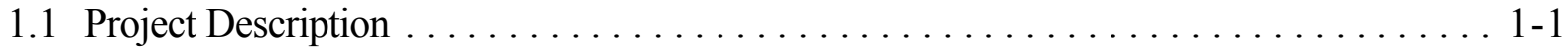

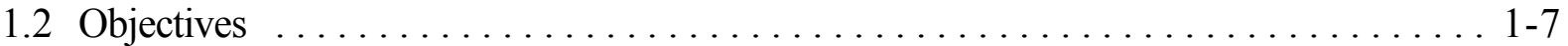

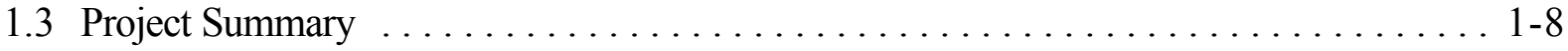

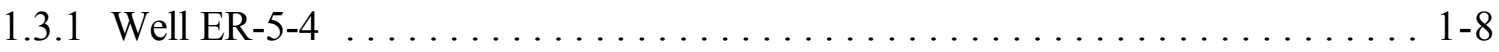

1.3.2 Well ER-5-4\#2 ................................. 1-9

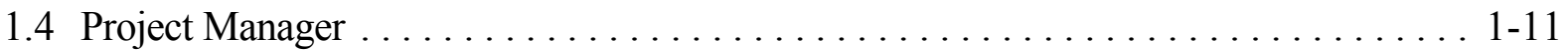

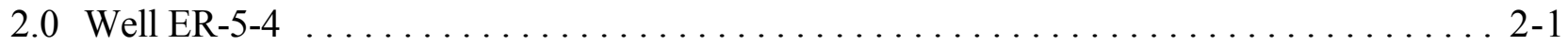

2.1 Well-Specific Objectives .................................. 2-1

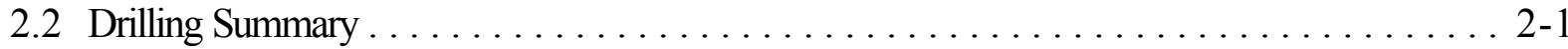

2.2 .1 Introduction $\ldots \ldots \ldots \ldots \ldots \ldots \ldots \ldots \ldots \ldots \ldots \ldots \ldots \ldots \ldots \ldots \ldots \ldots \ldots \ldots \ldots \ldots, 1$

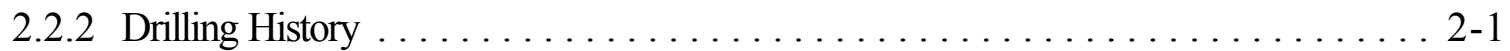

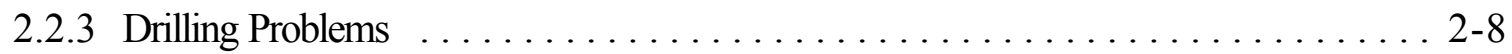

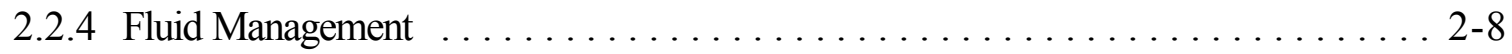

2.3 Geologic Data Collection ................................. 2-10

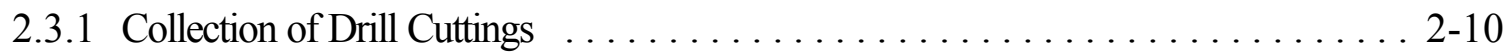

2.3.2 Sidewall Core Samples ................................ 2-11

2.3.3 Sample Analysis . .................................... 2-11

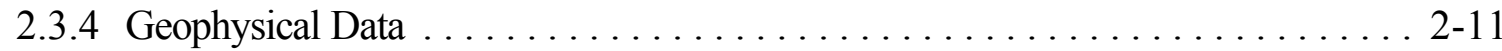

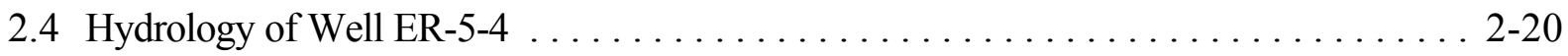

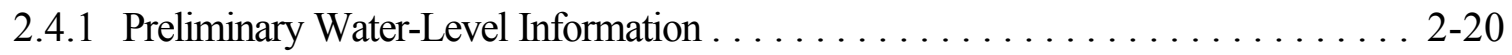

2.4.2 Water Production .................................. 2-20

2.4.3 Preliminary Flow Meter and Chemistry Log Data $\ldots \ldots \ldots \ldots \ldots \ldots .2-21$

2.4.4 Preliminary Groundwater Characterization Samples ................ 2-21

2.5 Precompletion and Open-Hole Development . . . . . . . . . . . . . . . . . . . . 2-21 


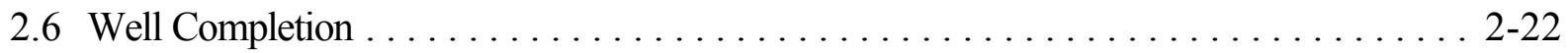

2.6.1 Well Completion Design .............................. 2-22

2.6.1.1 Proposed Completion Design $\ldots \ldots \ldots \ldots \ldots \ldots \ldots \ldots \ldots \ldots \ldots .2-22$

2.6.1.2 As-Built Completion Design ............................ 2-26

2.6.1.3 Rationale for Differences between Actual and Proposed Well Design . . . . . . . . . . . . . . . . . . . . . . 2-27

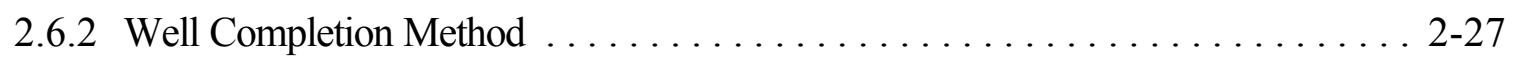

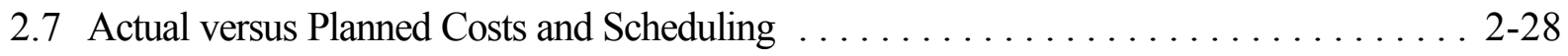

2.8 Summary, Recommendations, and Lessons Learned $\ldots \ldots \ldots \ldots \ldots \ldots \ldots \ldots \ldots$

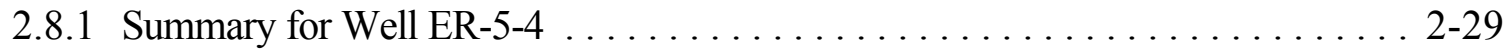

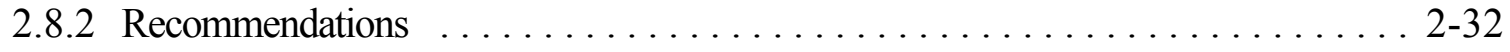

2.8.3 Lessons Learned from Well ER-5-4 ..................... 2-32

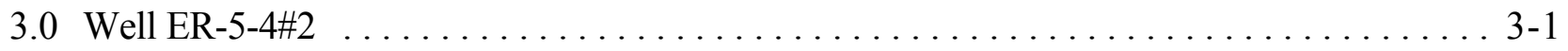

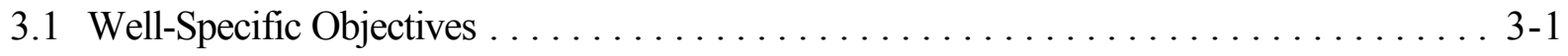

3.2 Drilling Summary $\ldots \ldots \ldots \ldots \ldots \ldots \ldots \ldots \ldots \ldots \ldots \ldots \ldots \ldots \ldots \ldots \ldots \ldots \ldots \ldots \ldots \ldots, 1$

3.2 .1 Introduction $\ldots \ldots \ldots \ldots \ldots \ldots \ldots \ldots \ldots \ldots \ldots \ldots \ldots \ldots \ldots \ldots \ldots \ldots \ldots \ldots, 1$

3.2 .2 Drilling History $\ldots \ldots \ldots \ldots \ldots \ldots \ldots \ldots \ldots \ldots \ldots \ldots \ldots \ldots \ldots \ldots \ldots \ldots \ldots \ldots, 1$

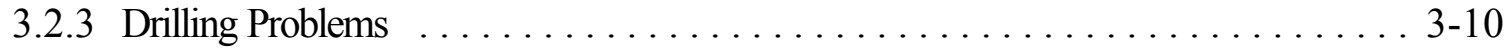

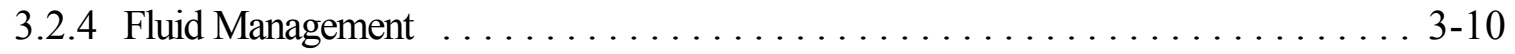

3.3 Geologic Data Collection ................................... 3-11

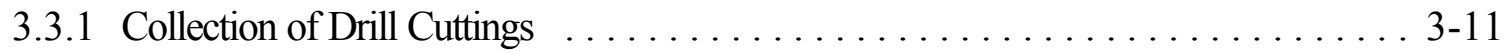

3.3.2 Sidewall Core Samples ................................ 3-12

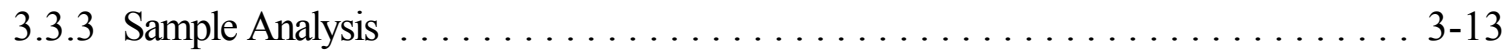

3.3.4 Geophysical Data ................................ 3-13

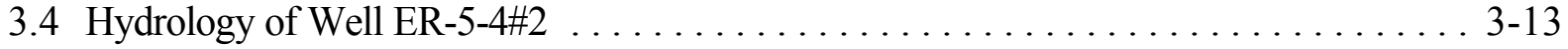

3.4.1 Preliminary Water-Level Information $\ldots \ldots \ldots \ldots \ldots \ldots \ldots \ldots \ldots \ldots \ldots \ldots \ldots \ldots \ldots$

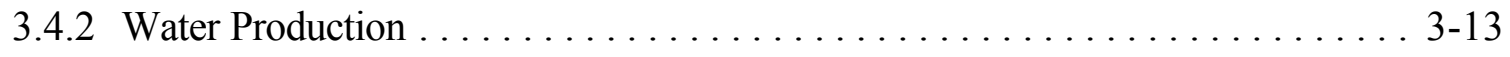

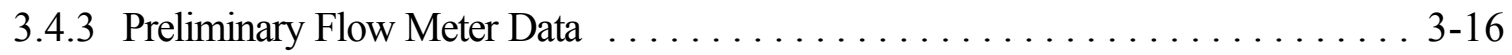

3.4.4 Preliminary Groundwater Characterization Samples . . . . . . . . . . . . . . 3-17

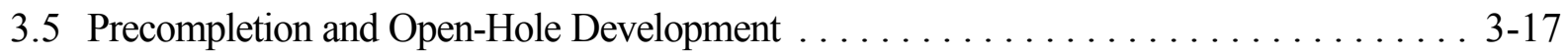




\section{Table of Contents (Continued)}

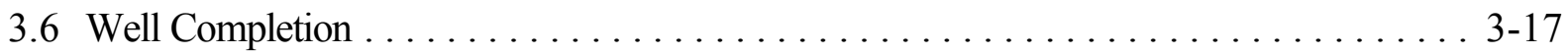

3.6.1 Well Completion Design ............................. 3-17

3.6.1.1 Proposed Completion Design ........................... 3-20

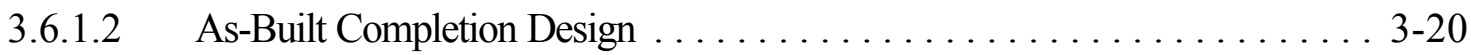

3.6.1.3 Rationale for Differences between Actual and Proposed

Well Design ............................... 3-21

3.6.2 Well Completion Method ............................ 3-21

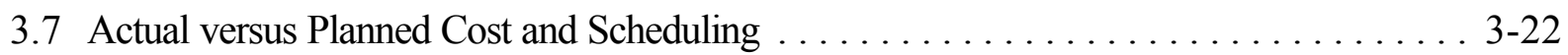

3.8 Summary, Recommendations, and Lessons Learned $\ldots \ldots \ldots \ldots \ldots \ldots \ldots \ldots . . \ldots \ldots$

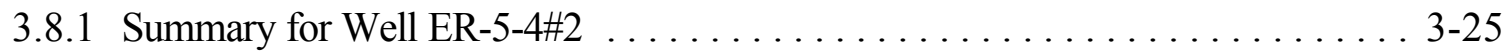

3.8.2 Recommendations $\ldots \ldots \ldots \ldots \ldots \ldots \ldots \ldots \ldots \ldots \ldots \ldots \ldots \ldots \ldots \ldots \ldots \ldots, 25$

3.8.3 Lessons Learned from Well ER-5-4\#2 $\ldots \ldots \ldots \ldots \ldots \ldots \ldots \ldots \ldots \ldots . \ldots \ldots$

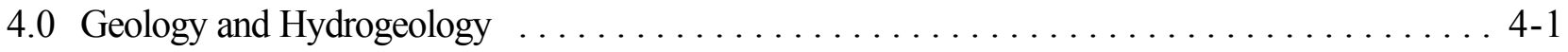

4.1 Introduction $\ldots \ldots \ldots \ldots \ldots \ldots \ldots \ldots \ldots \ldots \ldots \ldots \ldots \ldots \ldots, \ldots \ldots \ldots \ldots \ldots, 1$

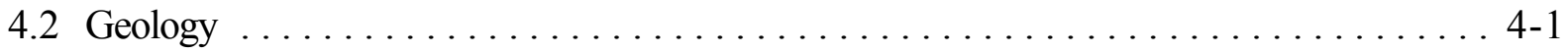

4.2 .1 Geologic Setting $\ldots \ldots \ldots \ldots \ldots \ldots \ldots \ldots \ldots \ldots \ldots \ldots \ldots \ldots \ldots \ldots \ldots \ldots \ldots \ldots \ldots \ldots, 1$

4.2 .2 Stratigraphy and Lithology $\ldots \ldots \ldots \ldots \ldots \ldots \ldots \ldots \ldots \ldots \ldots \ldots \ldots \ldots \ldots \ldots$

4.2.2.1 Well ER-5-4 ........................... 4-6

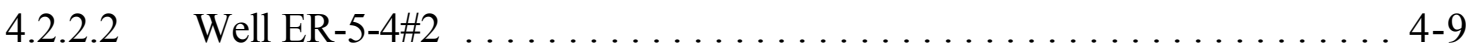

4.2 .3 Alteration $\ldots \ldots \ldots \ldots \ldots \ldots \ldots \ldots \ldots \ldots \ldots \ldots \ldots \ldots \ldots \ldots, 4-12$

4.3 Predicted Versus Actual Geology $\ldots \ldots \ldots \ldots \ldots \ldots \ldots \ldots \ldots \ldots \ldots . . \ldots \ldots .12$

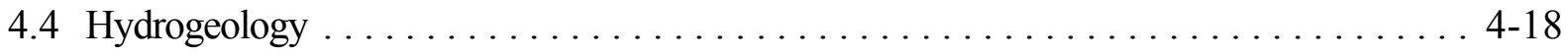

5.0 Summary, Recommendations, and Lessons Learned $\ldots \ldots \ldots \ldots \ldots \ldots \ldots \ldots \ldots \ldots$. 5-1

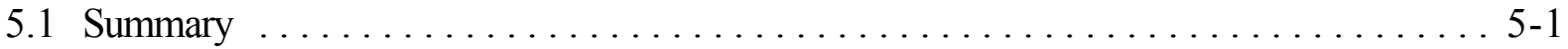

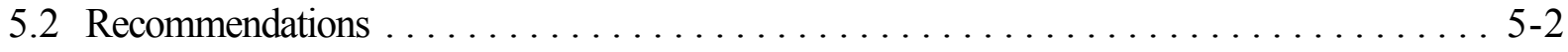

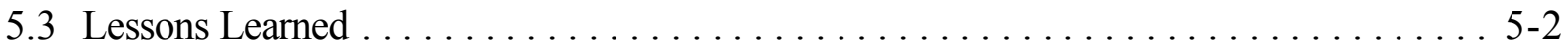

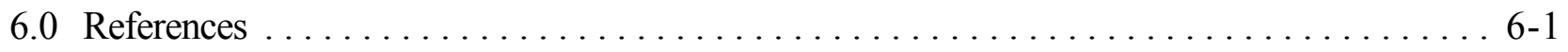

Appendix A - Drilling Data

A-1 Drilling Parameter Logs for Well Cluster ER-5-4

A-2 Casing and Tubing Data for Well Cluster ER-5-4

A-3 Well Cluster ER-5-4 Drilling Fluids and Cement Composition 
Appendix B - Well Cluster ER-5-4 Fluid Management Data

Well ER-5-4 Fluid Disposition Reporting Form

Well ER-5-4\#2 Fluid Disposition Reporting Form

Preliminary Analytical Results for Fluid Management Samples from Well ER-5-4

Appendix C - Lithologic Logs for Well Cluster ER-5-4

C-1 Detailed Lithologic Log for Well ER-5-4

C-2 Detailed Lithologic Log for Well ER-5-4\#2

Appendix D - Geophysical Logs Run at Well Cluster ER-5-4

D-1 Geophysical Logs Run in Well ER-5-4

D-2 Geophysical Logs Run in Well ER-5-4-\#2

Distribution List 


\section{List of Figures}

Number

Title

Page

1-1 Reference Map Showing Location of Well Cluster ER-5-4 . . . . . . . . . . . . . . 1-3

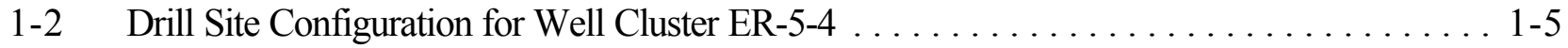

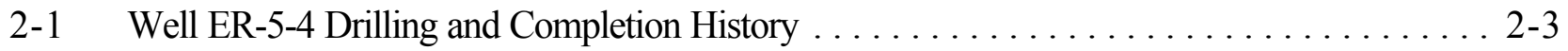

2-2 As-Built Completion Schematic for Well ER-5-4 . . . . . . . . . . . . . . . . . . . . 2-23

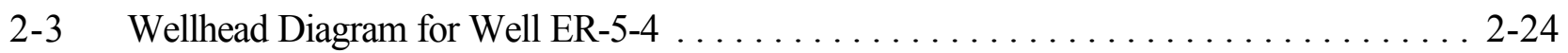

2-4 Planned versus Actual Construction Progress for Well ER-5-4 . . . . . . . . . . . 2 2-30

2-5 Planned versus Actual Cost for Constructing Well ER-5-4 . . . . . . . . . . . . . . 2-31

3-1 Well ER-5-4\#2 Drilling and Completion History $\ldots \ldots \ldots \ldots \ldots \ldots \ldots \ldots \ldots \ldots \ldots \ldots . \ldots \ldots$

3-2 As-Built Completion Schematic for Well ER-5-4\#2 . . . . . . . . . . . . . . . . . 3-18

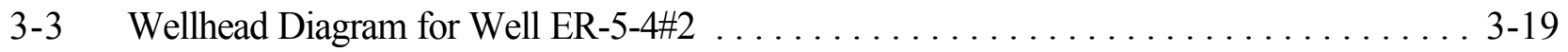

3-4 Planned versus Actual Construction Progress for Well ER-5-4\#2 … . . . . . . . . . 3-23

3-5 Planned versus Actual Cost for Constructing Well ER-5-4\#2 $\ldots \ldots \ldots \ldots \ldots \ldots \ldots . .24$

4-1 Surface Geologic Map of the Well Cluster ER-5-4 Site . . . . . . . . . . . . . . . 4-3

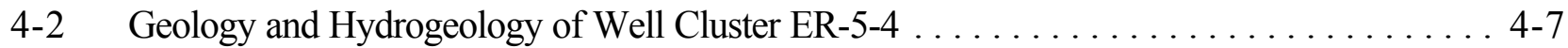

4-3 Predicted and Actual Stratigraphy at Well Cluster ER-5-4 . . . . . . . . . . . . 4-13

4-4 West-East Seismic Profile through Well ER-5-4\#2 . . . . . . . . . . . . . . . . 4-15

4-5 Geologic Cross Section A - A' through Well Cluster ER-5-4 . . . . . . . . . . . . . 4-17

4-6 Hydrogeologic Cross Section B - B' through Cluster Well ER-5-4 . . . . . . . . . . . . 4-19 


\section{List of Tables}

Number

Title

Page

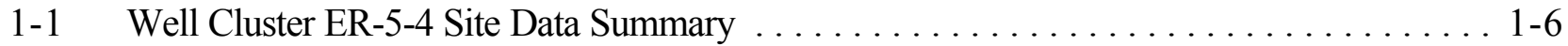

2-1 Abridged Drill Hole Statistics for Well ER-5-4 . . . . . . . . . . . . . . . . . . . 2-2

2-2 Percussion Gun Sidewall Core Samples from Well ER-5-4 . . . . . . . . . . . . 2-12

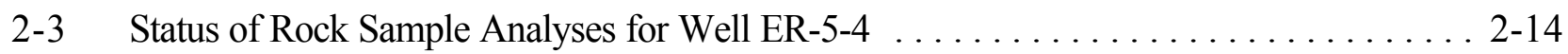

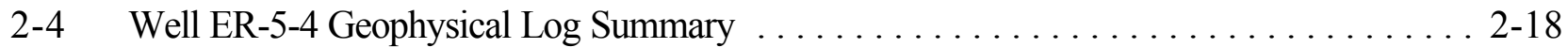

2-5 Completion String Construction Summary for Well ER-5-4 . . . . . . . . . . . . . . . 2-25

3-1 Abridged Drill Hole Statistics for Well ER-5-4\#2 … . . . . . . . . . . . . . . . 3-2

3-2 Percussion Gun Sidewall Core Samples from Well ER-5-4\#2 $\ldots \ldots \ldots \ldots \ldots \ldots \ldots$. $3-12$

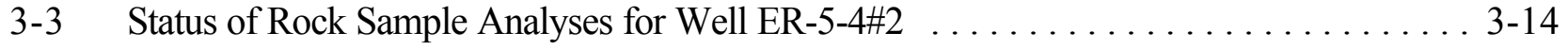

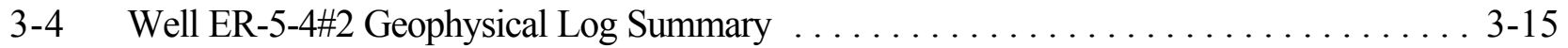

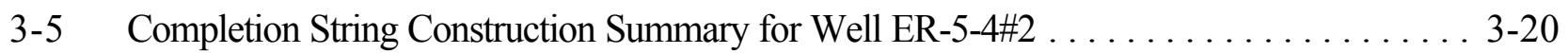

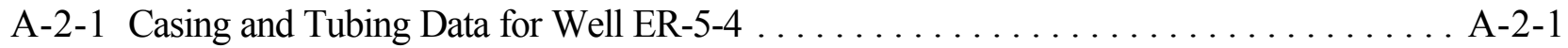

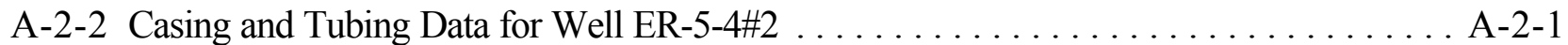

A-3-1 Well ER-5-4 Drilling Fluids $\ldots \ldots \ldots \ldots \ldots \ldots \ldots \ldots \ldots \ldots \ldots \ldots \ldots \ldots \ldots \ldots . \ldots \ldots$

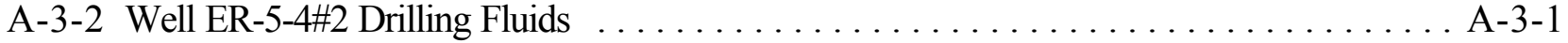

A-3-3 Well ER-5-4 Cement Composition ........................... A-3-2

A-3-4 Well ER-5-4\#2 Cement Composition ........................... A-3-2

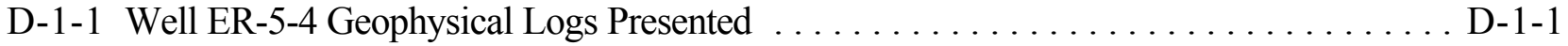

D-2-1 Well ER-5-4\#2 Geophysical Logs Presented ........................ D-2-1 


\section{List of Acronyms and Abbreviations}

\begin{tabular}{|c|c|}
\hline $3-\mathrm{D}$ & three-dimensional \\
\hline BHA & bottom-hole assembly \\
\hline $\mathrm{BN}$ & Bechtel Nevada \\
\hline $\mathrm{C}$ & Celsius \\
\hline $\mathrm{cc}$ & cubic centimeters \\
\hline $\mathrm{cm}$ & centimeter(s) \\
\hline DOE & U.S. Department of Energy \\
\hline DOE/NV & U.S. Department of Energy, Nevada Operations Office \\
\hline DRI & Desert Research Institute \\
\hline EC & Electrical conductivity \\
\hline $\mathrm{F}$ & Fahrenheit \\
\hline FMP & Fluid Management Plan \\
\hline $\mathrm{ft}$ & foot (feet) \\
\hline gal & gallon(s) \\
\hline GBEC & Great Basin Exploration Consultants, Inc. \\
\hline gpm & gallons per minute \\
\hline in. & inch(es) \\
\hline IT & IT Corporation \\
\hline $\mathrm{km}$ & kilometer(s) \\
\hline LANL & Los Alamos National Laboratory \\
\hline LCM & lost circulation material \\
\hline $\mathrm{LiBr}$ & lithium bromide \\
\hline lpm & liters per minute \\
\hline $\mathrm{m}$ & meter(s) \\
\hline Ma & million years ago \\
\hline $\mathrm{mi}$ & mile(s) \\
\hline NAD & North American Datum \\
\hline NNSA/NSO & National Nuclear Security Administration Nevada Site Office \\
\hline NTS & Nevada Test Site \\
\hline $\mathrm{pCi} / \mathrm{L}$ & picoCuries per liter \\
\hline RWMS & Radioactive Waste Management Site \\
\hline TD & total depth \\
\hline TFM & Thermal Flow Meter \\
\hline TWG & Technical Working Group \\
\hline UGTA & Underground Test Area \\
\hline UDI & United Drilling, Inc. \\
\hline USGS & United States Geological Survey \\
\hline
\end{tabular}


This page intentionally left blank. 


\subsection{Introduction}

\subsection{Project Description}

Well Cluster ER-5-4 was drilled for the U.S. Department of Energy (DOE), National Nuclear Security Administration Nevada Site Office (NNSA/NSO; formerly Nevada Operations Office, DOE/NV) in support of the Nevada Environmental Restoration Project at the Nevada Test Site (NTS), Nye County, Nevada. These two wells were constructed as part of the hydrogeologic investigation well program for Frenchman Flat. This program is part of the NNSA/NSO Environmental Restoration Division's Underground Test Area (UGTA) project at the NTS. The goals of the UGTA project include evaluating the nature and extent of contamination in groundwater due to underground nuclear testing, and establishing a long-term groundwater monitoring network. As part of the UGTA project, scientists are developing computer models to predict groundwater flow and contaminant migration within and near the NTS. To build and test these models, it is necessary to collect geologic, geophysical, and hydrologic data from new and existing wells to define groundwater quality, migration pathways, and migration rates.

The goal of constructing, sampling, and hydrologic testing at Well Cluster ER-5-4 is to collect subsurface geologic and hydrologic data in a poorly characterized area near a group of underground nuclear test locations in Frenchman Flat. Data from these wells will allow for more accurate modeling of groundwater flow and radionuclide migration in the region. One of the wells may also function as a long-term monitoring well.

Well Cluster ER-5-4 is located in central Area 5 of the NTS (Figure 1-1), approximately 1.6 kilometers $(\mathrm{km})$ (1.0 miles [mi]) northwest of the Frenchman Lake playa. The cluster consists of two boreholes drilled 30.5 meters $(\mathrm{m})$ (100 feet [ $\mathrm{ft}]$ ) apart on the same drill pad (Figure 1-2). The elevation of the dirt-fill drill pad at the wellheads is $954.5 \mathrm{~m}(3,131.7 \mathrm{ft})$ above mean sea level. The Nevada State plane coordinates and elevation at both wellheads are listed in Table 1-1, along with additional site summary and survey information.

IT Corporation (IT) was the principal environmental contractor for the project, and IT personnel collected geologic and hydrologic data during drilling. The drilling company was United Drilling, Incorporated (UDI), a subcontractor to Bechtel Nevada (BN). Site supervision, engineering, construction, inspection, and geologic support were provided by BN. The roles and responsibilities 
This page intentionally left blank. 


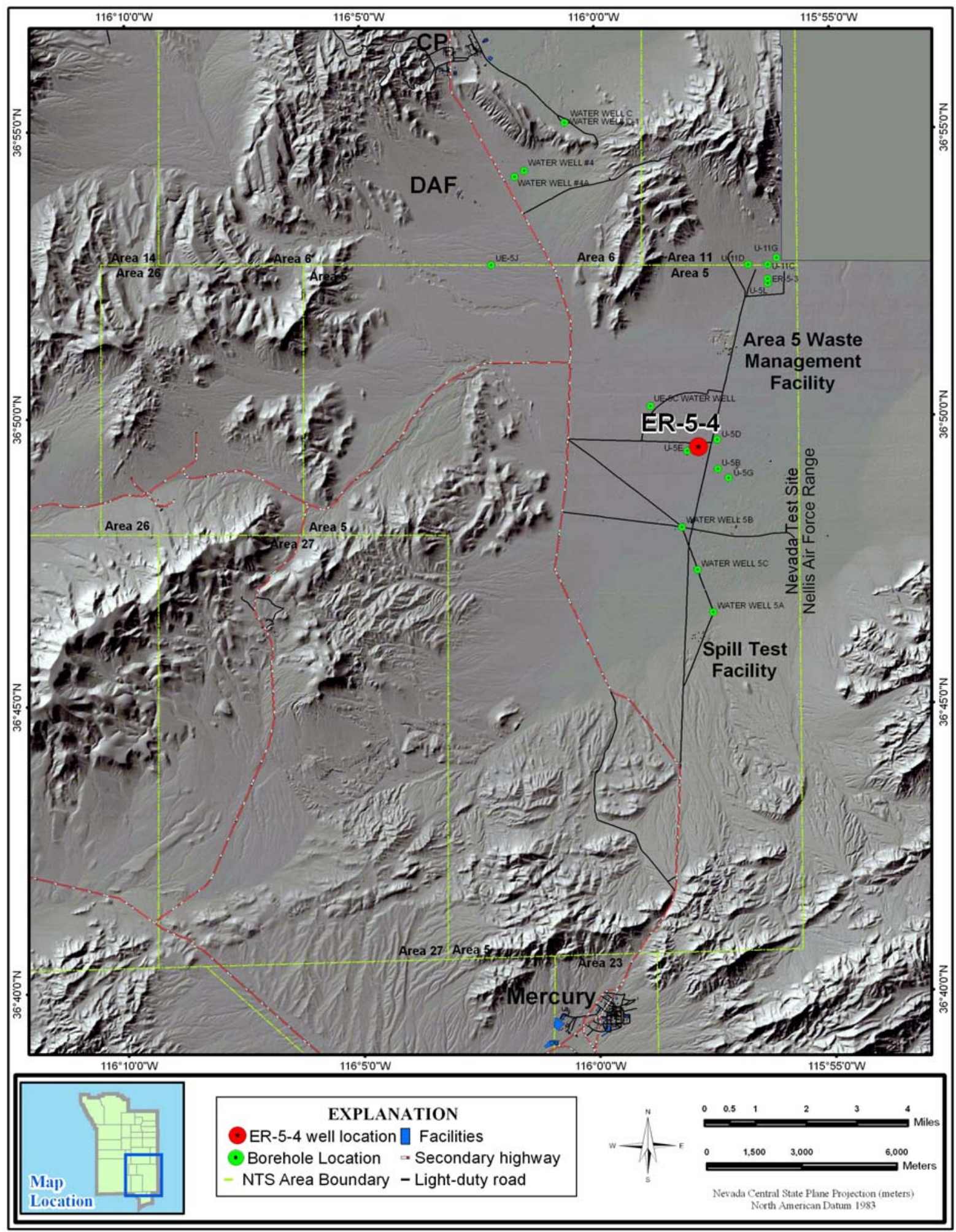

Figure 1-1

Reference Map Showing Location of Well Cluster ER-5-4 
This page intentionally left blank. 


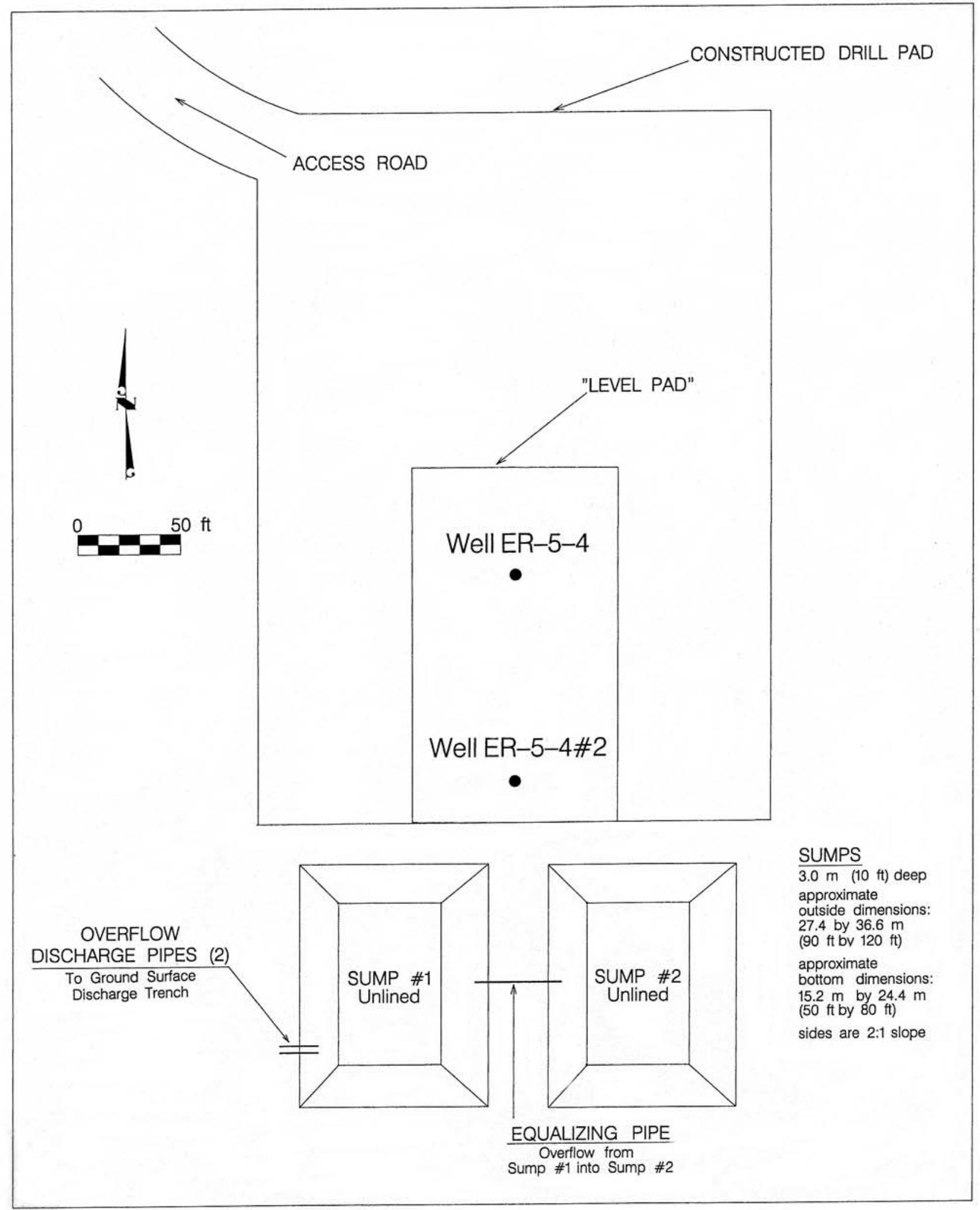

Figure 1-2

Drill Site Configuration for Well Cluster ER-5-4 
Table 1-1

Well Cluster ER-5-4 Site Data Summary

\begin{tabular}{|c|c|c|c|}
\hline \multicolumn{2}{|c|}{$\begin{array}{l}\text { Well Designation } \\
\text { (Date TD reached) }\end{array}$} & $\begin{array}{c}\text { ER-5-4 } \\
\text { (March 18, 2001) }\end{array}$ & $\begin{array}{c}\text { ER-5-4\#2 } \\
\text { (September 11, 2002) }\end{array}$ \\
\hline \multirow{4}{*}{$\begin{array}{c}\text { Site } \\
\text { Coordinates }^{a}\end{array}$} & $\begin{array}{l}\text { Nevada State Plane (central } \\
\text { zone) (NAD 83) meters }\end{array}$ & $\begin{array}{l}\text { N } 6,230,353.7 \\
\text { E } 562,655.5\end{array}$ & $\begin{array}{l}\text { N } 6,230,323.2 \\
\text { E } 562,655.4\end{array}$ \\
\hline & $\begin{array}{l}\text { Nevada State Plane (central } \\
\text { zone) (NAD 83) feet }\end{array}$ & $\begin{array}{l}\text { N 20,440,752.2 } \\
\text { E } 1,845,978.9\end{array}$ & $\begin{array}{l}\text { N 20,440,652.0 } \\
\text { E 1,845,978.6 }\end{array}$ \\
\hline & $\begin{array}{l}\text { Nevada State Plane (central } \\
\text { zone) (NAD 27) feet }\end{array}$ & $\begin{array}{l}N 755,751.3 \\
\text { E } 705,819.9\end{array}$ & $\begin{array}{l}\text { N } 755,651.2 \\
E 705,819.6\end{array}$ \\
\hline & $\begin{array}{c}\text { Universal Transverse } \\
\text { Mercator (Zone 11) (NAD 83) } \\
\text { meters }\end{array}$ & $\begin{array}{l}\text { N } 4,075,874.5 \\
\text { E } 592,364.5\end{array}$ & $\begin{array}{l}\text { N } 4,075,844.0 \\
\text { E } 592,364.5\end{array}$ \\
\hline \multicolumn{2}{|c|}{ Surface Elevation ${ }^{b}$} & $954.5 \mathrm{~m}(3,131.7 \mathrm{ft})$ & $954.5 \mathrm{~m}(3,131.7 \mathrm{ft})$ \\
\hline \multicolumn{2}{|r|}{ Drilled Depth } & $1,137.5 \mathrm{~m}(3,732 \mathrm{ft})$ & $2,133.6 \mathrm{~m}(7,000 \mathrm{ft})$ \\
\hline \multicolumn{2}{|c|}{ Preliminary Fluid-Level Depth ${ }^{c}$} & $221.3 \mathrm{~m}(726.0 \mathrm{ft})$ & $215.7 \mathrm{~m}(707.6 \mathrm{ft})$ \\
\hline \multicolumn{2}{|c|}{ Fluid-Level Elevation } & $733.6 \mathrm{~m}(2,405.7 \mathrm{ft})$ & $732.8 \mathrm{~m}(2,404.1 \mathrm{ft})$ \\
\hline
\end{tabular}

a Measurement made by BN Survey. 1983 or 1927 North American Datum (NAD).

b Measurement made by BN Survey. Elevation at top of construction pad. 1929 National Geodetic Vertical Datum.

c Well ER-5-4: determined on February 16, 2001, prior to introduction of bentonite mud (IT, 2001b). Well ER-5-4 \#2: determined on September 18, 2002 (IT, 2003).

of these and other contractors involved in the project are described in Contract Number DE-RP-08-95NV11808, and in BN Field Activity Work Plans Number D-002-001.01 and Number D-006-001.02 (BN, 2001, 2002). The UGTA Technical Working Group (TWG), a committee of scientists and engineers comprising NNSA/NSO, Lawrence Livermore National Laboratory, Los Alamos National Laboratory (LANL), and contractor personnel, provided additional technical advice during drilling, design, and construction of the well. See Frenchman Flat Hydrogeologic Investigation Wells Drilling and Completion Criteria (IT, 2000) and an addendum to that document (IT, 2001a) for descriptions of the general plan and goals of the Well Cluster ER-5-4 project, as well as specific goals for both wells.

General guidelines for managing fluids used and generated during drilling, completion, and testing of UGTA wells are provided in the UGTA Fluid Management Plan (FMP) (DOE/NV, 1999), an 
attachment to the UGTA Waste Management Plan (DOE/NV, 1996). Estimates of production of fluid and drill cuttings for these holes are given in Appendix $\mathrm{C}$ of the drilling and completion criteria document for the Frenchman Flat drilling project (IT, 2000), along with sampling requirements and contingency plans for management of any hazardous waste produced. All activities were conducted according to the Nevada Environmental Restoration Project Health and Safety Plan (DOE/NV, 1998), and the UGTA Health and Safety Plan (BN, 2001).

This report presents construction data and summarizes scientific data gathered during drilling and installation of the completion strings in Wells ER-5-4 and ER-5-4\#2. Well data reports prepared by IT (IT, 2001b, 2003) contain additional information on fluid management, waste management, and environmental compliance. Information on well development, aquifer testing, and groundwater analytical sampling will be compiled and disseminated separately.

\subsection{Objectives}

The primary purpose of constructing Well Cluster ER-5-4 was to obtain information that will help characterize the hydrogeology of this part of Frenchman Flat. The primary scientific objectives for these wells, as discussed in the drilling criteria documents (IT, 2000, 2001a), include the following:

- Validate the current hydrogeologic framework model for this part of Frenchman Flat, with special interest in the base of the alluvium and the top of the lower carbonate aquifer.

- Verify the presence of the tuff confining unit and obtain data on its geologic and hydrologic properties.

- Document any differences in the physical nature of the alluvial aquifer with depth.

- Obtain velocity data and geologic control to aid interpretation of the recently conducted 3-dimensional (3-D) seismic survey.

- Obtain geologic samples for detailed mineralogical analyses.

- Obtain water level data to refine knowledge of the local water table.

- Obtain data to determine vertical head distribution/vertical hydraulic gradient at various locations within the alluvial aquifer and between the various hydrogeologic units. 
- Obtain vertical and horizontal conductivity measurements.

- Obtain data to address potential contaminant transport processes such as a hydrologic "short cut" from contaminant sources in the alluvium to the lower carbonate aquifer via a fault. The deeper well in this cluster was expected to intercept a geophysically inferred fault.

- Serve as a multi-level observation well during full-scale aquifer tests.

- Provide long-term monitoring point(s) for the evaluation of temporal changes in water levels and groundwater chemistry.

Some of these objectives will not be met until additional work, outside the scope of this report, is completed, including installing pumps and conducting hydraulic testing, and analyzing geologic and hydrologic data from these wells and other data for the Frenchman Flat area. Objectives for individual wells of the cluster are listed in the well-specific portions of this report.

\subsection{Project Summary}

This section summarizes construction operations at Well Cluster ER-5-4; the details are provided in Sections 2.0 through 8.0.

\subsubsection{Well ER-5-4}

The surface conductor hole was constructed by augering a 121.9-centimeter (cm) (48-inch [in.]) diameter hole to a depth of $37.2 \mathrm{~m}$ (122 ft) and installing a string of 30-in. casing. Drilling of the main hole with a 171/2-in. rotary bit, using an air-water-foam-polymer fluid in conventional circulation, began on February 10, 2001, and continued to the depth of $312.1 \mathrm{~m}(1,024 \mathrm{ft})$. At that depth circulation was lost and drilling was suspended for geophysical logging and installation of the surface casing. The diameter of the hole was increased to $66.0 \mathrm{~cm}$ ( $26 \mathrm{in}$.) by reaming, to allow installation of a string of 20-in. surface casing. The 20-in. casing was set on February 24, 2001, at the depth of $279.1 \mathrm{~m}$ (915.5 ft). Drilling resumed with bentonite mud and a 171/2-in. bit to the depth of $510.5 \mathrm{~m}(1,675 \mathrm{ft})$. At this point, drilling was again suspended for geophysical logging, and then the $13 \mathrm{~d}$-in. intermediate casing string was landed at $510.0 \mathrm{~m}(1,673.3 \mathrm{ft})$. Drilling continued with a 121/4-in. bit to a total depth (TD) of 1,137.5 m (3,732 ft), which was reached on March 18, 2001. Several attempts at geophysical logging made during the week after TD was reached were hampered by blockages due to accumulations of fill in the borehole. The entire drilling operation was hampered by borehole sloughing and loss of drill fluid to the formation. Efforts to overcome these problems included the use of bentonite in the drill fluid, the use of lost-circulation material, and cementing and redrilling parts of the hole. 
Water production was first noted at the depth of approximately $223.1 \mathrm{~m}(732 \mathrm{ft})$, and reached a maximum of approximately 2,271 liters per minute (lpm) (600 gallons per minute (gpm) at the depth of about $310 \mathrm{~m}$ (2,000 ft). A preliminary composite fluid-level tag was made at the depth of $221.3 \mathrm{~m}$ (726 ft). Radionuclide levels above background were encountered in only two short intervals, and the maximum tritium activity of 5,028 picoCuries per liter ( $\mathrm{pCi} / \mathrm{L}$ ) was measured in a sample of drill fluid from the depth of $298.7 \mathrm{~m}$ (980 ft) (IT, 2001b).

Composite drill cuttings were collected every $3 \mathrm{~m}$ (10 ft) from $36.6 \mathrm{~m}$ (120 ft) to TD, and 154 sidewall core samples were taken at various depths below $192.0 \mathrm{~m}(630 \mathrm{ft})$. Open-hole geophysical logging of the well was conducted to help verify the geology and characterize the hydrology of the rocks; some logs also aided in the construction of the well by indicating borehole volume and condition, and cement location. The well penetrated Quaternary and Tertiary alluvium from the surface to $1,118.6 \mathrm{~m}$ $(3,670 \mathrm{ft})$ and Tertiary volcanic rocks from 1,118.6 $\mathrm{m}(3,670 \mathrm{ft})$ to TD. No recognizable faults were encountered.

The as-built completion design for Well ER-5-4 provides access to the alluvial aquifer at 3 depths. A bull-nosed piezometer string of $2 f$-in. stainless-steel tubing is set in the annulus of the 20 -in. casing at a depth of $247.8 \mathrm{~m}(812.8 \mathrm{ft})$. The piezometer string is slotted in the depth interval 220.2 to $247.4 \mathrm{~m}$ (722.6 to $811.7 \mathrm{ft}$ ). A string of 51/2-in. stainless-steel casing was landed at the depth of $1,048.0 \mathrm{~m}$ $(3,438.3 \mathrm{ft})$. The bull-nosed string has 2 slotted intervals, at 955.9 to $1,021.1 \mathrm{~m}(3,136.3$ to $3,350.1 \mathrm{ft})$ and at 539.5 to $644.2 \mathrm{~m}(1,770.0$ to $2,113.4 \mathrm{ft})$. The $5 \frac{1}{2} 2$-in. casing hangs from a string of 7e -in. carbon-steel production casing that extends to the ground surface. The completion string was gravel-packed across the slotted intervals and the remaining annular space was filled with gravel, sand, and cement to the depth of $499.9 \mathrm{~m}$ (1,640 ft) on March 31, 2001. No pump was installed at the time of completion, but will be inserted as needed for hydrologic testing and sampling activities.

\subsubsection{Well ER-5-4\#2}

The surface conductor hole for Well ER-5-4\#2 was constructed immediately after that for Well ER-5-4 by augering a 121.9-cm (48-in.) diameter hole to a depth of $35.1 \mathrm{~m}(115 \mathrm{ft})$ and installing a string of 30 -in. casing. Drilling of the main hole with a 171/2-in. rotary bit, using an air-water-foam-polymer fluid in conventional circulation, began 17 months later, on July 15, 2002. 
Based on experience during drilling of Well ER-5-4, measures were taken to try to minimize borehole instability. However, lost circulation and borehole sloughing were ongoing problems during drilling to the depth of $371.9 \mathrm{~m}(1,220 \mathrm{ft})$. At that depth, on July 22, 2002, a crack opened at the surface, with drilling fluid flowing out of it, and extending for a distance of 15.2 to $21.3 \mathrm{~m}$ (50 to $70 \mathrm{ft}$ ) from the well collar on two sides of the hole. The crack was observed via down-hole camera, to extend across the borehole from the base of the conductor casing at $33.8 \mathrm{~m}(111 \mathrm{ft})$ to a depth of $71.6 \mathrm{~m}(235 \mathrm{ft})$. To seal off the crack and to control sloughing of the borehole, the hole was opened to $66.0 \mathrm{~cm}$ (26 in.) in diameter to accommodate a string of 20 -in. surface casing.

The 20 -in. casing was run in the hole to the top of fill at $266.5 \mathrm{~m}(874.5 \mathrm{ft})$, and the bottom was cemented. Borehole sloughing and lost circulation persisted, and the bottom of the surface hole was cemented and re-drilled 4 times, before drilling resumed at the original depth of the $44.5-\mathrm{cm}\left(17 \frac{1}{2}-\mathrm{in}\right.$.) surface hole at $371.9 \mathrm{~m}(1,220 \mathrm{ft})$ on August 8, 2002. Additional problems with borehole instability were again encountered below the depth of $923.5 \mathrm{~m}(3,030 \mathrm{ft})$. The $13 \mathrm{~d}-\mathrm{in}$. intermediate casing was set at the depth of $965.9 \mathrm{~m}(3,169.0 \mathrm{ft})$ on August 16, 2002, after the depth of 1,052.5 $\mathrm{m}(3,453 \mathrm{ft})$ was reached. The hole was advanced with a 121/4-in. bit to $1,573.7 \mathrm{~m}(5,163 \mathrm{ft})$ on August 24, 2002, despite continued borehole instability. Geophysical logging was conducted, and then a string of $9 e$-in. casing was set at the depth of $1,477.9 \mathrm{~m}(4,848.8 \mathrm{ft})$. Drilling resumed with a $83 / 4$-in. bit with few problems to the TD of 2,133.6 m (7,000 ft), reached on September 11, 2002.

The drillers repeatedly encountered severe sloughing of the borehole, large accumulations of fill, and loss of large quantities of drill fluid to the formation, despite efforts to overcome these problems, which included the use of bentonite in the drill fluid, the use of lost-circulation material, and cementing and redrilling parts of the hole.

Water production was first noted at the depth of approximately $221.6 \mathrm{~m}(727 \mathrm{ft})$, and reached a maximum of approximately $2,157 \mathrm{lpm}(570 \mathrm{gpm})$ at the depth of about $1,996 \mathrm{~m}(6,550 \mathrm{ft})$. A preliminary composite fluid level was measured soon after completion at the depth of $215.7 \mathrm{~m}$ (707.6 ft). Radionuclide levels above background were encountered in two intervals, and the maximum tritium activity of $1,792 \mathrm{pCi} / \mathrm{L}$ was measured in a sample of drill fluid from the depth of 1,576.1 m $(5,171 \mathrm{ft})$. 
Composite drill cuttings were collected every $3 \mathrm{~m}(10 \mathrm{ft})$ from $35.1 \mathrm{~m}(115 \mathrm{ft})$ to TD, and 2 partial sidewall core samples were obtained. Open-hole geophysical logging of the well was conducted to help verify the geology and characterize the hydrology of the rocks; some logs also aided in the construction of the well by indicating borehole volume and condition, and cement location. The borehole penetrated Quaternary and Tertiary alluvium from the surface to the depth of 1,120.4 m $(3,676 \mathrm{ft})$, and Tertiary volcanic rocks from that depth to the TD. Paleozoic sedimentary rocks were not encountered in Well ER-5-4\#2. No recognizable faults were encountered.

A single completion zone was constructed within Tertiary volcanic rocks. The completion string consists of $169.7 \mathrm{~m}(556.9 \mathrm{ft})$ of 51/2-in. stainless-steel casing, hung from a liner hanger set at 1,437.1 m $(4,715.0 \mathrm{ft})$ within the $9 \mathrm{e}$-in. carbon-steel intermediate casing. The bottom of the $5 \frac{1}{2} 2$-in. casing terminates in a bull-nose at the depth of 2,030.0 m (6,660.0 ft). The casing is slotted in the interval $1,976.9$ to $2,029.3 \mathrm{~m}(6,486.0$ to $6,657.7 \mathrm{ft})$. No gravel-pack or cement were utilized in the completion. The slotted interval coincides with a fractured interval that produced water during drilling.

\title{
1.4 Project Manager
}

Inquiries concerning Well Cluster ER-5-4 should be directed to the UGTA Project Manager at:

\author{
U.S. Department of Energy \\ National Nuclear Security Administration \\ Nevada Site Office \\ Environmental Restoration Division \\ Post Office Box 98518 \\ Las Vegas, Nevada 89193-8518
}


This page intentionally left blank. 


\subsection{Well ER-5-4}

This section contains detailed descriptions of the drilling process and fluid management issues, geologic data collection, and completion information for Well ER-5-4. Subsequent hydrologic sampling and testing are beyond the scope of this report.

\section{$2.1 \quad$ Well-Specific Objectives}

The scientific objectives for Well ER-5-4 include those listed for the well cluster in Section 1.2. However, the specific goal of this first well in the cluster was to penetrate the alluvial section and install completions within 3 zones of interest in the saturated alluvium. Well ER-5-4 was planned to reach TD in the top of the underlying volcanic rocks.

\subsection{Drilling Summary}

This section contains detailed descriptions of the drilling process and fluid management issues.

\subsubsection{Introduction}

The general drilling requirements for Well ER-5-4 were provided in Frenchman Flat Hydrogeologic Investigation Wells Drilling and Completion Criteria (IT, 2000) and an addendum to that document (IT, 2001a). Specific requirements for Well ER-5-4 were outlined in Field Activity Work Plan Number D-002-001.01 (BN, 2001). A summary of drilling statistics for the well is given in Table 2-1. Figure 2-1 is a chart of the drilling and completion history for Well ER-5-4. The following information was compiled primarily from $\mathrm{BN}$ daily drilling reports.

\subsubsection{Drilling History}

Field operations at Well ER-5-4 began on January 24, 2001, when a BN crew began dry-augering a 121.9-cm (48-in.) diameter conductor hole. The conductor hole was completed the next day to a depth of $37.2 \mathrm{~m}(122 \mathrm{ft})$, and a string of 30-in. conductor casing was set at $36.3 \mathrm{~m}(119 \mathrm{ft})$. The bottom of the casing was cemented into place with neat type II cement, and the annulus was cemented to ground level. A short hiatus in the construction of Well ER-5-4 followed, during which the BN crew augered the "rat hole," "mouse hole," and anchor holes for Well ER-5-4, and drilled the conductor hole for the nearby planned Well ER-5-4\#2.

The UDI crew rigged up the Wilson Mogul 42B rig from on February 7 to 10, 2001, and tagged cement at the depth of $34.7 \mathrm{~m}(114 \mathrm{ft})$. Drilling resumed with a center-punch assembly consisting of a 


\section{Table 2-1}

\section{Abridged Drill Hole Statistics for Well ER-5-4}

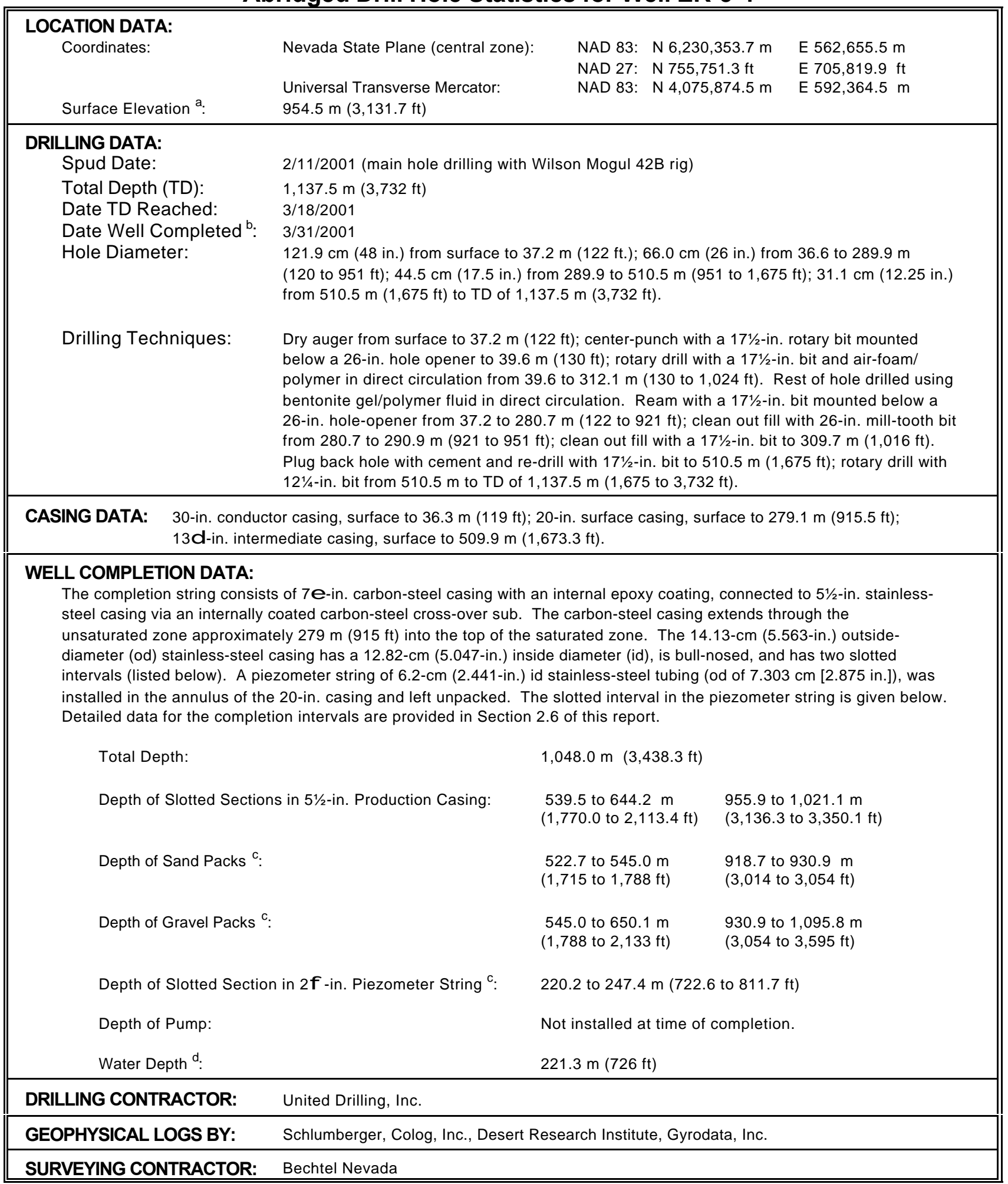

a Elevation of ground level at wellhead. 1929 National Geodetic Vertical Datum.

b Date completion string was cemented. Pump will be installed as needed for pumping or sampling.

c Gravel and sand adjacent to slotted intervals of 51/2-inch casing only. Includes fill at 930.9 to $933.9 \mathrm{~m}$ (3,054 to $3,064 \mathrm{ft}$ ). Additional gravel layers were used as stemming outside blank casing sections. Piezometer string was not gravel-packed. See Table 2-5.

d Determined from gamma ray log run on February 16, 2001, prior to introduction of bentonite mud in the borehole (IT, 2001b). 


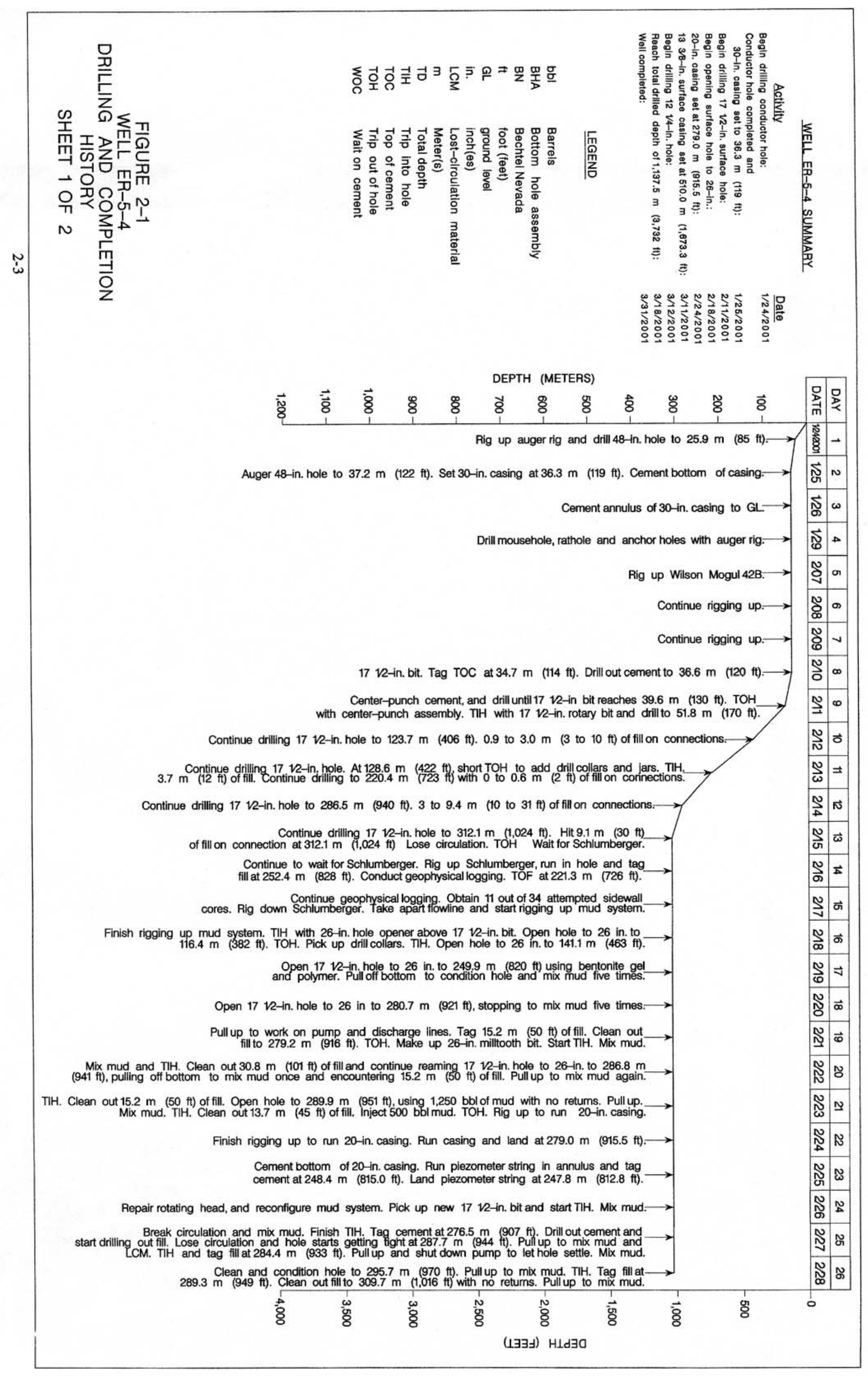




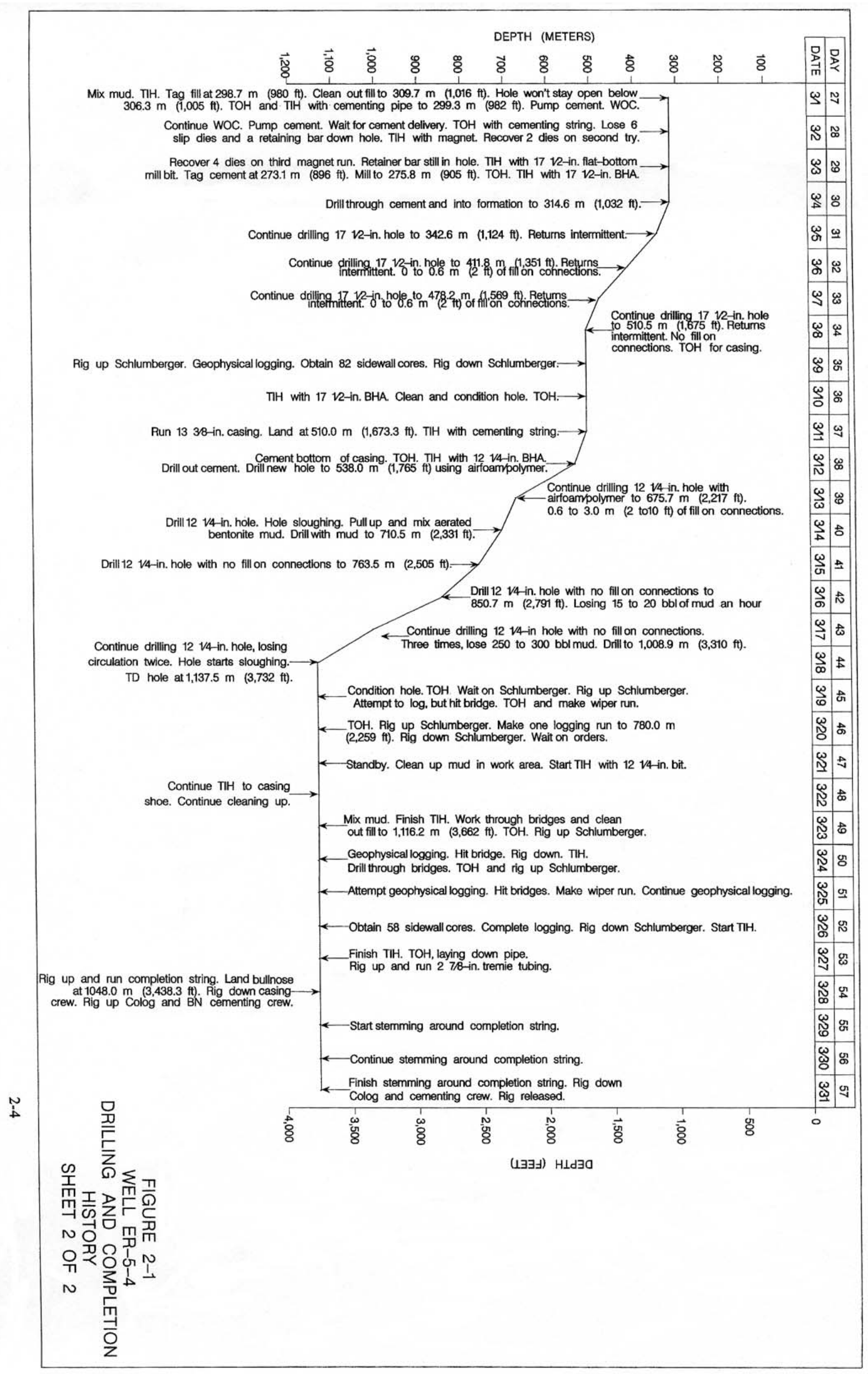


171/2-in. rotary bit mounted below a 26 -in. hole opener. When the hole opener had cleared the bottom of

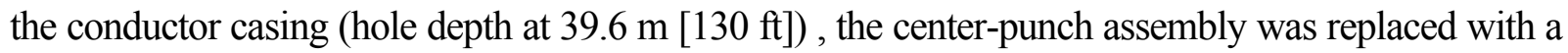
171/2-in. bottom-hole assembly (BHA), and drilling continued. The drilling fluid was a mix of air, water, and foam ("air-foam") in conventional circulation, with a polymer additive. As the hole deepened, as much as $3.7 \mathrm{~m}$ (12 ft) of fill material, sloughed from the borehole wall, was encountered whenever drilling was stopped to add drill pipe ("make a connection"). Below the static water level at approximately $221.3 \mathrm{~m}$ (726 ft), sloughing increased dramatically, with 3.0 to $9.4 \mathrm{~m}$ (10 to $31 \mathrm{ft}$ ) of fill encountered on each connection. In an effort to minimize sloughing and maintain circulation, additional foam and polymer were added to the drill-fluid mix, and another compressor was added. Drilling of the 44.5-cm (171/2-in.) hole continued to a depth of $312.1 \mathrm{~m}$ (1,024 ft) on February 15, 2001, when circulation was lost. At that point drilling was stopped for geophysical logging, which was conducted February 16 to 17, 2001. Fill was tagged by loggers at $252.4 \mathrm{~m}(828 \mathrm{ft})$. The decision was made to install the surface casing to protect the upper part of the hole from additional erosion, and the NNSA/NSO project manager approved the use of bentonite mud (a heavier drill fluid) in an effort to stabilize the borehole.

After the new system for circulating the heavier mud was set up, drillers put together a BHA with a 26-in. hole-opener mounted above a 171/2-in. rotary bit, and the next 4 days were spent cleaning out fill and opening the hole to allow installation of the 20 -in. surface casing. Bentonite mud was used for reaming below the depth of $141.1 \mathrm{~m}$ (463 ft). Progress in opening the hole was slow due to difficulty in mixing enough mud to keep up with fluid loss to the formation. On February 21, 2001, when the hole had been opened to a depth of $280.7 \mathrm{~m}$ (921 ft), the BHA was replaced with a 26-in. mill-tooth bit. Reaming of the hole continued to $289.9 \mathrm{~m}$ (951 ft) with considerable difficulty due to sloughing of the borehole and the need to mix large amounts of bentonite mud to keep up with fluid loss.

A casing subcontractor set a string of 20 -in. casing at $279.1 \mathrm{~m}$ (915.5 ft) on February 24, 2001. To cement the bottom of the casing, drill pipe was stabbed into the casing shoe, the seal was checked, and neat type II cement was pumped down the drill pipe. Water was then pumped down the pipe to displace the cement into the casing annulus. The top of cement in the annulus was later tagged at the depth of approximately $248.4 \mathrm{~m}(815 \mathrm{ft})$. No baskets were installed on the 20 -in. casing, and the upper part of the annulus remains uncemented. A string of slotted $2 f$-in. tubing was installed adjacent to the borehole wall in the annulus of the 20-in. casing, and landed at $247.8 \mathrm{~m}(812.8 \mathrm{ft}$ ), to serve as a piezometer (see Section 2.6). 
The mud circulation system was modified to route cuttings to a shaker tray to aid in removing the thick bentonite mud from the cuttings. Drilling resumed on February 27, 2001, with bentonite mud and a new $17 \frac{1}{2}$-in. bit. Cement was drilled from inside the bottom of the casing from 276.5 to $279.2 \mathrm{~m}$ (907 to $916 \mathrm{ft}$ ), and drilling continued into fill. Progress was slowed by borehole sloughing and loss of circulation while drilling out fill. No fluid was returned during drilling from 283.5 to $309.7 \mathrm{~m}$ (930 to $1,016 \mathrm{ft}$ ). Lostcirculation material (LCM) consisting of cedar fiber and a commercial cellulose-based sealant was mixed into the mud to try to seal the borehole walls, but after reaching the depth of $309.7 \mathrm{~m}(1,016 \mathrm{ft})$ the drillers could not keep the borehole open. The decision was made to cement (or "plug back") and then re-drill the lower portion of the hole, so on March 1, 2001, the BHA was removed and open-ended drill pipe was installed to serve as a cementing string. The hole was cemented in 2 stages, with 16.1 cubic meters (570 cubic feet) of neat type II cement emplaced on the top of fill tagged at the depth of $308.5 \mathrm{~m}$ $(1,012 \mathrm{ft})$. While the cement string was being tripped out, 6 slip dies and a retaining bar were lost in the hole. After 3 runs with a magnet, all 6 dies were recovered, but the retaining bar remained in the hole, so a $17 \frac{1}{2}$-in. flat-bottom mill bit was lowered into the hole, tagging the top of cement at $273.1 \mathrm{~m}$ (896 ft). After the retaining bar and cement were milled out to $275.8 \mathrm{~m}(905 \mathrm{ft})$, the mill bit was replaced with the standard 171/2-in. BHA.

Drilling resumed through the cement plug, but alluvium found mixed with the cuttings indicates that the hole may have partially sidetracked the cement at $295.0 \mathrm{~m}$ (968 ft). On March 4, 2001, drilling reached the original hole depth of $312.1 \mathrm{~m}(1,024 \mathrm{ft})$, and over the next 4 days the hole was advanced with a 171/2-in. bit to $510.5 \mathrm{~m}(1,675 \mathrm{ft})$, with little or no fill encountered on connections. A compressor was used to aerate drilling mud, and LCM was added to the mud, but returns of cuttings and mud were intermittent due to frequent loss of fluid to the formation.

The decision was made to stop drilling and set the intermediate casing at $510.5 \mathrm{~m}(1,675 \mathrm{ft})$, so drillers pulled the drill string up, waited for 30 minutes, and tripped back in to check for fill. None was found, so the drill string was removed and geophysical logging and sidewall sampling were conducted on March 9, 2001 (sidewall cores taken at the depths 290.8 and $296.9 \mathrm{~m}$ [954 and $972 \mathrm{ft}$ ] recovered only cement). After drillers made a "wiper run," the casing subcontractor landed a string of $13 \mathrm{~d}$-in. casing at the depth of $510.0 \mathrm{~m}(1,673.3 \mathrm{ft})$. Drill pipe was stabbed into the casing shoe, the seal was checked, and neat type II cement was pumped down the drill pipe to seal the bottom of the casing. The top of the cement in the annulus of the $13 \mathrm{~d}$-in. casing was later estimated with the aid of geophysical logs to be at $442.6 \mathrm{~m}(1,452 \mathrm{ft})$. No cement baskets were installed on the $13 \mathrm{~d}$-in. casing, and the top of the casing remains uncemented. 
Drilling resumed on March 12, 2001, with a 121/4-in. bit and air-foam with polymer, but problems with borehole sloughing necessitated a return to the use of aerated bentonite mud below the depth of $694.0 \mathrm{~m}$ $(2,277 \mathrm{ft})$. Drilling continued with intermittent loss of circulation, but little or no fill on connections. Drillers encountered increasing amounts of fill on connections below the depth of 1,115.6 $\mathrm{m}(3,660 \mathrm{ft})$, until reaching the TD of 1,137.5 $\mathrm{m}(3,732 \mathrm{ft})$ on March 18, 2001.

Immediately after reaching TD, the drillers circulated fluid for $3 \frac{1}{2}$ hours to clean and condition the hole. The drill string was pulled up, and after 30 minutes tripped back in to check for fill. An accumulation of

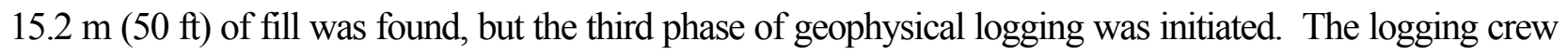
could not work their tool past a bridge of fill material at $651.4 \mathrm{~m}(2,137 \mathrm{ft})$, so they rigged down and the drillers made a wiper run with the 12/4-in. bit. On the second logging attempt, it was possible to log to the depth of $780.0 \mathrm{~m}(2,559 \mathrm{ft})$, but at this time work was suspended because the amount of mud on the drill pad was causing a hazardous situation.

Crews spent the next 38 hours standing by while a plan was made to address the situation and cleaning up mud from the area during daylight hours. On March 23, 2001, drillers resumed reaming and cleaning out the hole in preparation for another attempt at geophysical logging. When logging was attempted the next day, loggers tagged fill at 1,100.0 m (3,609 ft), and successfully made one logging run; however, attempts to obtain other $\operatorname{logs}$ that day failed due to an obstruction in the hole at $553.5 \mathrm{~m}(1,816 \mathrm{ft})$. Once again, drillers cleaned out the hole, washing, reaming, and drilling through bridges. After more attempts at geophysical logging failed the next day, drillers made another wiper run. Loggers tagged fill at 1,095.8 $\mathrm{m}$ (3,595 ft) and successfully completed logging March 26, 2001. Installation of the completion string began on March 27, 2001. Demobilization from the Well ER-5-4 site began after gravel-packing and cementing were completed on March 31, 2001.

The directional survey run in the well on May 10, 2001, indicates that at the lowest surveyed depth of $951.6 \mathrm{~m}(3,122 \mathrm{ft})$ the hole had drifted $9.8 \mathrm{~m}(32 \mathrm{ft})$ to the southwest of the collar location, and that the hole is relatively straight (no "dog-legs").

A graphical depiction of drilling parameters including penetration rate, revolutions per minute, pump pressure, and weight on the bit is presented in Appendix A-1. See Appendix A-2 for a listing of tubing and casing materials. Drilling fluids and cements used in Well ER-5-4 are listed in Appendix A-3. 


\subsubsection{Drilling Problems}

Significant drilling problems associated with borehole instability were encountered at Well ER-5-4.

Sloughing of material from the borehole wall produced large "washouts" and frequently produced up to $15.2 \mathrm{~m}$ (50 ft) of fill on connections, especially below the static water level. Loss of fluid circulation was also a problem. Significant amounts of drilling fluid were lost to the formation, and drilling progress was frequently slowed for mixing additional fluid. It was necessary to use bentonite mud and LCM in an effort to stabilize the borehole and maintain circulation. Because of poor drilling conditions, the interval from 279.2 to $308.5 \mathrm{~m}$ ( 916 to $1,012 \mathrm{ft}$ ) was cemented and re-drilled. However, similar difficulties persisted to the termination of drilling. Drilling continued $70.7 \mathrm{~m}(232 \mathrm{ft})$ below the originally planned depth of $1,066.8 \mathrm{~m}(3,500 \mathrm{ft})$ in an effort to tag the top of the volcanic rocks which were encountered at approximately $1,118.6 \mathrm{~m}(3,670 \mathrm{ft})$.

\subsubsection{Fluid Management}

This section provides a summary of fluid management activities during drilling operations at Well ER-5-4. Much of the information presented is from IT (2001b) where additional fluid management information is available. Fluids and drill cuttings produced during drilling operations at Well ER-5-4 were managed according to the methods prescribed in the UGTA FMP (DOE/NV, 1999) and associated state-approved waivers (Liebendorfer, 2000).

To manage the anticipated water production, 2 sumps (infiltration basins) were constructed prior to drilling (Figure 1-2). Each sump has a capacity of 2,029.6 cubic meters (71,675 cubic feet) or 536,322 gallons at a 3.0-m (10-ft) fluid level. No contaminants were expected during drilling at this site, so neither sump was lined prior to drilling. Two overflow pipes were installed in the western-most sump (Sump \#1), but no overflow pipes were installed in Sump \#2.

The drill fluid was circulated down the inside of the drill string and back up the hole through the annulus (conventional or direct circulation) and then discharged into a sump. Return fluids were piped to Sump \#1 until bentonite mud was added to the fluid mix; returns were then diverted to Sump \#2. Overflow fluids from Sump \#1 were discharged to a trench that connects to the "Cambric ditch" which ultimately conveys fluids to the Frenchman Flat playa. When the shaker tray was installed for removal of mud from the cuttings, a trench was constructed to allow the excess mud to flow to Sump \#2.

Water used to prepare drilling fluids came from fill stands located at the Radioactive Waste Management Site (RWMS) in Area 5 and in Mercury, in Area 23. Water from various NTS water wells on the site 
water well system feeds these fill stands; the stands typically may contain a mix of waters from Water Well 4, Water Well 4a, or the Army Well. Lithium bromide ( $\mathrm{LiBr}$ ) was added to the drill fluid as a tracer to provide a means of estimating groundwater production. The rate of water inflow was estimated from the dilution of the tracer in the drill fluid returns. However, loss of circulation and use of bentonite mud prevented monitoring of $\mathrm{LiBr}$ dilution in several intervals.

Samples of drilling effluent were tested onsite hourly for the presence of tritium, and every 8 hours for lead. The onsite monitoring results indicate that lead was undetectable (less than 50 parts per billion) during the entire drilling operation, and tritium remained at background levels (IT, 2001b). However, slightly elevated tritium levels (up to $5,028 \mathrm{pCi} / \mathrm{L}$ ) were detected in the depth interval 274.3 to $312.4 \mathrm{~m}$ (900 to $1,025 \mathrm{ft})$.

Before fluids are discharged from a sump through the overflow pipe, the UGTA FMP requires that a sample be collected from the sump and analyzed offsite to verify onsite monitoring data and demonstrate compliance with the FMP. Duplicate samples were collected from Sump \#1 on February 14, 2001, before the sump filled to the level of the overflow pipes, but the analytical results were not available before it was necessary to divert fluids from Sump \#1 to Sump \#2. A leaking (closed) valve released approximately 881 liters (200 gallons) to the ground surface at this time. While the sample was being analyzed, the bottom of the surface hole was reached and geophysical logging was begun. The analytical results obtained on February 16, 2001, showed that the sump fluids were within the parameters of the FMP criteria, and discharge of fluid from Sump \#1 was approved by NNSA/NSO. A sample was collected from Sump \#2 before approval was given by NNSA/NSO to transfer fluids from Sump \#2 to a borrow pit on the east side of the drill site. The results of this analysis indicated that the sample was within the parameters of the FMP criteria. Samples from both sumps were also collected and analyzed at the end of drilling operations. Water-quality data for all 5 sump samples are provided in Appendix B.

The results of analyses of samples of drilling fluid collected at Well ER-5-4 during drilling operations indicate that all fluid quality objectives were met, as shown on the fluid management reporting form dated September 25, 2001 (Appendix B). The form lists volumes of solids (drill cuttings) and fluids produced during well-construction operations, Stages I and II (i.e., vadose- and saturated-zone drilling only; well development and aquifer testing will be conducted as a separate initiative). The volume of solids produced was calculated using the diameter of the borehole (from caliper logs) and the depth drilled, and includes added volume attributed to a rock bulking factor. The volumes of fluids listed on the report are estimates of total fluid production, and do not account for any infiltration or evaporation of fluids from the sumps. 


\subsection{Geologic Data Collection}

This section describes the sources of geologic data obtained from Well ER-5-4 and the methods of data collection. Improving the understanding of variations within the alluvial section and gaining information on the volcanic rocks beneath the alluvium in this part of the Frenchman Flat basin were among the primary objectives of Well ER-5-4, so the proper collection of geologic and hydrogeologic data from Well ER-5-4 was considered fundamental to successful completion of the project. The geology of this site is presented in Section 4.0.

Geologic data collected at Well ER-5-4 consist of drill cuttings, sidewall core samples, and geophysical logs. Data collection, sampling, transfer, and documentation activities were performed according to applicable contractor procedures.

\subsubsection{Collection of Drill Cuttings}

Composite drill cuttings samples were collected from Well ER-5-4 at 3-m (10-ft) intervals as drilling progressed from the depth of $36.6 \mathrm{~m}(120 \mathrm{ft})$ to the TD of the well at $1,137 \mathrm{~m}(3,732 \mathrm{ft})$. Cuttings samples were collected from 301 intervals. The effort was made to obtain enough material for triplicate samples, each consisting of approximately 550 cubic centimeters (cc) (1 pint) of drill cuttings, in each 3-m (10-ft) sample interval. However, in the lower portion of the hole the volume of cuttings was low, and some samples are composites of materials from intervals as great as 9.1 to $21.3 \mathrm{~m}$ (30 to $70 \mathrm{ft}$ ). Also, no samples were collected in several intervals where drill fluid was not returned to the surface. The drill cuttings samples are stored under secure, environmentally controlled conditions at the U.S. Geological Survey (USGS) Geologic Data Center and Core Library in Mercury, Nevada. One of these sample sets was sealed with custody tape at the rig site and remains sealed as an archive sample; one set was left unsealed in the original sample containers; and the third set was washed and stored according to standard USGS Core Library procedures. The washed set was used by BN to construct the detailed lithologic log presented in Appendix C-1.

The IT field representative collected an additional 2 sets of reference drill cuttings samples (approximately $15 \mathrm{cc}$ ) from each of the sample intervals. One set was examined at the drill site for use in preparing field lithologic descriptions, and remains in the custody of IT (now Stoller-Navarro, IT and Shaw's successor and the current environmental contractor for NNSA/NSO). The other set was sent to R. G. Warren at LANL for petrographic, mineralogic, and chemical analyses, where it remains. 


\subsubsection{Sidewall Core Samples}

A Schlumberger percussion gun tool was used to collect sidewall core samples from 154 locations in Well ER-5-4. These samples were collected primarily to support a study by project scientists to characterize the distribution of reactive minerals within the alluvium in Frenchman Flat. The sidewall samples were also very valuable as sources of geologic data to supplement the generally poor cuttings samples. Sample locations were selected by the IT Field Representative on the basis of field lithologic logs (with consideration of borehole conditions determined from caliper logs). Eleven sidewall cores were obtained in the upper $231.6 \mathrm{~m}$ ( $760 \mathrm{ft}$ ) of the borehole on February 17, 2001, prior to installation of the surface casing. Prior to installation of the intermediate casing, Schlumberger collected 83 sidewall cores from the interval 290.8 to $506.0 \mathrm{~m}$ (954 to 1,660 ft). The final 60 sidewall samples were obtained from the lower part of the borehole before the completion string was installed. Table 2-2 1 ists the core length recovered for each sample depth.

\subsubsection{Sample Analysis}

Nine samples of drill cuttings and 154 sidewall core samples from various depths in Well ER-5-4 were submitted to the LANL Earth and Environmental Sciences Division - Geology and Geochemistry laboratories for petrographic, mineralogic, and chemical analyses. Table 2-3 lists sample depth and type, and the analyses performed on each sample. Analytical results and interpretations were incorporated into the detailed lithologic $\log$ for Well ER-5-4 presented in Appendix C of this report, and in the discussion of the geology of the well presented in Section 4.0. More detailed information on the analytical results and interpretations is provided in Warren et al. (2002).

\subsubsection{Geophysical Data}

Geophysical logs were run in the borehole to further characterize the lithology, structure, and water content of the rocks encountered. In addition, logs were run to evaluate borehole conditions, to determine the fluid levels during the course of drilling, and to monitor completion progress. Geophysical logging was conducted during 3 stages of drilling and completion: prior to setting surface casing, prior to installing the completion well casing, and during well installation (annulus investigation log). Some logs were run in both the saturated and unsaturated zones of the borehole, while others (e.g., thermal flow log, chemistry log, ultrasonic borehole imager log, etc.) were run only in the saturated interval. A complete listing of the logs, dates run, depths, and service companies is provided in Table 2-4. The logs are available from BN in Mercury, Nevada, and copies are on file at the Stoller-Navarro office in Las Vegas, Nevada. Preliminary geophysical data from the logs are reproduced in Appendix D. 


\section{Table 2-2}

Percussion Gun Sidewall Core Samples from Well ER-5-4

(Page 1 of 2)

\begin{tabular}{||c|c||}
\hline $\begin{array}{c}\text { Core Depth } \\
\text { meters (feet) }\end{array}$ & $\begin{array}{c}\text { Rength } \\
\text { cm (in.) }\end{array}$ \\
\hline \hline $192.0(630)$ & $4.06(1.6)$ \\
\hline $196.9(646)$ & $0.51(0.2)$ \\
\hline $202.4(664)$ & $0.25(0.1)$ \\
\hline $204.8(672)$ & $5.08(2.0)$ \\
\hline $210.3(690)$ & $5.08(2.0)$ \\
\hline $211.8(695)$ & $1.02(0.4)$ \\
\hline $214.0(702)$ & $1.52(0.6)$ \\
\hline $226.2(742)$ & $2.54(1.0)$ \\
\hline $227.7(747)$ & $2.03(0.8)$ \\
\hline $229.2(752)$ & $0.51(0.2)$ \\
\hline $231.6(760)$ & $2.54(1.0)$ \\
\hline $290.8(954)$ & $2.54(1.0)$ \\
\hline $296.3(972)$ & $2.54(1.0)$ \\
\hline $315.5(1,035)$ & $3.18(1.25)$ \\
\hline $316.1(1,037)$ & $2.54(1.0)$ \\
\hline $317.0(1,040)$ & $1.27(0.5)$ \\
\hline $318.5(1,045)$ & $3.18(1.25)$ \\
\hline $320.0(1,050)$ & $3.18(1.25)$ \\
\hline $321.9(1,056)$ & $3.81(1.5)$ \\
\hline $322.5(1,058)$ & $1.27(0.5)$ \\
\hline $323.1(1,060)$ & $2.54(1.0)$ \\
\hline $324.6(1,065)$ & $1.27(0.5)$ \\
\hline $326.1(1,070)$ & $3.18(1.25)$ \\
\hline $326.4(1,071)$ & $5.08(2.0)$ \\
\hline $327.7(1,072)$ & $3.81(1.5)$ \\
\hline 3.073$)$ & $5.08(2.0)$ \\
\hline 3.07$)$ & $5.08(2.0)$ \\
\hline 3275$)$
\end{tabular}

\begin{tabular}{|c|c|}
\hline $\begin{array}{l}\text { Core Depth } \\
\text { meters (feet) }\end{array}$ & $\begin{array}{c}\text { Length } \\
\text { Recovered } \\
\text { cm (in.) }\end{array}$ \\
\hline $328.0(1,076)$ & $3.18(1.25)$ \\
\hline $328.6(1,078)$ & $5.08(2.0)$ \\
\hline $329.2(1,080)$ & $4.45(1.75)$ \\
\hline $330.7(1,085)$ & $5.08(2.0)$ \\
\hline $332.2(1,090)$ & $1.91(0.75)$ \\
\hline $333.5(1,094)$ & $2.54(1.0)$ \\
\hline $333.8(1,095)$ & $1.91(0.75)$ \\
\hline $338.9(1,112)$ & $2.54(1.0)$ \\
\hline $339.5(1,114)$ & $5.08(2.0)$ \\
\hline $342.3(1,123)$ & $3.18(1.25)$ \\
\hline $352.0(1,155)$ & $1.27(0.5)$ \\
\hline $356.6(1,170)$ & $3.81(1.5)$ \\
\hline $359.7(1,180)$ & $2.54(1.0)$ \\
\hline $361.5(1,186)$ & $5.08(2.0)$ \\
\hline $367.0(1,204)$ & $.05(.02)$ \\
\hline $368.2(1,208)$ & $4.45(1.75)$ \\
\hline $368.8(1,210)$ & $5.08(2.0)$ \\
\hline $369.7(1,213)$ & $3.18(1.25)$ \\
\hline $374.9(1,230)$ & $3.81(1.5)$ \\
\hline $377.3(1,238)$ & $3.18(1.25)$ \\
\hline $385.9(1,266)$ & $1.27(0.5)$ \\
\hline $387.1(1,270)$ & $1.91(0.75)$ \\
\hline $390.4(1,281)$ & $1.27(0.5)$ \\
\hline $395.3(1,297)$ & $5.08(2.0)$ \\
\hline $396.2(1,300)$ & $3.81(1.5)$ \\
\hline $400.8(1,315)$ & $1.27(0.5)$ \\
\hline $406.6(1,334)$ & $3.81(1.5)$ \\
\hline $411.5(1,350)$ & $3.18(1.25)$ \\
\hline
\end{tabular}

\begin{tabular}{||c|c||}
\hline \hline $\begin{array}{c}\text { Core Depth } \\
\text { meters (feet) }\end{array}$ & $\begin{array}{c}\text { Recovered } \\
\text { cm (in.) }\end{array}$ \\
\hline \hline $413.0(1,355)$ & $1.91(0.75)$ \\
\hline $414.5(1,360)$ & $3.18(1.25)$ \\
\hline $415.4(1,363)$ & $4.45(1.75)$ \\
\hline $415.7(1,364)$ & $1.91(0.75)$ \\
\hline $416.1(1,365)$ & $2.54(1.0)$ \\
\hline $416.4(1,366)$ & $2.54(1.0)$ \\
\hline $416.7(1,367)$ & $1.91(0.75)$ \\
\hline $417.6(1,370)$ & $3.18(1.25)$ \\
\hline $418.8(1,374)$ & $4.45(1.75)$ \\
\hline $420.6(1,380)$ & $5.08(2.0)$ \\
\hline $422.1(1,385)$ & $1.27(0.5)$ \\
\hline $423.7(1,390)$ & $2.54(1.0)$ \\
\hline $425.2(1,395)$ & $1.27(0.5)$ \\
\hline $427.9(1,404)$ & $1.91(0.75)$ \\
\hline $428.2(1,405)$ & $2.54(1.0)$ \\
\hline $432.8(1,420)$ & $5.08(2.0)$ \\
\hline $434.0(1,424)$ & $5.08(2.0)$ \\
\hline $435.9(1,430)$ & $0.64(0.25)$ \\
\hline $437.4(1,435)$ & $2.54(1.0)$ \\
\hline $448.1(1,470)$ & $5.08(2.0)$ \\
\hline $451.1(1,480)$ & $3.18(1.25)$ \\
\hline $452.3(1,484)$ & $4.45(1.75)$ \\
\hline $457.2(1,500)$ & $5.08(2.0)$ \\
\hline $457.8(1,502)$ & $4.45(1.75)$ \\
\hline $460.2(1,510)$ & $5.08(2.0)$ \\
\hline $4.5(1,540)$ & $5.08(2.0)$ \\
\hline 4.550$)$ & $3.18(1.25)$ \\
\hline 40.75$)$ \\
\hline 467$)$
\end{tabular}


Table 2-2

Percussion Gun Sidewall Core Samples from Well ER-5-4

(Page 2 of 2)

\begin{tabular}{||c|c||}
\hline $\begin{array}{c}\text { Core Depth } \\
\text { meters (feet) }\end{array}$ & $\begin{array}{c}\text { Length } \\
\text { Recovered } \\
\text { cm (in.) }\end{array}$ \\
\hline \hline $475.5(1,560)$ & $1.91(0.75)$ \\
\hline $477.9(1,568)$ & $3.18(1.25)$ \\
\hline $483.1(1,585)$ & $2.54(1.0)$ \\
\hline $484.6(1,590)$ & $5.08(2.0)$ \\
\hline $487.7(1,600)$ & $2.54(1.0)$ \\
\hline $488.9(1,604)$ & $1.27(0.5)$ \\
\hline $493.8(1,620)$ & $5.08(2.0)$ \\
\hline $496.2(1,628)$ & $1.91(0.75)$ \\
\hline $502.9(1,650)$ & $5.08(2.0)$ \\
\hline $506.0(1,660)$ & $3.18(1.25)$ \\
\hline $614.5(2,016)$ & $4.45(1.75)$ \\
\hline $623.9(2,047)$ & $4.45(1.75)$ \\
\hline $641.6(2,105)$ & $5.08(2.0)$ \\
\hline $654.1(2,146)$ & $5.08(2.0)$ \\
\hline $673.6(2,210)$ & $5.08(2.0)$ \\
\hline $686.4(2,252)$ & $5.08(2.0)$ \\
\hline $707.1(2,320)$ & $5.08(2.0)$ \\
\hline $714.5(2,344)$ & $5.08(2.0)$ \\
\hline $730.9(2,398)$ & $4.45(1.75)$ \\
\hline $737.9(2,421)$ & $4.45(1.75)$ \\
\hline $748.0(2,454)$ & $3.81(1.5)$ \\
\hline $762.6(2,502)$ & $5.08(2.0)$ \\
\hline $768.7(2,522)$ & $3.18(1.25)$ \\
\hline $773.0(2,536)$ & $5.08(2.0)$ \\
\hline \hline
\end{tabular}

\begin{tabular}{||c|c||}
\hline \hline $\begin{array}{c}\text { Core Depth } \\
\text { meters (feet) }\end{array}$ & $\begin{array}{c}\text { Length } \\
\text { Recovered } \\
\text { cm. }\end{array}$ \\
\hline \hline $776.0(2,546)$ & $3.56(1.4)$ \\
\hline $780.0(2,559)$ & $4.06(1.6)$ \\
\hline $792.8(2,601)$ & $5.08(2.0)$ \\
\hline $801.0(2,628)$ & $5.08(2.0)$ \\
\hline $809.9(2,657)$ & $4.45(1.75)$ \\
\hline $816.9(2,680)$ & $5.08(2.0)$ \\
\hline $821.1(2,694)$ & $5.08(2.0)$ \\
\hline $824.2(2,704)$ & $4.06(1.6)$ \\
\hline $831.5(2,728)$ & $5.08(2.0)$ \\
\hline $835.2(2,740)$ & $5.08(2.0)$ \\
\hline $846.7(2,778)$ & $5.08(2.0)$ \\
\hline $851.0(2,792)$ & $5.08(2.0)$ \\
\hline $856.5(2,810)$ & $5.08(2.0)$ \\
\hline $859.5(2,820)$ & $5.08(2.0)$ \\
\hline $862.6(2,830)$ & $5.08(2.0)$ \\
\hline $871.7(2,860)$ & $4.45(1.75)$ \\
\hline $877.8(2,880$ & $4.45(1.75)$ \\
\hline $887.0(2,910)$ & $5.08(2.0)$ \\
\hline $902.2(2,960)$ & $3.81(1.5)$ \\
\hline $914.4(3,000)$ & $3.18(1.25)$ \\
\hline $922.3(3,026)$ & $5.08(2.0)$ \\
\hline $928.7(3,047)$ & $4.06(1.6)$ \\
\hline 9.077$)$ & $4.45(1.75)$ \\
\hline $89.9(3)$
\end{tabular}

\begin{tabular}{||c|c||}
\hline $\begin{array}{c}\text { Core Depth } \\
\text { meters (feet) }\end{array}$ & $\begin{array}{c}\text { Length } \\
\text { Recovered } \\
\text { cm (in.) }\end{array}$ \\
\hline \hline $949.5(3,115)$ & $4.06(1.6)$ \\
\hline $960.4(3,151)$ & $5.08(2.0)$ \\
\hline $971.1(3,186)$ & $0.05(0 . .2)$ \\
\hline $979.6(3,214)$ & $4.45(1.75)$ \\
\hline $990.6(3,250)$ & $5.08(2)$ \\
\hline $999.7(3,280)$ & $3.81(1.5)$ \\
\hline $1,006.4(3,302)$ & $4.32(1.7)$ \\
\hline $1,013.5(3,325)$ & $5.08(2.0)$ \\
\hline $1,024.4(3,361)$ & $1.91(0.75)$ \\
\hline $1,031.4(3,384)$ & $3.81(1.5)$ \\
\hline $1,036.3(3,400)$ & $5.08(2.0)$ \\
\hline $1,041.5(3,417)$ & $5.08(2.0)$ \\
\hline $1,044.9(3,428)$ & $0.51(0.2)$ \\
\hline $1,051.6(3,450)$ & $4.45(1.75)$ \\
\hline $1,058.3(3,472)$ & $3.81(1.5)$ \\
\hline $1,060.7(3,480)$ & $3.56(1.4)$ \\
\hline $1,064.4(3,492)$ & $3.18(1.25)$ \\
\hline $1,067.4(3,502)$ & $3.81(1.5)$ \\
\hline $1,075.9(3,530)$ & $5.08(2.0)$ \\
\hline $1,081.1(3,547)$ & $2.54(1.0)$ \\
\hline $1,083.3(3,554)$ & $5.08(2.0)$ \\
\hline $1,086.9(3,566)$ & $3.18(1.25)$ \\
\hline $1,091.2(3,580)$ & $5.08(2.0)$ \\
\hline \hline
\end{tabular}

All samples are Quaternary or Tertiary alluvium. 
Table 2-3

Status of Rock Sample Analyses for Well ER-5-4

\begin{tabular}{|c|c|c|c|c|c|c|c|}
\hline \multirow{3}{*}{$\begin{array}{c}\text { Depth } \\
\text { meters (feet) }\end{array}$} & \multirow{3}{*}{$\begin{array}{l}\text { Sample } \\
\text { Type }^{\text {b }}\end{array}$} & \multicolumn{6}{|c|}{ Analyses Performed $^{c}$} \\
\hline & & \multicolumn{2}{|c|}{ Petrographic } & \multicolumn{2}{|c|}{ Mineralogic } & \multicolumn{2}{|c|}{ Chemical } \\
\hline & & PTS & SEM & MP & XRD & XRF & $\mathrm{Fe}^{2+} / \mathrm{Fe}^{3+}$ \\
\hline $48.8(160)$ & $\mathrm{DC}$ & $\mathrm{C}$ & -- & -- & -- & -- & -- \\
\hline $192.0(630)$ & SWC & C & -- & -- & C & C & C \\
\hline $204.8(672)$ & SWC & -- & -- & -- & C & C & $\mathrm{C}$ \\
\hline $231.6(760)$ & SWC & $\mathrm{C}$ & C & $\mathrm{C}$ & C & -- & -- \\
\hline $286.5(940)$ & $\mathrm{DC}$ & C & -- & -- & -- & -- & -- \\
\hline $315.5(1,035)$ & SWC & -- & C & -- & C & -- & -- \\
\hline $317.0(1,040)$ & SWC & -- & C & -- & C & -- & -- \\
\hline $318.5(1,045)$ & SWC & -- & C & -- & C & -- & -- \\
\hline $320.0(1,050)$ & SWC & -- & C & -- & C & -- & -- \\
\hline $321.9(1,056)$ & SWC & -- & $\mathrm{C}$ & -- & $\mathrm{C}$ & -- & -- \\
\hline $323.1(1,060)$ & SWC & C & C & C & C & -- & -- \\
\hline $324.6(1,065)$ & SWC & -- & C & -- & C & -- & -- \\
\hline $326.1(1,071)$ & SWC & -- & C & -- & C & -- & -- \\
\hline $326.7(1,072)$ & SWC & C & C & C & C & -- & -- \\
\hline $327.1(1,073)$ & SWC & -- & C & -- & C & -- & -- \\
\hline $327.4(1,074)$ & SWC & -- & $x$ & -- & C & -- & -- \\
\hline $327.7(1,075)$ & SWC & -- & C & -- & C & -- & -- \\
\hline $328.0(1,076)$ & SWC & C & C & C & C & -- & -- \\
\hline $328.6(1.078)$ & SWC & -- & C & -- & C & -- & -- \\
\hline $329.2(1,080)$ & SWC & C & C & C & C & -- & -- \\
\hline $330.7(1,085)$ & SWC & -- & C & -- & C & -- & -- \\
\hline $332.2(1,090)$ & SWC & -- & C & -- & C & -- & -- \\
\hline $333.8(1,095)$ & SWC & -- & C & -- & C & -- & -- \\
\hline $339.5(1,114)$ & SWC & -- & C & -- & C & -- & -- \\
\hline $352.0(1,155)$ & SWC & C & C & C & C & -- & -- \\
\hline $359.7(1,180)$ & SWC & -- & C & -- & C & -- & -- \\
\hline $368.8(1,210)$ & SWC & C & C & C & C & -- & -- \\
\hline
\end{tabular}


Table 2-3

Status of Rock Sample Analyses for Well ER-5-4 (continued)

\begin{tabular}{|c|c|c|c|c|c|c|c|}
\hline \multirow{3}{*}{$\begin{array}{c}\text { Depth } \\
\text { meters (feet) }{ }^{a}\end{array}$} & \multirow{3}{*}{$\begin{array}{l}\text { Sample } \\
\text { Type }^{\text {b }}\end{array}$} & \multicolumn{6}{|c|}{ Analyses Performed $^{c}$} \\
\hline & & \multicolumn{2}{|c|}{ Petrographic } & \multicolumn{2}{|c|}{ Mineralogic } & \multicolumn{2}{|c|}{ Chemical } \\
\hline & & PTS & SEM & MP & XRD & XRF & $\mathrm{Fe}^{2+} / \mathrm{Fe}^{3+}$ \\
\hline $377.3(1,238)$ & SWC & -- & $\mathrm{C}$ & -- & $\mathrm{C}$ & -- & -- \\
\hline $387.1(1,270)$ & SWC & -- & $\mathrm{C}$ & -- & $\mathrm{C}$ & -- & -- \\
\hline $396.2(1,300)$ & SWC & -- & $\mathrm{C}$ & -- & C & -- & -- \\
\hline $406.6(1,334)$ & SWC & C & C & C & C & -- & -- \\
\hline $411.5(1,350)$ & SWC & -- & $\mathrm{C}$ & -- & C & -- & -- \\
\hline $413.0(1,355)$ & SWC & -- & $\mathrm{C}$ & -- & $\mathrm{C}$ & -- & -- \\
\hline $414.5(1,360)$ & SWC & -- & C & -- & C & -- & -- \\
\hline $415.4(1,363)$ & SWC & -- & C & -- & C & -- & -- \\
\hline $415.7(1,364)$ & SWC & -- & $\mathrm{C}$ & -- & C & -- & -- \\
\hline $416.1(1,365)$ & SWC & -- & $x$ & -- & C & -- & -- \\
\hline $416.4(1,366)$ & SWC & C & C & -- & C & -- & -- \\
\hline $416.7(1,367)$ & SWC & -- & $x$ & -- & C & -- & -- \\
\hline $417.6(1,370)$ & SWC & -- & C & -- & C & -- & -- \\
\hline $418.8(1,374)$ & SWC & -- & C & -- & C & -- & -- \\
\hline $420.6(1,380)$ & SWC & -- & $x$ & -- & C & -- & -- \\
\hline $422.1(1,385)$ & SWC & -- & $x$ & -- & C & -- & -- \\
\hline $423.7(1,390)$ & SWC & -- & C & -- & C & -- & -- \\
\hline $425.2(1,395)$ & SWC & $\mathrm{C}$ & C & C & C & -- & -- \\
\hline $427.9(1,404)$ & SWC & C & C & C & C & -- & -- \\
\hline $428.2(1,405)$ & SWC & -- & C & -- & C & -- & -- \\
\hline $432.8(1,420)$ & SWC & $\mathrm{C}$ & $\mathrm{C}$ & $\mathrm{C}$ & $\mathrm{C}$ & -- & -- \\
\hline $437.4(1,435)$ & SWC & C & -- & -- & C & C & C \\
\hline $451.1(1,480)$ & SWC & C & $\mathrm{C}$ & -- & $\mathrm{C}$ & -- & -- \\
\hline $457.2(1,500)$ & SWC & -- & C & -- & C & -- & -- \\
\hline $469.4(1,540)$ & SWC & -- & -- & -- & $\mathrm{C}$ & C & C \\
\hline $477.9(1,568)$ & SWC & -- & -- & -- & $\mathrm{C}$ & C & C \\
\hline $487.7(1,600)$ & SWC & C & C & C & C & -- & -- \\
\hline $493.8(1,620)$ & SWC & -- & -- & -- & C & C & C \\
\hline
\end{tabular}


Table 2-3

Status of Rock Sample Analyses for Well ER-5-4 (continued)

\begin{tabular}{|c|c|c|c|c|c|c|c|}
\hline \multirow{3}{*}{$\begin{array}{c}\text { Depth } \\
\text { meters (feet) }{ }^{a}\end{array}$} & \multirow{3}{*}{$\begin{array}{c}\text { Sample } \\
\text { Type }^{\text {b }}\end{array}$} & \multicolumn{6}{|c|}{ Analyses Performed $^{c}$} \\
\hline & & \multicolumn{2}{|c|}{ Petrographic } & \multicolumn{2}{|c|}{ Mineralogic } & \multicolumn{2}{|c|}{ Chemical } \\
\hline & & PTS & SEM & MP & XRD & XRF & $\mathrm{Fe}^{2+} / \mathrm{Fe}^{3+}$ \\
\hline $502.9(1,650)$ & SWC & -- & -- & -- & C & C & C \\
\hline $521.2(1,710)$ & $\mathrm{DC}$ & -- & -- & -- & $\mathrm{C}$ & $\mathrm{C}$ & $\mathrm{C}$ \\
\hline $536.4(1,760)$ & DC & -- & -- & -- & C & $\mathrm{C}$ & C \\
\hline $570.0(1,870)$ & $\mathrm{DC}$ & -- & -- & -- & C & C & C \\
\hline $609.6(2,000)$ & DC & -- & -- & -- & C & C & $\mathrm{C}$ \\
\hline $614.5(2,016)$ & SWC & $\mathrm{C}$ & C & C & C & -- & -- \\
\hline $623.9(2,047)$ & SWC & -- & -- & -- & C & C & C \\
\hline $641.6(2,105)$ & SWC & C & $x$ & C & C & -- & -- \\
\hline $654.1(2,146)$ & SWC & -- & -- & -- & C & C & C \\
\hline $673.6(2,210)$ & SWC & C & C & C & C & -- & -- \\
\hline $686.4(2,252)$ & SWC & -- & -- & -- & C & C & C \\
\hline $707.1(2,320)$ & SWC & -- & C & -- & C & -- & -- \\
\hline $730.9(2,398)$ & SWC & C & $\mathrm{C}$ & -- & C & -- & -- \\
\hline $748.0(2,454)$ & SWC & $\mathrm{C}$ & -- & -- & $\mathrm{C}$ & $\mathrm{C}$ & $\mathrm{C}$ \\
\hline $762.6(2,502)$ & SWC & C & C & -- & C & -- & -- \\
\hline $776.0(2,546)$ & SWC & -- & -- & -- & C & C & C \\
\hline $792.8(2,601)$ & SWC & C & C & C & C & -- & -- \\
\hline $809.9(2,657)$ & swc & $\mathrm{C}$ & -- & -- & C & $\mathrm{C}$ & $\mathrm{C}$ \\
\hline $821.1(2,694)$ & swc & -- & $\mathrm{C}$ & -- & C & -- & -- \\
\hline $824.2(2,704)$ & SWC & $\mathrm{C}$ & -- & -- & C & $\mathrm{C}$ & $\mathrm{C}$ \\
\hline $835.2(2,740)$ & SWC & -- & -- & -- & $\mathrm{C}$ & C & $\mathrm{C}$ \\
\hline $856.5(2,810)$ & SWC & -- & C & -- & C & -- & -- \\
\hline $871.7(2,860)$ & SWC & -- & -- & -- & C & C & C \\
\hline $887.0(2,910)$ & SWC & C & C & -- & C & -- & -- \\
\hline $902.2(2,960)$ & SWC & -- & -- & -- & C & C & C \\
\hline $914.4(3,000)$ & SWC & -- & C & -- & C & -- & -- \\
\hline $928.7(3,047)$ & SWC & C & -- & -- & C & C & C \\
\hline $949.5(3,115)$ & SWC & -- & C & -- & C & -- & -- \\
\hline
\end{tabular}


Table 2-3

Status of Rock Sample Analyses for Well ER-5-4 (continued)

\begin{tabular}{|c|c|c|c|c|c|c|c|}
\hline \multirow{3}{*}{$\begin{array}{c}\text { Depth } \\
\text { meters (feet) a }\end{array}$} & \multirow{3}{*}{$\begin{array}{c}\text { Sample } \\
\text { Type }^{\text {b }}\end{array}$} & \multicolumn{6}{|c|}{ Analyses Performed $^{\mathrm{c}}$} \\
\hline & & \multicolumn{2}{|c|}{ Petrographic } & \multicolumn{2}{|c|}{ Mineralogic } & \multicolumn{2}{|c|}{ Chemical } \\
\hline & & PTS & SEM & MP & XRD & XRF & $\mathrm{Fe}^{2+} / \mathrm{Fe}^{3+}$ \\
\hline $960.4(3,151)$ & SWC & -- & -- & -- & C & C & C \\
\hline $979.6(3,214)$ & SWC & C & C & C & C & -- & -- \\
\hline $990.6(3,250)$ & SWC & -- & -- & -- & $\mathrm{C}$ & $\mathrm{C}$ & $\mathrm{C}$ \\
\hline $1,006.4(3,302)$ & swc & C & C & C & C & -- & -- \\
\hline $1,024.4(3,361)$ & SWC & C & -- & -- & C & C & C \\
\hline $1,036.3(3,400)$ & swc & -- & $\mathrm{C}$ & -- & C & -- & -- \\
\hline $1,051.6(3,450)$ & SWC & -- & -- & -- & C & C & C \\
\hline $1,067.4(3,502)$ & SWC & C & C & C & C & -- & -- \\
\hline $1,083.3(3,554)$ & SWC & C & -- & -- & C & $\mathrm{C}$ & $C$ \\
\hline $1,091.2(3,580)$ & SWC & -- & -- & -- & C & C & C \\
\hline $1,103.4(3,620)$ & $\mathrm{DC}$ & -- & -- & -- & C & C & C \\
\hline $1,118.6(3,670)$ & $\mathrm{DC}$ & -- & -- & -- & C & C & $\mathrm{C}$ \\
\hline $1,133.9(3,720)$ & $\mathrm{DC}$ & C & -- & -- & C & $\mathrm{C}$ & C \\
\hline
\end{tabular}

a Depth represents base of 3-m (10-ft) interval for drill cuttings.

b $\quad$ DC $=$ drill cuttings sample; $\mathbf{S W C}=$ sidewall core sample.

C Status of analyses: $\mathbf{C}=$ analysis complete; $\mathbf{X}=$ SEM results were not obtained or were poor, due to surface charging; -- = analysis not performed. Analysis type: PTS = polished thin section; $\mathbf{S E M}=$ scanning electron microscopy; $\mathbf{M P}=$ electron microprobe; $\mathbf{X R D}=\mathrm{x}$-ray diffraction; $\mathbf{X R F}=\mathrm{X}$-ray fluorescence; $\mathbf{F e}^{2+} / \mathrm{Fe}^{3+}=\mathrm{wet}$ chemical analysis for iron. 
Table 2-4

Well ER-5-4 Geophysical Log Summary

(Page 1 of 2)

\begin{tabular}{|c|c|c|c|c|c|c|}
\hline Geophysical Log Type ${ }^{a}$ & Log Purpose & $\begin{array}{l}\text { Logging } \\
\text { Service }\end{array}$ & $\begin{array}{l}\text { Date } \\
\text { Logged }\end{array}$ & Run Number & $\begin{array}{l}\text { Bottom of } \\
\text { Logged } \\
\text { Interval }^{b} \\
\text { meters (feet) }\end{array}$ & $\begin{array}{l}\text { Top of Logged } \\
\text { Interval } \\
\text { meters (feet) }\end{array}$ \\
\hline * Natural Gamma Ray Spectroscopy & $\begin{array}{l}\text { Stratigraphic correlation, } \\
\text { mineralogy, natural and man- } \\
\text { made radiation, lithologic } \\
\text { determination, fracture } \\
\text { identification }\end{array}$ & Schlumberger & $\begin{array}{l}02 / 17 / 2001 \\
03 / 09 / 2001 \\
03 / 25 / 2001\end{array}$ & $\begin{array}{l}\text { SGR-1 } \\
\text { SGR-2 } \\
\text { SGR-3 }\end{array}$ & $\begin{array}{c}243.2(798) \\
496.8(1,630) \\
1,090.6(3,578)\end{array}$ & $\begin{array}{c}36.3(199) \\
194.5(638) \\
460.2(1,510)\end{array}$ \\
\hline * Four Arm Caliper/ Gamma Ray & $\begin{array}{l}\text { Borehole conditions, cement } \\
\text { volume calculation, fracture } \\
\text { identification }\end{array}$ & Schlumberger & $\begin{array}{l}02 / 16 / 2001 \\
03 / 09 / 2001 \\
03 / 19 / 2001 \\
03 / 20 / 2001 \\
03 / 24 / 2001\end{array}$ & $\begin{array}{l}\text { CA4-1/GR-1 } \\
\text { CA4-2/SGR-2 } \\
\text { CA4-3/GR-4 } \\
\text { CA4-4/GR-5 } \\
\text { CA4-5/GR-6 }\end{array}$ & $\begin{array}{c}252.1(827) \\
508.7(1,669) \\
651.1(2,136) \\
779.7(2,558) \\
1,099.7(3,608)\end{array}$ & $\begin{array}{c}36.3(119) \\
194.5(638) \\
493.8(1,620) \\
491.6(1,613) \\
492.6(1,616)\end{array}$ \\
\hline $\begin{array}{l}\text { *Array Induction Log/Spontaneous } \\
\text { Potential }\end{array}$ & $\begin{array}{l}\text { Lithologic determination, } \\
\text { stratigraphic correlation }\end{array}$ & Schlumberger & $\begin{array}{l}02 / 16 / 2001 \\
03 / 09 / 2001\end{array}$ & $\begin{array}{l}\text { AIT-1/SP-1/GR-2 } \\
\text { AIT-2/ SP-2/GR-3 }\end{array}$ & $\begin{array}{c}250.0(820) \\
505.4(1,658)\end{array}$ & $\begin{array}{l}36.3(119) \\
235.3(772)\end{array}$ \\
\hline * Epithermal Neutron/ Density & $\begin{array}{l}\text { Total water content, rock } \\
\text { porosity, stratigraphic correlation, } \\
\text { lithologic determination }\end{array}$ & Schlumberger & $\begin{array}{l}02 / 16 / 2001 \\
03 / 09 / 2001 \\
03 / 25 / 2001\end{array}$ & $\begin{array}{l}\text { ENP-1/CDL-1/GR-2 } \\
\text { ENP-2/CDL-2/GR-3 } \\
\text { ENP-3/CDL-3/GR-7 }\end{array}$ & $\begin{array}{c}246.9(810) \\
502.3(1,648) \\
1,090.3(3,577)\end{array}$ & $\begin{array}{c}36.6(119) \\
235.3(772) \\
395.6(1,298)\end{array}$ \\
\hline $\begin{array}{l}\text { * Dual Laterolog/ Spontaneous } \\
\text { Potential }\end{array}$ & $\begin{array}{l}\text { Water saturation, stratigraphic } \\
\text { correlation, lithologic } \\
\text { determination }\end{array}$ & Schlumberger & 03/25/2001 & DLL-1/ SP-3/GR-7 & $1,093.3(3,587)$ & $509.9(1,673)$ \\
\hline $\begin{array}{l}\text { Digital Array Sonic - wave-form and } \\
\text { variable density }\end{array}$ & Fracture identification & Schlumberger & 03/25/2001 & AC-1/ SGR-3 & $1,090.6(3,578)$ & $460.3(1,510)$ \\
\hline $\begin{array}{l}\text { *Digital Array Sonic - porosity and } \\
\text { travel time }\end{array}$ & Rock porosity, seismic properties & Schlumberger & 03/25/2001 & AC-1/ SGR-3 & $1,090.6(3,578)$ & $460.3(1,510)$ \\
\hline Ultrasonic Borehole Imager & $\begin{array}{l}\text { Lithologic characterization, } \\
\text { structural analysis, fracture } \\
\text { identification }\end{array}$ & Schlumberger & 03/26/2001 & BHTV-1 & $1,082.0(3,550)$ & $509.9(1,673)$ \\
\hline Temperature & $\begin{array}{l}\text { Groundwater temperature, open } \\
\text { fracture identification }\end{array}$ & Schlumberger & $\begin{array}{l}03 / 19 / 2001 \\
03 / 20 / 2001 \\
03 / 24 / 2001 \\
03 / 26 / 2001\end{array}$ & $\begin{array}{l}\text { TL-1/GR-4 } \\
\text { TL-2/GR-5 } \\
\text { TL-3/GR-6 } \\
\text { TL-4/GR-8 }\end{array}$ & $\begin{array}{c}638.3(2,094) \\
765.1(2,510) \\
1,087.8(3,569) \\
1,091.0(3,579)\end{array}$ & $\begin{array}{l}480.1(1,575) \\
482.2(1,582) \\
486.5(1,596) \\
487.8(1,600)\end{array}$ \\
\hline
\end{tabular}


Table 2-4

Well ER-5-4 Geophysical Log Summary

(Page 2 of 2)

\begin{tabular}{|c|c|c|c|c|c|c|}
\hline Geophysical Log Type ${ }^{a}$ & Log Purpose & $\begin{array}{l}\text { Logging } \\
\text { Service }\end{array}$ & $\begin{array}{l}\text { Date } \\
\text { Logged }\end{array}$ & Run Number & $\begin{array}{c}\text { Bottom of } \\
\text { Logged } \\
\text { Interval }^{\mathbf{b}} \\
\text { meters (feet) }\end{array}$ & $\begin{array}{l}\text { Top of Logged } \\
\text { Interval } \\
\text { meters (feet) }\end{array}$ \\
\hline Percussion Sidewall Coring Tool & Geologic Samples & Schlumberger & $\begin{array}{l}02 / 17 / 2001 \\
03 / 09 / 2001 \\
03 / 26 / 2001\end{array}$ & $\begin{array}{c}\text { SGUN-1 } \\
\text { SGUN-3, 4, } 5 \\
\text { SGUN-6, } 7\end{array}$ & $\begin{array}{c}231.7(760) \\
506.0(1,660) \\
1,091.2(3,580)\end{array}$ & $\begin{array}{c}184.7(606) \\
290.8(954) \\
614.5(2,016)\end{array}$ \\
\hline Thermal Flow Log & $\begin{array}{l}\text { Rate and direction of groundwater } \\
\text { flow in borehole }\end{array}$ & $\begin{array}{l}\text { Desert } \\
\text { Research } \\
\text { Institute }\end{array}$ & $07 / 13 / 2001$ & TFM-1 & $1,025.7(3,365)$ & $518.2(1,700)$ \\
\hline Chemistry/Temperature Log & $\begin{array}{l}\text { Groundwater chemistry and } \\
\text { temperature, formation } \\
\text { transmissivity }\end{array}$ & $\begin{array}{l}\text { Desert } \\
\text { Research } \\
\text { Institute }\end{array}$ & $07 / 13 / 2001$ & Chem-1/TL-5 & $1,046.4(3,433)$ & $221.6(727)$ \\
\hline Nuclear Annulus Investigation Log & Well construction monitoring & Colog & $\begin{array}{c}03 / 28-31 \\
2001\end{array}$ & AIN-1 & $1,047.9(3,438)$ & $495.3(1,625)$ \\
\hline Gyroscopic Directional Survey & $\begin{array}{l}\text { Bottom hole location, true vertical } \\
\text { depth }\end{array}$ & Gyrodata & 05/10/2001 & GDS-1 & $951.6(3,122)$ & 0 \\
\hline
\end{tabular}

a Logs presented in geophysical log summary, Appendix D, are indicated by *.

b Depth below ground surface. 


\section{$2.4 \quad H y d r o l o g y$ of Well ER-5-4}

Well ER-5-4 is located in central Frenchman Flat near the southernmost of two areas of underground nuclear test locations. Hydrologic data in this area are concentrated along a north-south band in the center of the valley, but various workers have developed potentiometric maps and hydrologic interpretations for the area from these previously existing data. These interpretations were used to estimate the expected water level at Well ER-5-4 and to plan for handling water production, as described in IT (2000, 2001a). The two closest wells to Well Cluster ER-5-4 are Wells RNM-1 and RNM-2, located $295.7 \mathrm{~m}$ (970 ft) west-southwest.

\subsubsection{Preliminary Water-Level Information}

The elevation of the water table at Well ER-5-4 was projected to be approximately $733.7 \mathrm{~m}(2,407 \mathrm{ft})$, as derived from sparse hydrologic data for this region (IT, 2000). Based on the pre-construction estimate of surface elevation at the site, depth to water was expected at approximately $219.4 \mathrm{~m}$ (720 ft) (IT, 2000). Fluid depths between 185.9 and $221.3 \mathrm{~m}$ (610 and $726 \mathrm{ft}$ ) were obtained from various geophysical logs run on February 16 and 19, 2001, and March 25 and 26, 2001, before the completion string was installed. The most reliable of these measurements, made before the introduction of heavy bentonite mud into the borehole, was $221.3 \mathrm{~m}$ (726 ft) obtained on February 16, 2001, when the borehole was at the depth of $312.1 \mathrm{~m}$ (1,024 ft) (IT, 2001b). Based on this preliminary fluid depth and the as-built surface elevation of $954.5 \mathrm{~m}(3,131.7 \mathrm{ft})$, the fluid level elevation at Well ER-5-4 is $733.3 \mathrm{~m}(2,405.7 \mathrm{ft})$. This is approximately $0.4 \mathrm{~m}(1.3 \mathrm{ft})$ below the predicted elevation of $734 \mathrm{~m}(2,407 \mathrm{ft})$. A transducer for monitoring of the water level was not installed at the time of completion.

\subsubsection{Water Production}

Water production was estimated during drilling of Well ER-5-4 on the basis of LiBr dilution data as measured by IT field personnel. Measurable water production (approximately 19 lpm [5 gpm]) began when the borehole had reached the depth of about $223.1 \mathrm{~m}(732 \mathrm{ft})$. The production rate increased rapidly to about 1,514 lpm (400 gpm) at the depth of about $305 \mathrm{~m}(1,000 \mathrm{ft})$. Fluid circulation was lost at the depth of $312.1 \mathrm{~m}(1,024 \mathrm{ft})$. Because of borehole instability problems, the hole was cased to the depth of $279.0 \mathrm{~m}(915.5 \mathrm{ft})$, and then finally cemented up to the depth of $273.1 \mathrm{~m}(896 \mathrm{ft})$ and re-drilled, then cased again to the depth of $510.0 \mathrm{~m}(1,673.3 \mathrm{ft})$. Water production was not measured during these operations. When drilling resumed, water production could be measured, and increased to approximately 2,271 lpm (600 gpm) at the depth of about $310 \mathrm{~m}(2,000 \mathrm{ft})$. Below that depth, most of the drill fluid was lost to the formation, and no water was produced from the borehole. All measurable water production 
was from the alluvial aquifer. Estimated water production rates are presented graphically in Appendix A-1.

\subsubsection{Preliminary Flow Meter and Chemistry Log Data}

Flow meter data, along with temperature, electrical conductivity (EC), and $\mathrm{pH}$ measurements, can be used to characterize borehole fluid variability, which may indicate inflow and outflow zones. Typically, these measurements are made before the completion string is installed, and the data are consulted during planning of zones to be completed. At Well ER-5-4, these data were collected approximately $3 \frac{1}{2} 2$ months after the completion string was installed, following partial development of the well by pumping (these pumping operations will be described in a separate report). Desert Research Institute (DRI) personnel made measurements inside the Well ER-5-4 completion string with their thermal flow meter (TFM) and chemistry tools on July 13, 2001.

DRI personnel obtained TFM measurements at 7 locations between the depths of 541.0 and 1,019.6 m $(1,775$ and 3,345 ft). Preliminary analysis of these data indicates a downward flow of water within the borehole at all 7 stations.

In addition, DRI ran a chemistry log, which included measurements of temperature, EC, and $\mathrm{pH}$, from 221.6 to $1,046.4 \mathrm{~m}$ ( 727 to $3,433 \mathrm{ft}$ ). Groundwater temperature gradually increased from the minimum reading of 23.2 degrees Celsius (C) (73.8 degrees Fahrenheit [F]) at the top of the fluid column to the deepest logged depth near the bottom of the completion string. The maximum temperature of 33.6 degrees $\mathrm{C}$ (92.5 degrees $\mathrm{F}$ ) was measured at 1,046.1 $\mathrm{m}(3,432 \mathrm{ft})$. A slight perturbation in the temperature curve was noted near the top of the uppermost cement section around the $5 \frac{1}{2}$-in. production casing.

\subsubsection{Preliminary Groundwater Characterization Samples}

No preliminary groundwater characterization samples were collected from Well ER-5-4 after drilling, because of the residual bentonite mud in the borehole, and because it was expected that samples would soon be collected during upcoming development and testing operations.

\subsection{Precompletion and Open-Hole Development}

No precompletion development was conducted in Well ER-5-4 due to borehole instability problems that persisted through geophysical logging operations and installation of the completion string. 


\subsection{Well Completion}

Well completion refers to the installation in a borehole of a string of pipe (casing or tubing) that is slotted or screened at one or more locations along its length. The completion process also typically includes emplacement of backfill materials around the casing, with coarse fill such as gravel adjacent to the open intervals and impervious materials such as cement between the open intervals to isolate them. The casing serves as a conduit for insertion of a pump in the well, for inserting devices for measuring fluid level, and for sampling, so that accurate potentiometric and water chemistry data can be collected from known portions of the borehole.

Installation of the completion string and packing materials at Well ER-5-4 took place on March 27 to 31, 2001 (a pump was installed later to support hydrologic testing, which is documented elsewhere). Figure 2-2 is a schematic of the final well-completion design for Well ER-5-4, Figure 2-3 shows a plan view and profile of the wellhead surface completion, and Table 2-5 is a construction summary for the well. Data for this section were obtained from daily operations and activity reports, casing records, and cementing records provided by the BN Drilling Department. Information from IT's well data report (IT, 2001b) was also consulted for preparation of this section.

\subsubsection{Well Completion Design}

The final completion design differs slightly from the proposed design, as described in the following paragraphs.

\subsubsection{Proposed Completion Design}

The proposed completion design (IT, 2001a) was based on the assumption that Well ER-5-4 would penetrate a thick alluvial aquifer and reach TD in older Tertiary volcanic and volcaniclastic rocks of the tuff confining unit. The well was planned to be constructed with 2 completion zones, one near the base of the alluvial section, and the other in the middle of the saturated portion of the alluvium, with a piezometer installed just below the static water level. A string of 51/2-in. stainless-steel casing with 2 slotted intervals would be installed to provide access to the lower and middle saturated alluvium. A string of $2 f$-in. tubing would be installed outside the production casing, just below the static water level, to serve as a piezometer. The primary goal was to obtain separate completion zones within the top, middle, and bottom of the saturated alluvial aquifer. 


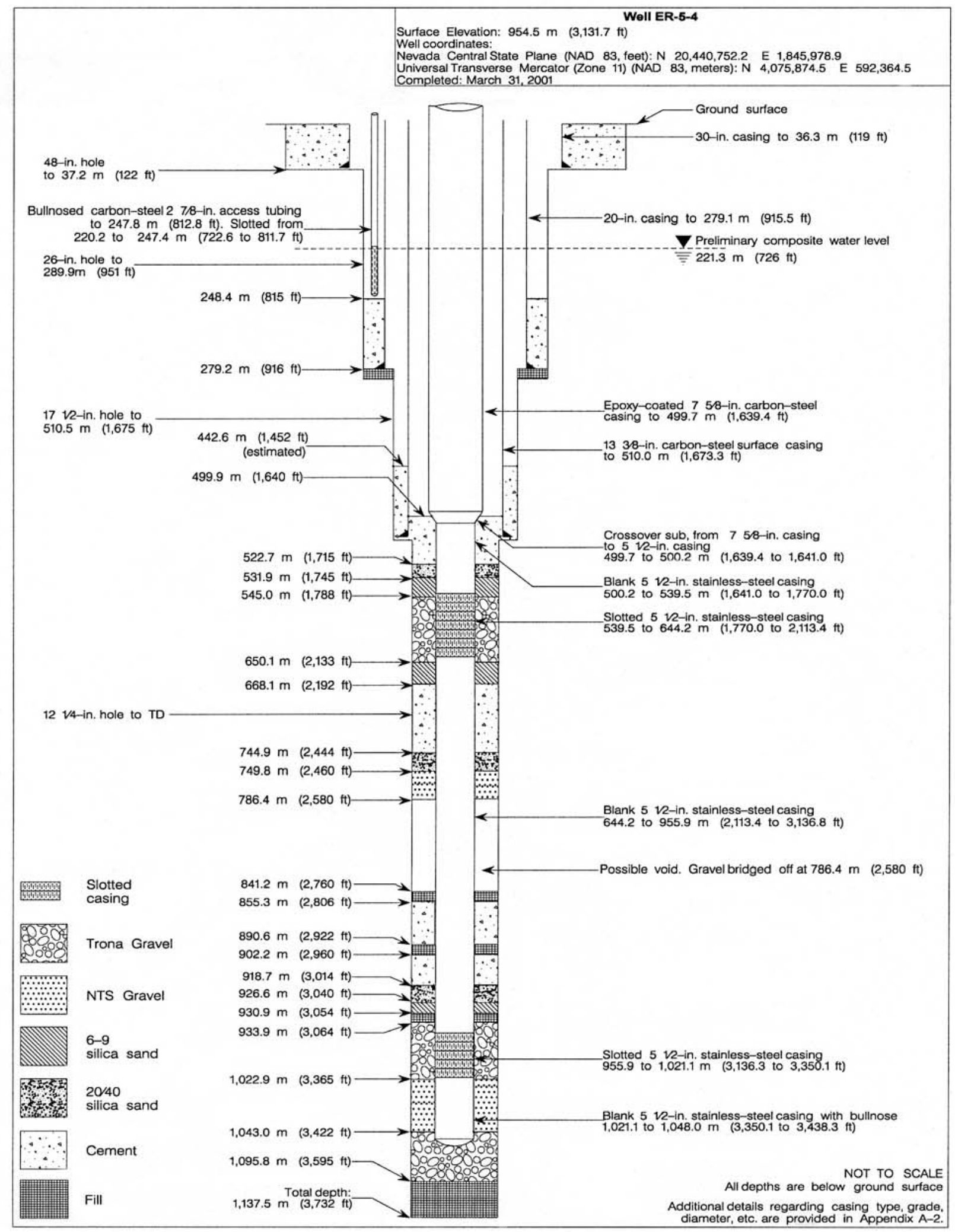

Figure 2-2

As-Built Completion Schematic for Well ER-5-4 


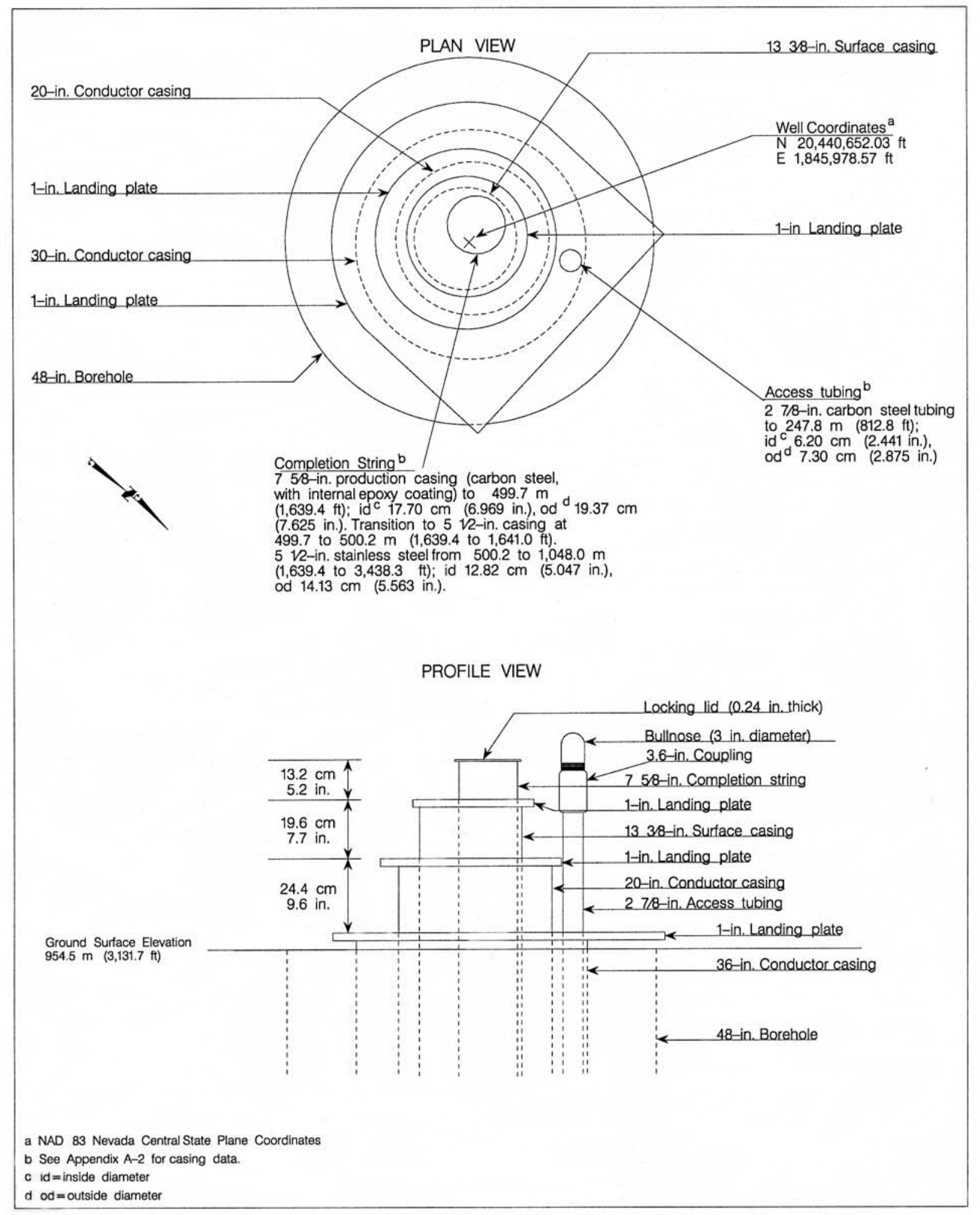

Figure 2-3

Wellhead Diagram for Well ER-5-4 
Table 2-5

Completion String Construction Summary for Well ER-5-4

\begin{tabular}{|c|c|c|c|c|}
\hline $\begin{array}{c}\text { Casing and } \\
\text { Tubing Types } \\
\end{array}$ & \multicolumn{2}{|c|}{$\begin{array}{c}\text { Configuration } \\
\text { meters (feet) }\end{array}$} & Cement & Sand/Gravel \\
\hline \multirow{3}{*}{$\begin{array}{l}2 f \text {-in. stainless-steel } \\
\text { tubing (piezometer) }\end{array}$} & \multirow{3}{*}{$\begin{array}{c}0 \text { to } 247.7 \\
\text { (0 to } 812.8)\end{array}$} & Blank & \multirow{3}{*}{ None } & \multirow{3}{*}{ None } \\
\hline & & $\begin{array}{c}\text { Slotted joints } \\
220.2 \text { to } 247.4 \\
(722.6 \text { to } 811.7) \\
\end{array}$ & & \\
\hline & & $\begin{array}{c}\text { Blank and bull-nosed } \\
247.4 \text { to } 247.7 \\
\text { (811.7 to } 812.8)\end{array}$ & & \\
\hline $\begin{array}{l}7 e \text {-in. carbon-steel } \\
\text { production casing with } \\
\text { internal epoxy coating }\end{array}$ & $\begin{array}{c}0 \text { to } 499.7 \\
(0 \text { to } 1,639.4)\end{array}$ & \multirow[b]{2}{*}{ Blank } & None & \multirow[b]{2}{*}{ None } \\
\hline $\begin{array}{l}7 \text { e -in. to } 51 / 2 \text {-in. cross- } \\
\text { over sub, carbon-steel, } \\
\text { with stainless-steel } \\
\text { double pin }\end{array}$ & $\begin{array}{c}499.7 \text { to } 500.2 \\
(1,639.4 \text { to } 1,641.0)\end{array}$ & & $\begin{array}{c}\text { Type II } \\
499.9 \text { to } 500.2 \\
(1,640 \text { to } 1,641)\end{array}$ & \\
\hline \multirow{6}{*}{$\begin{array}{l}51 / 2 \text {-in. stainless-steel } \\
\text { production casing }\end{array}$} & \multirow{6}{*}{$\begin{array}{c}500.2 \text { to } 1,048.0 \\
(1,641.0 \text { to } 3,438.3)\end{array}$} & $\begin{array}{c}\text { Blank } \\
500.2 \text { to } 539.5 \\
(1,641.0 \text { to } 1,770.0)\end{array}$ & \multirow{2}{*}{$\begin{array}{l}\text { Type II } \\
500.2 \text { to } 522.7 \\
(1,641 \text { to } 1,715)\end{array}$} & $\begin{array}{c}\frac{20 / 40 \text { Sand }}{522.7 \text { to } 531.9} \\
(1,715 \text { to } 1,745) \\
744.9 \text { to } 749.8 \\
(2,444 \text { to } 2,460) \\
918.7 \text { to } 926.6\end{array}$ \\
\hline & & $\begin{array}{c}8 \text { consecutive } \\
\text { slotted joints } \\
539.5 \text { to } 644.2 \\
(1,770.0 \text { to } 2,113.4)\end{array}$ & & $\begin{array}{l}(3,014 \text { to } 3,040) \\
\frac{6-9 \text { Sand }}{531.9 \text { to } 545.0} \\
(1,745 \text { to } 1,788)\end{array}$ \\
\hline & & & $\begin{array}{c}\text { Type II } \\
668.1 \text { to } 744.9 \\
(2,192 \text { to } 2,444)\end{array}$ & \multirow{2}{*}{$\begin{array}{c}650.1 \text { to } 668.1 \\
(2,133 \text { to } 2,192) \\
\\
926.6 \text { to } 930.9 \\
(3,040 \text { to } 3,054) \\
\frac{3 / 8 \text {-in. } \times 4 \text { Gravel }}{545.0 \text { to } 650.1} \\
(1,788 \text { to } 2,133)\end{array}$} \\
\hline & & $\begin{array}{c}\text { Blank } \\
644.1 \text { to } 955.9 \\
(2,113.4 \text { to } 3,136.3)\end{array}$ & \multirow{3}{*}{$\begin{array}{c}\text { Fill } \\
890.6 \text { to } 902.2 \\
(2,922 \text { to } 2,960)\end{array}$} & \\
\hline & & $\begin{array}{c}5 \text { consecutive } \\
\text { slotted joints } \\
955.9 \text { to } 1,021.1 \\
(3,136.3 \text { to } 3,350.1)\end{array}$ & & $\begin{array}{c}\frac{\text { Possible Void }}{\text { b }}^{\text {b }}{ }^{2,46.4 \text { to } 841.2} \\
(2,580 \text { to } 2,760) \\
841.2 \text { to } 855.3\end{array}$ \\
\hline & & $\begin{array}{c}\text { Blank and bull-nosed } \\
1,021.1 \text { to } 1,048.0 \\
(3,350.1 \text { to } 3,438.3)\end{array}$ & & $\begin{array}{r}930.9 \text { to } 933.9 \\
3,054 \text { to } 3,064) \\
\\
\begin{array}{c}3 / 8 \text {-in. } \times 4 \text { Gravel } \\
933.9 \text { to } 1,095.8 \\
(3,064 \text { to } 3,595)\end{array} \\
\end{array}$ \\
\hline
\end{tabular}

a Trona gravel except 2 sections of NTS gravel not adjacent to slotted intervals, at 749.8 to $786.4 \mathrm{~m} \mathrm{(2,460} \mathrm{to}$ $2,580 \mathrm{ft})$ and $1,022.9$ to $1,043.0 \mathrm{~m}(3,356$ to $3,422 \mathrm{ft})$.

b Gravel bridge at $786.4 \mathrm{~m}(2,580 \mathrm{ft})$. 


\subsubsection{As-Built Completion Design}

The design of the Well ER-5-4 completion was determined through consultation with members of the UGTA TWG, on the basis of onsite evaluation of data such as lithology and water production, drilling data (lost circulation, etc.), and data from various geophysical logs.

The as-built completion design for Well 5-4 provides access to the alluvial aquifer at 3 depths (Figure 2-2). The composition of the string summarized here is detailed on Table 2-5, and the casing materials are listed in Appendix A-2. The lower section of the completion string, from 500.2 to $1,048.0 \mathrm{~m}$ (1,641.0 to 3,438.3 ft), is stainless-steel $5 \frac{1}{2}-$-in. casing. The top of the $5 \frac{1}{2}-$-in. casing is approximately $279 \mathrm{~m}(915 \mathrm{ft})$ below the static fluid level. The bottom $26.9 \mathrm{~m}(88.2 \mathrm{ft})$ of the string is blank and terminates in a bull-nose to serve as a sediment sump. Above the 51/2-in. casing, a 0.49-m (1.6-ft) long cross-over sub serves as the transition to the upper part of the string, which is $7 \mathrm{e}$-in. carbon-steel production casing with an internal epoxy coating. A bull-nosed piezometer string of stainless-steel $2 f$-in. tubing is set in the annulus of the 20-in. casing at a depth of $247.7 \mathrm{~m}$ (812.8 ft).

The lowest slotted interval, 955.9 to $1,021.1 \mathrm{~m}$ ( $3,136.3$ to $3,350.1 \mathrm{ft})$, is open to sandy, Tertiary-age alluvium. This casing interval consists of 5 consecutive slotted joints. The upper slotted interval of the 51/2-in. casing, 539.5 to $644.2 \mathrm{~m}$ (1,770.0 to 2,113.4 ft), consists of 8 consecutive slotted joints. It is open to sandy Quaternary or Tertiary alluvium. The piezometer string placed between the borehole wall and the 20-in. casing contains 3 consecutive slotted joints in the depth interval 220.2 to $247.4 \mathrm{~m}$ ( 722.6 to $811.7 \mathrm{ft}$ ). This completion zone is open to the upper alluvial aquifer at the top of the saturated zone and is not gravel packed (the annulus is open).

The openings in each slotted casing joint are $0.198 \mathrm{~cm}$ ( 0.078 in.) wide and 6.35 to $7.62 \mathrm{~cm}$ ( 2.5 to 3.0 in.) long, cut in rings of 18 slots (spaced 20 degrees apart around the joint). The rings are spaced $15.2 \mathrm{~cm}(6 \mathrm{in}$.) apart, and the longitudinal centers of the slots in each ring are staggered 10 degrees from the slot centers in the next ring. No slots are cut within $0.6 \mathrm{~m}(2 \mathrm{ft})$ of the ends of the slotted joints to assure that the strength of the pipe near the connections is not degraded.

The openings in the $2 f$-in. piezometer tube are $5.715 \mathrm{~cm}$ ( $2.25 \mathrm{in}$.) long, and $0.030 \mathrm{~cm}(0.012 \mathrm{in}$.) wide, placed on staggered 15.2-cm (6-in.) centers. 


\subsubsection{Rationale for Differences between Actual and Proposed Well Design}

The proposed well design was based on the expectation that the Well ER-5-4 borehole would penetrate the entire alluvial section and enter the underlying volcanic tuffs. The original plan to construct 3 completions in the lower, middle, and upper saturated alluvial sections was accomplished.

\subsubsection{Well Completion Method}

A "tremie" line and the completion string were landed after a brief period of circulation and conditioning of the hole. The 2 completion zones of the 51/2-in. string were gravel-packed and isolated from each other with sand and cement barriers. One additional gravel layer (instead of cement) was placed adjacent to a blank interval (Figure 2-2; Table 2-5) to save time waiting for cement deliveries. Caliper logs were used to calculate the volumes of stemming materials needed during well completion. Well-construction materials were inspected according to relevant procedures; standard decontamination procedures were employed to prevent the introduction of contaminants into the well.

The filter pack around each open interval consists of $0.95-\mathrm{cm}(\mathrm{d}$-in.) by 4-mesh washed pea gravel or "Trona" gravel. However, due to lack of availability of enough Trona gravel, gravel from the NTS batch plant was substituted in some of the blank intervals (see Table 2-5 and following paragraph). The gravelpacked sections of the lower 2 completion zones are topped with 6-9 Colorado silica sand directly above the gravel, and 20/40 sand on top of the 6-9 sand. In this stemming design, developed by the UGTA program at the NTS, the layer of 20/40 sand serves as a barrier to any fluids that might seep from the cement above, preventing cement fluids from contaminating the groundwater (fluids from the cement would have the effect of drastically raising the $\mathrm{pH}$ of the groundwater). The underlying layer of 6-9 sand prevents the 20/40 sand from infiltrating the gravel-packed interval. All cement used in stemming the completion string was type II Portland cement with no additives. A clear-water pre-flush and back-flush were made at each stage of emplacement. Gravel, sand, and cement were emplaced through a $2 f$-in. Hydril ${ }^{\circledR}$ tremie line that was withdrawn as the completion process progressed. A nuclear annulus investigation log ("NAIL log") was used to monitor the emplacement of stemming materials. As-built positions of the well materials are shown on Figure 2-2 and listed in Table 2-5.

Stemming of the hole began with the first stage of Trona gravel emplaced from 1,095.8 to 1,043.0 m (3,595 to 3,422 ft) on top of $41.8 \mathrm{~m}$ ( $137 \mathrm{ft}$ ) of fill at the bottom of the hole. This layer of gravel is topped by a $20.1-\mathrm{m}$ ( $66-\mathrm{ft})$ interval of NTS gravel, to the depth of 1,022.9 $\mathrm{m}(3,356 \mathrm{ft})$. Trona gravel was placed above the NTS gravel, adjacent to the lowest slotted interval. This gravel pack is topped by a $3-\mathrm{m}$ (10-ft) interval of fill which sloughed from the borehole wall before sand could be added. The sand barrier 
extends from the top of the fill at $930.9 \mathrm{~m}(3,054 \mathrm{ft})$ to the depth of $918.7 \mathrm{~m}(3,014 \mathrm{ft})$, and is followed by cement, poured in 2 stages up to $855.3 \mathrm{~m}(2,806 \mathrm{ft})$. Between these 2 stages of cement, the NAIL log indicates that the borehole again sloughed, creating another layer of fill between 902.2 and $890.6 \mathrm{~m}$ (2,960 and 2,922 ft). The NAIL log indicates that after the second stage of cement had been poured, $14.0 \mathrm{~m}$ (46 ft) of fill was present on top of the cement. Stemming continued with a second layer of NTS gravel, but during emplacement the gravel "bridged off" in the hole, leaving a void space adjacent to the blank $51 / 2$-in. casing, between the top of the fill at $841.2 \mathrm{~m}(2,760 \mathrm{ft})$ and $786.4 \mathrm{~m}(2,580 \mathrm{ft})$. The borehole annulus is filled with gravel above this void to the depth of $749.8 \mathrm{~m}(2,460 \mathrm{ft})$. This gravel was topped with 20/40 sand to the depth of $744.9 \mathrm{~m}$ (2,444 ft), and cement, poured in 2 stages, to the depth of $668.1 \mathrm{~m}(2,192 \mathrm{ft})$. An interval of 6-9 sand was emplaced above the cement to a depth of $650.1 \mathrm{~m}$ $(2,133 \mathrm{ft})$. The uppermost gravel section, located adjacent to the upper slotted interval of the $5 \frac{1}{2}$-in. casing, consists of Trona gravel, between the depths of 650.1 and $545.0 \mathrm{~m}(2,133$ and 1,788 ft). This gravel pack is capped with sand to a depth of $522.7 \mathrm{~m}(1,715 \mathrm{ft})$. The final cemented section, poured in 3 stages, extends to the depth of $499.9 \mathrm{~m}(1,640 \mathrm{ft})$. The upper part of the hole containing the piezometer string was left open.

The drill rig was released after cementing was completed. Hydrologic testing was planned as a separate effort, so a pump was not installed in the well and no well development or pumping tests were conducted immediately after completion.

\subsection{Actual versus Planned Costs and Scheduling}

The original BN cost model developed for Well ER-5-4 was based on drilling to the planned TD of $1,066.8 \mathrm{~m}(3,500 \mathrm{ft})$. The drilling program baseline projected that it would require 30 days to drill and complete the well. However, the actual conditions encountered during drilling of the well (severe borehole instability; deeper than planned TD) were measurably different from predicted conditions so the baseline was changed during drilling.

The new cost model is based on the actual TD of 1,137.5 $\mathrm{m}(3,732 \mathrm{ft})$. It took 50 days to accomplish drilling of the surface and main holes, logging, and completion of the well, after construction of the conductor hole by BN. Drilling of the surface hole and installation of the 20 -in. surface casing proceeded as expected. However, drilling of the production hole and installation of the completion casing took longer than predicted, and required installation of an intermediate casing string. A graphical comparison, by day, of planned and actual well-construction activities is presented in Figure 2-4. 
The cost analysis for Well ER-5-4 begins with the movement of the UDI drill rig to the Well ER-5-4 site from the site of Well Cluster ER-5-3. The cost of building roads, the drill pad, and sumps is not included, and the cost of well-site support by IT is not included. The total construction cost for Well ER-5-4 includes all drilling costs: charges by the drilling subcontractor; charges by other support subcontractors (including compressor services, drilling fluids, bits, casing services, down-hole tools, and geophysical logging); and charges by $\mathrm{BN}$ for mobilization and demobilization of equipment, construction of the conductor hole, cementing services, completion materials, radiation technicians, inspection services, and geotechnical consultation.

The total planned cost for constructing Well ER-5-4, based on the new baseline developed because the actual conditions differed greatly from the expected conditions, was $\$ 3,286,909$. The actual cost was $\$ 2,973,290$, or 9.5 percent less than the planned cost. Figure 2-5 presents a comparison of the planned (new baseline) and actual costs, by day, for drilling and completing Well ER-5-4.

\subsection{Summary, Recommendations, and Lessons Learned}

\subsubsection{Summary for Well ER-5-4}

Subcontractor activities at Well Cluster ER-5-4 commenced on February 7, 2001, with the drilling of Well ER-5-4. Drilling of Well ER-5-4 concluded on March 18, 2001, when the TD of 1,137.5 m $(3,732 \mathrm{ft})$ was reached. After geophysical logging, the completion string and piezometer were installed and the well was gravel-packed and stemmed to the depth of $499.9 \mathrm{~m}$ (1,640 ft) on March 27-31, 2001. Crews worked on a 7-days-per-week, 24-hours-per-day schedule for most of the operation. Fifty working days were expended to drill the surface and main holes, conduct geophysical logging, and install the completion string. Severe sloughing of the borehole wall during drilling, geophysical logging, and wellinstallation operations prolonged the construction of Well ER-5-4. 


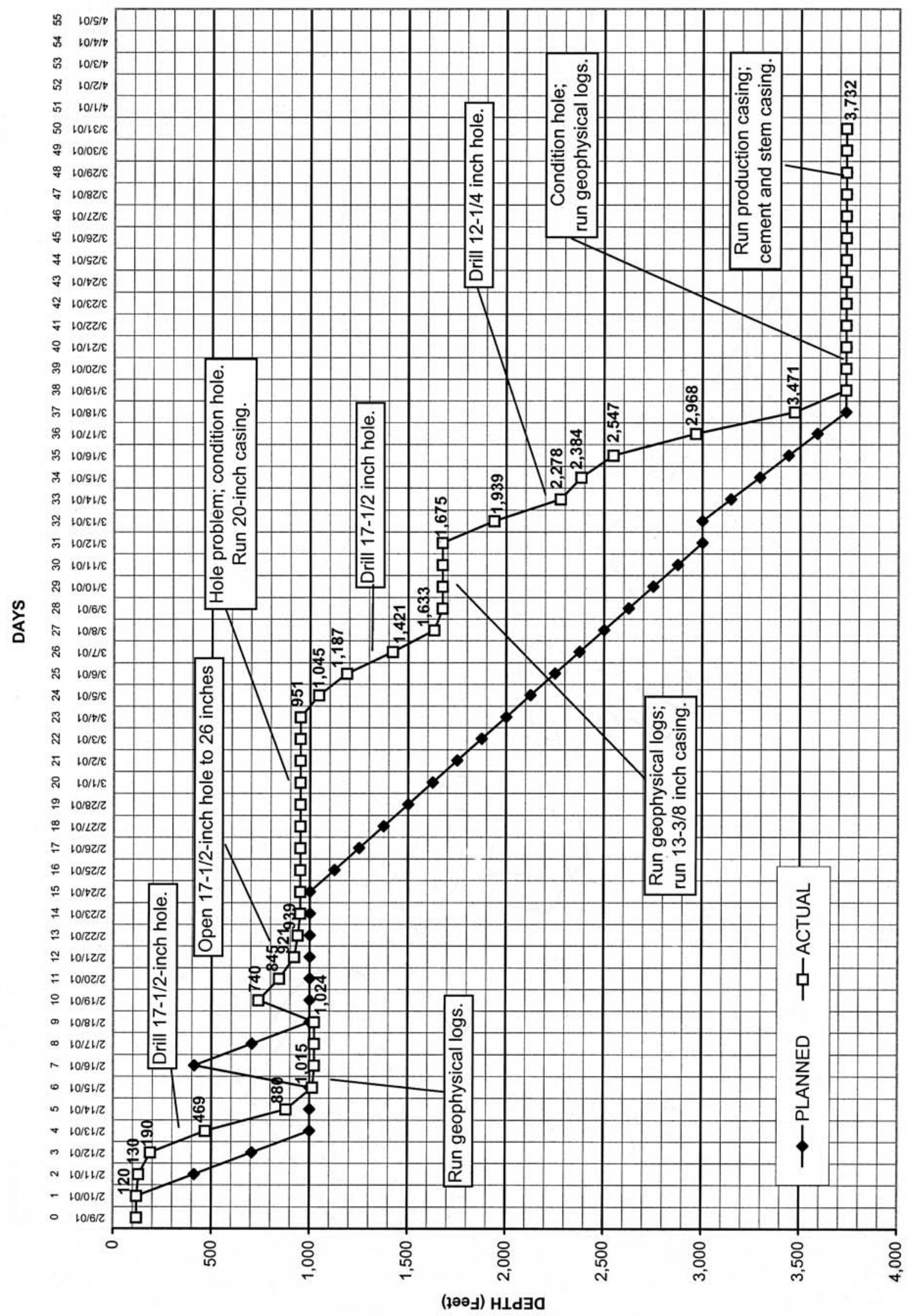

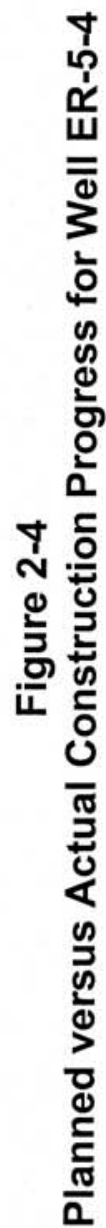




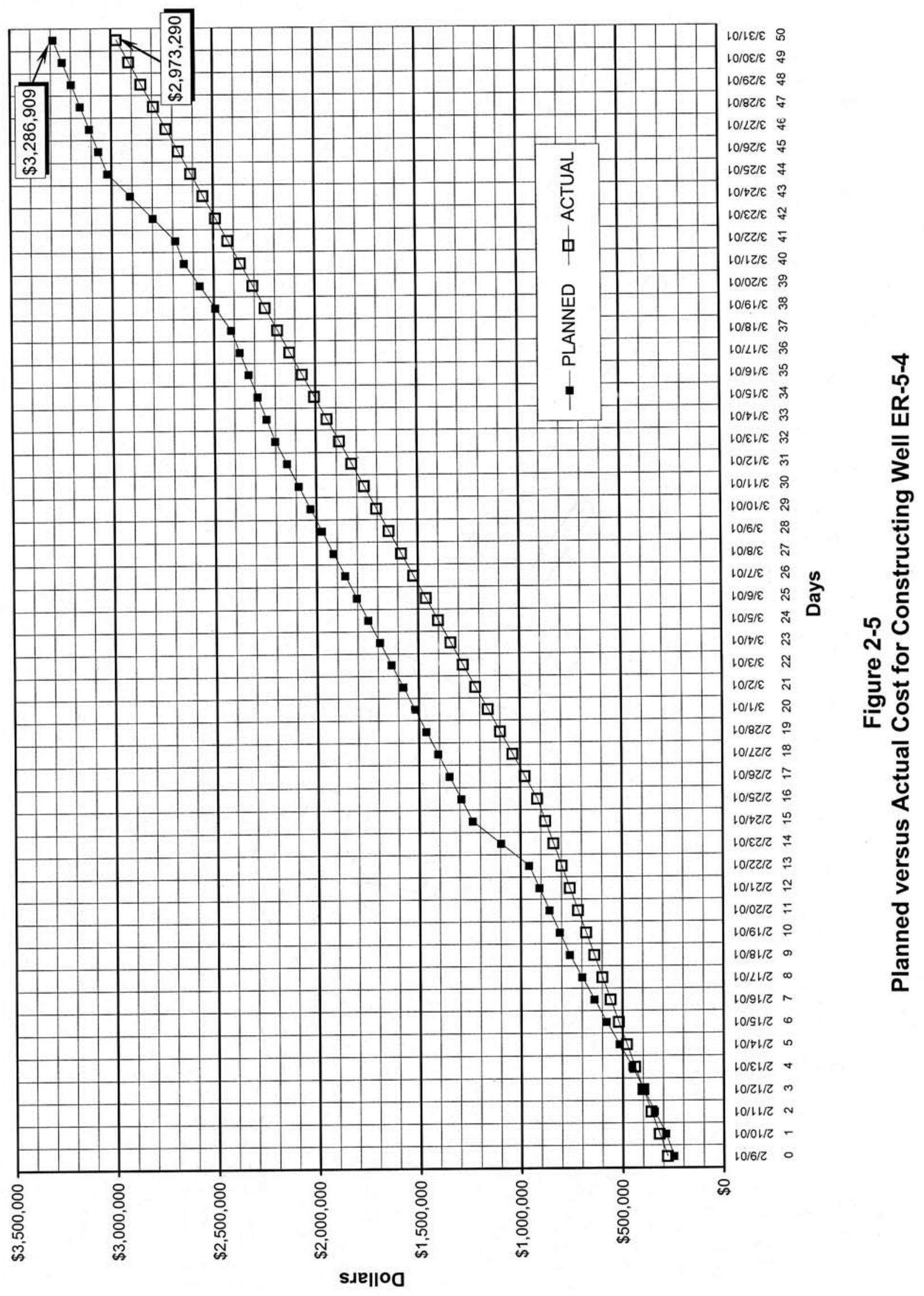


No radionuclides above background were encountered in the groundwater produced during drilling of Well ER-5-4, except in the interval 274.3 to $312.4 \mathrm{~m}$ (900 to 1,025 ft), where slightly elevated tritium levels up to $5,028 \mathrm{pCi} / \mathrm{L}$ were measured. Preliminary (field-monitoring) data indicated no lead above permitted levels for dissolved lead in the drilling effluent.

A completion string with 2 gravel-packed, slotted intervals was installed in the lower portion of Well ER-5-4, and a piezometer string was installed in the upper, open portion of the hole. A string of $51 / 2$-in. stainless-steel casing installed below the water table is suspended from $7 e$-in. carbon-steel casing that extends to the surface. The open intervals in the $5 \frac{1}{2}$-in. casing are centered within the gravel-packed intervals that are located at 933.0 to $1,095.8 \mathrm{~m} \mathrm{(3,054} \mathrm{to} \mathrm{3,595} \mathrm{ft)} \mathrm{and} 545.0$ to $650.1 \mathrm{~m}(1,788$ to 2,133 ft). The stainless-steel, $2 \mathrm{f}$-in. piezometer tube installed in the annulus of the 20 -in. casing is open to the formation (saturated alluvium). A preliminary fluid depth was measured at $221.3 \mathrm{~m}$ (726.6 ft) before the addition of bentonite mud.

\subsubsection{Recommendations}

The planned pump installation, well development, groundwater sampling, and hydrologic testing must be conducted at Well ER-5-4 to accomplish the remaining objectives for this well-construction effort. In addition, after Well ER-5-4\#2 is drilled, geologic, and hydrologic data must be evaluated and interpretations of the area hydrogeology updated and inserted into the UGTA hydrologic model. This process, in conjunction with interpretation of the 3-D seismic reflection survey conducted in this area, and followed by analysis of the updated model, will allow more precise characterization of groundwater flow direction and velocity in the Frenchman Flat CAU.

\subsubsection{Lessons Learned from Well ER-5-4}

Significant problems associated with borehole instability can occur while drilling alluvium in the central portions of Frenchman Flat. Severe sloughing and erosion of the borehole wall can occur even when drilling with bentonite mud, especially below the water table. The complete loss of circulation fluid, which is typically associated with fractured intervals in harder and more brittle units, can also occur while drilling alluvium in the central portions of Frenchman Flat. Additional casing strings and the use of cement plugs may be necessary to successfully drill through the alluvial section in Frenchman Flat. 


\subsection{Well ER-5-4\#2}

\subsection{Well-Specific Objectives}

The scientific objectives for Well ER-5-4\#2 include those listed in Section 1.2. However, the specific goal of Well ER-5-4\#2 was to penetrate through the volcanic rock section and into the underlying Paleozoicage sedimentary rocks that at this location are thought to comprise the lower carbonate aquifer. Two completion zones were to be installed, one in the middle of the volcanic rock section which was expected to be a tuff confining unit, and the other in the upper part of the lower carbonate aquifer. The planned TD of the well was $1,889.8 \mathrm{~m}(6,200 \mathrm{ft})$, which was expected to place the bottom of the well approximately $61.0 \mathrm{~m}(200 \mathrm{ft})$ into the lower carbonate aquifer.

\subsection{Drilling Summary}

This section contains detailed description of the drilling process and fluid management issues.

\subsubsection{Introduction}

The general drilling requirements for Well ER-5-4\#2 were provided in Frenchman Flat Hydrogeologic Investigation Wells Drilling and Completion Criteria (IT, 2000) and an addendum to that document (IT, 2001a). Specific requirements for Well ER-5-4\#2 were outlined in Field Activity Work Plan Number D-006-001.02 (BN, 2002). A summary of drilling statistics for the well is given in Table 3-1. Figure 3-1 is a chart of the drilling and completion history for Well ER-5-4\#2. The following information was compiled primarily from $\mathrm{BN}$ daily drilling reports.

\subsubsection{Drilling History}

Field operations at Well ER-5-4\#2 began on January 25, 2001, when a BN crew began dry-augering a 121.9-cm (48-in.) diameter conductor hole. The conductor hole was completed on the next day to a depth of $35.1 \mathrm{~m}$ (115 ft), and on January 30, 2001, a string of 30-in. conductor casing was set at $33.8 \mathrm{~m}$ (111 ft). The bottom of the casing was cemented into place and the annulus of the conductor casing was cemented to ground level with neat type II cement. A hiatus of 17 months occurred between the construction of the conductor hole by $\mathrm{BN}$ and the beginning of work on the main hole by the drilling subcontractor, UDI. 


\section{Table 3-1 \\ Abridged Drill Hole Statistics for Well ER-5-4\#2}

\begin{tabular}{|c|c|c|c|}
\hline Coordinates: & Nevada State Plane (central zone): & $\begin{array}{l}\text { NAD 83: } N \text { 6,230,323.2 m } \\
\text { NAD 27: } N 755,651.2 \mathrm{ft}\end{array}$ & $\begin{array}{l}\text { E } 562,655.4 \mathrm{~m} \\
\text { E 705,819.6 ft }\end{array}$ \\
\hline Surface Elevation ${ }^{\mathrm{a}}$ : & $\begin{array}{l}\text { Universal Transverse Mercator: } \\
954.5 \mathrm{~m}(3,131.7 \mathrm{ft})\end{array}$ & NAD 83: N 4,075,844.0 m & E 592,364.5 m \\
\hline \multirow{5}{*}{$\begin{array}{l}\text { DRILLING DATA: } \\
\text { Spud Date: } \\
\text { Total Depth (TD): } \\
\text { Date TD Reached: } \\
\text { Date Well Completed }{ }^{\mathrm{b}} \text { : } \\
\text { Hole Diameter: }\end{array}$} & \multirow{2}{*}{\multicolumn{3}{|c|}{ 07/16/2002 (main hole drilling with Wilson Mogul 42B rig) }} \\
\hline & & & \\
\hline & \multirow{2}{*}{\multicolumn{3}{|c|}{$\begin{array}{l}09 / 11 / 2002 \\
09 / 18 / 2002\end{array}$}} \\
\hline & & & \\
\hline & \multicolumn{3}{|c|}{$\begin{array}{l}121.9 \mathrm{~cm}(48 \mathrm{in} .) \text { from surface to } 35.1 \mathrm{~m}(115 \mathrm{ft} .) ; 66.0 \mathrm{~cm}(26 \mathrm{in} .) \text { from } 35.1 \mathrm{~m}(115 \mathrm{ft} .) \text { to } \\
311.8 \mathrm{~m}(1,023 \mathrm{ft} .) ; 44.5 \mathrm{~cm}(17.5 \mathrm{in} .) \text { from } 311.8 \mathrm{~m}(1,023 \mathrm{ft} .) \text { to } 1,052.8 \mathrm{~m}(3,454 \mathrm{ft}) ; \\
31.1 \mathrm{~cm}(12.25 \mathrm{in} .) \text { from } 1,052.8 \mathrm{~m}(3,454 \mathrm{ft}) \text { to } 1,573.7 \mathrm{~m}(5,163 \mathrm{ft}) ; 22.2 \mathrm{~cm}(8.75 \mathrm{in} .) \\
\text { from } 1,573.7 \mathrm{~m}(5,163 \mathrm{ft}) \text { to } 2,133.6 \mathrm{~m}(7,000 \mathrm{ft}) .\end{array}$} \\
\hline Drilling Techniques: & \multicolumn{3}{|c|}{ 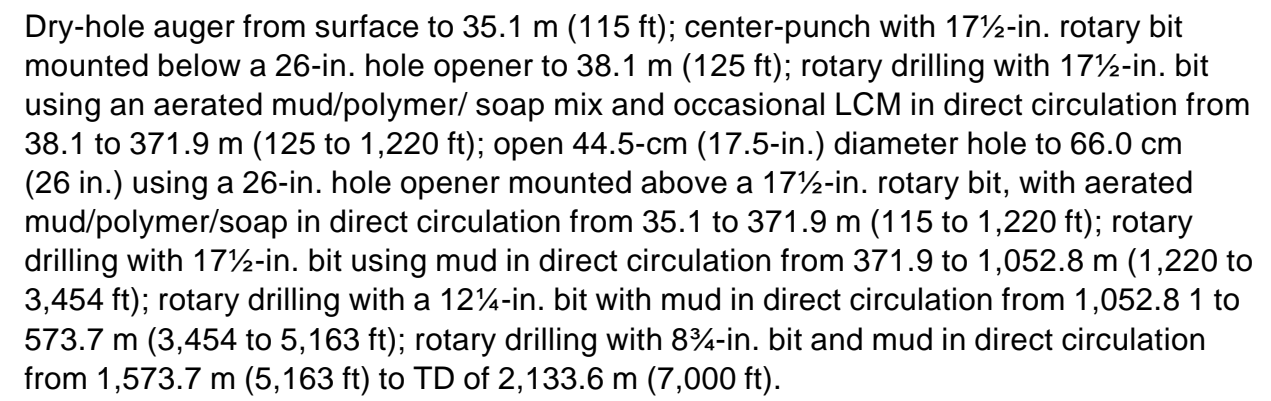 } \\
\hline \multicolumn{4}{|c|}{$\begin{array}{ll}\text { CASING DATA: } & \text { 30-in. conductor casing from surface to } 33.8 \mathrm{~m}(111 \mathrm{ft}) ; 20 \text {-in. surface casing from surface to } \\
& 266.1 \mathrm{~m}(873.0 \mathrm{ft}) ; 13 \mathrm{~d} \text {-in. intermediate casing from surface to } 965.9 \mathrm{~m}(3,169.0 \mathrm{ft}) ; 9 \mathrm{e} \text {-in. } \\
& \text { intermediate casing from surface to } 1,477.9 \mathrm{~m}(4,848.8 \mathrm{ft}) .\end{array}$} \\
\hline \multicolumn{4}{|c|}{$\begin{array}{l}\text { WELL COMPLETION DATA: } \\
\text { The completion string consists of approximately } 592.8 \mathrm{~m}(1,945 \mathrm{ft}) \text { of } 14.1-\mathrm{cm}(51 / 2-\mathrm{in} .) \text { stainless steel casing } \\
\text { hung from within the } 24.4-\mathrm{cm}(9 \mathrm{e}-\mathrm{in} .) \text { intermediate casing using a liner hanger positioned at } 1,437.1 \mathrm{~m} \\
(4,715 \mathrm{ft}) \text {. The stainless steel casing has an outside diameter of } 14.13-\mathrm{cm}(5.563-\mathrm{in} .) \text { and an inside diameter } \\
\text { of } 12.8-\mathrm{cm} \text { ( } 5.047 \mathrm{in} .) \text {, is bull-nosed, and has a single slotted interval from } 1,976.9 \text { to } 2,029.3 \mathrm{~m}(6,486.0 \text { to } \\
6,657.7 \mathrm{ft}) \text { consisting of consecutive slotted joints. No sand or gravel packs were installed, and the casing } \\
\text { was not cemented. No pumps were installed at time of completion. }\end{array}$} \\
\hline \multicolumn{2}{|l|}{ Total Depth: } & \multicolumn{2}{|l|}{$2,030.0 \mathrm{~m}(6,660 \mathrm{ft})$} \\
\hline \multicolumn{2}{|c|}{ Depth of Slotted Sections in 51/2-in. Production Casing: } & \multicolumn{2}{|c|}{$1,976.9$ to $2,029.3 \mathrm{~m}(6,486.0$ to $6,657.7 \mathrm{ft})$} \\
\hline \multicolumn{2}{|c|}{ Depth of Sand Packs: } & \multicolumn{2}{|l|}{ None } \\
\hline \multicolumn{2}{|c|}{ Depth of Gravel Packs: } & \multicolumn{2}{|l|}{ None } \\
\hline \multicolumn{2}{|l|}{ Depth of Pump: } & \multicolumn{2}{|c|}{ Not installed at time of completion } \\
\hline \multicolumn{2}{|l|}{ Water Depth ${ }^{\mathrm{c}}$ : } & \multicolumn{2}{|l|}{$215.7 \mathrm{~m}(707.6 \mathrm{ft})$} \\
\hline \multicolumn{2}{|l|}{ DRILLING CONTRACTOR: } & & \\
\hline GEOPHYSICAL LOGS BY: & \multicolumn{3}{|c|}{ Halliburton Energy Services, Desert Research Institute, Baker Hughes Inteq } \\
\hline \multicolumn{4}{|c|}{ SURVEYING CONTRACTOR: Bechtel Nevada } \\
\hline
\end{tabular}

a Elevation of ground level at wellhead. 1929 National Geodetic Vertical Datum.

b Date completion string was cemented. Pump will be installed at a later date as needed.

c Measured on September 18, 2002 (IT, 2003). 


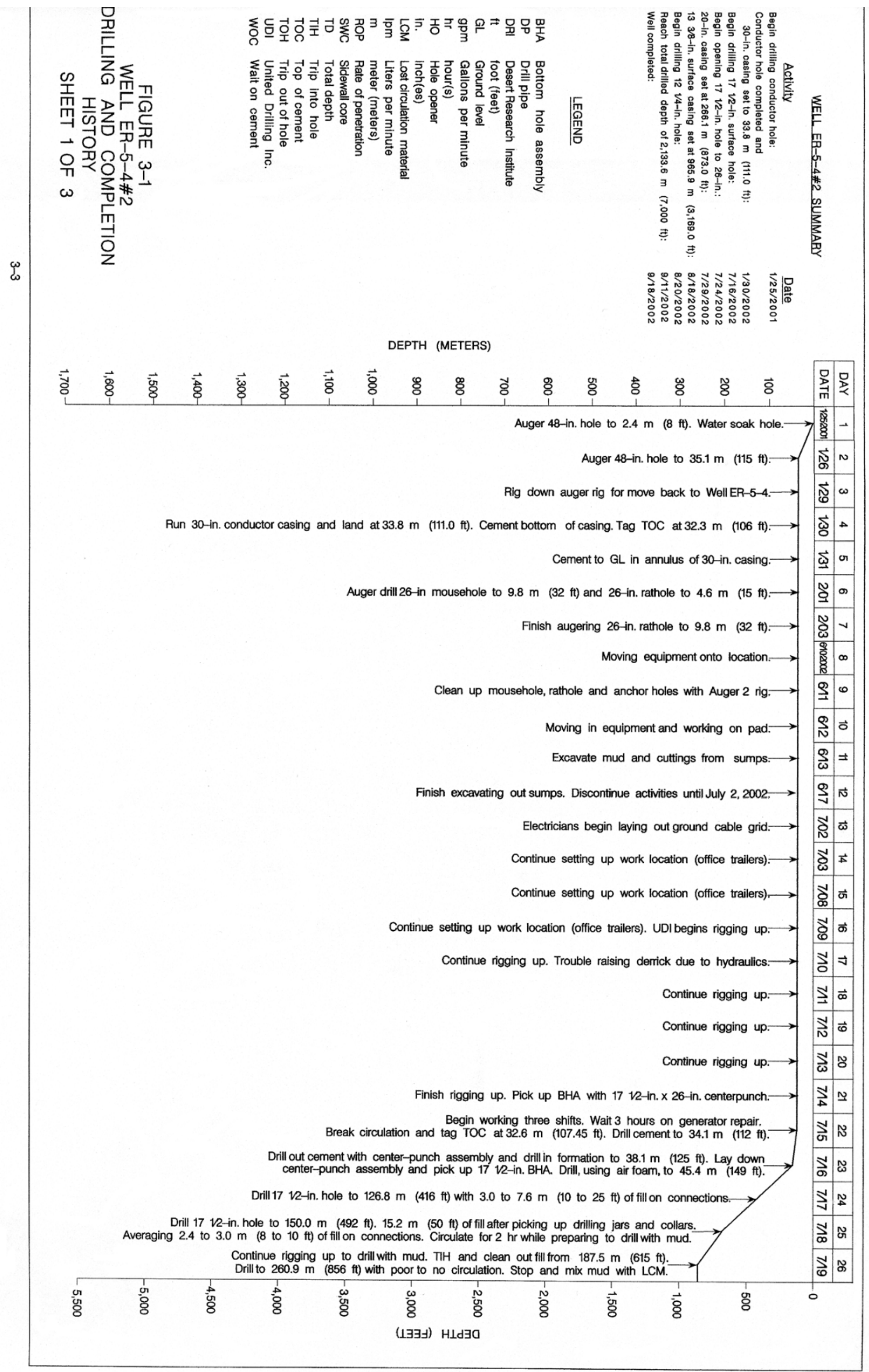




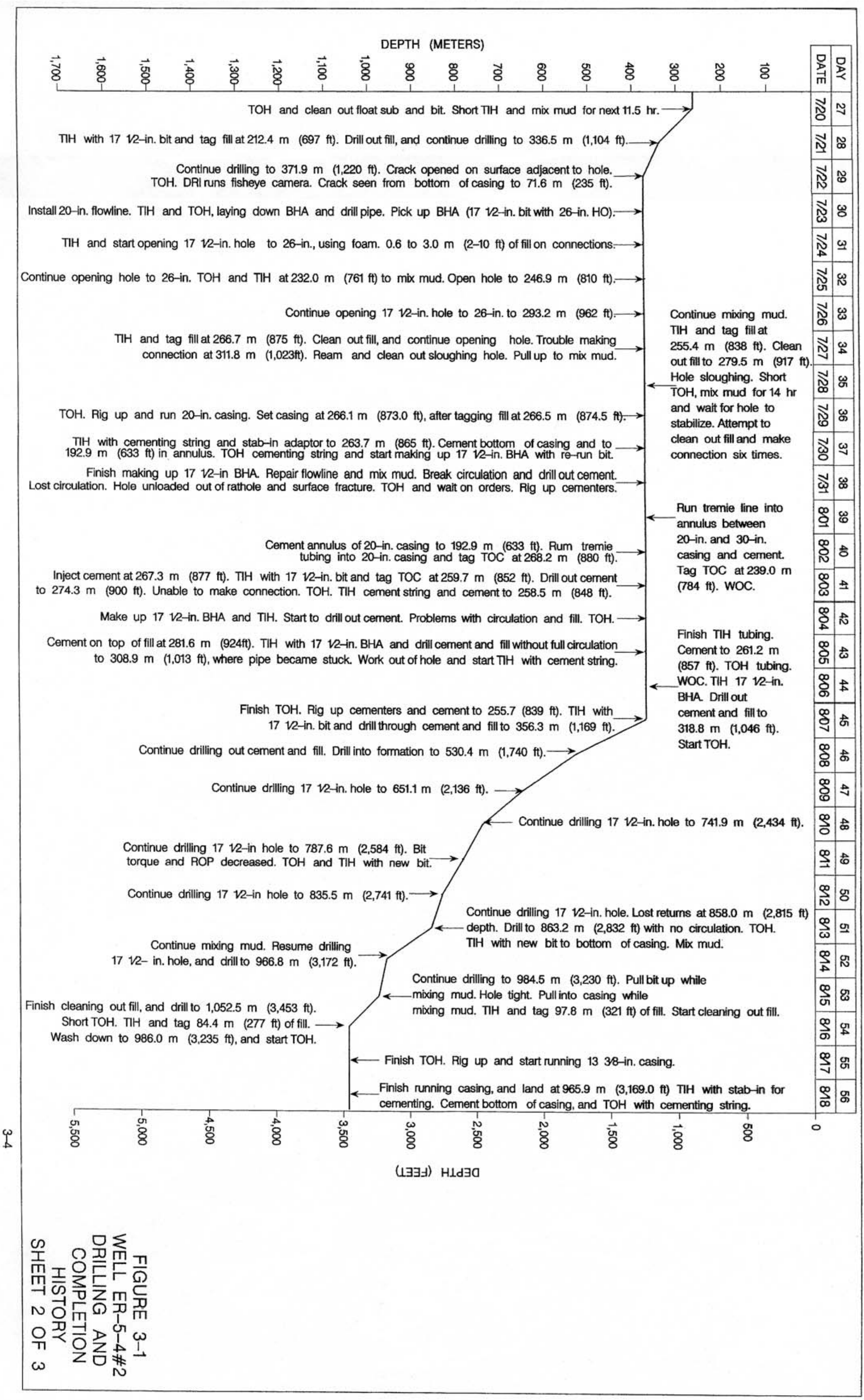




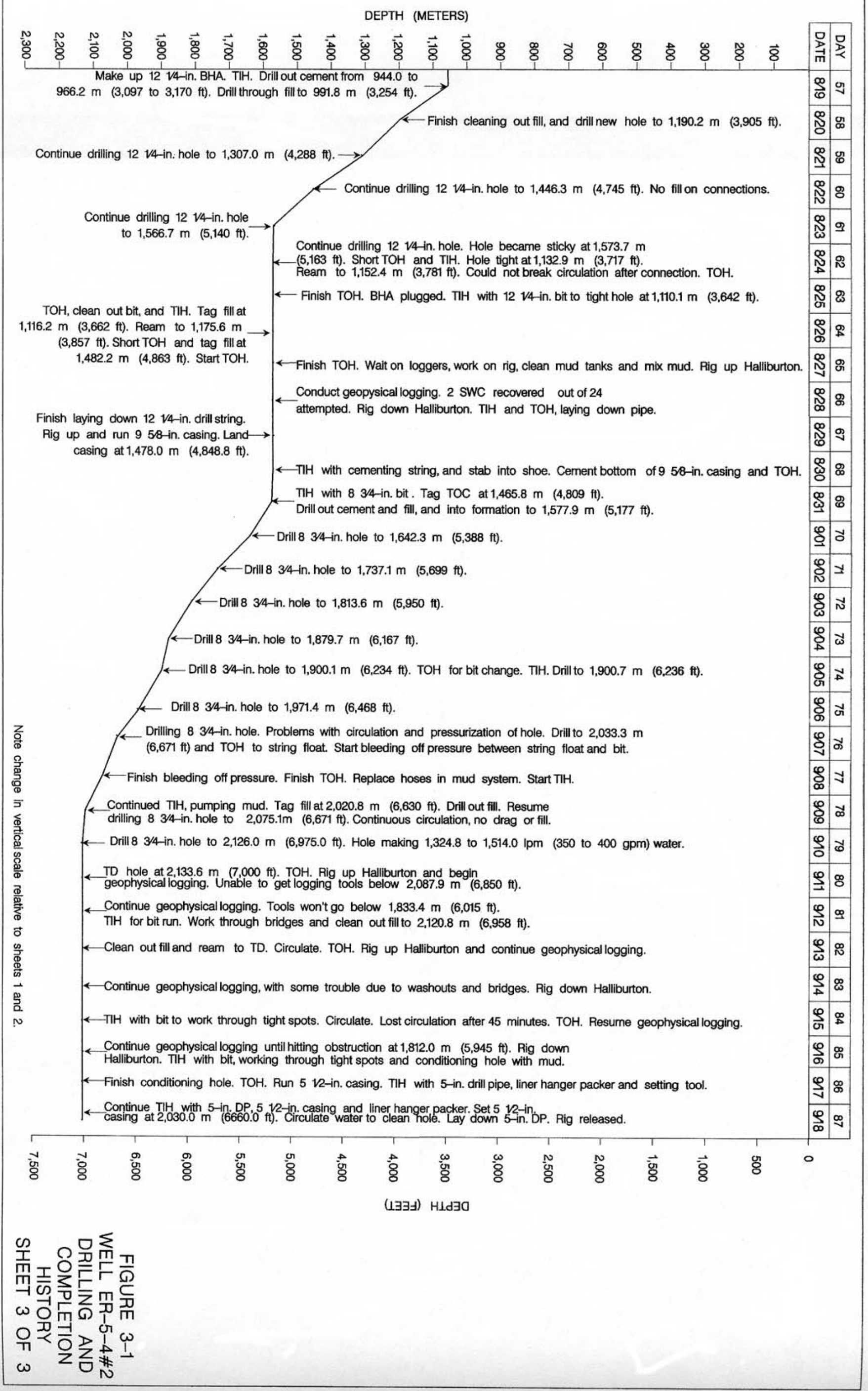


The UDI crew rigged up the Wilson Mogul 42B drill rig July 9 to 15, 2002. They tagged the top of cement in the conductor hole at $32.6 \mathrm{~m}(107 \mathrm{ft})$ with a center-punch assembly consisting of a 171/2-in. rotary bit mounted below a 26-in. hole opener. When the hole opener had cleared the bottom of the conductor casing (hole depth at $38.1 \mathrm{~m}$ [125 ft]), the center-punch assembly was replaced with a 171/2-in. BHA and drilling continued. The drilling fluid was air-foam in conventional circulation. Drilling continued to $205.4 \mathrm{~m}$ (674 ft) with 3.0 to $7.6 \mathrm{~m}(10$ to $25 \mathrm{ft})$ of fill encountered after most connections. At that depth the fluid was changed to an aerated mix of bentonite mud, polymer, and gel. This mix was expected to help minimize borehole instability, particularly near the water table (expected at the depth of approximately $221.3 \mathrm{~m}$ [726 ft]), where severe borehole sloughing had been experienced during drilling of Well ER-5-4 (Section 2.1).

When drilling resumed, loss of drill fluid to the formation prompted the addition of LCM to the mud to help

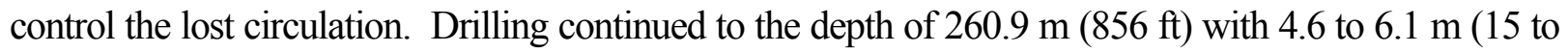
$20 \mathrm{ft}$ ) of fill on connections. Fluid returns became intermittent, and additional mud with gel, polymer, and LCM was pumped down hole in an effort to regain and maintain fluid circulation. The bit became plugged, so the drillers pulled the drill string from the hole and cleaned out the bit and float sub. No drilling was accomplished while additional mud was mixed for the next 11.5 hours, then the $171 / 2$-in. BHA was lowered back into the hole and drilling resumed to $276.8 \mathrm{~m}(908 \mathrm{ft})$, stopping occasionally to mix more mud.

Drilling had reached the depth of $371.9 \mathrm{~m}(1,220 \mathrm{ft})$ on July 22, 2002, when a crack opened at the surface with drilling fluid flowing out of it. The crack was oriented approximately north-south; it extended through the borehole and for a distance of 15.2 to $21.3 \mathrm{~m}$ (50 to $70 \mathrm{ft}$ ) each side of the hole. Drilling was halted and the drill string removed from the hole, so that a camera could be run in the borehole. The camera was run by DRI to the top of foam in the hole at $129.5 \mathrm{~m}$ (425 ft). The crack was observed to extend across the borehole from the base of the conductor casing at $33.8 \mathrm{~m}(111 \mathrm{ft})$ to a depth of $71.6 \mathrm{~m}(235 \mathrm{ft})$. The decision was made to open the hole to $66.0 \mathrm{~cm}$ (26 in.) in diameter so that a string of 20 -in. surface casing could be installed to seal off the crack and to control sloughing of the borehole.

Over the next 5 days, while attempting to open the 44.4-cm (17.5-in.) diameter hole to $66.0 \mathrm{~cm}$ (26 in.), the drillers repeatedly encountered severe sloughing of the borehole, large accumulations of fill, and loss of large quantities of drill fluid to the formation. The crew repeatedly worked their way through tight spots, reaming and cleaning the hole, and stopping for as much as 10 to 12 hours at a time to mix more drilling 
mud. Finally, these efforts were halted at the depth of $371.9 \mathrm{~m}(1,220 \mathrm{ft})$, and on July 29, 2002, the drill string was removed from the borehole in preparation for running the surface casing.

As the casing crew began running the 20-in. casing into the hole, the wireline parted and allowed some of the rigging to fall on top of the casing tongs. After a 4-hour suspension of work to re-rig the tongs and inspect the rigging, work resumed. The 20-in. casing was run in the hole and landed at $266.1 \mathrm{~m}(873.0 \mathrm{ft})$ after the top of fill was tagged at $266.5 \mathrm{~m}(874.5 \mathrm{ft})$. A string of 5-in. drill pipe with a stab-in adaptor was run inside the casing and stabbed into the casing guide shoe. Neat type II cement was pumped down the drill pipe to cement the bottom of the casing.

After the bottom of the casing was cemented, a BHA with a 171/2-in. bit was made up and lowered into the hole. The casing guide shoe and cement were drilled out to $266.1 \mathrm{~m}(873 \mathrm{ft})$, at which point circulation was lost. After reaming and cleaning out fill and trying to regain circulation, the crew made a connection at $266.1 \mathrm{~m}(873 \mathrm{ft})$, but fluid was forced from the annulus between the 20-in. and 30-in. casings, and from the rathole and the surface crack. This indicated that the bottom of the 20 -in. casing was not completely cemented, and the annular space between the 20 -in. casing and the borehole wall was not sealed off. After removing the drill pipe from the hole, drillers ran a "tremie" tubing line down the annulus between the 20-in. casing and the borehole wall so that cement could be placed directly within the annulus. The annulus was cemented through the tubing in 8 stages with neat type II cement, and it was later determined that the top of cement within the annulus was at the depth of $192.9 \mathrm{~m}(633 \mathrm{ft})$. The tremie line was then pulled out and placed inside the 20-in. casing to pump additional cement at the bottom of the casing.

When drilling resumed with the $171 / 2$-in. bit, the top of cement in the 20 -in. casing was tagged at the depth of $259.7 \mathrm{~m}$ (852 ft). Cement and fill were drilled out to a depth of $274.3 \mathrm{~m}$ (900 ft), but circulation was lost at $263.7 \mathrm{~m}(865 \mathrm{ft})$ and the connection at $274.3 \mathrm{~m}(900 \mathrm{ft})$ could not be made due to the accumulation of fill. The drill string was removed and a tremie line lowered inside the 20 -in. casing to place a cement plug at the bottom of the hole to help stabilize the borehole and reduce sloughing and lost circulation. After the cement job, the top of cement was tagged at $258.5 \mathrm{~m}(848 \mathrm{ft})$, and cement and fill were drilled out to a depth of $289.6 \mathrm{~m}$ (950 ft). Continuous sloughing and lost circulation were again encountered, necessitating the placement of additional cement in the hole. After this cement job the top of cement was tagged at $260.0 \mathrm{~m}(853 \mathrm{ft})$. Cement was drilled out to $284.1 \mathrm{~m}$ (932 ft), and fill to $308.8 \mathrm{~m}(1,013 \mathrm{ft})$ with 3.0 to $4.6 \mathrm{~m}$ (10 to $15 \mathrm{ft})$ of fill encountered on connections and circulation lost at $266.1 \mathrm{~m}(873 \mathrm{ft})$. At the depth of $308.8 \mathrm{~m}(1,013 \mathrm{ft})$ the pipe became stuck, and after the drillers worked it free they removed the drill string and cemented the bottom of the hole again, up to the depth of $261.2 \mathrm{~m}(857 \mathrm{ft})$. Cement was drilled 


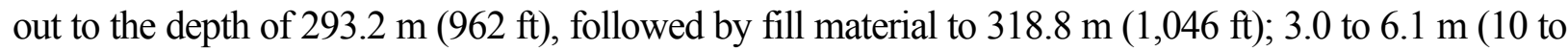
$20 \mathrm{ft}$ ) of fill was encountered on connections. An additional load of cement was placed at the bottom of the hole to a depth of $255.7 \mathrm{~m}(839 \mathrm{ft})$, and the cement and fill were again drilled out to the original depth of the 171/2-in hole at $371.9 \mathrm{~m}(1,220 \mathrm{ft})$ on August 8, 2002.

Drilling then continued more smoothly, reaching the depth of $787.6 \mathrm{~m}(2,584 \mathrm{ft})$ on August 11, 2002, at which time the drill bit was replaced with a new 171/2-in. bit. Drilling then continued to $863.2 \mathrm{~m}(2,832 \mathrm{ft})$, on August 13, 2002, when the drillers pulled the pipe from the hole and found that the drill bit had a loose cone and a missing seal. As drilling proceeded with a new 171/2-in. bit, water production increased and $6.1 \mathrm{~m}$ (20 ft) of fill was encountered at connections below $923.5 \mathrm{~m}(3,030 \mathrm{ft})$. At the depth of $984.5 \mathrm{~m}$ $(3,230 \mathrm{ft})$ tight hole conditions were encountered while pulling the drill bit off bottom to mix mud. The crew worked the drill bit through the tight spots and pulled the bit up into the 20 -in. casing while more mud was mixed. When the drill pipe was lowered again, the bit tagged the top of fill at $886.7 \mathrm{~m}(2,909 \mathrm{ft})$, indicating that $97.8 \mathrm{~m}(321 \mathrm{ft})$ of fill had accumulated. The fill was cleaned out and the $44.4-\mathrm{cm}\left(17 \frac{1}{2}-\mathrm{in}\right.$.)

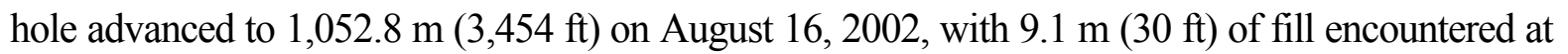
connections. The drillers added high viscosity mud to the hole to control sloughing, and pulled up the bit to

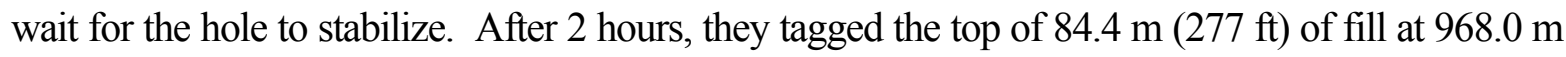
$(3,176 \mathrm{ft})$. They cleaned out the fill to $986.0 \mathrm{~m}(3,235 \mathrm{ft})$, at which point the drill pipe was pulled from the hole in preparation for installing an intermediate casing.

The $13 \mathrm{~d}$-in. intermediate casing was run in the hole by a casing subcontractor on August 17, 2002. After working through an obstruction at $285.9 \mathrm{~m}$ (938 ft), the casing tagged top of fill at $950.4 \mathrm{~m}(3,118 \mathrm{ft})$. The crew washed the casing down through the fill and landed it at the depth of $965.9 \mathrm{~m}(3,169.0 \mathrm{ft})$. A string of 5-in. drill pipe was run inside the casing and stabbed into the float shoe to cement the bottom of the casing.

On August 19, 2002, the crew made up a BHA with a 121/4-in. bit and lowered it into the hole. The top of cement was tagged at $944.0 \mathrm{~m}(3,097 \mathrm{ft})$, and $22.3 \mathrm{~m}(73 \mathrm{ft})$ of cement was drilled to $966.2 \mathrm{~m}(3,170 \mathrm{ft})$. Fill was cleaned out to $1,052.8 \mathrm{~m}(3,454 \mathrm{ft})$, and the hole was advanced to $1,573.7 \mathrm{~m}(5,163 \mathrm{ft})$ on August 24, 2002, averaging $3 \mathrm{~m}$ (10 ft) of fill on most connections. Tight hole conditions and intermittent fluid returns were encountered below $1457.9 \mathrm{~m}(4,783 \mathrm{ft})$, and at 1,573.7 $\mathrm{m}(5,163 \mathrm{ft})$ the hole became tight and the bit would not rotate except when extra mud was pumped to unload cuttings from the hole. As the crew pulled the drill pipe up into the $13 \mathrm{~d}$-in. casing, the bit began to stick again through the interval 
$1,493.5$ to $1,406.7 \mathrm{~m}$ (4,900 to $4,615 \mathrm{ft})$. When the drill pipe was lowered, a tight spot was encountered at 1,132.9 $\mathrm{m}(3,717 \mathrm{ft})$, requiring reaming of a $37.8-\mathrm{m}$ (124-ft) length of hole past this spot.

Circulation could not be broken after the connection at 1,152.4 $\mathrm{m}(3,781 \mathrm{ft})$. When the crew removed the drill pipe from the hole they found that the bit was plugged with pieces of rubber from the inner liner of a 2-in. hose running from the mud pump to the suction pit. After the bit was unplugged and the source of the rubber determined, the crew cleaned out the mud pits and pumps, mixed more mud, and lowered the 121/4-in. bit back into the hole. The bit could not be advanced through a tight spot at 1,110.1 $\mathrm{m}(3,642 \mathrm{ft})$, so the drill pipe was again removed and the bit inspected. The drillers cleaned clay and fine sand that were blocking a jet on the bit, and lowered the drill pipe back into the hole, tagging fill at 1,116.2 $\mathrm{m}(3,662 \mathrm{ft})$. The drillers reamed and conditioned the hole, then pulled the drill pipe up a short distance to mix more mud. When they lowered the bit it tagged fill at 1,482.2 $\mathrm{m}$ (4,863 ft). The crew then removed the drill string from the hole in preparation for geophysical logging operations and installation of a string of $9 \mathrm{e}$-in. casing to seal off the sloughing section of the hole.

Geophysical logging was conducted on August 27 and 28, 2002, and then a casing subcontractor ran a string of $9 \mathrm{e}$-in. casing, landing it at the depth of $1,477.9 \mathrm{~m}(4,848.8 \mathrm{ft})$. The bottom of the casing was cemented with neat type II cement from inside the $9 \mathrm{e}$-in. casing using the drill pipe with a stab-in adaptor. After this cement job, the drillers attached an $83 / 4$-in. bit and lowered the BHA into the hole to the top of the cement at $(4,809 \mathrm{ft})$. The crew drilled cement from $1,465.8$ to $1,478.3 \mathrm{~m}(4,809$ to $4,850 \mathrm{ft})$ and cleaned out fill to the depth of $1,573.7 \mathrm{~m}$ (5,163 ft). On September 5, 2002, the hole had advanced to $1,900.1 \mathrm{~m}(6,234 \mathrm{ft})$ with no fill on connections, and after a bit change, no fill was encountered when the BHA was lowered back into the hole.

Drilling reached the depth of 2,005.0 $\mathrm{m}$ (6,578 ft) on September 7, 2002, at which point the hole began to pressure up. As the hole was advanced to the depth of 2,033.3 $\mathrm{m}(6,671 \mathrm{ft})$ it began to take as much as 1 to 2 hours to break circulation after making a connection, and the hole was estimated to be producing water at a rate of about 1,892 lpm (500 gpm). Efforts were made to relieve the fluid pressure, and finally the crew removed the drill string to check the bit, but found that it was not plugged and was operating properly. When the crew lowered the drill string back into the hole on September 9, 2002, they encountered fill at 2,020.8 m (6,630 ft), but after this was removed, drilling of the 22.2-cm (8.75-in.) hole continued to the TD of 2,133.6 m (7,000 ft), reached on September 11, 2002. 
The directional survey run inside the $9 e$-in. casing on December 7, 2002, indicates that at the lowest surveyed depth of 2,017.9 $\mathrm{m}(6,617 \mathrm{ft})$ the hole had drifted $57.9 \mathrm{~m}(190 \mathrm{ft})$ to the west of the collar location, and that the hole is relatively straight (no "dog-legs").

A graphical depiction of drilling parameters including penetration rate, revolutions per minute, pump pressure, and weight on the bit is presented in Appendix A-1. See Appendix A-2 for a listing of tubing and casing materials. Drilling fluids and cements used in Well ER-5-4\#2 are listed in Appendix A-3.

\subsubsection{Drilling Problems}

The primary drilling problem on Well ER-5-4\#2 was borehole instability, especially below the water table at the depth of about $221.3 \mathrm{~m}$ (726 ft), despite the use of a bentonite mud mix. Moderate to large amounts of material that had sloughed from the borehole wall were encountered on most connections and at other times when drilling was halted (bit changes, mud-mixing, etc.). In addition, several intervals of the borehole were cemented and then re-drilled in the effort to create stable hole conditions. This resulted in

additional drilling delays, complicated the collection of cuttings samples, and created zones in the borehole for which no representative geophysical log data could be collected. Borehole instability also necessitated the installation of additional casing strings to isolate unstable portions of the borehole and create better drilling conditions.

Large amounts of drill fluid were lost to the formation, especially from the depth of about $265 \mathrm{~m}(870 \mathrm{ft})$, and at other locations. This caused delays in drilling while mud was mixed, and it severely impeded collection of representative drill cuttings samples.

\subsubsection{Fluid Management}

This section provides a summary of fluid management activities during drilling operations at Well ER-5-4\#2. Much of the information presented is from IT (2003) where additional fluid management information is available. Fluids and drill cuttings produced during drilling operations at Well ER-5-4\#2 were managed according to the UGTA FMP (DOE/NV, 1999) and associated state-approved waivers (Liebendorfer, 2000).

To manage the drilling fluids and anticipated water production, 2 sumps (infiltration basins) were constructed prior to drilling of Well ER-5-4, as described in Section 2.2.4 (see also Figure 1-2). No modifications were made to the sump system for drilling of Well ER-5-4\#2. No contaminants were expected during drilling at this site, so neither sump was lined. 
Water used to prepare drilling fluids came from a fill stand at the RWMS in Area 5 and Water Well 5B at the Hazardous Materials Spill Center, also in Area 5. Water from various NTS water wells on the site water well system feeds the RWMS fill stand, which may contain a variable mix of waters from Water Well 4 or Water Well 4a. Lithium bromide was added to the drill fluid as a tracer to provide a means of estimating groundwater production. The rate of water inflow was estimated from the dilution of the tracer in the drill fluid returns. However, loss of circulation and use of bentonite mud prevented monitoring of LiBr dilution in several intervals.

Drilling effluent was monitored according to the methods prescribed in the UGTA FMP (DOE/NV, 1999). Samples of drilling effluent were tested onsite hourly for the presence of tritium, and every 8 hours for lead. Onsite monitoring results indicate that tritium remained at or below background levels, ranging from 0 to $1,792 \mathrm{pCi} / \mathrm{L}$ (IT, 2003). No lead was detected above the detection limit of 50 parts per billion.

The fluid management reporting form for Well ER-5-4 \#2 is provided in Appendix B. The form lists volumes of solids (drill cuttings) and fluids produced during well-construction operations (vadose- and saturated-zone drilling only; well development and aquifer testing will be conducted as a separate initiative). The volume of solids produced was calculated using the diameter of the borehole (from caliper $\operatorname{logs}$ ) and the depth drilled, and includes added volume attributed to a rock bulking factor. The volumes of fluids listed on the report are estimates of total fluid production, and do not account for any infiltration or evaporation of fluids from the sumps.

\subsection{Geologic Data Collection}

This section describes the sources of geologic data obtained from Well ER-5-4\#2 and the methods of data collection. Geologic data collected at Well ER-5-4\#2 consist of drill cuttings, sidewall core samples, and geophysical logs. Data collection, sampling, transfer, and documentation activities were performed according to applicable contractor procedures.

\subsubsection{Collection of Drill Cuttings}

Composite drill cuttings were collected from Well ER-4-5\#2 at 3-m (10-ft) intervals as drilling progressed from the bottom of the conductor hole at $35.1 \mathrm{~m}(115 \mathrm{ft})$ to the TD of the well at 2,133.6 $\mathrm{m}(7,000 \mathrm{ft})$. Triplicate samples, each consisting of approximately $550 \mathrm{cc}$ of material, were collected from 614 intervals. However, in some portions of the hole the volume of cuttings was low, and some samples are composites of materials from intervals greater than $3 \mathrm{~m}(10 \mathrm{ft})$. No samples were collected in 75 intervals where drill fluid was not returned to the surface due to lost circulation. The drill cuttings samples are stored under 
secure, environmentally controlled conditions at the USGS Geologic Data Center and Core Library in Mercury, Nevada. One of these sample sets was sealed with custody tape at the rig site and remains sealed as an archive sample; one set was left unsealed in the original sample containers; and the third set was washed and stored according to standard USGS Core Library procedures. The washed set was used by BN to construct the detailed lithologic log presented in Appendix C. The IT field representative collected an additional 2 sets of reference drill cuttings samples (approximately $15 \mathrm{cc}$ each) from each of the cuttings intervals. One set was examined at the drill site for use in preparing field lithologic descriptions, and remains in the custody of Stoller-Navarro. The other set was sent to Giday WoldeGabriel at LANL for petrographic, mineralogic, and chemical analyses where it remains (see Section 3.3.3).

\subsubsection{Sidewall Core Samples}

The objective of the sampling operation was to acquire geologic samples to supplement very poor quality drill cuttings samples in Well ER-5-4. Sample locations were selected by the IT field representative on the basis of field lithologic logs (with consideration of borehole conditions determined from caliper logs). Table 3-2 lists the recovery and stratigraphic assignment for the two samples recovered. Twenty-four sidewall core samples were attempted by Halliburton Energy Services using a percussion gun tool in the interval $1,306.1$ to $1,475.2 \mathrm{~m}(4,285$ to $4,840 \mathrm{ft})$. The operation was mostly unsuccessful, resulting in only 2 partial cores of rock recovered. Eleven attempts resulted in misfires and ten core barrels were lost in the hole.

Table 3-2

Percussion Gun Sidewall Core samples from Well ER-5-4\#2

\begin{tabular}{|c|c|c||}
\hline \hline $\begin{array}{c}\text { Core Depth } \\
\text { meters (feet) }\end{array}$ & $\begin{array}{c}\text { Length Recovered } \\
\text { centimeters (inches) }\end{array}$ & Stratigraphic Unit \\
\hline \hline $1,366.4(4,483)$ & $2.0(0.8)$ & \multirow{2}{*}{ Wahmonie Formation } \\
\hline $1,414.0(4,639)$ & $3.5(1.4)$ & \\
\hline
\end{tabular}




\subsubsection{Sample Analysis}

Twenty-six samples of drill cuttings from various depths in Well ER-5-4\#2 were submitted to the LANL Earth and Environmental Sciences Division - Geology and Geochemistry laboratories for petrographic, mineralogic, and chemical analyses to aid in stratigraphic identification and for characterization of mineral alteration (WoldeGrabriel et al., 2003). The status of the analyses is listed in Table 3-3. Information from the analyses was incorporated into the detailed lithologic logs presented in Appendix $\mathrm{C}$ and the geologic discussions in Section 4.0.

\subsubsection{Geophysical Data}

Geophysical logs were run in the borehole to further characterize the lithology, structure, and water content of the rocks encountered, and to evaluate borehole conditions. Geophysical logging was conducted in 2 stages during drilling: prior to setting $9 \mathrm{e}$-in. intermediate casing and prior to setting the $5 \frac{1}{2} 2$-in. completion string. No logs were run above approximately $914.4 \mathrm{~m}$ (3,000 ft) in Well ER-5-4\#2 because log data had previously been acquired from this interval in nearby Well ER-5-4. A complete listing of the logs, dates run, depths, and service companies is provided in Table 3-4. The logs are available from BN in Mercury, Nevada, and copies are on file at the Stoller-Navarro office in Las Vegas, Nevada. Preliminary geophysical data from the logs are reproduced in Appendix D.

\subsection{Hydrology of Well ER-5-4\#2}

\subsubsection{Preliminary Water-Level Information}

The elevation of the water table at Well ER-5-4\#2 was projected to be the same as that for Well ER-5-4, approximately $734 \mathrm{~m}(2,407 \mathrm{ft})$. This corresponds to an expected depth to water at approximately $220 \mathrm{~m}$ (720 ft) (IT, 2000). Fluid levels in the well varied considerably during drilling, and the last measurement made during drilling and completion activities found fluid at the depth of $215.7 \mathrm{~m}$ (707.6 ft) (IT, 2003). This measurement was made a week after the completion strings were installed and drilling mud was airlifted from the well, and probably does not represent the actual stabilized water level at this location. Hydrologic studies planned for this well cluster will likely provide a more accurate water level for this site.

\subsubsection{Water Production}

Water production was estimated during drilling of Well ER-5-4\#2 on the basis of LiBr dilution data as measured by IT field personnel. Measurable water production of less than $95 \mathrm{lpm}$ (25 gpm) was first noted at a depth of $221.6 \mathrm{~m}(727 \mathrm{ft})$. Water production ranged from approximately 76 to $1,135 \mathrm{lpm}$ 
Table 3-3

Status of Rock Sample Analyses for Well ER-5-4\#2

\begin{tabular}{|c|c|c|c|c|c|c|}
\hline \multirow{3}{*}{$\begin{array}{c}\text { Depth }{ }^{\mathrm{a}} \\
\text { meters (feet) }\end{array}$} & \multicolumn{6}{|c|}{ Analyses Performed ${ }^{\mathrm{b}}$} \\
\hline & \multicolumn{2}{|c|}{ Petrographic } & \multicolumn{2}{|c|}{ Mineralogic } & \multicolumn{2}{|c|}{ Chemical } \\
\hline & PTS & SEM & MP & XRD & XRF & $\mathrm{Fe}^{2+} / \mathrm{Fe}^{3+}$ \\
\hline $435.9(1,430)$ & C & -- & $\mathrm{C}$ & -- & -- & -- \\
\hline $771.1(2,530)$ & -- & -- & -- & -- & -- & -- \\
\hline $859.5(2,820)$ & -- & -- & -- & -- & -- & -- \\
\hline $1,100.3(3,610)$ & $\mathrm{C}$ & -- & -- & -- & -- & -- \\
\hline $1,140.0(3,740)$ & C & -- & -- & C & C & $\mathrm{C}$ \\
\hline $1,176.5(3,860)$ & $\mathrm{C}$ & -- & $\mathrm{C}$ & $\mathrm{C}$ & $\mathrm{C}$ & $\mathrm{C}$ \\
\hline $1,197.9(3,930)$ & $\mathrm{C}$ & -- & -- & $\mathrm{C}$ & $\mathrm{C}$ & $\mathrm{C}$ \\
\hline $1,222.2(4,010)$ & $\mathrm{C}$ & -- & -- & $\mathrm{C}$ & $\mathrm{C}$ & $\mathrm{C}$ \\
\hline $1,268.0(4,160)$ & $\mathrm{C}$ & -- & -- & $\mathrm{C}$ & C & $\mathrm{C}$ \\
\hline $1,332.0(4,370)$ & $\mathrm{C}$ & -- & -- & $\mathrm{C}$ & $\mathrm{C}$ & $\mathrm{C}$ \\
\hline $1,380.7(4,530)$ & C & -- & -- & $\mathrm{C}$ & C & $\mathrm{C}$ \\
\hline $1,517.9(4,980)$ & $\mathrm{C}$ & -- & -- & -- & -- & -- \\
\hline $1,612.4(5,290)$ & $\mathrm{C}$ & -- & C & C & C & $\mathrm{C}$ \\
\hline $1,673.4(5,490)$ & $\mathrm{C}$ & -- & -- & $\mathrm{C}$ & $\mathrm{C}$ & C \\
\hline $1,694.7(5,560)$ & $\mathrm{C}$ & -- & -- & $\mathrm{C}$ & $\mathrm{C}$ & $\mathrm{C}$ \\
\hline $1,740.4(5,710)$ & $\mathrm{C}$ & -- & -- & $\mathrm{C}$ & $\mathrm{C}$ & $\mathrm{C}$ \\
\hline $1,764.8(5,790)$ & $\mathrm{C}$ & -- & -- & $\mathrm{C}$ & $\mathrm{C}$ & $\mathrm{C}$ \\
\hline $1,813.6(5,950)$ & $\mathrm{C}$ & -- & -- & C & $\mathrm{C}$ & $\mathrm{C}$ \\
\hline $1,841.0(6,040)$ & $\mathrm{C}$ & -- & -- & $\mathrm{C}$ & $\mathrm{C}$ & $\mathrm{C}$ \\
\hline $1,902.0(6,240)$ & $\mathrm{C}$ & -- & $\mathrm{C}$ & C & $\mathrm{C}$ & $\mathrm{C}$ \\
\hline $1,935.5(6,350)$ & $\mathrm{C}$ & -- & -- & $\mathrm{C}$ & $\mathrm{C}$ & C \\
\hline $1,953.8(6,410)$ & $\mathrm{C}$ & -- & $\mathrm{C}$ & C & $\mathrm{C}$ & $\mathrm{C}$ \\
\hline $1,999.5(6,560)$ & $\mathrm{C}$ & -- & -- & C & C & $\mathrm{C}$ \\
\hline $2,069.6(6,790)$ & C & -- & -- & C & C & C \\
\hline $2,100.1(6,890)$ & $\mathrm{C}$ & -- & -- & $\mathrm{C}$ & C & C \\
\hline $2,133.6(7,000)$ & $\mathrm{C}$ & -- & -- & $\mathrm{C}$ & $\mathrm{C}$ & $\mathrm{C}$ \\
\hline
\end{tabular}

a All samples are from drill cuttings. Depth represents base of 3-m (10-ft) sample interval for drill cuttings.

b Analyses performed by the research laboratory of the Hydrology, Geochemistry, and Geology Group of the Earth and Environmental Sciences Division at LANL. $\mathbf{C}=$ analysis complete; -- = analysis not planned. Analysis type: PTS = polished thin section; SEM = scanning electron microscopy; $\mathbf{M P}=$ electron microprobe; $\mathbf{X R D}=\mathrm{x}$-ray diffraction; $\mathbf{X R F}=\mathrm{x}$-ray fluorescence; $\mathbf{F e}^{2+} / \mathrm{Fe}^{3+}=$ wet chemical analysis for iron. 
Table 3-4

Well ER-5-4\#2 Geophysical Log Summary

\begin{tabular}{|c|c|c|c|c|c|c|}
\hline Geophysical Log Type a & Log Purpose & $\begin{array}{l}\text { Logging } \\
\text { Service }\end{array}$ & $\begin{array}{l}\text { Date } \\
\text { Logged }\end{array}$ & Run Number & $\begin{array}{l}\text { Bottom of } \\
\text { Logged } \\
\text { Interval }^{c} \\
\text { meters (feet) }\end{array}$ & $\begin{array}{l}\text { Top of Logged } \\
\text { Interval }^{\mathbf{c}} \\
\text { meters (feet) }\end{array}$ \\
\hline *Natural Gamma Ray Spectroscopy & $\begin{array}{l}\text { Stratigraphic correlation, lithologic } \\
\text { determination, mineralogy, natural } \\
\text { and man-made radiation, fracture } \\
\text { identification }\end{array}$ & HES & $\begin{array}{l}09 / 13 / 2002 \\
09 / 14 / 2002 \\
09 / 15 / 2002 \\
09 / 15 / 2002\end{array}$ & $\begin{array}{l}\text { SGR-1/GR-8 } \\
\text { SGR-2/GR-9 } \\
\text { SGR-3/GR-10 } \\
\text { SGR-4/GR-11 }\end{array}$ & $\begin{array}{l}1,852.6(6,078) \\
2,035.1(6,677) \\
2,132.1(6,995) \\
2,125.4(6,973)\end{array}$ & $\begin{array}{l}1,452.7(4,766) \\
1,219.2(4,000) \\
1,447.8(4,750) \\
1,447.8(4,750)\end{array}$ \\
\hline *Six Arm Caliper/Gamma Ray & $\begin{array}{l}\text { Borehole conditions, cement } \\
\text { volume calculation, fracture } \\
\text { identification }\end{array}$ & HES & $\begin{array}{l}08 / 28 / 2002 \\
09 / 13 / 2002\end{array}$ & $\begin{array}{l}\text { CA6-1/GR-1 } \\
\text { CA6-2/GR-7 }\end{array}$ & $\begin{array}{l}1,477.8(4,848) \\
2,128.4(6,983)\end{array}$ & $\begin{array}{c}923.5(3,030) \\
1,356.2(4,450)\end{array}$ \\
\hline *Epithermal Neutron/ Density & $\begin{array}{l}\text { Total water content, rock porosity, } \\
\text { stratigraphic correlation, lithologic } \\
\text { determination }\end{array}$ & HES & $\begin{array}{l}08 / 28 / 2002 \\
09 / 14 / 2002 \\
09 / 15 / 2002\end{array}$ & $\begin{array}{l}\text { DSEN-1/ SDL-1/GR-2 } \\
\text { DSEN-2/ SDL-2/GR-10 } \\
\text { DSEN-3/SDL-3 /GR-11 }\end{array}$ & $\begin{array}{l}1,479.5(4,854) \\
2,035.8(6,679) \\
2,131.8(6,994)\end{array}$ & $\begin{array}{c}838.2(2,750) \\
1,219.2(4,000) \\
1,447.8(4,750)\end{array}$ \\
\hline $\begin{array}{l}\text { *Dual Laterolog/ Spontaneous } \\
\text { Potential }\end{array}$ & $\begin{array}{l}\text { Lithologic determination, water } \\
\text { saturation, stratigraphic correlation }\end{array}$ & HES & $\begin{array}{l}08 / 28 / 2002 \\
09 / 14 / 2002\end{array}$ & $\begin{array}{c}\text { DLL- } 1 / \text { SP-1/GR-3 } \\
\text { DLL-2/ SP-2 }\end{array}$ & $\begin{array}{l}1,477.1(4,846) \\
2,126.9(6,978)\end{array}$ & $\begin{array}{c}944.9(3,100) \\
1,447.8(4,750)\end{array}$ \\
\hline Full Wave Sonic - Waveform & Fracture identification & HES & $\begin{array}{l}08 / 28 / 2002 \\
09 / 14 / 2002\end{array}$ & $\begin{array}{l}\text { FWS-1/GR-4 } \\
\text { FWS-2 }\end{array}$ & $\begin{array}{l}1,465.5(4,808) \\
2,117.8(6,948)\end{array}$ & $\begin{array}{c}941.8(3,090) \\
1,447.8(4,750)\end{array}$ \\
\hline${ }^{*}$ Full Wave Sonic - Delta-T & Porosity, seismic properties & HES & $\begin{array}{l}08 / 28 / 2002 \\
09 / 14 / 2002\end{array}$ & $\begin{array}{l}\text { FWS-1/GR-4 } \\
\text { FWS-2 }\end{array}$ & $\begin{array}{l}1,465.5(4,808) \\
2,117.8(6,948)\end{array}$ & $\begin{array}{c}941.8(3,090) \\
1,447.8(4,750)\end{array}$ \\
\hline Temperature & $\begin{array}{l}\text { Groundwater temperature, open } \\
\text { fracture identification }\end{array}$ & HES & $\begin{array}{l}09 / 11 / 2002 \\
09 / 13 / 2002\end{array}$ & $\begin{array}{l}\text { TL-1/GR-5 } \\
\text { TL-2/GR-6 }\end{array}$ & $\begin{array}{l}2,084.8(6,840) \\
2,114.1(6,936)\end{array}$ & $\begin{array}{l}1,341.1(4,400) \\
1,402.1(4,600)\end{array}$ \\
\hline $\begin{array}{l}\text { Percussion Gun Sidewall Coring } \\
\text { Tool }\end{array}$ & Geologic Samples & HES & 08/28/2002 & SWC-1 & $1,475.2(4,840)$ & $1,306.1(4,285)$ \\
\hline Electro-Micro Imager & $\begin{array}{l}\text { Lithologic characterization, } \\
\text { structural analysis, fracture and } \\
\text { void analysis }\end{array}$ & HES & 09/14/2002 & EMI-3/GR-9 & $2,029.1(6,657)$ & $1,486.8(4,878)$ \\
\hline Thermal Flow Meter & $\begin{array}{l}\text { Rate and direction of groundwater } \\
\text { flow in borehole }\end{array}$ & DRI & $12 / 05 / 2002$ & TFM-1 & $2,011.7(6,600)$ & $304.8(1,000)$ \\
\hline Chemistry/ Temperature Log & $\begin{array}{l}\text { Groundwater chemistry and } \\
\text { temperature, formation } \\
\text { transmissivity }\end{array}$ & DRI & $\begin{array}{l}12 / 04 / 2002 \\
12 / 04 / 2002\end{array}$ & $\begin{array}{l}\text { Chem- } 1 / \text { TL-4 } \\
\text { Chem-2/TL-5 }\end{array}$ & $\begin{array}{l}1,819.7(5,970) \\
2,023.9(6,640)\end{array}$ & $\begin{array}{l}224.9(738) \\
224.9(738)\end{array}$ \\
\hline North Seeker Earth-Rate Gyro & $\begin{array}{l}\text { Bottom hole location, true vertical } \\
\text { depth }\end{array}$ & $\mathrm{BHI}$ & $12 / 06 / 2002$ & DRG-1 & $2,016.6(6,617)$ & Surface \\
\hline
\end{tabular}

a Logs presented in geophysical log summary, Appendix D, are indicated by *.

b $\quad$ HES = Halliburton Energy Services; DRI = Desert Research Institute; $\mathbf{B H I}=$ Baker Hughes Inteq

c Depth below ground surface. 
(20 to 300 gpm), but was generally less than 379 lpm (100 gpm) from $221.6 \mathrm{~m}$ (727 ft) to a depth of approximately 1,104.9 $\mathrm{m}(3,625 \mathrm{ft})$. At approximately 1,104.9 $\mathrm{m}(3,625 \mathrm{ft})$, water production increased abruptly to about $568 \mathrm{lpm}(150 \mathrm{gpm})$, and then further increased to1,893 lpm (500 gpm) at approximately 1,325.9 m (4,350 ft), within the welded Rainier Mesa Tuff. Below 1,325.9 m (4,350 ft), production remained generally between 1,136 and 1,514 lpm (300 and 400 gpm) to a depth of about 1,562.1 m $(5,125 \mathrm{ft})$. When drilling resumed at $1,573.7 \mathrm{~m}(5,163 \mathrm{ft})$ after setting of the intermediate casing at $1,477.9 \mathrm{~m}(4,848.8 \mathrm{ft})$, water production dropped precipitously to $38 \mathrm{lpm}$ (10 gpm) and remained at this level to a depth of approximately $1,966.0 \mathrm{~m}(6,450 \mathrm{ft})$. At 1,966.0 $\mathrm{m}(6,450 \mathrm{ft})$ production began to increase rapidly to a maximum rate of 2,158 lpm (570 gpm) at approximately 1,996.4 m (6,550 ft). Geophysical logs and lost circulation during drilling at this depth indicate that the increased water production around 1,996.4 $\mathrm{m}(6,550 \mathrm{ft})$ is likely due to the presence of open fractures within zeolitic nonwelded tuff of the Bullfrog Tuff in this depth interval. Below 2,034.5 m (6,675 ft) production decreased to approximately $1,136 \mathrm{lpm}$ (300 gpm) and then rose steadily to approximately 1,325 lpm (350 gpm) near the TD of the well at 2,133.6 $\mathrm{m}(7,000 \mathrm{ft})$.

Estimated water production rates are presented graphically in Appendix A-1.

\subsubsection{Preliminary Flow Meter Data}

Flow meter data, along with temperature, $\mathrm{EC}$, and $\mathrm{pH}$ measurements, can be used to characterize borehole fluid variability, which may indicate inflow and outflow zones. Typically, these measurements are made before the completion string is installed and the data are consulted during planning of zones to be completed. At Well ER-5-4\#2, these data were collected approximately $2 \frac{1}{2} 2$ months after the completion string was installed, following partial development of the well by pumping (these pumping operations will be described in a separate report). DRI personnel made measurements inside the Well ER-5-4\#2 completion string with their TFM and chemistry tools on December 4 and 5, 2002.

DRI personnel obtained TFM measurements at 10 locations between the depths of 304.8 and 2,011.7 m $(1,000$ and 6,600 ft). Preliminary analysis of these data indicates an upward flow of water within the borehole at the 8 highest stations located between 304.8 and $1,981.2 \mathrm{~m}(1,000$ to $6,500 \mathrm{ft})$. The 2 deepest stations, at 1,996.4 and 2,011.7 $\mathrm{m}$ (6,550 and 6,600 ft), both recorded downward flow. These 2 stations are located opposite large fractures that produced water during drilling.

DRI also ran a chemistry log, which included measurements of temperature, $\mathrm{EC}$, and $\mathrm{pH}$. Two runs of the tool had to be made due to the failure on the first run of the $\mathrm{pH}$ sensor at 1,738.0 $\mathrm{m}(5,702 \mathrm{ft})$ and the 
subsequent failure of all sensors due to the implosion of the $\mathrm{pH}$ sensor at $1,819.7 \mathrm{~m}(5,970 \mathrm{ft})$. After the tool was repaired, the second run was made from 224.9 to $2,023.9 \mathrm{~m}$ (738 to $6,640 \mathrm{ft}$ ) recording only temperature and EC. Groundwater temperature gradually increased from the minimum reading of 30.3 degrees $\mathrm{C}$ ( 86.5 degrees $\mathrm{F}$ ) at the top of the fluid column to the deepest logged depth near the bottom of the completion string. The maximum temperature of 48.7 degrees $\mathrm{C}$ (119.7 degrees F) was measured at 2,025.1 $\mathrm{m}(6,644 \mathrm{ft})$. A slight perturbation in the temperature curve was noted near $1,074.4 \mathrm{~m}(3,525 \mathrm{ft})$, within the $9 \mathrm{e}$-in. intermediate casing, approximately $22.9 \mathrm{~m}(75 \mathrm{ft})$ below the bottom of the $44.5-\mathrm{cm}\left(17 \frac{1}{2}\right.$-in.) diameter portion of the borehole. Changes in the $\mathrm{pH}$ and EC are most notable from $1,432.6$ to $1,524.0 \mathrm{~m}(4,700$ to $5,000 \mathrm{ft})$ near the top of the $5 \frac{1}{2}$-in. production casing.

\subsubsection{Preliminary Groundwater Characterization Samples}

No preliminary groundwater characterization samples were collected from Well ER-5-4\#2 after drilling because of the residual bentonite mud in the borehole, and because it was expected that samples would soon be collected during upcoming development and testing operations.

\subsection{Precompletion and Open-Hole Development}

No precompletion development was conducted in Well ER-5-4\#2 due to borehole instability problems that persisted through geophysical logging operations and installation of the completion string.

\subsection{Well Completion}

Installation of the completion string at Well ER-5-4\#2 took place on September 17 and 18, 2002.

Figure 3-2 is a schematic of the final well-completion design for Well ER-5-4\#2, Figure 3-3 shows a plan view and profile of the wellhead surface completion, and Table 3-4 summarizes construction specifications of the completion string. Data for this section were obtained from daily activity reports and casing records provided by the BN Drilling Department. Information from IT's well data report (IT, 2003) was also consulted for preparation of this section.

\subsubsection{Well Completion Design}

The final completion design differs from the proposed design, as described in the following paragraphs. The departure of the final completion design from that proposed is mainly due to significant differences in the geology encountered at Well ER-5-4\#2 from that predicted prior to drilling (see Section 4.3). 


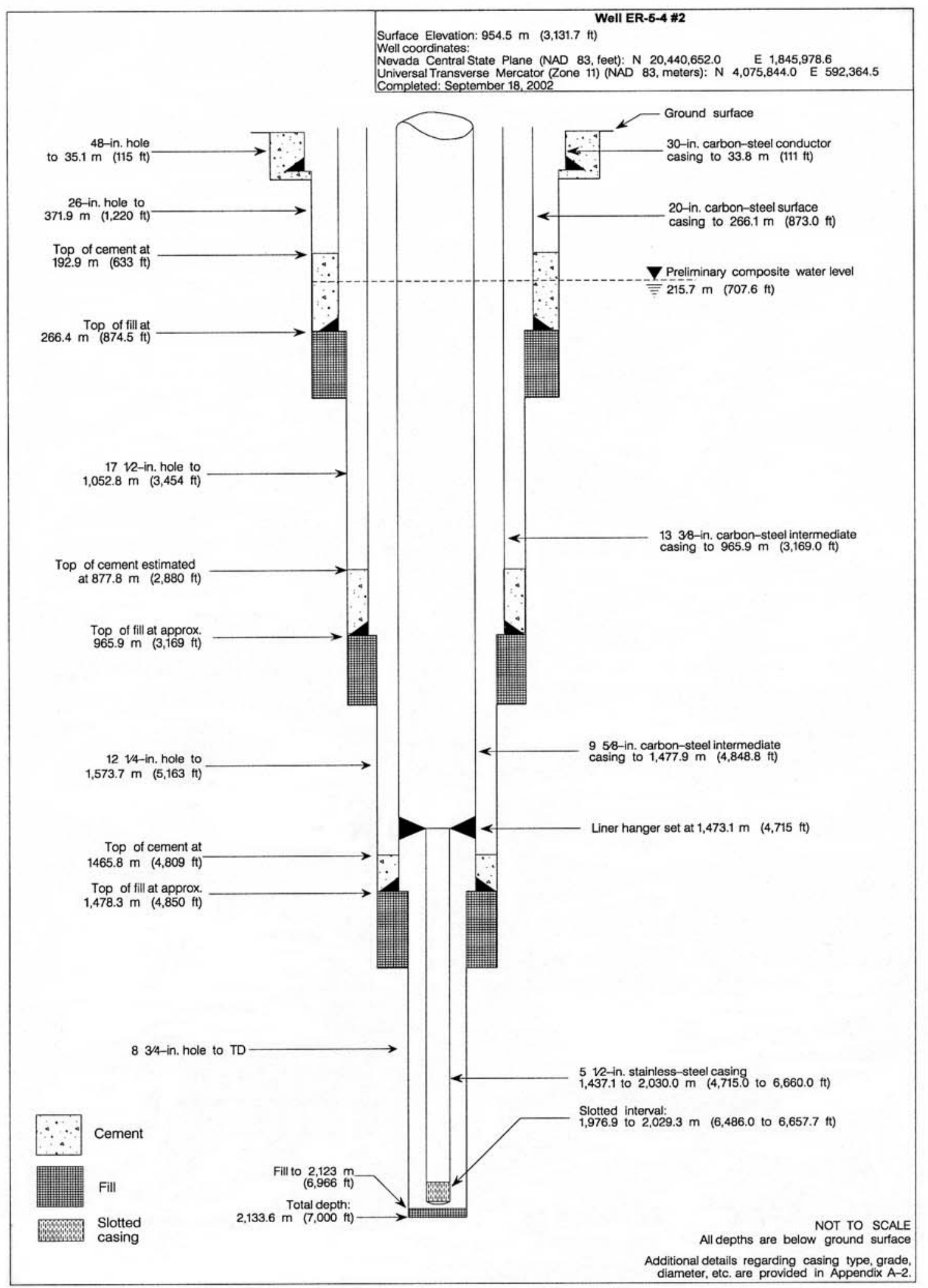

Figure 3-2

As-Built Completion Schematic for Well ER-5-4\#2 


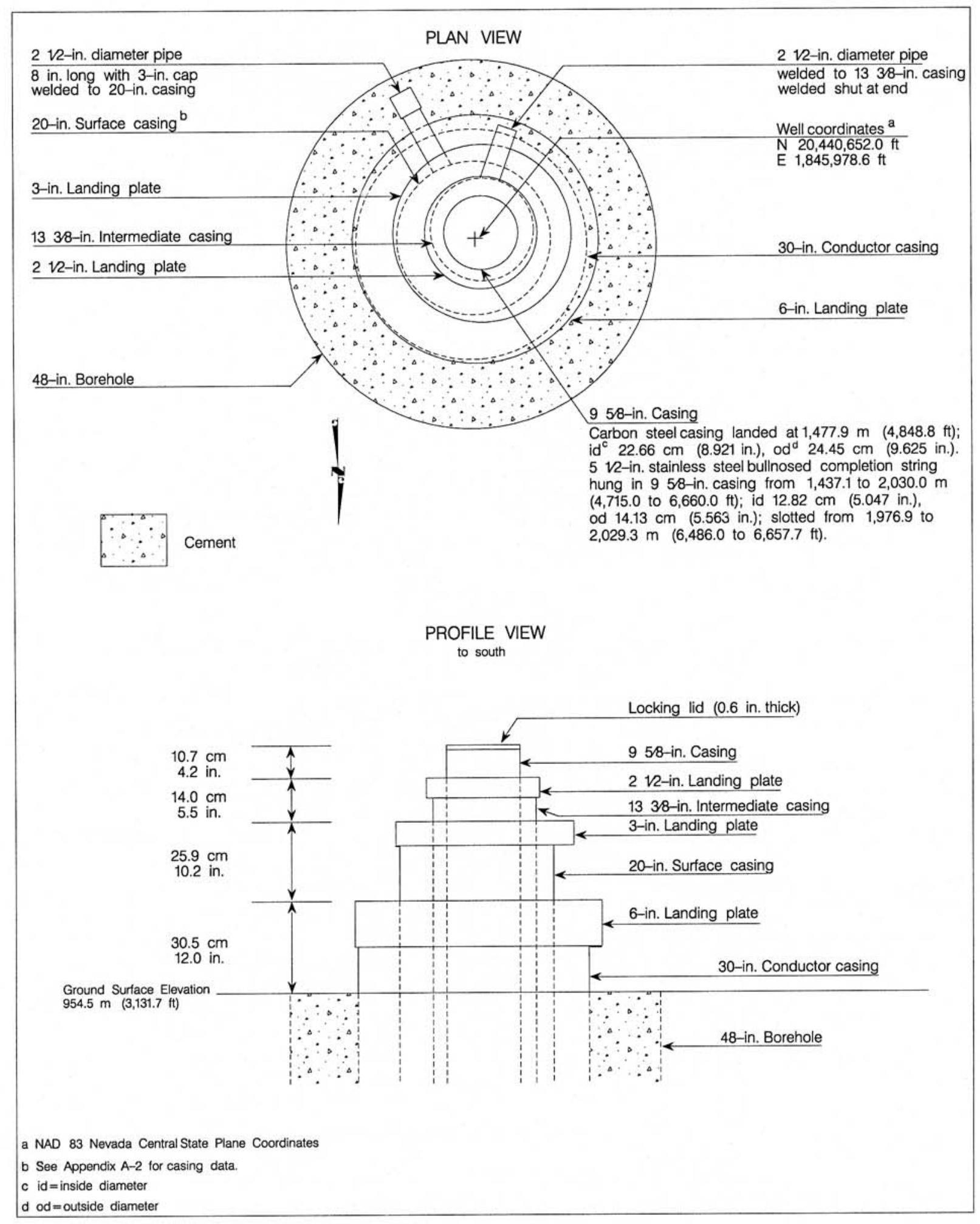

Figure 3-3

Wellhead Diagram for Well ER-5-4\#2 
Table 3-5

\section{Completion String Construction Summary for Well ER-5-4\#2}

\begin{tabular}{|c|c|c|c|c|}
\hline Casing Type & \multicolumn{2}{|c|}{$\begin{array}{c}\text { Configuration } \\
\text { meters (feet) } \\
\end{array}$} & Cement & Sand/Gravel \\
\hline \multirow{3}{*}{$\begin{array}{c}51 / 2 \text {-in. } \\
\text { Stainless-steel } \\
\text { production } \\
\text { casing }^{a}\end{array}$} & \multirow{3}{*}{$\begin{array}{l}1,437.1 \text { to } 2,030 \\
(4,715 \text { to } 6,660)\end{array}$} & $\begin{array}{c}\text { Blank } \\
1,437.1 \text { to } 1,976.9 \\
(4,715.0 \text { to } 6,486.0) \\
\end{array}$ & \multirow{3}{*}{ None } & \multirow{3}{*}{ None } \\
\hline & & $\begin{array}{c}4 \text { consecutive slotted joints } \\
1,976.9 \text { to } 2,029.3 \\
(6,486.0 \text { to } 6,657.7)\end{array}$ & & \\
\hline & & $\begin{array}{c}\text { Bull nose } \\
2,029.3 \text { to } 2,030) \\
(6,657.7 \text { to } 6,660.0)\end{array}$ & & \\
\hline
\end{tabular}

a Top of 51/2-in. casing is suspended from a liner hanger set at 1,437.1 $\mathrm{m}(4,715 \mathrm{ft})$ within the 9 e -in. carbonsteel intermediate casing.

\subsubsection{Proposed Completion Design}

The proposed completion design (IT, 2001a) was based on the assumption that the well would penetrate completely through the volcanic rocks, which form a thick tuff confining unit, and reach TD at 1,889.8 m $(6,200 \mathrm{ft})$ within the underlying Paleozoic-age carbonate rocks, which compose the lower carbonate aquifer. The well was planned to be constructed with 2 completion zones, one in the middle portion of the tuff confining unit, and the other at the top of the lower carbonate aquifer. A string of 51/2-in. stainless-steel casing (suspended from carbon-steel 7e -in. casing) with a slotted interval at 1,828.8 to 1,886.7 $\mathrm{m}(6,000$ to $6,190 \mathrm{ft}$ ) was planned to provide hydrologic access to the lower carbonate aquifer. A string of $2 \mathrm{f}$-in. tubing with a $30-\mathrm{m}$ (100-ft) slotted interval was planned to be installed (outside the production casing) in the middle of the tuff confining unit.

\subsubsection{As-Built Completion Design}

The design of the Well ER-5-4\#2 completion was determined through consultation with members of the UGTA TWG, on the basis of onsite evaluation of data such as lithology and water production, drilling data (lost circulation, etc.), and data from various geophysical logs.

Drilling at Well ER-5-4\#2 was terminated at 2,133.6 m (7,000 ft) (243.8 m [800 ft] below the planned TD) while still in the tuff confining unit. A single completion zone was placed within the tuff confining unit (Figure 3-2, see also Figure 4-3). The composition of the completion string summarized here is also 
provided in Table 3-5, and the casing materials are listed in Appendix A-2. The string consists of $169.7 \mathrm{~m}$ $(556.9 \mathrm{ft})$ of stainless-steel 51/2-in. casing. The top of the string hangs from a liner hanger set at $1,437.1 \mathrm{~m}$ $(4,715.0 \mathrm{ft})$ within the $9 \mathrm{e}$-in. carbon-steel intermediate casing. The bottom of the casing terminates in a bull nose at 2,030.0 $\mathrm{m}(6,660.0 \mathrm{ft})$.

The slotted interval consists of 4 consecutive slotted joints placed at 1,976.9 to 2,029.3 $\mathrm{m}(6,486$ to $6,657.7 \mathrm{ft})$. The openings in each slotted casing joint are 0.198 to $0.203 \mathrm{~cm}(0.078$ to $0.080 \mathrm{in}$.) wide and $5.08 \mathrm{~cm}$ ( 2 in.) long, cut in rings of 18 slots (spaced 20 degrees apart around the joint). The rings are spaced $15.2 \mathrm{~cm}$ (6 in.) apart, and the longitudinal centers of the slots in each ring are staggered 10 degrees from the slot centers in the next ring. No gravel-pack or cement was utilized in the completion to isolate the slotted interval because only a single hydrogeologic unit, the tuff confining unit, was encountered below the base of the $9 \mathrm{e}$-in. intermediate casing. However, the slotted interval does coincide with a fractured interval within the tuff confining unit that appeared to produce water during drilling (see Sections 4.2.1 and 4.4).

\subsubsection{Rationale for Differences between Actual and Proposed Well Design}

The proposed well design was based on the expectation that the Well ER-5-4\#2 borehole would penetrate completely through the tuff confining unit and terminate within the lower carbonate aquifer. Because the well did not penetrate through the tuff confining unit as expected, a completion zone within the lower carbonate aquifer was not possible. A single completion zone was placed within the tuff confining unit as generally planned.

\subsubsection{Well Completion Method}

Well construction materials were inspected according to relevant procedures; standard decontamination procedures were employed to prevent the introduction of contaminants into the well. The 51/2-in. casing and liner hanger were lowered into the hole on 5-in. drill pipe. After the liner hanger was set at 1,473.1 m $(4,715 \mathrm{ft})$, the 5 -in. drill pipe was detached from the liner hanger. The hole was then unloaded by pumping clear water down the 5-in. drill pipe. When clear water was observed at the discharge line, unloading was terminated and the 5-in. drill pipe was removed from the hole. No gravel pack or cement was used in the completion.

The drill rig was released after the completion string was installed. Hydrologic testing was planned as a separate effort, so a pump was not installed in the well and no well development or pumping tests were conducted immediately after completion. 


\subsection{Actual versus Planned Costs and Scheduling}

The original BN cost model developed for Well ER-5-4\#2 was based on drilling to the planned TD of $1,889.8 \mathrm{~m}(6,200 \mathrm{ft})$. The drilling program baseline projected that it would require 53 days to drill and complete the well. However, as with Well ER-5-4, the actual conditions encountered during drilling of the well (severe borehole instability; deeper than planned TD) were measurably different from predicted conditions so the baseline was changed during drilling.

The new cost model is based on the actual TD of 2,133.6 m (7,000 ft). It took 69 days to accomplish drilling of the surface and main holes, logging, and completion of the well, after construction of the conductor hole by BN. Drilling of the surface hole and installation of the 20-in. surface casing proceeded as expected. However, drilling of the production hole and installation of the completion casing took longer than predicted, and required additional cementing and re-drilling of segments of the borehole, and installation of an intermediate casing string. A graphical comparison, by day, of planned and actual wellconstruction activities is presented in Figure 3-4.

The cost analysis for Well ER-5-4\#2 begins with the repositioning of the UDI drill rig on the Well Cluster ER-5-4 pad after drilling of Well ER-5-4. The cost of building roads, the drill pad, and sumps is not included, and the cost of well-site support by IT is not included. The total construction cost for Well ER-5-4\#2 includes all drilling costs: charges by the drilling subcontractor; charges by other support subcontractors (including compressor services, drilling fluids, bits, casing services, down-hole tools, and geophysical logging); and charges by $\mathrm{BN}$ for mobilization and demobilization of equipment, construction of the conductor hole, cementing services, completion materials, radiation technicians, inspection services, and geotechnical consultation.

The total planned cost for constructing Well ER-5-4\#2, based on the new baseline developed because the actual conditions differed greatly from the expected conditions, was $\$ 5,607,398$. The actual cost was $\$ 5,357,674$, or 4.5 percent less than the planned cost. Figure 3-5 presents a comparison of the planned (new baseline) and actual costs, by day, for drilling and completing Well ER-5-4\#2. 


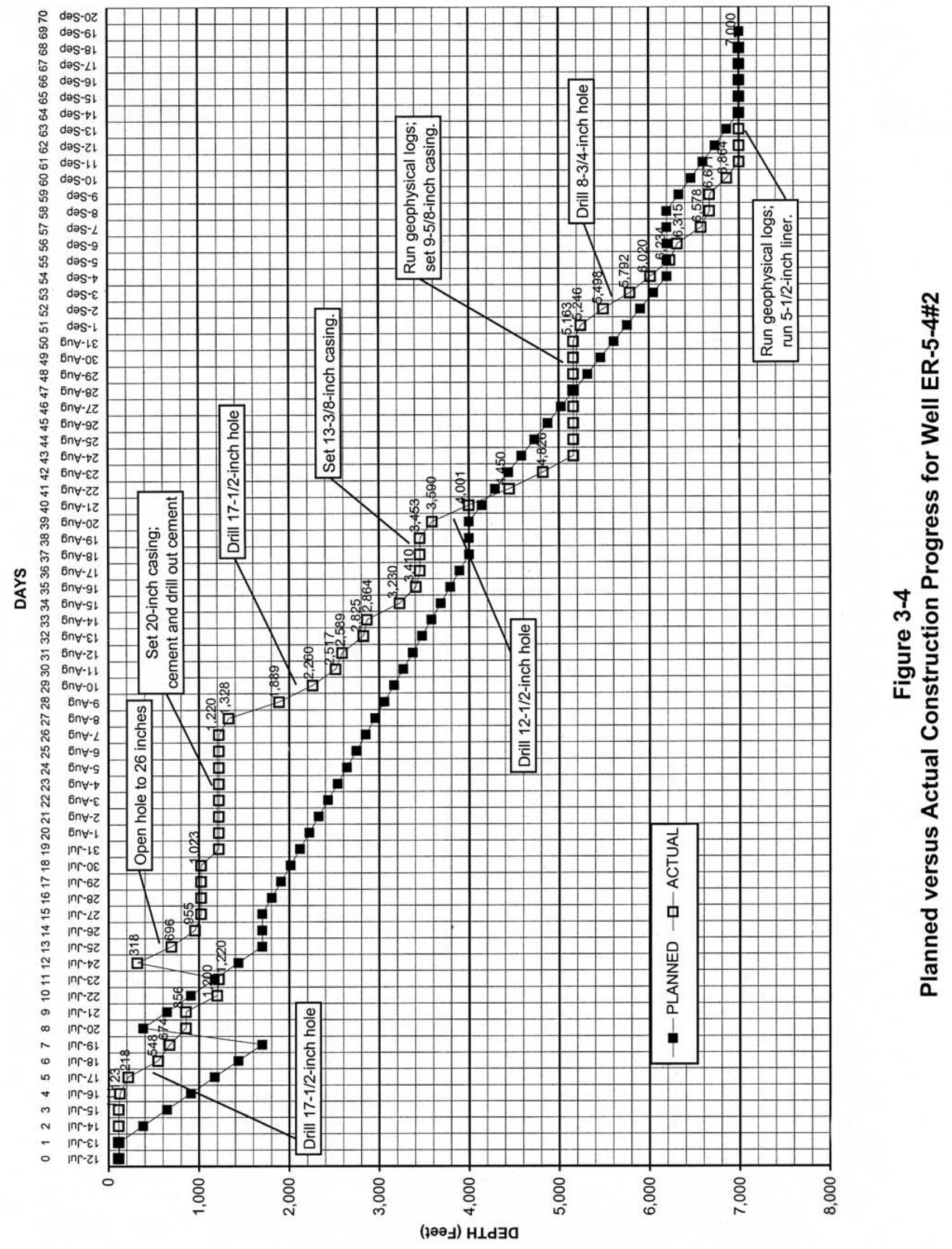




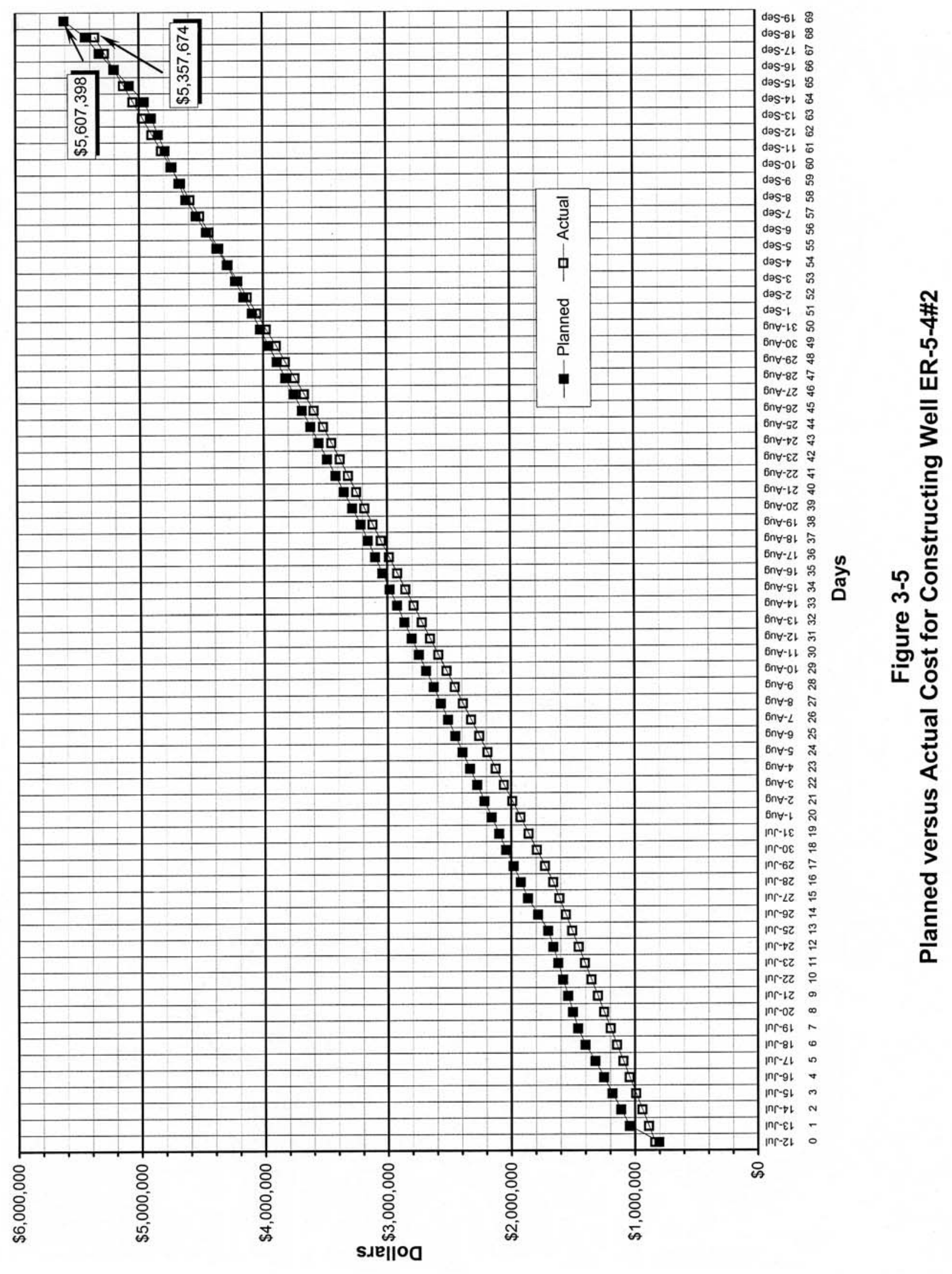




\subsection{Summary, Recommendations, and Lessons Learned}

\subsubsection{Summary for Well ER-5-4\#2}

Main hole drilling at Well ER-5-4 \#2 commenced on July 16, 2002, and concluded on September 11, 2002 , at a total drilled depth of 2,133.6 $\mathrm{m}(7,000 \mathrm{ft})$. Sloughing of the borehole wall, particularly in the upper portions of the hole, resulted in difficult drilling conditions and necessitated numerous borehole cleaning and conditioning efforts, placing of cement plugs and re-drilling portions of the hole, setting of 2 additional strings of casing, and required the use of bentonite-based mud as a circulating fluid. A single completion string consisting of $51 / 2$-in. stainless steel casing, slotted from 1,976.9 to 2,020.3 m (6,486 to 6,657.7 ft), was installed in the hole.

Geologic data collected during drilling include composite drill cuttings samples collected every $3 \mathrm{~m}(10 \mathrm{ft})$ from $35.1 \mathrm{~m}(115 \mathrm{ft})$ to TD, and 2 partial sidewall core samples. Geophysical logging was conducted in 2 stages during drilling: prior to setting $9 \mathrm{e}$-in. intermediate casing and prior to setting the $5 \frac{1}{2} 2$-in. completion string. No logs were run above approximately $914.4 \mathrm{~m}$ (3,000 ft) in Well ER-5-4\#2 because log data had previously been acquired from the equivalent stratigraphic interval in nearby Well ER-5-4.

Well ER-5-4 \#2 is collared in young alluvial deposits, and penetrated 1,120.4 m (3,676 ft) of Quaternary and Tertiary alluvium before encountering $242.6 \mathrm{~m}$ (796 ft) of Tertiary volcanic rocks that consist mainly of unaltered, nonwelded to moderately welded ash-flow tuff. Below the interval of ash-flow tuff the well penetrated an additional $770.5 \mathrm{~m}(2,528 \mathrm{ft})$ of Tertiary volcanic rocks consisting mainly of zeolitic nonwelded tuff. Paleozoic rocks were not encountered and drilling was terminated within volcanic rocks at a depth of 2,133.6 $\mathrm{m}(7,000 \mathrm{ft})$. Tritium levels in the drilling fluid ranged from 0 to 1,792 picocuries per liter during drilling, which is at or below background levels. No other radionuclides above background were encountered in the drilling fluids from Well ER-5-4\#2, and lead was not detected above the detection limit of 50 parts per billion. A preliminary fluid-level was measured at $215.7 \mathrm{~m}(707.6 \mathrm{ft})$ on September 18, 2002, after the completion string was installed.

\subsubsection{Recommendations}

Results from well development and testing activities should be evaluated to better understand the potential for groundwater flow through fractures within the tuff confining unit. All the geologic and hydrologic data from Well ER-5-4\#2 should be integrated with other Frenchman Flat data to refine the Frenchman Flat hydrostratigraphic framework model (IT, 1998). 


\subsubsection{Lessons Learned from Well ER-5-4\#2}

Borehole instability can cause severe problems when drilling alluvium in the central portion of Frenchman Flat, even when bentonite-based mud is used as a drilling fluid.

Increased down-hole pressures related to the use of bentonite mud as a drilling fluid can cause nearsurface fracturing of poorly consolidated units such as alluvium. These drilling-induced borehole fractures can propagate to the surface and create pathways for drilling fluids to flow out of the borehole and onto the ground surface. 


\subsection{Geology and Hydrogeology}

\subsection{Introduction}

This section summarizes the geology and hydrogeology of Well Cluster ER-5-4. The detailed lithologic logs for both wells are presented in Appendix C. These lithologic descriptions were developed using drill cuttings and sidewall core samples, geophysical logs, and drilling parameters. The Hydrology, Geochemistry, and Geology Group of the Earth and Environmental Sciences Division at LANL performed petrographic, mineralogic, and chemical analyses of select lithologic samples from both wells of the cluster (WoldeGabriel et al., 2003). Results from these analyses were incorporated into the lithologic logs. Regional (i.e., basin-wide) geologic interpretations and analyses provided in this section integrate the well information with surface geology and geophysical data, including gravity (Phelps and Graham, 2002), aeromagnetic (Grauch and Hudson, 1995), and 3-D seismic reflection (Prothro, 2002).

\subsection{Geology}

This section is subdivided into 3 discussions relating to the geology of Well Cluster ER-5-4. Section 4.2.1 describes the geologic setting of Frenchman Flat and the well cluster. The stratigraphic and lithologic units penetrated at the well cluster are discussed in Section 4.2.2. Because of the significant influence some alteration products have on the hydraulic properties of certain rocks, alteration of the rocks encountered at the well cluster is discussed separately in Section 4.2.3. More detailed descriptions of the stratigraphy, lithology, and alteration of the rocks encountered are provided in Appendix C.

\subsubsection{Geologic Setting}

Well Cluster ER-5-4 lies on a very gently southeast-sloping surface composed of young alluvium (Figure 4-1) in the central portion of Frenchman Flat. Frenchman Flat is a hydrologically closed, Cenozoic-age basin formed in response to basin-and-range extension. Topographically, the basin is ovalshaped, elongated in a northeast direction, and contains the Frenchman Lake playa that marks the topographic low point of the basin. The well cluster is located approximately $1.6 \mathrm{~km}(1 \mathrm{mi})$ northwest of the Frenchman Lake playa (“Qp" on Figure 4-1).

Rocks exposed in the highlands around the margins of Frenchman Flat consist of Tertiary-age volcanic and tuffaceous sedimentary rocks that overlie complexly folded and faulted Paleozoic-age sedimentary rocks (Hinrichs and McKay, 1965; Poole, 1965; Poole et al., 1965; Hinrichs, 1968; McKeown et al., 1976; 
This page intentionally left blank. 


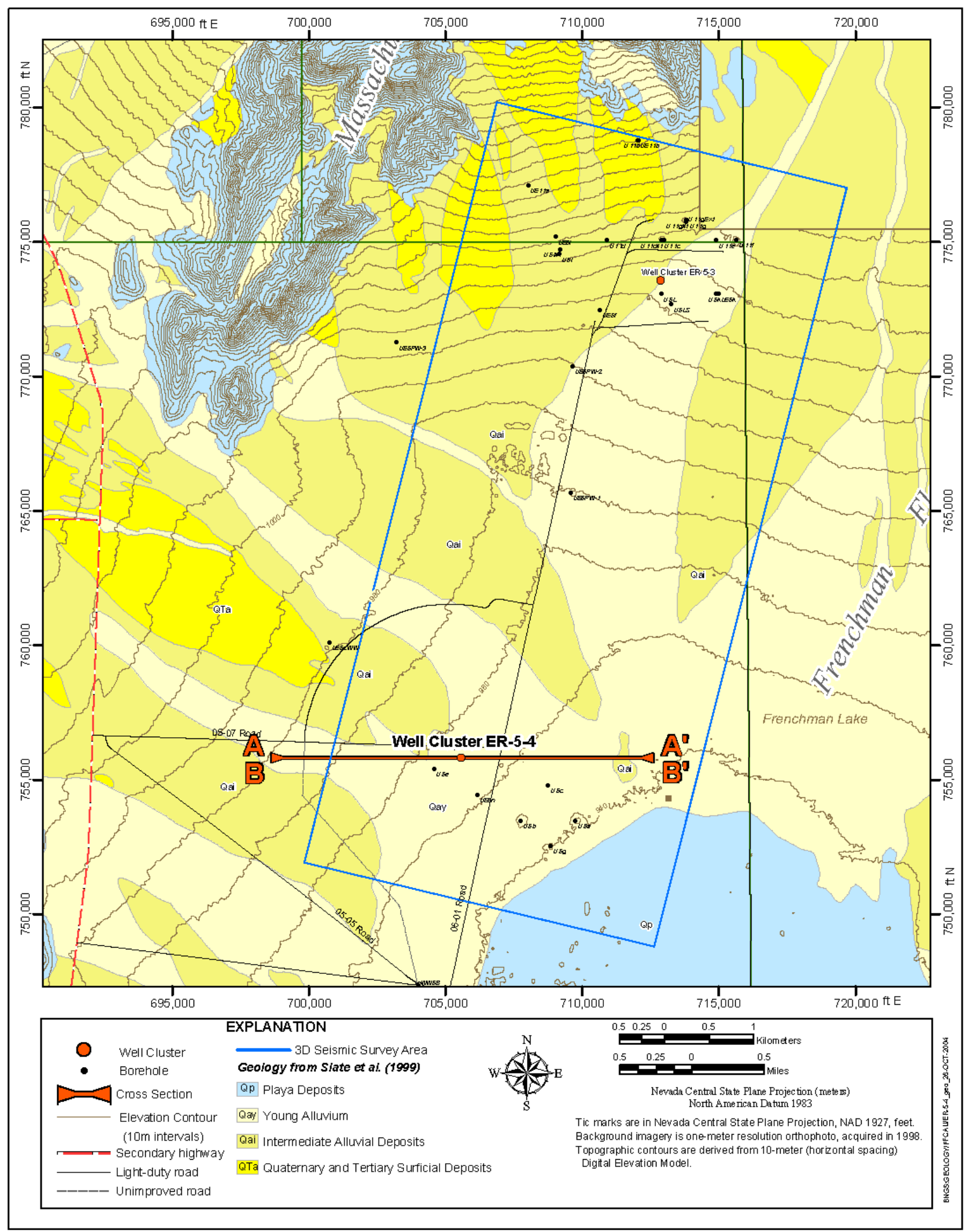

Figure 4-1

Surface Geologic Map of the Well Cluster ER-5-4 Site 
This page intentionally left blank. 
Barnes et al., 1982). Volcanic rocks exposed in the highlands around Frenchman Flat are mostly Miocene-age tuffs of generally rhyolitic composition erupted from large calderas located $40 \mathrm{~km}(25 \mathrm{mi})$ northwest of Frenchman Flat, and intermediate-composition tuffs, lavas, and debris flows from the Wahmonie volcanic center located adjacent to Frenchman Flat on the west. Tuffaceous sedimentary rocks appear to occur within a rather narrow, linear, northeast-trending depositional area that generally corresponds to the topographic axis of the basin (Prothro and Drellack, 1997). These rocks are exposed along the southern margin of the basin where they consist of a diverse assemblage of fluvial and lacustrine sandstone and mudrocks, freshwater limestone, conglomerate, and volcanic tuff. The tuffaceous sedimentary rocks appear to be partly coeval with the older volcanic rocks and thus likely interfinger with the volcanic rocks beneath Frenchman Flat. Paleozoic-age sedimentary rocks are exposed along the south and east sides of Frenchman Flat and consist mostly of carbonate rocks ranging in age from Cambrian to Mississippian. Drilling and geophysical data from Frenchman Flat indicate that many of the formations exposed along the margins of the basin are present beneath Frenchman Flat and have been buried by thick aprons of alluvial debris shed from the exposed highlands during basin development. Alluvial deposits obtain a thickness of $1,676.4 \mathrm{~m}(5,500 \mathrm{ft})$ in the central portion of Frenchman Flat based on interpretation of seismic data (Prothro, 2002).

Modeling of surface gravity data shows the basin to be a northeast-trending, roughly oval, bowl-shaped depression (Grauch and Hudson, 1995; Phelps and Graham, 2002). Using the gravity inversion method, Phelps and Graham (2002) estimated the maximum depth to Paleozoic rocks beneath the Frenchman Flat basin at 2,400 $\mathrm{m}(8,000 \mathrm{ft})$. Recently acquired 3-D seismic reflection data from a 36.3-square-kilometer (14-square-mile) area of northern and central Frenchman Flat (Figure 4-1) indicate a maximum depth of about 3,017.5 $\mathrm{m}(9,900 \mathrm{ft})$ within the seismic survey area corresponding to the central portion of the basin (Great Basin Exploration Consultants [GBEC], 2002).

Although the gravity data do not indicate any major horst-and-graben structures beneath the basin, analysis of aeromagnetic data from Frenchman Flat reveals magnetic boundaries beneath Frenchman Flat that have been interpreted to represent buried faults. Integrating the geophysical data with regional structural analyses, Grauch and Hudson (1995) developed a conceptual structural model for Frenchman Flat. This model indicates that in a broad sense Frenchman Flat is probably best described structurally as an easttilted half-graben with one or more major basin-forming faults along its eastern margin. The main faults beneath the basin are likely north-trending, down-to-the-west normal faults that merge southward into the east-northeast striking Rock Valley strike-slip fault system. 
In summary, geological and geophysical data suggest that Frenchman Flat is an oval, bowl-shaped depression elongated in a northeast direction. The basin can be characterized as a large east-tilted halfgraben bounded along its eastern margin by a west-dipping normal fault(s) that likely merges into the Rock Valley strike-slip fault system beneath the southern portion of the basin. In its deepest portions, Frenchman Flat basin probably contains from 2,400 to 3,017.5 m (8,000 to 9,900 ft) of mostly Tertiaryage alluvium, volcanic rocks, and tuffaceous sedimentary rocks overlying complexly deformed Paleozoicage sedimentary rocks.

\subsubsection{Stratigraphy and Lithology}

This section describes the stratigraphic and lithologic units encountered in Wells ER-5-4 and ER-5-4\#2. The descriptions are based on the detailed lithologic logs provided in Appendix C. The stratigraphic and lithologic units penetrated at Well Cluster ER-5-4 are illustrated in Figure 4-2.

\subsubsection{Well ER-5-4}

Well ER-5-4 penetrated 1,118.6 m (3,670 ft) of Tertiary- and Quaternary-age alluvium consisting mostly of silty to gravelly sand associated with alluvial fan deposition. Beds of finer-grained sediments (silt and clay), possibly associated with an earlier period of playa lake deposition, were penetrated from 704.7 to $896.1 \mathrm{~m}(2,312$ to $2,940 \mathrm{ft})$. A layer of volcanic ash is present from 823.6 to $825.1 \mathrm{~m}(2,702$ to $2,707 \mathrm{ft})$ within the interval of silt and clay beds. The source of the ash layer was probably the Black Mountain volcanic center (Warren et al., 2002) located approximately $80 \mathrm{~km}$ (50 mi) northwest of Frenchman Flat. Volcanic rocks associated with the Black Mountain caldera have been determined to be about 9.4 million years old (Sawyer et al., 1994). Detailed chemical, mineralogic, and micrographic analyses of sidewall core samples from Well ER-5-4 indicate that the alluvium penetrated by the well has not been significantly altered since deposition (Warren et al., 2002).

Volcanic rocks consisting of vitric to devitrified, nonwelded to partially welded ash-flow tuff were encountered at approximately 1,118.6 m (3,670 ft) in Well ER-5-4. These rocks are assigned to the Ammonia Tanks Tuff based on the abundance of felsic phenocrysts of feldspar and quartz, moderate amounts of biotite, and especially the presence of sphene and adularescent sanidine, both of which are highly diagnostic of the Ammonia Tanks Tuff. The Ammonia Tanks Tuff was erupted 11.45 million years ago (Ma) (Sawyer et al., 1994) from the Timber Mountain caldera complex located approximately 


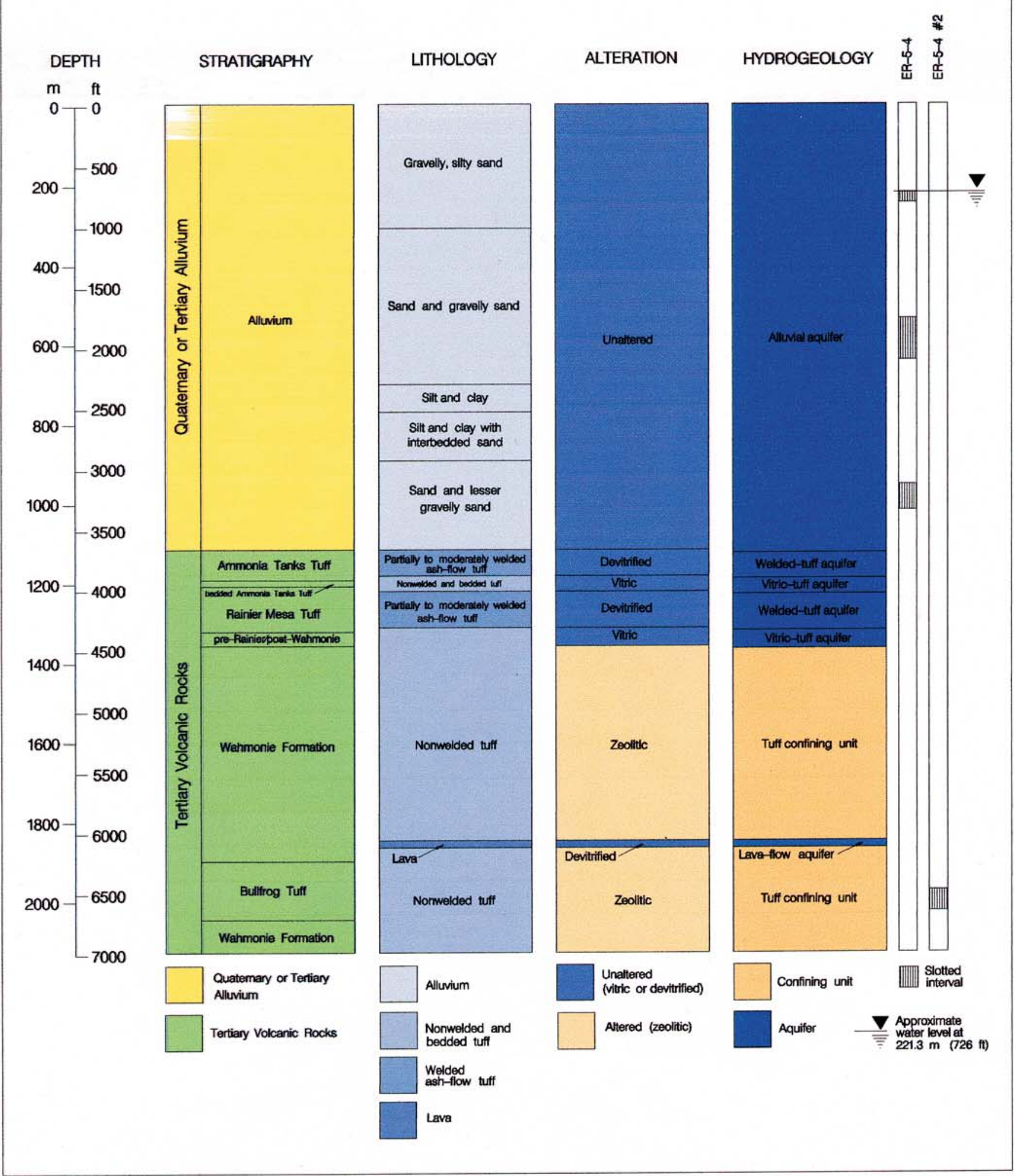

Figure 4-2

Geology and Hydrogeology of Well Cluster ER-5-4 
This page intentionally left blank. 
$40 \mathrm{~km}$ (25 mi) northwest of Frenchman Flat. Well ER-5-4 reached TD at 1,137.5 m (3,732 ft) within the Ammonia Tanks Tuff after penetrating approximately $18.8 \mathrm{~m}(62 \mathrm{ft})$ of the formation.

\subsubsection{Well ER-5-4\#2}

Well ER-5-4\#2 penetrated through the alluvium and reached TD within Tertiary volcanic rocks at a depth of 2,133.6 $\mathrm{m}(7,000 \mathrm{ft})$. Because only drill cuttings were collected during the drilling of the alluvial section at Well ER-5-4\#2 (no geophysical log data or sidewall cores were collected), detailed descriptions of the alluvium like those developed for Well ER-5-4 (Appendix C-1) are not possible. However, the close proximity of the 2 holes and an examination of the alluvium drill cuttings samples from Well ER-5-4\#2 indicate that the alluvial section penetrated by this well is very similar to that penetrated by Well ER-5-4,

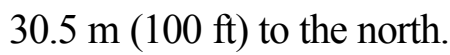

Well ER-5-4\#2 encountered volcanic rocks at a depth of 1,120.4 m (3,676 ft). The first volcanic unit encountered is the Ammonia Tanks Tuff, consisting of $76.8 \mathrm{~m}(252 \mathrm{ft})$ of mostly devitrified, partially to moderately welded ash-flow tuff (this unit was identified on the basis of mineralogy, as described in section 4.2.2.1). Below the welded Ammonia Tanks Tuff the borehole penetrated $15.8 \mathrm{~m}(52 \mathrm{ft})$ of vitric bedded tuff assigned to the bedded Ammonia Tanks Tuff. The stratigraphic assignment is based on the lithologic character of the interval and its stratigraphic position between the ash-flow tuffs of the Ammonia Tanks Tuff and Rainier Mesa Tuff.

The borehole encountered the Rainier Mesa Tuff directly beneath the bedded Ammonia Tanks Tuff, at a depth of 1,213.1 m (3,980 ft). At Well ER-5-4\#2, the Rainier Mesa Tuff consists of $114.6 \mathrm{~m}$ (376 ft) of vitric to devitrified nonwelded to moderately welded ash-flow tuff. The stratigraphic assignment of Rainier Mesa Tuff to the rocks penetrated from 1,213.1 to $1,327.7 \mathrm{~m}(3,980$ to $4,356 \mathrm{ft})$ is based on the ash-flow tuff lithology, stratigraphic position below the Ammonia Tanks Tuff, and the primary mineralogy, which consists of moderate amounts of quartz, feldspar, and biotite, only rare volcanic lithic fragments, and no sphene. The Rainier Mesa Tuff was erupted 11.6 Ma (Sawyer et. al., 1994) from the Timber Mountain caldera complex.

Many of the drill cuttings samples collected below the Rainier Mesa Tuff are of poor quality, with many samples containing substantial amounts of material caved from higher intervals. In addition, caving of the borehole wall, along with large borehole wash-outs and zones of lost circulation, disrupted the circulating fluid in the borehole and caused considerable mixing of the drill cuttings suspended in the fluid column, further degrading the quality of the samples. Although of poor quality, the samples do provide general 
information on the rocks encountered by the well. Incorporating additional information from sidewall core samples, geophysical logs, laboratory analyses, surrounding geologic exposures, and regional stratigraphic concepts allows for reasonably confident stratigraphic determinations and lithologic descriptions of the units penetrated by the well.

Below the Rainier Mesa Tuff, the well appears to have penetrated $35.4 \mathrm{~m}$ (116 ft) of nonwelded and bedded tuffs in the depth interval 1,327.7 to 1,363.1 m (4,356 to 4,472 ft). Because of the very poor quality of the drill cuttings samples from this interval, the rocks are assigned as an informal grouping referred to as pre-Rainier Mesa Tuff / post-Wahmonie Formation, undifferentiated. This interval is likely to include formations such as the tuff of Holmes Road, Topopah Spring Tuff, and Calico Hills Formation. Although difficult to determine due to the poor quality of the samples, the interval appears to be unaltered (i.e., vitric), based on a comparison of resistivity values from the interval with those from overlying vitric intervals and underlying zeolitic intervals (see Appendix D for geophysical log plots). The base of the interval is constrained by a sidewall core taken at 1,366.4 $\mathrm{m}(4,483 \mathrm{ft})$ that was identified as Wahmonie Formation, and is picked at an abrupt decrease in natural gamma radiation of the rock observed on the gamma ray $\log$ at $1,363.1 \mathrm{~m}(4,472 \mathrm{ft})$.

Below the depth of $1,363.1 \mathrm{~m}(4,472 \mathrm{ft})$, the well penetrated $540.7 \mathrm{~m}$ (1,774 ft) of mostly zeolitic nonwelded tuff assigned to the Wahmonie Formation. Drill cuttings samples are of very poor quality above $1,554.5 \mathrm{~m}(5,100 \mathrm{ft})$ due to severe contamination by material caving in from higher intervals. Below $1,554.5 \mathrm{~m}(5,100 \mathrm{ft})$ the quality of the samples improves due to a significant reduction of caved material as a result of casing being set at 1,477.9 $\mathrm{m}(4,848.8 \mathrm{ft})$ prior to drilling below $1,573.7 \mathrm{~m}(5,163 \mathrm{ft})$, and thus eliminating the sources of post-Wahmonie Formation contamination. The assignment of these rocks to the Wahmonie Formation is based mainly on the high abundance of mafic minerals (particularly orthopyroxene), the absence of quartz observed in the drilling cuttings samples below 1,554.5 m $(5,100 \mathrm{ft})$, and interpretation of two sidewall cores obtained from the depths of $1,366.4$ and $1,413 \mathrm{~m}$ (4,483 and 4,639 ft). The abundance of orthopyroxene is characteristic of the upper portions of the Wahmonie Formation. Drill cuttings from the sample interval 1,514.9 to $1,517.9 \mathrm{~m}$ (4,970 to 4,980 ft) were tentatively identified by mineralogical analysis as Calico Hills Formation (WoldeGabriel et al., 2003). The samples may represent material caved from higher intervals, or indicate that the Calico Hills Formation is intercalated with the upper portions of the Wahmonie Formation. The relatively low resistivity of the formation as observed on the dual laterolog (Appendix D) is characteristic of zeolitic, poorly welded tuffs, and is consistent with the lithology of the two sidewall core samples taken from the interval and the majority of the samples below $1,554.5 \mathrm{~m}(5,100 \mathrm{ft})$. An interval of lava was tentatively identified in the 
Wahmonie Formation, in the interval $1,850.7$ to $1,869.6 \mathrm{~m}(6,072$ to $6,134 \mathrm{ft})$, based mainly on a conspicuous abrupt increase in resistivity and density through this interval. Rocks of the Wahmonie Formation were erupted 13.0 Ma (Sawyer et. al., 1994) from the Wahmonie volcano, the remnants of which form the highlands that border Frenchman Flat on the west.

The Bullfrog Tuff was encountered below the Wahmonie Formation, from 1,903.8 to 2,051.3 m (6,246 to $6,730 \mathrm{ft}$ ). The unit consists of $147.5 \mathrm{~m}$ (484 ft) of zeolitic nonwelded tuff. The assignment of this interval to the Bullfrog Tuff is based mainly on the presence of moderate amounts of felsic phenocrysts (including quartz), and biotite. Drill cuttings from the sample interval 1,898.9 to 1,902.0 m (6,230 to 6,240 ft) were identified by petrographic and microprobe analyses as the genetically related Prow Pass Tuff (WoldeGabriel et al., 2003), indicating that the Prow Pass Tuff occurs at the top of the interval. The Bullfrog Tuff was erupted 13.25 Ma (Sawyer et. al., 1994) from the Silent Canyon caldera complex located approximately $56 \mathrm{~km}(35 \mathrm{mi})$ northwest of Frenchman Flat.

The natural gamma ray spectroscopy log (Appendix D) indicates possible fractures within the Bullfrog Tuff at 1,932.1, 1,953.8, 1,970.2, and 1,980.9 $\mathrm{m}(6,339,6,410,6,464$, and 6,499 ft). However, the lack of corresponding breakouts on the caliper log and no increase in water production or lost circulation while drilling at these depths suggest that the fractures are probably healed. Conspicuous breakouts are observed on the caliper log between the depths of 1,998.0 and 2,024.5 m (6,555 to 6,642 ft). An increase in water production and lost circulation occurred during drilling of this interval, suggesting that these fractures are open. Preliminary analysis of the field print of the Electric-Micro Imager® $\log$ indicates that these fractures strike almost due north and dip more than 70 degrees to both the west and east.

Another interval of Wahmonie Formation was penetrated below the Bullfrog Tuff from the depth of $2,051.3 \mathrm{~m}(6,730 \mathrm{ft})$ to the TD of the well at 2,133.6 $\mathrm{m}(7,000 \mathrm{ft})$. This interval of Wahmonie also appears to consist mainly of zeolitic nonwelded tuff. However, no orthopyroxene was observed, which suggests the interval is part of the lower portion of the Wahmonie, possibly the Salyer Member which has only rare orthopyroxene. The occurrence of Wahmonie Formation stratigraphically above and below the Bullfrog Tuff indicates that the 2 formations, originating from different sources, interfinger beneath Frenchman Flat, and that the lower portions of the Wahmonie Formation are older than 13.25 Ma. 


\subsubsection{Alteration}

Although the alluvium contains volcanic clasts that are altered (i.e., zeolitic) the alluvial matrix is primarily vitric, indicating that the alluvium has undergone very little in situ alteration since deposition (Warren et al., 2002).

The volcanic rocks above the Wahmonie Formation (depth interval 1,120.4 to 1,363.1 $\mathrm{m}[3,676$ to 4,472 ft]), including the Ammonia Tanks and Rainier Mesa Tuffs, are also primarily unaltered. The nonwelded and bedded tuffs are vitric, while the more welded ash-flow tuffs are devitrified (Figure 4-2).

The rocks assigned to the Wahmonie Formation and Bullfrog Tuff are pervasively zeolitic. Quantitative mineralogic analysis shows that the degree of alteration increases with depth (WoldeGabriel et al., 2003), as summarized here and illustrated on Figure 4-2. However, the absence of analcime, the fresh unaltered appearance of the biotite, and the lack of any apparent alteration of feldspar phenocrysts suggest that this alteration is typical zeolitization and not the more advanced quartzo-feldspathic alteration present in volcanic rocks near the bottom of the Tertiary section in Yucca Flat and at the base of the Bullfrog Tuff immediately above the top of the Paleozoic rocks in Well ER-5-3\#2 in northern Frenchman Flat. This may indicate that the base of the Tertiary volcanic rock section (i.e., top of Paleozoic sedimentary rocks) in Well ER-5-4\#2 is still some distance below the TD of the well.

In summary, rocks from the surface to $1,363.1 \mathrm{~m}(4,472 \mathrm{ft})$ at Well Cluster ER-5-4 are primarily unaltered, while rocks from 1,363.1 to 2,133.6 m (4,472 to 7,000 ft) show pervasive zeolitic alteration, with the degree of alteration increasing with depth.

\subsection{Predicted Versus Actual Geology}

Figure 4-3 provides a comparison of the geology predicted to be encountered by the two wells of the cluster prior to drilling with the geology actually encountered by the wells. Originally, Well ER-5-4 was planned to be drilled completely through the alluvium and reach a TD of $1,066.8 \mathrm{~m}(3,500 \mathrm{ft})$ in the underlying volcanic rocks. However, drilling was terminated due to severe borehole instability while apparently still in alluvium, at a depth of 1,137.5 m (3,732 ft). Later detailed examination of the cuttings and comparison of data from Well ER-5-4\#2 indicated that the top of the volcanic rocks was encountered at approximately $1,118.6 \mathrm{~m}(3,670 \mathrm{ft})$ in Well ER-5-4. The predicted depth to the top of the volcanic rocks was $1,005.8 \mathrm{~m}(3,300 \mathrm{ft})$, indicating that the alluvium is $112.8 \mathrm{~m}(370 \mathrm{ft})$ thicker than originally predicted. 

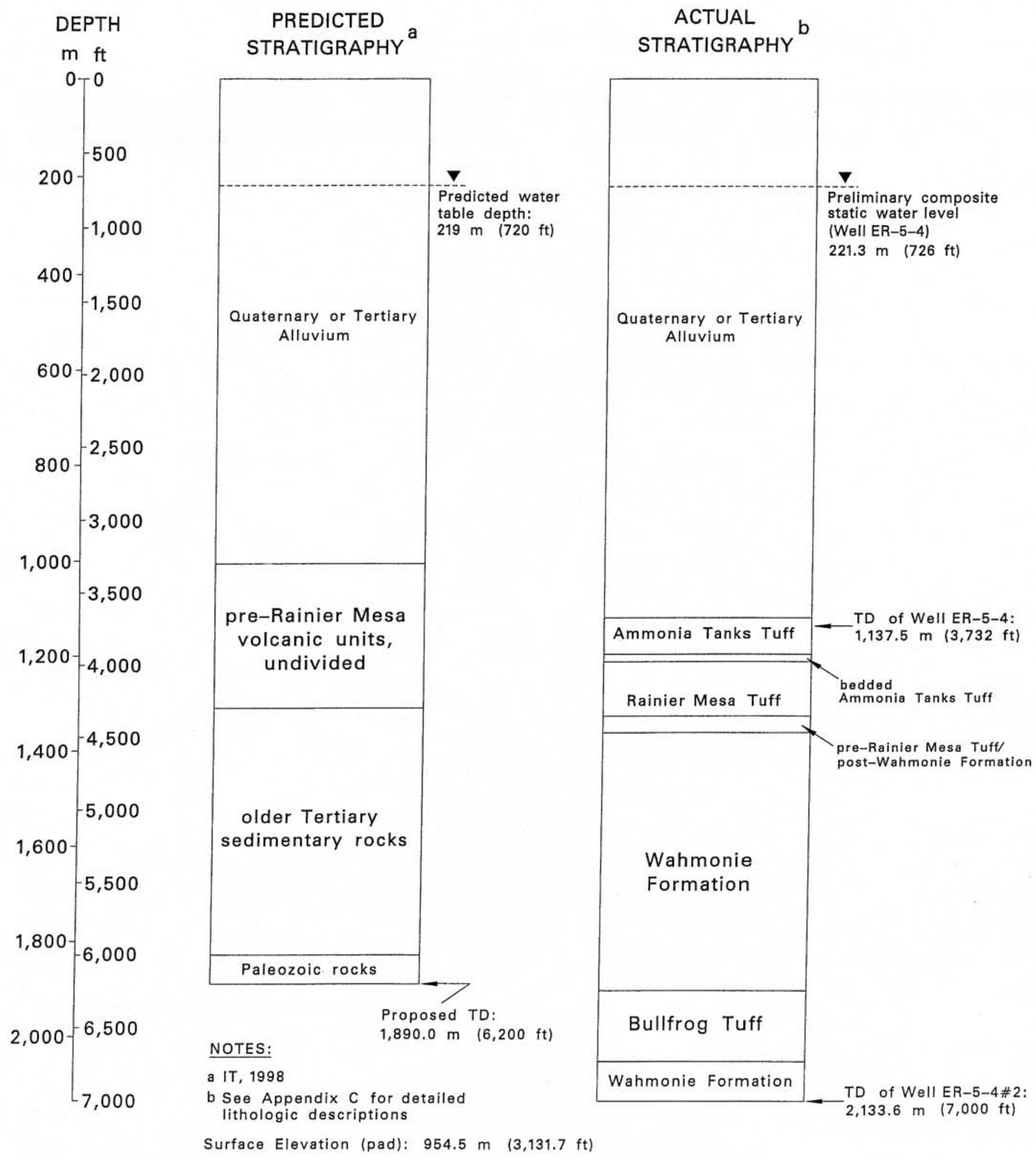

Figure 4-3

Predicted and Actual Stratigraphy at Well Cluster ER-5-4 
Well ER-5-4\#2 was drilled $30.5 \mathrm{~m}$ (100 ft) south of Well ER-5-4. It encountered the top of the volcanic

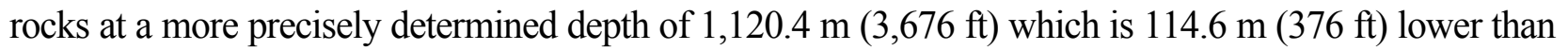
the pre-drill prediction for the top of volcanic rocks. It was predicted that the first volcanic rocks encountered would be poorly welded, zeolitic tuffs older than the Rainier Mesa Tuff. However, after penetrating the alluvium, the well encountered $207.3 \mathrm{~m}$ (680 ft) of mostly welded ash-flow tuff assigned to the Rainier Mesa and younger Ammonia Tanks Tuffs, indicating that the ash-flow tuffs of the Timber Mountain Group extend farther south beneath Frenchman Flat than previously modeled (IT, 1998). The rocks were also primarily unaltered (vitric and devitrified), which indicates that the top of pervasive zeolitic alteration is deeper than predicted. The well was predicted to penetrate approximately $304.8 \mathrm{~m}(1,000 \mathrm{ft})$ of pre-Rainier Mesa Tuff units consisting of zeolitic, nonwelded and bedded tuffs, then cut approximately $518.2 \mathrm{~m}(1,700 \mathrm{ft})$ of older Tertiary sedimentary rocks, before encountering Paleozoic rocks at 1,828.8 $\mathrm{m}$ $(6,000 \mathrm{ft})$. At the depth of 2,133.6 m (7,000 ft) Well ER-5-4\#2 was still in pre-Rainier Mesa volcanic rocks after penetrating $805.9 \mathrm{~m}(2,644 \mathrm{ft})$ of these rocks. This indicates that these rocks are more than $501.1 \mathrm{~m}(1,644 \mathrm{ft})$ thicker than predicted. The well reached TD in Wahmonie Formation and thus did not reach the older Tertiary sedimentary rocks or Paleozoic rocks.

Analysis of seismic data in combination with information from the well cluster indicates that the top of the Paleozoic rocks is probably no shallower than 2,377.4 $\mathrm{m}$ (7,800 ft) (Prothro, 2002), and thus at least $548.6 \mathrm{~m}(1,800 \mathrm{ft})$ deeper at the well cluster site than originally predicted.

Cross sections constructed through the well site prior to drilling show a series of down-to-the-west normal faults located both east and west of the site (IT, 2001a). These faults were interpreted to be major basinforming faults, forming a series of north-trending, east-tilted half-grabens. The locations of the faults were based primarily on interpretation of aeromagnetic data (Grauch and Hudson, 1995). However reinterpretation of the gravity data (Phelps and Graham, 2002) and recently acquired and interpreted 3-D seismic reflection data indicate that no major faults are present in the vicinity of the well cluster site, as shown on the west-east seismic profile in Figure 4-4. Figure 4-5, a west-east geologic cross section through the well site, also shows no major faults in this area. The cross section incorporates information from the wells and from an interpretation of the 3-D seismic reflection data (Prothro, 2002). 


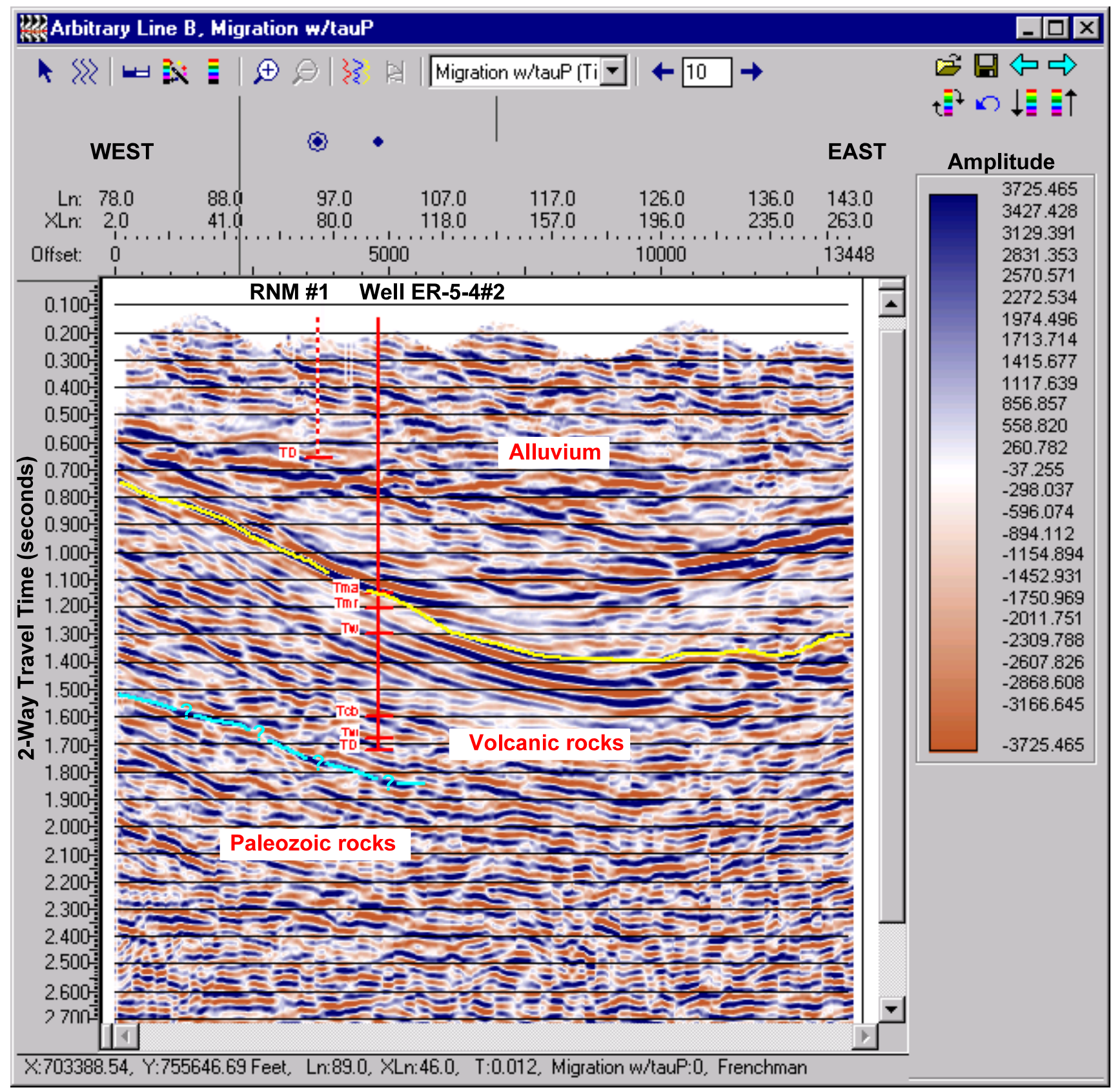

Figure 4-4

West-East Seismic Profile through Well ER-5-4\#2 
This page intentionally left blank. 


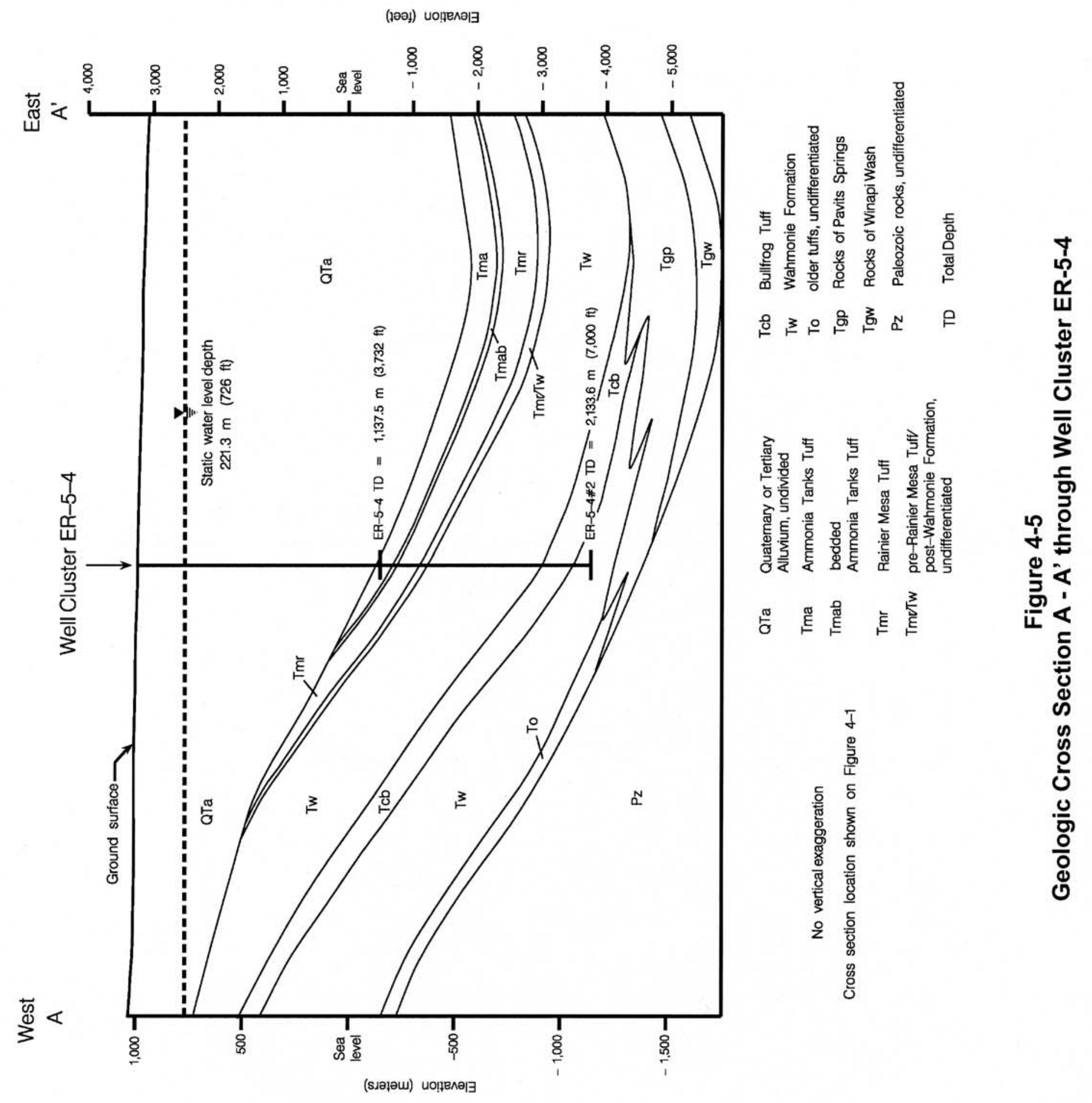




\subsection{Hydrogeology}

Figure 4-6 shows a west-east cross sectional view of the distribution of hydrogeologic units encountered at the well cluster. The poorly consolidated and unaltered alluvium encountered in both wells forms an alluvial aquifer (Figure 4-2), which is 1,120 m (3,676 ft) thick in Well ER-5-4\#2. The preliminary composite, static water levels measured in both wells soon after completion are within the alluvial aquifer. Finer grained sediments that may represent playa deposits, encountered from 704.7 to $896.1 \mathrm{~m} \mathrm{(2,312} \mathrm{to}$ 2,940 ft) in Well ER-5-4, are likely less porous and permeable than the more typical alluvial deposits, forming an interval within the alluvial aquifer that may actually behave hydrologically more like a confining unit.

Below the alluvial aquifer, the 2 intervals of partially to moderately welded ash-flow tuff assigned to the Ammonia Tanks and Rainier Mesa Tuffs form welded-tuff aquifers. The unaltered, bedded Ammonia Tanks Tuff present between the 2 welded tuff aquifers forms a thin (18.1 m [52 ft] interval of vitric-tuff aquifer that, at least locally, separates the 2 welded-tuff aquifers. The vitric, nonwelded base of the Rainier Mesa Tuff and the nonwelded and bedded tuffs present between the Rainier Mesa Tuff and the Wahmonie Formation form another interval of vitric-tuff aquifer that is $46.3 \mathrm{~m}(152 \mathrm{ft})$ thick.

The top of pervasive zeolitization that appears to correspond generally to the top of the Wahmonie Formation forms the upper contact of a very thick section of tuff confining unit. This confining unit is present in the interval from $1,363.1 \mathrm{~m}(4,472 \mathrm{ft})$ to the TD of Well ER-5-4\#2 at 2,133.6 m (7,000 ft), and consists of zeolitic, nonwelded tuffs of the Wahmonie Formation and Bullfrog Tuff. Only a relatively thin (18.9 $\mathrm{m}$ [62 ft]) interval of lava that forms a lava-flow aquifer breaks up this thick monotonous sequence of tuff confining unit. These rocks are considered to be a confining unit based on lithology and alteration, which was generally confirmed by the lack of significant water production during drilling. However, fractures encountered within the Bullfrog Tuff in the interval 1998.0 to 2,024.5 m (6,555 to 6,642 ft) appear to have produced significant amounts water during drilling (see Section 3.4.2). Although it is not unusual for tuff confining units to have open fractures that produce water, production is usually short-lived because the fractures typically are poorly developed and do not form systematic, interconnecting networks that can sustain water production. Well development and testing activities at Well ER-5-4\#2 will provide additional information on the productivity of these fractures. 

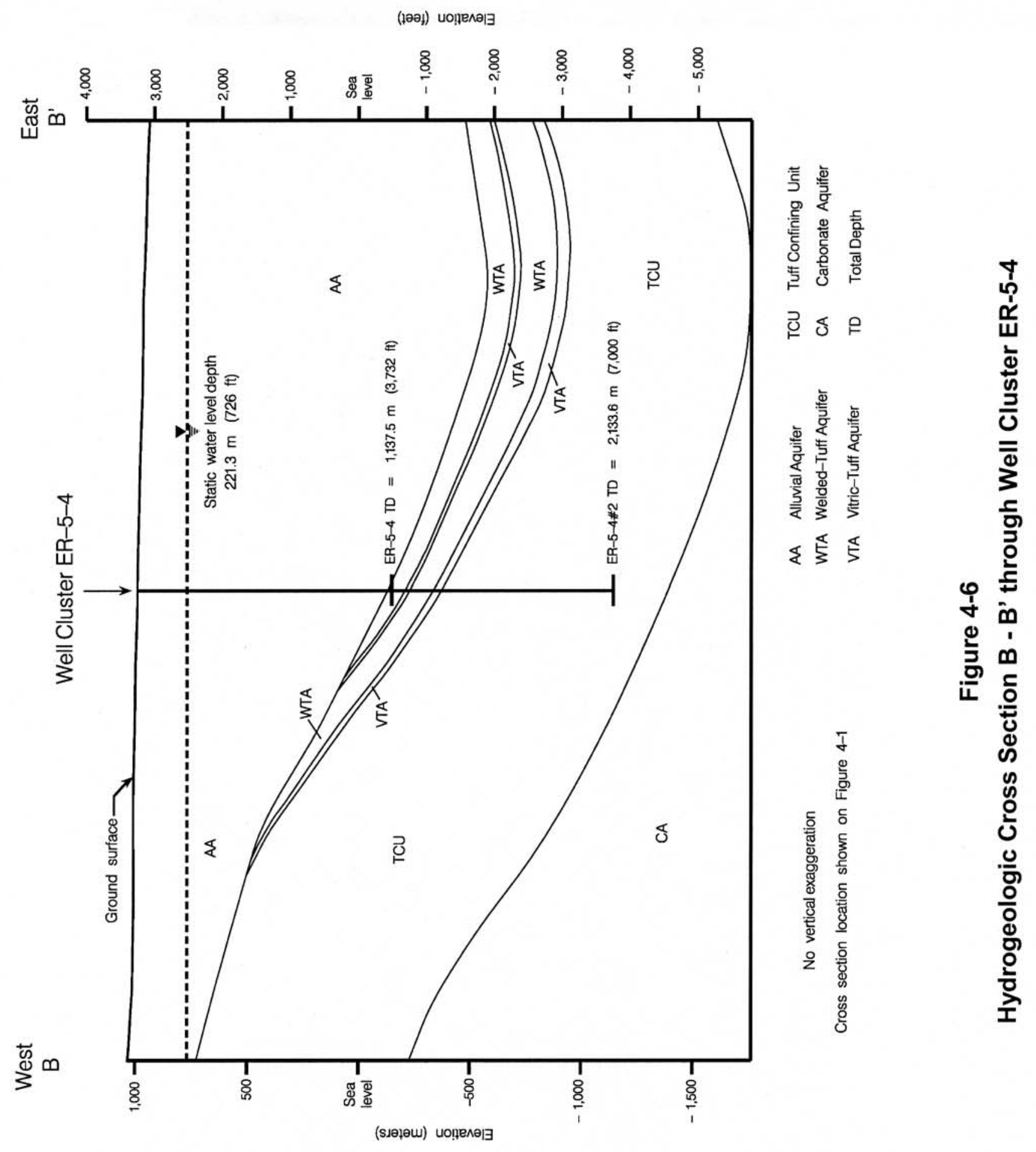
This page intentionally left blank. 


\subsection{Summary, Recommendations, and Lessons Learned}

\subsection{Summary}

Well Cluster ER-5-4 consists of two wells, ER-5-4 and ER-5-4 \#2, drilled $30.5 \mathrm{~m}$ (100 ft) apart in the central portion of Frenchman Flat of the NTS. The wells were drilled as part of the hydrogeologic investigation well program for Frenchman Flat. The primary purpose of constructing Well Cluster ER-5-4 was to obtain information that will help characterize the hydrogeology near a group of underground nuclear test locations in this part of Frenchman Flat. Data from these wells will allow for more accurate modeling of groundwater flow and radionuclide migration in the region.

Drilling operations at Well Cluster ER-5-4 were hampered by persistent problems related to borehole instability and lost circulation that resulted in numerous delays and required additional equipment and engineering designs to mitigate and resolve. Drilling of Well ER-5-4, the first well of the cluster, commenced on February 11, 2001, and concluded on March 18, 2001, at a depth of 1,137.5 m $(3,732 \mathrm{ft})$. The completion design of the well allows for access to groundwater in the alluvial aquifer at three discrete locations within the well bore. A piezometer tube slotted from 220.2 to $247.4 \mathrm{~m}$ (722.6 to $811.7 \mathrm{ft}$ ) was installed at the water level depth, and a string of 51/2-in. stainless-steel casing was installed with slotted intervals from 539.5 to $644.2 \mathrm{~m}(1,770.0$ to $2,113.4 \mathrm{ft})$ and 955.9 to $1,021.1 \mathrm{~m}(3,136.6$ to $3,350.1 \mathrm{ft})$.

Drilling at Well ER-5-4\#2 began on July 16, 2002, and concluded on September 11, 2002, at a depth of 2,133.6 $\mathrm{m}(7,000 \mathrm{ft})$. The well completion consists of a string of 51/2-in. stainless-steel casing slotted from $1,976.9$ to $2,029.3 \mathrm{~m}(6,486.0$ to $6,657.7 \mathrm{ft})$, open to the tuff confining unit, with no gravel or cement.

Geologic data collected at Well Cluster ER-5-4 consist of drill cuttings collected every $3 \mathrm{~m}$ (10 ft) from below the conductor casing to total depth in both wells. In addition, a total of 156 sidewall core samples was collected from both holes. Numerous geophysical logs were also run in each hole to verify the geology, determine hydrologic characteristics, and aid in well construction.

The geology encountered at the well cluster consists, in descending order, of 1,120.4 m (3,676 ft) of Quaternary and Tertiary alluvium, $242.6 \mathrm{~m}$ (796 ft) of generally unaltered nonwelded and welded ash-flow tuff of Tertiary age, and $770.5 \mathrm{~m}$ (2,528 ft) of mostly zeolitic nonwelded tuff, also of Tertiary age. The preliminary, composite water level in Well ER-5-4 is approximately $221.3 \mathrm{~m}$ (726 ft) below ground surface. No radionuclides above background were encountered in the groundwater produced from either 
well. Preliminary (field-monitoring) data indicated no lead above permitted levels for dissolved lead in the drilling effluent.

\subsection{Recommendations}

All geologic, geophysical, and hydrologic information, including results from well development activities, from Well Cluster ER-5-4 should be used to refine hydrogeologic models of Frenchman Flat.

\subsection{Lessons Learned}

The alluvium in the central portion of Frenchman Flat can be difficult to drill through due to the poorly consolidated nature of the unit that can cause significant problems associated with borehole wall instability. Sloughing and washout of the borehole wall and loss of fluid circulation can severely hamper drilling operations. The use of bentonite drilling mud and lost circulation material can help alleviate some of these problems but may not be sufficient to successfully drill through the alluvial section. Additional drilling methods such as extra casing strings and cement plugs may be necessary for successful drilling of this unit. 


\subsection{References}

Barnes, H., E. B. Ekren, C. L. Rodgers, and D. S. Hedlund, 1982. "Geology and Tectonic Maps for the Mercury Quadrangle, Nye and Clark Counties, Nevada," U.S. Geological Survey, Miscellaneous Geologic Investigations Series Map I-1197, scale 1:24,000.

Bechtel Nevada, 2001. UGTA Project Health and Safety Plan. January 2001. Las Vegas, NV.

Bechtel Nevada, 2001. Main-Hole Drilling and Completion of Underground Test Area (UGTA)

Investigation Well ER-5-4. Field Activity Work Plan Number D-002-001.01. February 5, 2001. Las Vegas, NV.

Bechtel Nevada, 2002. Main-Hole Drilling and Completion of Underground Test Area (UGTA) Investigation Well ER-5-4 \#2. Field Activity Work Plan Number D-006-001.02. June 20, 2002. Las Vegas, NV.

BN, see Bechtel Nevada.

DOE/NV, see U.S. Department of Energy, Nevada Operations Office.

GBEC, see Great Basin Exploration Consultants.

Grauch, V. J. S., and M. R. Hudson, 1995. Written communication prepared for DOE/NV. Subject: "Preliminary Analysis of Major Structures and Lithologic Boundaries for the Frenchman Flat Model Area.” U.S. Geological Survey, Denver, CO.

Great Basin Exploration Consultants, 2002. Written communication prepared for Bechtel Nevada, Las Vegas, NV. Subject: "Frenchman Flat 3D Seismic Survey, ER-5-4\#2 Synthetic Seismogram, TimeDepth Curve, and Correlation with seismic Data, Nevada Test Site, Nevada," August 16, 2002. Lakewood, CO.

Hinrichs, E. N., 1968. "Geologic Map of the Camp Desert Rock Quadrangle, Nye County, Nevada," U.S. Geological Survey Geologic Quadrangle Map G-726, scale 1:24,000.

Hinrichs, E. N., and E. J. McKay, 1965. "Geologic Map of the Plutonium Valley Quadrangle, Nye and Lincoln Counties, Nevada,” U.S. Geological Survey Geologic Quadrangle Map 384, scale 1:24,000.

IT, see IT Corporation.

IT Corporation, 1998. Corrective Action Unit 98: Frenchman Flat Data Analysis Task, Volume I: Hydrostratigraphic Model Documentation Package. August 1998. DOE/NV/13052-044. 
IT Corporation, 2000. Frenchman Flat Hydrogeologic Investigation Wells Drilling and Completion Criteria. February 2000, Rev. No. 0. ITLV/13052-094. Las Vegas, NV.

IT Corporation, 2001a. Addendum to the Frenchman Flat Hydrogeologic Investigation Wells Drilling and Completion Criteria. January 2001, Rev. No. 1. ITLV/13052-094-REV.1-ADD. Las Vegas, NV.

IT Corporation, 2001b. Written communication prepared for DOE/NV. Subject: "Frenchman Flat ER-5-4 Well Data Report," September 2001. Las Vegas, NV.

IT Corporation, 2003. Written communication prepared for NNSA/NSO. Subject: "Frenchman Flat ER-5-4\#2 Well Data Report,” March 2003. Las Vegas, NV.

Liebendorfer, P.J., Nevada Division of Environmental Protection, 2000. Letter to R.C. Wycoff (DOE) entitled, "U.S. Department of Energy's Request for Approval to Drill and a Waiver to the Fluid Management Plan (FMP) for Underground Test Area (UGTA) Wells ER-5-3 and ER-5-4, January 25, 2000, at Corrective Action Unit 98 Frenchman Flat." Carson City, NV.

McKeown, F. A., D. L. Healey, and C. H. Miller, 1976. "Geologic Map of the Yucca Lake Quadrangle, Nye County, Nevada,” U.S. Geological Survey Geologic Quadrangle Map GQ-1327, scale 1:24,000.

Phelps, G. A., and S. E. Graham, 2002. "Preliminary Gravity Inversion Model of Frenchman Flat Basin, Nevada Test Site," U.S. Geological Survey Open-File Report 02-363.

Poole, F. G., 1965. "Geologic Map of the Frenchman Flat Quadrangle, Nye and Lincoln Counties, Nevada,” U.S. Geological Survey Geologic Quadrangle Map GC-456, scale 1:24,000.

Poole, F. G., D. P. Elston, and W. J. Carr, 1965. "Geologic Map of the Cane Spring Quadrangle, Nye County, Nevada,” U.S. Geological Survey Geologic Quadrangle Map GQ-455, scale 1:24,000.

Prothro, L. B., Bechtel Nevada, 2002. Written communication prepared for NNSA/NSO, Las Vegas, NV. Subject: "Geologic Interpretation of the Frenchman Flat 3-D Seismic Data, Summary Report." August 2002. Las Vegas, NV.

Prothro, L. B., and S. L. Drellack, Jr., 1997. Review and Reconnaissance of the Hydrogeology of Tertiary Sedimentary Rocks in the Vicinity of Frenchman Flat, Nevada Test Site. DOE/NV/11718--155. Las Vegas, NV.

Sawyer, D. A., R. J. Fleck, M. A. Lanphere, R. G. Warren, and D. E. Broxton, 1994. Episodic Caldera Volcanism in the Miocene Southwest Nevada Volcanic Field: Revised Stratigraphic Caldera Framework, ${ }^{40} \mathrm{Ar} /{ }^{39} \mathrm{Ar}$ Geochronology and Implications for Magmatism and Extension. Geological Society of America Bulletin, v. 67, n. 10, pp. 1,304-1,318. 
Slate, J. L., M. E. Berry, P. D. Rowley, C. J. Fridrich, K. S. Morgan, J. B. Workman, O. D. Young, G. L. Dixon, V. S. Williams, E. H. McKee, D. A. Ponce, T. G. Hildenbrand, WC Swadley, S. C. Lundstrom, E. B. Ekren, R. G. Warren, J. C. Cole, R. J. Fleck, M. A. Lanphere, D. A. Sawyer, S. A. Minor, D. J. Grunwald, R. J. Laczniak, C. M. Menges, J. C. Yount, and A. S. Jayko, 1999. Digital Geologic Map of the Nevada Test Site and Vicinity, Nye, Lincoln, and Clark Counties, Nevada, and Inyo County, California. U.S. Geological Survey Open-File Report 99-554-A, scale 1:120,000.

U.S. Department of Energy, Nevada Operations Office, 1996. "Underground Test Area Subproject Waste Management Plan," Rev. 1, DOE/NV--343. Las Vegas, NV.

U.S. Department of Energy, Nevada Operations Office, 1998. Nevada Environmental Restoration Project Health and Safety Plan, Revision 3, February 1998. Las Vegas, NV.

U.S. Department of Energy, Nevada Operations Office, 1999. "Fluid Management Plan for the Underground Test Area Project," DOE/NV--370, Rev. 2. In Underground Test Area Subproject Waste Management Plan, Rev. 1, DOE/NV--343. Las Vegas, NV.

Warren, R. G., F. C. Benedict Jr., T. P. Rose, D. K. Smith, S. J. Chipera, E. C. Kluk, and K. M. Raven, 2002. "Alluvial Layering and Distribution of Reactive Phases within Drill Holes ER5/4 and UE5n of Frenchman Flat. Los Alamos National Laboratory Report LA-UR-02-6206. Los Alamos, NM.

WoldeGrabriel, G., S. Chipera, G. Keating, E. Kluk, S. Levy, and P. Snow, Los Alamos National Laboratory, 2003. Personal communication to L. B. Prothro (Bechtel Nevada). Subject: "Preliminary Report on Geological Characterization of Well ER-5-4 \#2, Frenchman Flat, Nevada Test Site.” Los Alamos National Laboratory Report in progress. 
This page intentionally left blank. 


\section{Appendix A \\ Drilling Data}

A-1 Drilling Parameter Logs for Well Cluster ER-5-4

A-2 Casing and Tubing Data for Well Cluster ER-5-4

A-3 Well Cluster ER-5-4 Drilling Fluids and Cement Composition 


\section{Appendix A-1 \\ Drilling Parameter Logs for Well Cluster ER-5-4}




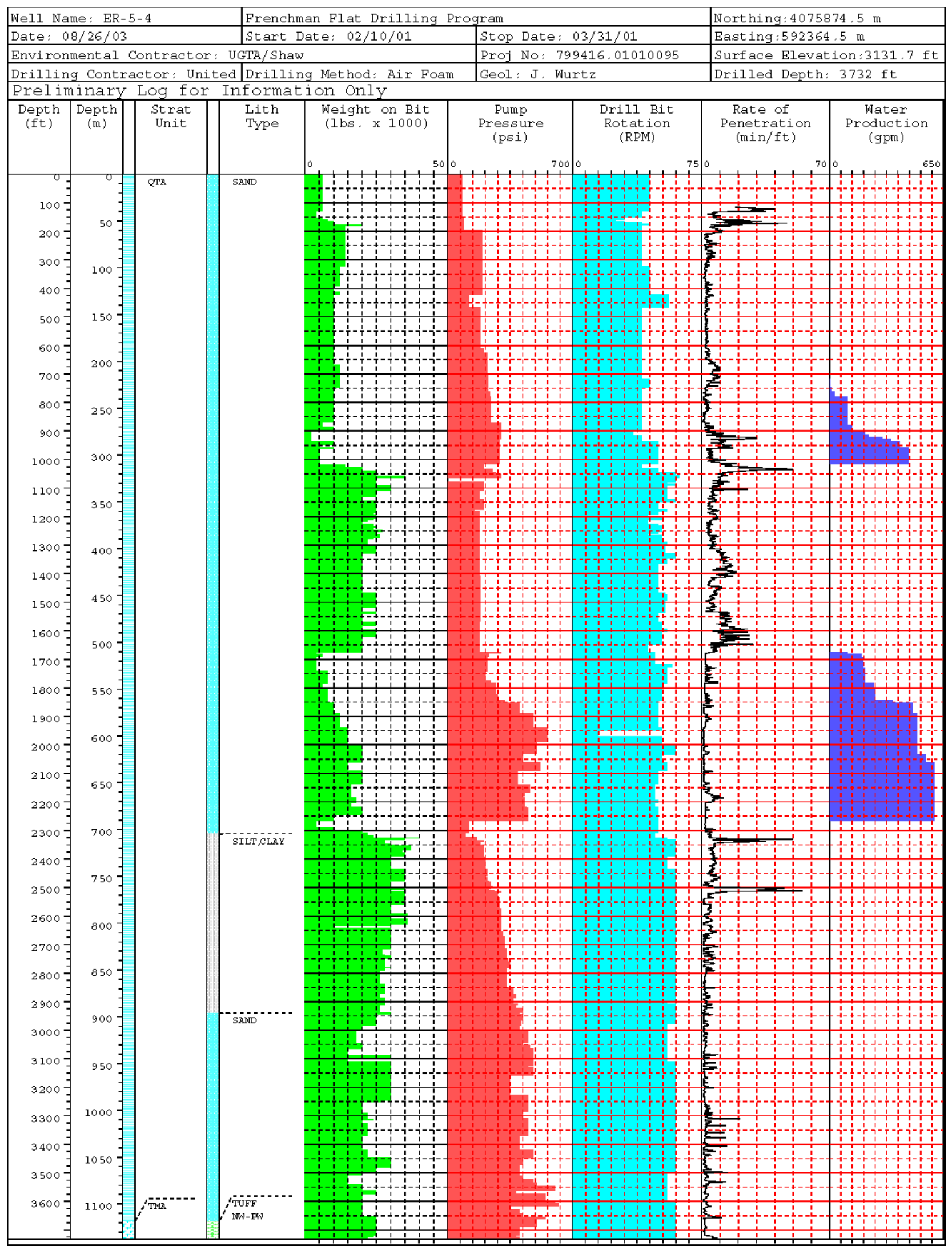




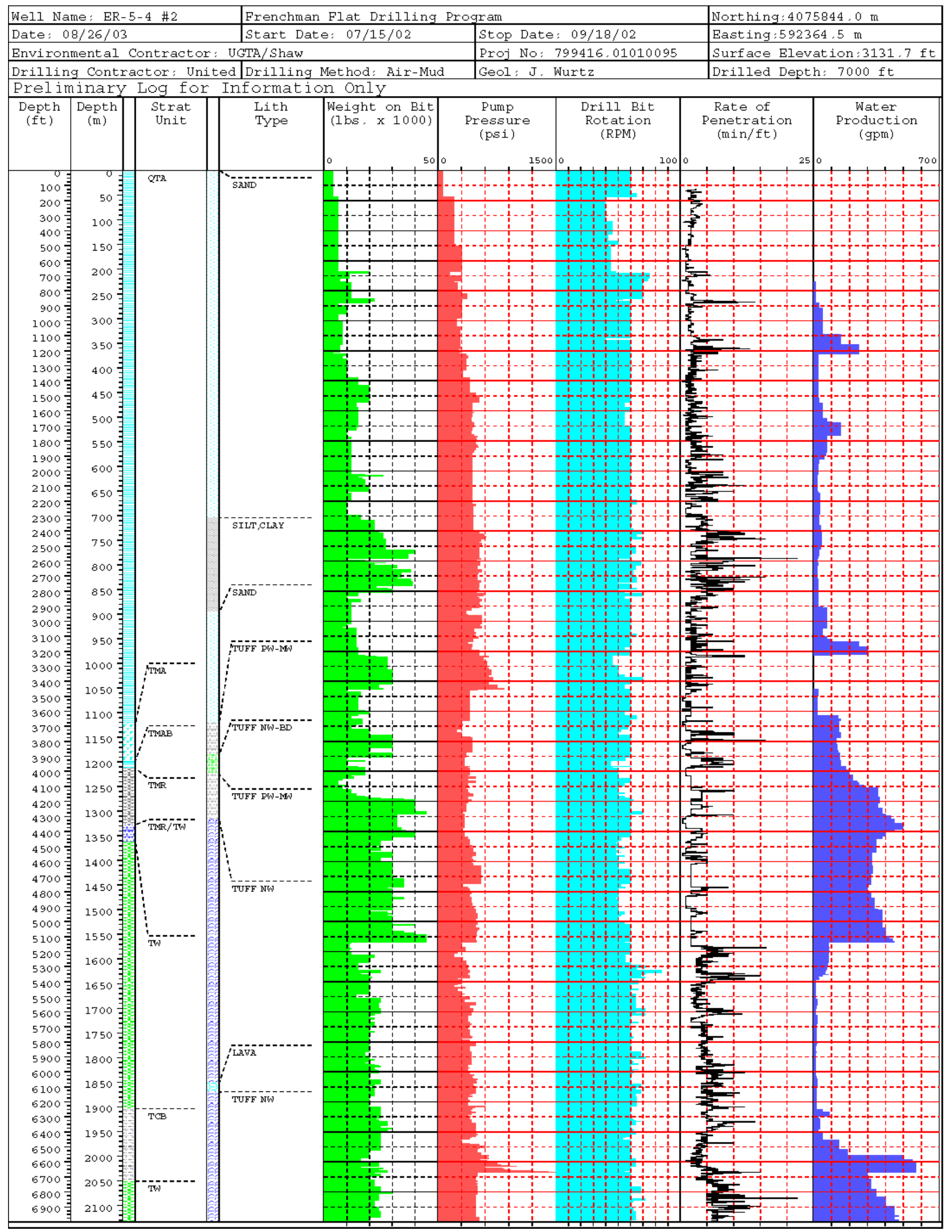


Appendix A-2

Casing and Tubing Data for Well Cluster ER-5-4 
Table A-2-1

Casing and Tubing Data for Well ER-5-4

\begin{tabular}{|c|c|c|c|c|c|c|c|}
\hline Casing & $\begin{array}{l}\text { Depth Interval } \\
\text { meters } \\
\text { (feet) }\end{array}$ & Type & Grade & $\begin{array}{l}\text { Outside } \\
\text { Diameter } \\
\text { centimeters } \\
\text { (inches) }\end{array}$ & $\begin{array}{c}\text { Inside } \\
\text { Diameter } \\
\text { centimeters } \\
\text { (inches) }\end{array}$ & $\begin{array}{c}\text { Wall } \\
\text { Thickness } \\
\text { centimeters } \\
\text { (inches) }\end{array}$ & $\begin{array}{l}\text { Weight } \\
\text { per foot } \\
\text { (pounds) }\end{array}$ \\
\hline $\begin{array}{l}\text { Conductor } \\
\text { Casing }\end{array}$ & $\begin{array}{c}0 \text { to } 36.3 \\
(0 \text { to } 119.0)\end{array}$ & $\begin{array}{l}\text { Carbon Steel } \\
\text { PE Weld }\end{array}$ & $\mathrm{N} / \mathrm{A}$ & $\begin{array}{l}76.2 \\
(30)\end{array}$ & $\begin{array}{l}74.295 \\
(29.250)\end{array}$ & $\begin{array}{c}0.953 \\
(0.375)\end{array}$ & 118.65 \\
\hline Surface Casing & $\begin{array}{l}0 \text { to } 279.1 \\
\text { ( } 0 \text { to } 915.5)\end{array}$ & $\begin{array}{l}\text { Carbon } \\
\text { Steel }\end{array}$ & K55 & $\begin{array}{c}50.80 \\
(20)\end{array}$ & $\begin{array}{l}48.575 \\
(19.124)\end{array}$ & $\begin{array}{l}1.113 \\
(0.438)\end{array}$ & 94.0 \\
\hline \multirow{2}{*}{$\begin{array}{l}\text { Intermediate } \\
\text { Casing }\end{array}$} & $\begin{array}{c}0 \text { to } 63.9 \\
(0 \text { to } 209.6)\end{array}$ & Carbon Steel & K55 & $\begin{array}{c}33.97 \\
(13.375)\end{array}$ & $\begin{array}{l}31.788 \\
(12.515)\end{array}$ & $\begin{array}{c}1.092 \\
(0.430)\end{array}$ & 61.0 \\
\hline & $\begin{array}{c}63.9 \text { to } 510.0 \\
\text { (209.6 to } 1,673.3)\end{array}$ & Carbon Steel & K55 & $\begin{array}{c}33.97 \\
(13.375)\end{array}$ & $\begin{array}{c}32.042 \\
(12.615)\end{array}$ & $\begin{array}{c}0.965 \\
(0.380)\end{array}$ & 54.5 \\
\hline Piezometer String & $\begin{array}{c}0 \text { to } 248.1 \\
\text { (1 to } 813.9)\end{array}$ & Stainless Steel & P110 & $\begin{array}{l}7.303 \\
(2.875)\end{array}$ & $\begin{array}{c}6.200 \\
(2.441)\end{array}$ & $\begin{array}{c}0.551 \\
(0.217)\end{array}$ & 6.5 \\
\hline $\begin{array}{l}\text { Completion Casing } \\
\text { (with cross-over) }\end{array}$ & $\begin{array}{c}0 \text { to } 500.2 \\
(0 \text { to } 1,641.0)\end{array}$ & $\begin{array}{l}\text { Carbon Steel } \\
\text { with internal } \\
\text { epoxy coating }\end{array}$ & N80 & $\begin{array}{l}19.37 \\
(7.625)\end{array}$ & $\begin{array}{l}17.701 \\
(6.969)\end{array}$ & $\begin{array}{c}0.833 \\
(0.328)\end{array}$ & 26.4 \\
\hline Completion Casing & $\begin{array}{c}500.2 \text { to } 1,048.0 \\
(1,641.0 \text { to } 3,438.3)\end{array}$ & Stainless Steel & SSTP304 & $\begin{array}{c}14.13 \\
(5.563)\end{array}$ & $\begin{array}{l}12.819 \\
(5.047) \\
\end{array}$ & $\begin{array}{c}0.655 \\
(0.258) \\
\end{array}$ & 14.6 \\
\hline
\end{tabular}

Table A-2-2

Casing and Tubing Data for Well ER-5-4\#2

\begin{tabular}{|c|c|c|c|c|c|c|c|}
\hline Casing & $\begin{array}{l}\text { Depth Interval } \\
\text { meters } \\
\text { (feet) }\end{array}$ & Type & Grade & $\begin{array}{l}\text { Outside } \\
\text { Diameter } \\
\text { centimeters } \\
\text { (inches) }\end{array}$ & $\begin{array}{c}\text { Inside } \\
\text { Diameter } \\
\text { centimeters } \\
\text { (inches) }\end{array}$ & $\begin{array}{c}\text { Wall } \\
\text { Thickness } \\
\text { centimeters } \\
\text { (inches) }\end{array}$ & $\begin{array}{l}\text { Weight } \\
\text { per foot } \\
\text { pounds }\end{array}$ \\
\hline $\begin{array}{l}\text { Conductor } \\
\text { Casing }\end{array}$ & $\begin{array}{l}0 \text { to } 33.8 \\
(0 \text { to } 111)\end{array}$ & $\begin{array}{l}\text { Carbon } \\
\text { Steel } \\
\text { PE Weld }\end{array}$ & $\mathrm{N} / \mathrm{A}$ & $\begin{array}{l}76.2 \\
(30)\end{array}$ & $\begin{array}{c}74.3 \\
(29.250)\end{array}$ & $\begin{array}{c}0.9 \\
(0.375)\end{array}$ & 118.65 \\
\hline $\begin{array}{l}\text { Surface } \\
\text { Casing }\end{array}$ & $\begin{array}{l}0 \text { to } 266.1 \\
(0 \text { to } 873)\end{array}$ & \multirow{4}{*}{$\begin{array}{l}\text { Carbon } \\
\text { Steel }\end{array}$} & \multirow{4}{*}{ K55 } & $\begin{array}{l}50.8 \\
(20)\end{array}$ & $\begin{array}{c}48.6 \\
(19.124)\end{array}$ & $\begin{array}{c}2.2 \\
(0.876)\end{array}$ & 94 \\
\hline \multirow{3}{*}{$\begin{array}{l}\text { Intermediate } \\
\text { Casing }\end{array}$} & $\begin{array}{c}0 \text { to } 280.2 \\
(0 \text { to } 919.2)\end{array}$ & & & $\begin{array}{c}34.0 \\
(13.375)\end{array}$ & $\begin{array}{c}31.8 \\
(12.515)\end{array}$ & $\begin{array}{c}2.2 \\
(0.865)\end{array}$ & 61 \\
\hline & $\begin{array}{l}280.2 \text { to } 965.9 \\
(919.2 \text { to } 3,169)\end{array}$ & & & $\begin{array}{c}34.0 \\
(13.375)\end{array}$ & $\begin{array}{c}32.0 \\
(12.615)\end{array}$ & $\begin{array}{c}1.9 \\
(0.760)\end{array}$ & 54.5 \\
\hline & $\begin{array}{c}0 \text { to } 1,477.9 \\
(0 \text { to } 4,848.8)\end{array}$ & & & $\begin{array}{c}24.4 \\
(9.625)\end{array}$ & $\begin{array}{c}22.7 \\
(8.921)\end{array}$ & $\begin{array}{c}1.8 \\
(0.704)\end{array}$ & 36 \\
\hline $\begin{array}{l}\text { Completion } \\
\text { Casing }\end{array}$ & $\begin{array}{c}1,437.1-2,030.0 \\
(4,715-6,660)\end{array}$ & $\begin{array}{l}\text { Stainless } \\
\text { Steel }\end{array}$ & SSTP304 & $\begin{array}{c}14.1 \\
(5.563)\end{array}$ & $\begin{array}{c}12.8 \\
(5.047)\end{array}$ & $\begin{array}{c}1.3 \\
(0.516)\end{array}$ & 14.6 \\
\hline
\end{tabular}


This page intentionally left blank.

A-2-2 
Appendix A-3

Well Cluster ER-5-4 Drilling Fluids and Cement Composition 
Table A-3-1

Well ER-5-4 Drilling Fluids

\begin{tabular}{|c|c|}
\hline Typical Air-Foam/Polymer Mix & Typical Bentonite Mud Mix \\
\hline $\begin{array}{c}26.5 \text { liters (7 gallons) Geofoam }{ }^{\circledR a} \\
7.6 \text { liters (2 gallons) LP- } 701^{\circledR a} \\
\text { per } \\
7,949 \text { liters ( } 50 \text { barrels) water }\end{array}$ & $\begin{array}{c}\begin{array}{c}50 \text { viscosity Geo Gel } \\
\text { and LP- } 701^{\circledR} \text { mixture }\end{array} \\
\text { Walnut hulls, cotton seed pellets, and Prima Seal } \\
\text { as lost circulation material added periodically }\end{array}$ \\
\hline
\end{tabular}

a $\quad$ Geofoam ${ }^{\circledR}$ foaming agent, LP- $701{ }^{\circledR}$ polymer additive, Geo Gel ${ }^{\circledR}$ bentonite mud and Prima Seal ${ }^{\circledR}$ lost circulation material are products of Geo Drilling Fluids, Inc.

\section{NOTES:}

1. All water used to mix drilling fluids for Well ER-5-4 came from a fill stands at the Radioactive Waste Management Site in Area 5 and Mercury, Nevada in Area 23.

2. A concentrated solution of lithium bromide was added to all introduced fluids to make up a final concentration of approximately 15 to 20 milligrams per liter.

\section{Table A-3-2 \\ Well ER-5-4\#2 Drilling Fluids}

\begin{tabular}{|c|c|}
\hline Typical Air-Foam/Polymer Mix & Typical Bentonite Mud Mix \\
\hline $\begin{array}{l}22.7 \text { to } 30.3 \text { liters ( } 6 \text { to } 8 \text { gallons) Geofoam }{ }^{\circledR a} \\
\begin{array}{c}7.6 \text { liters ( } 2 \text { gallons) LP }-701^{\circledR a} \\
\text { per } \\
7,949 \text { liters ( } 50 \text { barrels) water }\end{array}\end{array}$ & $\begin{array}{c}50 \text { to } 60 \text { viscosity } \mathrm{Geo} \mathrm{Gel}^{\circledR \mathrm{a}} \\
\text { and LP-701 }{ }^{\circledR} \text { mixture } \\
\text { Cedar fiber and Prima Seal }{ }^{\circledR} \text { as lost circulation } \\
\text { material added periodically above } 1,039.4 \mathrm{~m}(3,410 \mathrm{ft})\end{array}$ \\
\hline
\end{tabular}

a $\quad$ Geofoam ${ }^{\circledR}$ foaming agent, LP- $701^{\circledR}$ polymer additive, Geo Gel ${ }^{\circledR}$ bentonite mud and Prima Seal ${ }^{\circledR}$ lost circulation material are products of Geo Drilling Fluids, Inc.

\section{NOTES:}

1. All water used to mix drilling fluids for Well ER-5-4 \#2 came from a fill stand at the Radioactive Waste Management Site and Water Well 5B, both located in Area 5.

2. A concentrated solution of lithium bromide was added to all introduced fluids to make up a final concentration of approximately 20 milligrams per liter. 
Table A-3-3

Well ER-5-4 Cement Composition

\begin{tabular}{|c|c|c|c|c|}
\hline \multirow{2}{*}{$\begin{array}{l}\text { Cement } \\
\text { Composition }\end{array}$} & \multicolumn{4}{|c|}{$\begin{array}{c}\text { Cemented Interval } \\
\text { (depth below ground surface) }\end{array}$} \\
\hline & $\begin{array}{l}\text { 30-inch } \\
\text { Conductor Casing }\end{array}$ & $\begin{array}{l}\text { 20-inch } \\
\text { Surface Casing }\end{array}$ & $\begin{array}{c}\text { 13d -inch } \\
\text { Intermediate } \\
\text { Casing } \\
\end{array}$ & $\begin{array}{c}51 / 2 \text {-inch } \\
\text { Completion String }\end{array}$ \\
\hline Type II neat & $\begin{array}{l}0-37.2 \mathrm{~m}^{\mathrm{a}} \\
\left(0-122 \mathrm{ft}^{\mathrm{b}}\right)\end{array}$ & $\begin{array}{l}248.4-277.4 \mathrm{~m} \\
(815-910 \mathrm{ft})\end{array}$ & $\begin{array}{l}442.6-510.5 \mathrm{~m} \\
\left(1,452^{c}-1,675 \mathrm{ft}\right)\end{array}$ & $\begin{array}{l}499.9-522.7 \mathrm{~m} \\
(1,640-1,715 \mathrm{ft}) \\
668.1-744.9 \mathrm{~m} \\
(2,192-2,444 \mathrm{ft}) \\
855.3-890.6 \mathrm{~m} \\
(2,806-2,922 \mathrm{ft}) \\
\\
902.2-918.7 \mathrm{~m} \\
(2,960-3,014 \mathrm{ft})\end{array}$ \\
\hline
\end{tabular}

a meter(s) b foot (feet) c estimated

Table A-3-4

Well ER-5-4\#2 Cement Composition

\begin{tabular}{|c|c|c|c|c|c|}
\hline \multirow{2}{*}{$\begin{array}{c}\text { Cement } \\
\text { Composition }\end{array}$} & \multicolumn{5}{|c|}{$\begin{array}{c}\text { Cemented Interval } \\
\text { (depth below ground surface) }\end{array}$} \\
\hline & $\begin{array}{c}\text { 30-inch } \\
\text { Conductor } \\
\text { Casing }\end{array}$ & $\begin{array}{l}\text { 20-inch } \\
\text { Surface Casing }\end{array}$ & $\begin{array}{c}\text { 13d -inch } \\
\text { Intermediate } \\
\text { Casing }\end{array}$ & $\begin{array}{c}9 e-\text { inch } \\
\text { Intermediate } \\
\text { Casing }\end{array}$ & $\begin{array}{c}\text { 51/2-inch } \\
\text { Completion } \\
\text { String }\end{array}$ \\
\hline Type II neat & $\begin{array}{l}0-35.1 \mathrm{~m}^{\mathrm{a}} \\
\left(0-115 \mathrm{ft}^{\mathrm{b}}\right)\end{array}$ & $\begin{array}{c}192.9 \text { to } 266.4 \mathrm{~m} \\
(633-874 \mathrm{ft})\end{array}$ & $\begin{array}{c}944.0-966.2 \mathrm{~m} \\
\left(3,097^{\mathrm{C}}-3,170 \mathrm{ft}\right)\end{array}$ & $\begin{array}{c}1,465.8-1,478.3 \mathrm{~m} \\
\left(4,809^{c}-4,850 \mathrm{ft}\right)\end{array}$ & Not used \\
\hline
\end{tabular}

a meter(s) b foot (feet) c estimated 
Appendix B

Well Cluster ER-5-4 Fluid Management Data 


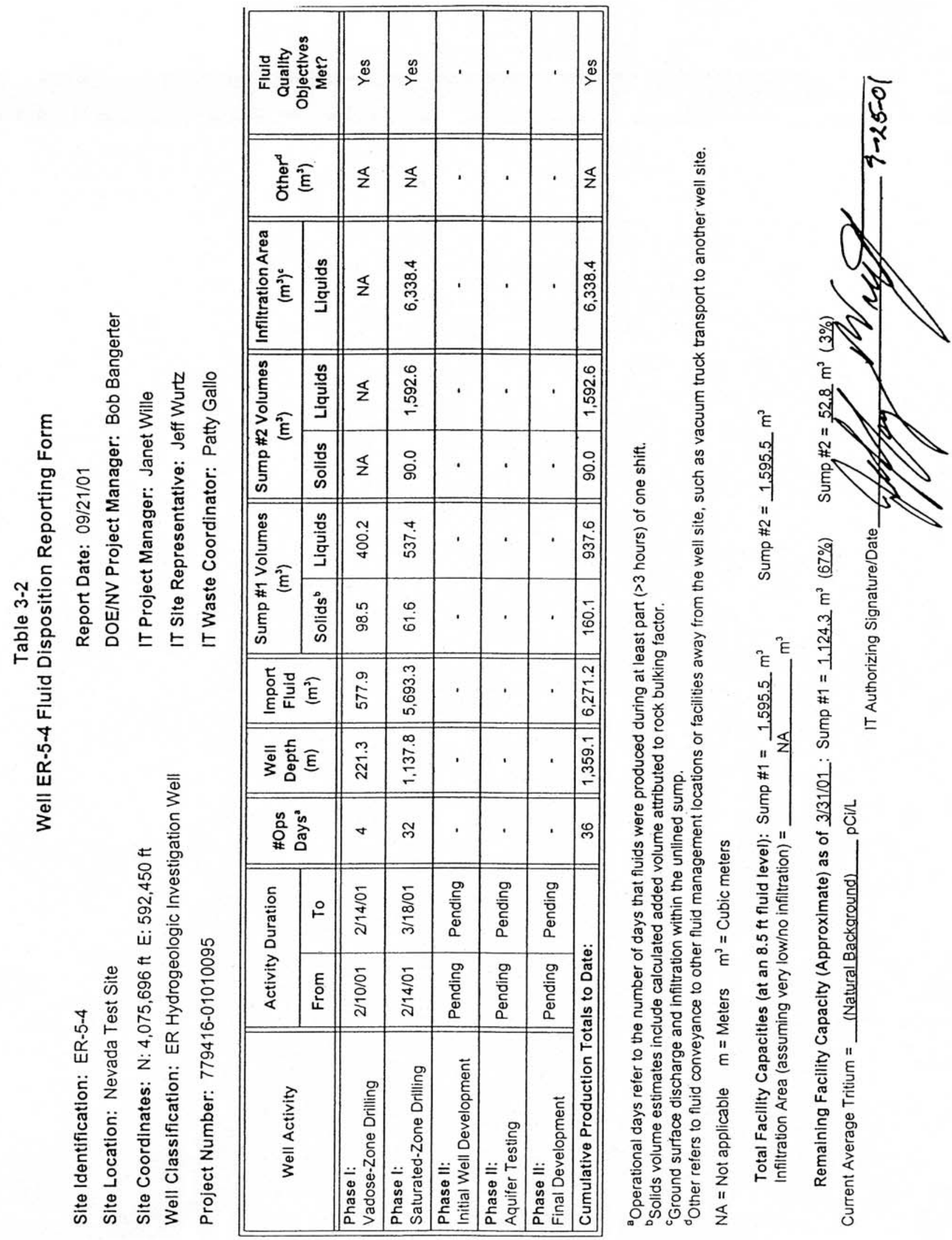




\section{Preliminary Analytical Results for Fluid Management Samples from Well ER-5-4}

\begin{tabular}{|c|c|c|c|c|c|c|c|c|c|c|c|c|c|c|}
\hline \multirow{2}{*}{$\begin{array}{c}\text { Sample } \\
\text { Number }\end{array}$} & \multirow{2}{*}{$\begin{array}{c}\text { Date \& } \\
\text { Time } \\
\text { Collected } \\
\end{array}$} & \multirow{2}{*}{ Comment } & & \multicolumn{8}{|c|}{ Resource Conservation Recovery Act Metals (mg/L) ${ }^{a}$} & \multirow{2}{*}{$\begin{array}{c}\text { Gross } \\
\text { Alpha } \\
{(\mathrm{pCi} / \mathrm{L})^{\mathrm{b}}}^{\mathrm{b}} \\
\end{array}$} & \multirow{2}{*}{$\begin{array}{c}\text { Gross } \\
\text { Beta } \\
\text { (pCi/L) }\end{array}$} & \multirow{2}{*}{$\begin{array}{l}\text { Tritium } \\
(\mathrm{pC} / \mathrm{L} / \mathrm{L})\end{array}$} \\
\hline & & & & Arsenic & Barium & Cadmium & Chromium & Lead & Selenium & Silver & Mercury & & & \\
\hline \multirow[b]{2}{*}{ ER-5-4-02141-1 } & \multirow{2}{*}{$\begin{array}{c}02 / 14 / 2001 \\
08: 50\end{array}$} & \multirow{2}{*}{$\begin{array}{l}\text { Sample taken } \\
\text { from unlined } \\
\text { Sump \#1 }\end{array}$} & Total & 0.016 & 0.38 & $0.00052(\mathrm{~B})^{\mathrm{C}}$ & 0.017 & 0.0077 & 0.0044 (B) & $0.01(U)^{d}$ & $0.0002(U)$ & & & \\
\hline & & & Dissolved & 0.0065 (B) & $0.032(B)$ & $0.005(U)$ & 0.013 & 0.003 & 0.0029 (B) & 0.00052 (B) & $0.0002(U)$ & $\begin{array}{c}5.2 \\
E^{e}=2.9\end{array}$ & $\begin{array}{c}10.4 \\
E=3.2\end{array}$ & $\begin{array}{c}150 \\
E=180(U)\end{array}$ \\
\hline \multirow{2}{*}{ ER-5-4-02141-2 } & \multirow{2}{*}{$\begin{array}{c}\text { 02/14/2001 } \\
09: 00\end{array}$} & \multirow{2}{*}{$\begin{array}{l}\text { Duplicate } \\
\text { sample taken } \\
\text { from unlined } \\
\text { Sump \#1 }\end{array}$} & Total & 0.0091 (B) & 0.16 & $0.005(\mathrm{U})$ & 0.013 & 0.0013 (B) & 0.0067 & $0.01(U)$ & $0.0002(U)$ & & & \\
\hline & & & Dissolved & 0.0071 (B) & $0.03(\mathrm{~B})$ & $0.005(U)$ & 0.012 & $0.003(\mathrm{U})$ & 0.0044 (B) & $0.01(\mathrm{U})$ & $0.0002(U)$ & $\begin{array}{c}8.5 \\
E=3.1\end{array}$ & $\begin{array}{c}12.2 \\
E=3.4\end{array}$ & $\begin{array}{c}20 \\
E=170(U)\end{array}$ \\
\hline \multirow[b]{2}{*}{ ER-5-4-03171-1 } & \multirow{2}{*}{$\begin{array}{c}03 / 17 / 2001 \\
13: 00\end{array}$} & \multirow{2}{*}{$\begin{array}{l}\text { Sample taken } \\
\text { from unlined } \\
\text { Sump \#2 }\end{array}$} & Total & 0.037 & 0.62 & $0.005(U)$ & 0.033 & 0.1 & 0.01 & $0.01(U)$ & $7.7 \mathrm{e}-05$ (B) & & & \\
\hline & & & Dissolved & 0.018 & 0.017 (B) & 0.00027 (B) & 0.014 & 0.0029 (B) & 0.0067 & 0.0016 (B) & $0.0002(U)$ & $\begin{array}{c}9.7 \\
E=2.5\end{array}$ & $\begin{array}{c}5.3 \\
E=1.8\end{array}$ & $\begin{array}{c}1,460 \\
E=270\end{array}$ \\
\hline \multirow{2}{*}{ ER-5-4-03191-1 } & \multirow{2}{*}{$\begin{array}{c}\text { 03/19/2001 } \\
12: 05\end{array}$} & \multirow{2}{*}{$\begin{array}{l}\text { Sample taken } \\
\text { from unlined } \\
\text { Sump \#1 }\end{array}$} & Total & 0.044 & 0.042 (B) & $0.005(U)$ & 0.0046 (B) & $0.003(\mathrm{U})$ & $0.005(U)$ & 0.0012 (B) & $0.0002(U)$ & & & \\
\hline & & & Dissolved & 0.045 & $0.016(B)$ & $0.005(U)$ & 0.0054 (B) & 0.00097 (B) & $0.005(U)$ & 0.0015 (B) & $0.0002(U)$ & $\begin{array}{c}7.6 \\
E=1.8\end{array}$ & $\begin{array}{c}7.2 \\
E=1.5\end{array}$ & $\begin{array}{c}250 \\
E=170(U)\end{array}$ \\
\hline \multirow[b]{2}{*}{ ER-5-4-03191-2 } & \multirow{2}{*}{$\begin{array}{c}03 / 19 / 2001 \\
12: 20\end{array}$} & \multirow{2}{*}{$\begin{array}{l}\text { Sample taken } \\
\text { from unlined } \\
\text { Sump \#2 }\end{array}$} & Total & 0.032 & 0.38 & $0.005(\mathrm{U})$ & 0.022 & 0.044 & 0.0058 & $0.01(U)$ & $9.9 \mathrm{e}-05$ (B) & & & \\
\hline & & & Dissolved & 0.016 & 0.056 (B) & $0.005(\mathrm{U})$ & $0.0082(\mathrm{~B})$ & 0.014 & 0.0047 (B) & 0.00065 (B) & $0.0002(U)$ & $\begin{array}{c}11.3 \\
E=2.7\end{array}$ & $\begin{array}{c}7.5 \\
E=2.1\end{array}$ & $\begin{array}{c}540 \\
E=190\end{array}$ \\
\hline \multicolumn{4}{|c|}{ Contract-Required Detection Limit } & 0.01 & 0.1 & 0.005 & 0.01 & 0.003 & 0.005 & 0.01 & 0.0002 & $N / A^{f}$ & $N / A$ & N/A \\
\hline \multicolumn{4}{|c|}{ Nevada Drinking Water Standard (NDWS) } & 0.05 & 2.0 & 0.005 & 0.1 & 0.015 & 0.05 & 0.1 & 0.002 & 15 & 50 & 20,000 \\
\hline & & & 5 Times NDWS & 0.25 & 10 & 0.025 & 0.5 & 0.075 & 0.25 & 0.5 & 0.01 & 75 & 250 & 100,000 \\
\hline
\end{tabular}

All analyses by Paragon Analytics, Inc.

a $\quad \mathrm{mg} / \mathrm{L}=$ milligrams per liter

b $\quad \mathrm{pCi} / \mathrm{L}=$ picocuries per liter

c $\quad B=$ Result less than Contract-Required Detection Limit, but greater than the Instrument Detection Limit

d $\quad U=$ Result less the Instrument Detection Limit or the Minimum Detectable Concentration

e $\quad E=$ Error

f N/A $=$ Not applicable 


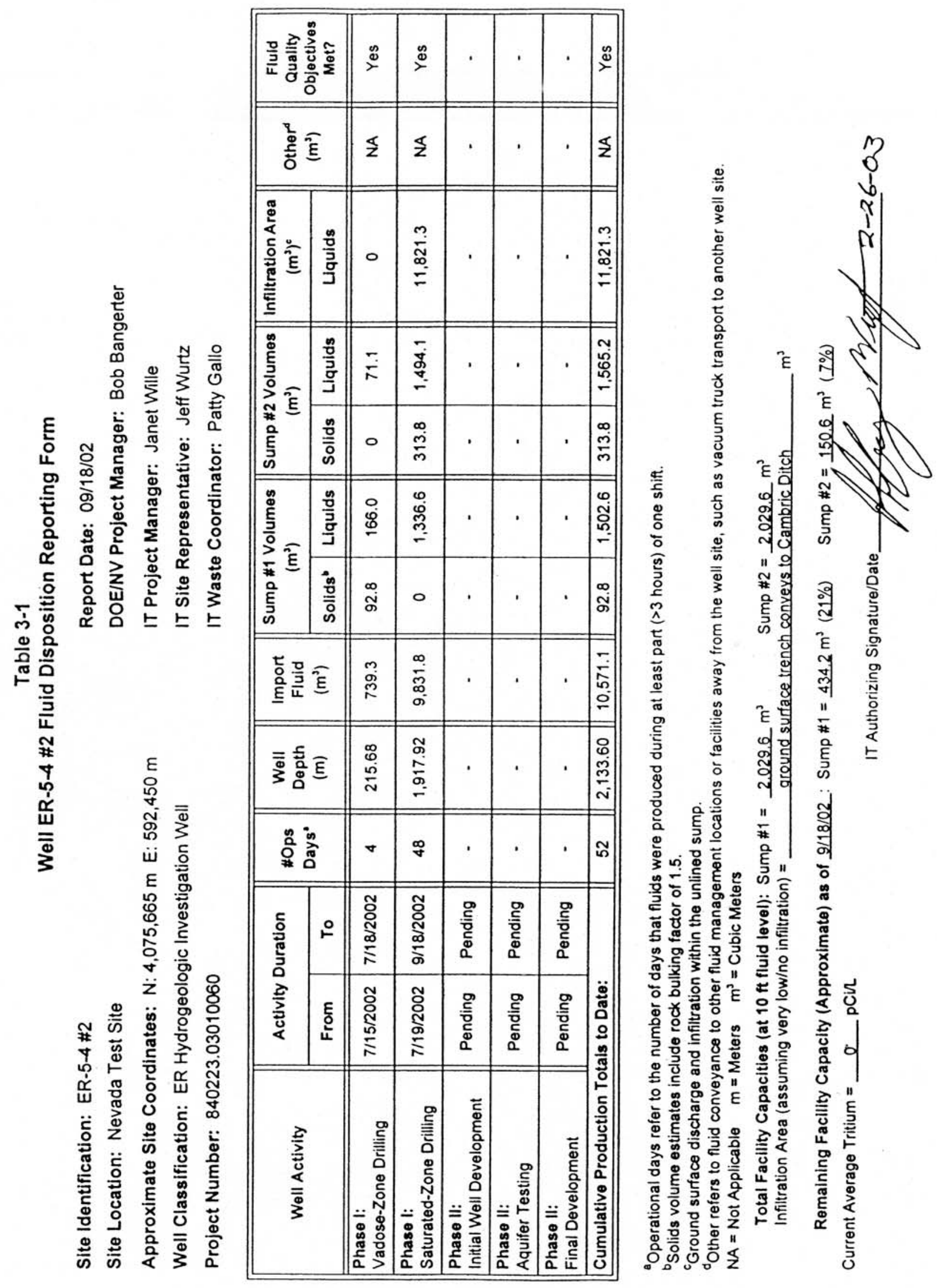


This page intentionally left blank.

B-4 


\section{Appendix C \\ Lithologic Logs for Well Cluster ER-5-4 \\ C-1 Detailed Lithologic Log for Well ER-5-4 \\ C-2 Detailed Lithologic Log for Well ER-5-4\#2}




\section{Appendix C-1}

Detailed Lithologic Log for Well ER-5-4 
Detailed Lithologic Log for Well ER-5-4

Logged by Heather Huckins-Gang, Bechtel Nevada

December, 2001

\begin{tabular}{|c|c|c|c|c|c|}
\hline $\begin{array}{c}\text { Depth } \\
\text { Interval } \\
\text { meters } \\
\text { (feet) }\end{array}$ & $\begin{array}{l}\text { Thickness } \\
\text { meters } \\
\text { (feet) }\end{array}$ & $\begin{array}{l}\text { Sample } \\
\text { Type }{ }^{1}\end{array}$ & $\begin{array}{l}\text { Laboratory } \\
\text { Analyses }^{2}\end{array}$ & Lithologic Description ${ }^{3}$ & $\begin{array}{c}\text { Stratigraphic } \\
\text { Unit }\end{array}$ \\
\hline $\begin{array}{l}0-310.9 \\
(0-1,020)\end{array}$ & $\begin{array}{c}310.9 \\
(1,020)\end{array}$ & DB1, SC & $\begin{array}{c}\text { PTS, SEM, } \\
\text { MP, XRD, } \\
\text { XRF, } \mathrm{Fe}^{2+} / \mathrm{Fe}^{3+}\end{array}$ & $\begin{array}{l}\text { Gravelly and Silty Sand: Poorly sorted, poorly indurated gravelly sand } \\
\text { or sand. Sand is tuffaceous, moderate-yellowish-brown (10YR5/4), } \\
\text { moderately to highly calcareous, very coarse to very fine and silty. } \\
\text { Thin, discontinuous carbonate coats are typically seen on up to } 50 \\
\text { percent of clasts in cuttings. Most coated (i.e. unbroken) clasts are } \\
\text { subangular to subrounded fine pebbles. Gravel clasts are almost } \\
\text { entirely volcanic but contain rare Paleozoic argillite, probably Eleana } \\
\text { Formation transported from the northeast, and very rare Paleozoic } \\
\text { carbonate and quartzite. Lithology of volcanic clasts in cuttings } \\
\text { gradually changes with depth from predominantly lighter colored ash- } \\
\text { flow tuffs of the Timber Mountain Group, transported from the north or } \\
\text { northeast, to predominantly darker lava of the Wahmonie Formation } \\
\text { transported from the east. A moderate brown (5YR4/4) slightly silty } \\
\text { and/or clayey, highly calcareous sand seen in a sidewall core at } \\
210.3 \mathrm{~m} \text { (690 ft) possibly indicates a buried soil. }\end{array}$ & \multirow[t]{2}{*}{$\begin{array}{l}\text { Quaternary or } \\
\text { Tertiary } \\
\text { alluvium }\end{array}$} \\
\hline $\begin{array}{r}310.9-704.7 \\
(1020-2312)\end{array}$ & $\begin{array}{c}393.8 \\
(1,292)\end{array}$ & DB1, SC & $\begin{array}{c}\text { PTS, SEM, } \\
\text { MP, XRD, } \\
\text { XRF, } \mathrm{Fe}^{2+} / \mathrm{Fe}^{3+}\end{array}$ & $\begin{array}{l}\text { Sand and Gravelly Sand: Poorly indurated, moderately sorted sand } \\
\text { with little silt or clay. Lesser interbeds of poorly sorted silty sand. } \\
\text { Moderate-yellowish-brown (10YR } 5 / 4) \text {; moderate-yellowish-brown } \\
(10 Y R 5 / 4) \text { to dark-yellowish-brown (10YR4/2) below approximately } \\
324.6 \mathrm{~m}(1,065 \mathrm{ft}) \text {; noncalcareous to highly calcareous. Gravel clasts in } \\
\text { cuttings are predominantly dark lava of the Wahmonie Formation with } \\
\text { very rare Paleozoic quartzite. Clayey, calcareous sand in sidewall core } \\
\text { at } 425.2 \mathrm{~m}(1,395 \mathrm{ft}) \text {. A density increase is seen on logs at } 348.7 \mathrm{~m} \\
(1,144 \mathrm{ft}) \text {. Below } 512.1 \mathrm{~m}(1,680 \mathrm{ft}) \text {, sandy matrix of alluvium is seen in } \\
\text { cuttings as fragments and coatings on clasts. }\end{array}$ & \\
\hline
\end{tabular}




\begin{tabular}{|c|c|c|c|c|c|}
\hline $\begin{array}{c}\text { Depth } \\
\text { Interval } \\
\text { meters } \\
\text { (feet) } \\
\end{array}$ & $\begin{array}{l}\text { Thickness } \\
\text { meters } \\
\text { (feet) }\end{array}$ & $\begin{array}{l}\text { Sample } \\
\text { Type }^{1}\end{array}$ & $\begin{array}{l}\text { Laboratory } \\
\text { Analyses }^{2}\end{array}$ & Lithologic Description $^{3}$ & $\begin{array}{c}\text { Stratigraphic } \\
\text { Unit }\end{array}$ \\
\hline $\begin{array}{c}704.7-774.2 \\
(2,312-2,540)\end{array}$ & $\begin{array}{l}69.5 \\
(228)\end{array}$ & DB2, SC & $\begin{array}{l}\text { PTS, SEM, } \\
\text { XRD, XRF, } \\
\mathrm{Fe}^{2+} / \mathrm{Fe}^{3+}\end{array}$ & $\begin{array}{l}\text { Silt and Clay: Moderately indurated, highly calcareous, bedded silt, } \\
\text { clay, and clayey silt; some beds sandy; biotite flakes vary from rare to } \\
\text { common. Moderate yellowish brown (10YR5/4) with lesser beds of } \\
\text { grayish-orange-pink (5YR7/2), grayish-orange (10YR7/4), and dark- } \\
\text { yellowish-orange (10YR6/6). }\end{array}$ & $\begin{array}{c}\text { Quaternary or } \\
\text { Tertiary } \\
\text { alluvium }\end{array}$ \\
\hline $\begin{array}{c}774.2-896.1 \\
(2,540-2,940)\end{array}$ & $\begin{array}{l}121.9 \\
(400)\end{array}$ & DB1, SC & $\begin{array}{c}\text { PTS, SEM, } \\
\text { MP, XRD, } \\
\text { XRF, } \mathrm{Fe}^{2+} / \mathrm{Fe}^{3+}\end{array}$ & $\begin{array}{l}\text { Silt and Clay with interbedded Sand: Silt and clay are moderately } \\
\text { indurated, highly calcareous, bedded clay, silt and clayey silt; some } \\
\text { beds are sandy. Grayish-orange (10YR7/4) to moderate-yellowish-brown } \\
\text { (10YR5/4). Sandy beds are typically moderately sorted with little silt or } \\
\text { clay, poorly indurated, calcareous, dark-yellowish-brown (10YR4/2), } \\
\text { occasionally containing concentrations of magnetite or other heavy } \\
\text { minerals. Most clasts are volcanic lithologies of the Wahmonie } \\
\text { Formation. Cuttings contain common argillite clasts, and few rounded } \\
\text { white quartzite clasts. } \\
\text { Between } 823.6 \text { and } 825.1 \mathrm{~m} \text { ( } 2,702 \text { and } 2,707 \mathrm{ft}) \text {, interval contains a } \\
\text { pale-brown (5YR5/2), highly calcareous, fine-grained ash with rare sand- } \\
\text { sized vitric pumice. Abundant black biotite and felsic phenocrysts } \\
\text { ranging from rare to common in poorly defined beds. This ash has been } \\
\text { tentatively identified as an ash-fall of the } 9.4 \text { Ma Pahute Mesa Tuff } 4 \text {. } \\
\text { A bed of dusky-yellowish-brown (10YR2/2) silty sand was noted in } \\
\text { sidewall core at } 776.0 \mathrm{~m}(2,546 \mathrm{ft}) \text {. A pumice-rich bed of silty and/or } \\
\text { clayey fine sand, probably a marginal playa deposit, was noted in } \\
\text { sidewall core at } 831.5 \mathrm{~m}(2,728 \mathrm{ft}) \text {. }\end{array}$ & $\begin{array}{l}\text { Quaternary or } \\
\text { Tertiary } \\
\text { alluvium }\end{array}$ \\
\hline
\end{tabular}




\begin{tabular}{|c|c|c|c|c|c|}
\hline $\begin{array}{l}\text { Depth } \\
\text { Interval } \\
\text { meters } \\
\text { (feet) } \\
\end{array}$ & $\begin{array}{l}\text { Thickness } \\
\text { meters } \\
\text { (feet) }\end{array}$ & $\begin{array}{l}\text { Sample } \\
\text { Type }^{1}\end{array}$ & $\begin{array}{l}\text { Laboratory } \\
\text { Analyses }^{2}\end{array}$ & Lithologic Description $^{3}$ & $\begin{array}{c}\text { Stratigraphic } \\
\text { Unit }\end{array}$ \\
\hline $\begin{array}{l}896.1-1,118.6 \\
(2,940-3,670)\end{array}$ & $\begin{array}{l}222.5 \\
(730)\end{array}$ & DB1, SC & $\begin{array}{c}\text { PTS, SEM, } \\
\text { MP, XRD, } \\
\text { XRF, } \mathrm{Fe}^{2+} / \mathrm{Fe}^{3+}\end{array}$ & $\begin{array}{l}\text { Sand and lesser Gravelly Sand: Poorly to moderately indurated, } \\
\text { calcareous to highly calcareous, typically dark-yellowish-brown } \\
(10 Y R 4 / 2) \text {. Beds with little silt or clay alternate with silty beds and beds } \\
\text { with possibly zeolitic matrix. Below } 920.5 \mathrm{~m}(3,020 \mathrm{ft}) \text { Wahmonie } \\
\text { Formation clasts in cuttings start to decrease and clasts of Timber } \\
\text { Mountain Tuff start to increase. Logs show a density increase at } \\
989.4 \mathrm{~m}(3,246 \mathrm{ft}), \text { possibly indicating an increase in zeolitic and opaline } \\
\text { alteration of matrix. }\end{array}$ & $\begin{array}{l}\text { Quaternary or } \\
\text { Tertiary } \\
\text { alluvium }\end{array}$ \\
\hline $\begin{array}{c}1,118.6-1,137.5 \\
(3,670-3,732) \\
\text { TD }\end{array}$ & $\begin{array}{l}18.9 \\
(62)\end{array}$ & DB1 & $\begin{array}{c}\text { PTS, XRD, } \\
\text { XRF, } \mathrm{Fe}^{2+} / \mathrm{Fe}^{3+}\end{array}$ & $\begin{array}{l}\text { Nonwelded to Partially Welded Ash-Flow Tuff: Pale-brown (5YR 5/2); } \\
\text { vitric to devitrified; common white (N9) pumice; minor to common felsic } \\
\text { phenocrysts of quartz and feldspar including adularescent sanidine; } \\
\text { minor biotite, trace of sphene; minor lithic fragments. }\end{array}$ & $\begin{array}{l}\text { Ammonia } \\
\text { Tanks Tuff }\end{array}$ \\
\hline
\end{tabular}

$1 \quad$ DB1 = drill cuttings enriched in hard components; $\mathbf{D B 2}=$ cuttings from interval different than that drilled; $\mathbf{S C}=$ sidewall core.

2 PTS = polished thin section; $\mathbf{S E M}=$ scanning electron microscopy; $\mathbf{M P}=$ electron microprobe; $\mathbf{X R D}=\mathbf{x}$-ray diffraction; $\mathbf{X R F}=\mathbf{x}$-ray fluorescence; $\mathrm{Fe}^{2+} / \mathrm{Fe}^{3+}=$ wet chemical analysis for iron. See Table 2-3 of this report for additional information.

3 Descriptions are based mainly on visual examination of lithologic samples using a 10x- to 40x-zoom binocular microscope, and incorporating observations from geophysical logs. Colors describe wet sample color.

Abundances for felsic phenocrysts, pumice fragments, and lithic fragments: trace = only one or two individuals observed; rare $=\leq 1 \%$; minor $=5 \%$; common $=10 \%$; abundant $=15 \%$; very abundant $=\geq 20 \%$.

Abundances for mafic minerals: trace $=$ only one or two individuals observed; rare $=\leq 0.05 \%$; minor $=0.2 \%$; common $=0.5 \%$; abundant $=1 \%$; very abundant $=\geq 2 \%$.

4 Warren, R. G., F. C. Benedict Jr., T. P. Rose, D. K. Smith, S. J. Chipera, E. C. Kluk, and K. M. Raven, 2002. "Alluvial Layering and Distribution of Reactive Phases within Drill Holes ER5/4 and UE5n of Frenchman Flat. Los Alamos National Laboratory Report LA-UR-02-6206. Los Alamos, NM. 
This page intentionally left blank.

C-1-4 
Appendix C-2

Detailed Lithologic Log for Well ER-5-4\#2 
Detailed Lithologic Log for Well ER-5-4\#2

Logged by Lance Prothro, Bechtel Nevada

October, 2002

\begin{tabular}{|c|c|c|c|c|c|}
\hline $\begin{array}{c}\text { Depth } \\
\text { Interval } \\
\text { meters (feet) } \\
\end{array}$ & $\begin{array}{c}\text { Thickness } \\
\text { meters } \\
\text { (feet) }\end{array}$ & $\begin{array}{l}\text { Sample } \\
\text { Type } 1\end{array}$ & $\begin{array}{l}\text { Laboratory } \\
\text { Analyses }\end{array}$ & Lithologic Description ${ }^{3}$ & $\begin{array}{l}\text { Stratigraphic } \\
\text { Unit }\end{array}$ \\
\hline $\begin{array}{l}0-1,120.4 \\
(0-3,676)\end{array}$ & $\begin{array}{l}1,120.4 \\
(3,676)\end{array}$ & DB1 & PTS, MP & $\begin{array}{l}\text { Alluvium: Not described. See detailed descriptions from } \\
\text { Well ER-5-4 in Appendix C-1. }\end{array}$ & $\begin{array}{c}\text { Quaternary or } \\
\text { Tertiary } \\
\text { alluvium }\end{array}$ \\
\hline $\begin{array}{l}1,120.4-1,136.9 \\
(3,676-3,730)\end{array}$ & $\begin{array}{l}16.5 \\
(54)\end{array}$ & DA & None & $\begin{array}{l}\text { Partially Welded Ash-Flow Tuff: Pale-brown ( } 5 \text { YR 5/2); devitrified, } \\
\text { partially vitric in upper part; common white (N9) and lesser } \\
\text { moderate-brown ( } 5 \text { YR } 3 / 4 \text { ) pumice; common felsic phenocrysts of } \\
\text { feldspar and slightly less quartz, some feldspar phenocrysts exhibit } \\
\text { labradorescence; minor mafic minerals of biotite and trace of } \\
\text { clinopyroxene; trace of sphene; minor volcanic lithic fragments. }\end{array}$ & \multirow{4}{*}{$\begin{array}{l}\text { Ammonia } \\
\text { Tanks Tuff }\end{array}$} \\
\hline $\begin{array}{l}1,136.9-1,167.4 \\
(3,730-3,830)\end{array}$ & $\begin{array}{l}30.5 \\
(100)\end{array}$ & DA & $\begin{array}{c}\text { PTS, XRD, } \\
\text { XRF, } \\
\mathrm{Fe}^{2+} / \mathrm{Fe}^{3+}\end{array}$ & $\begin{array}{l}\text { Moderately Welded Ash-Flow Tuff: Grayish-brown (5YR 3/2) in } \\
\text { upper part, becoming pale-brown (5YR } 5 / 2 \text { ) lower; devitrified; } \\
\text { common pale-brown ( } 5 \text { YR } 5 / 2 \text { ) pumice; common to abundant felsic } \\
\text { phenocrysts of feldspar and quartz; minor mafic minerals of biotite } \\
\text { and lesser clinopyroxene; trace of sphene; rare volcanic lithic } \\
\text { fragments. }\end{array}$ & \\
\hline $\begin{array}{l}1,167.4-1,185.7 \\
(3,830-3,890)\end{array}$ & $\begin{array}{l}18.3 \\
(60)\end{array}$ & DA & $\begin{array}{l}\text { PTS, MP, } \\
\text { XRD, XRF, } \\
\mathrm{Fe}^{2+} / \mathrm{Fe}^{3+}\end{array}$ & $\begin{array}{l}\text { Partially Welded Ash-Flow Tuff: Yellowish-gray (5Y 8/1) and light- } \\
\text { brownish-gray (5YR 6/1); devitrified; rare medium-light-gray (N6) } \\
\text { pumice; minor felsic phenocrysts of quartz and feldspar, some } \\
\text { feldspar phenocrysts exhibit labradorescence; minor mafic minerals } \\
\text { of biotite and much less clinopyroxene; trace of sphene; rare } \\
\text { volcanic lithic fragments. }\end{array}$ & \\
\hline $\begin{array}{c}1,185.7-1,197.3 \\
(3,890-3,928)\end{array}$ & $\begin{array}{l}11.6 \\
(38)\end{array}$ & DA & None & $\begin{array}{l}\text { Nonwelded Ash-Flow Tuff: Dark-yellowish-orange (10YR 6/6); } \\
\text { vitric; minor very-pale-orange (10YR 8/2) pumice; minor to common } \\
\text { felsic phenocrysts of feldspar and quartz; rare to minor biotite; minor } \\
\text { volcanic lithic fragments; very abundant and conspicuous orange } \\
\text { cuspate glass shards. }\end{array}$ & \\
\hline
\end{tabular}




\begin{tabular}{|c|c|c|c|c|c|}
\hline $\begin{array}{c}\text { Depth } \\
\text { Interval } \\
\text { meters (feet) }\end{array}$ & $\begin{array}{c}\text { Thickness } \\
\text { meters } \\
\text { (feet) }\end{array}$ & $\begin{array}{l}\text { Sample } \\
\text { Type } 1\end{array}$ & $\begin{array}{l}\text { Laboratory } \\
\text { Analyses }\end{array}$ & Lithologic Description ${ }^{3}$ & $\begin{array}{c}\text { Stratigraphic } \\
\text { Unit }\end{array}$ \\
\hline $\begin{array}{c}1,197.3-1,213.1 \\
(3,928-3,980)\end{array}$ & $\begin{array}{l}15.8 \\
(52)\end{array}$ & DB1 & $\begin{array}{c}\text { PTS, XRD, } \\
\text { XRF, } \\
\mathrm{Fe}^{2+} / \mathrm{Fe}^{3+}\end{array}$ & $\begin{array}{l}\text { Bedded Tuff: Drill cuttings samples consist mainly of a varied } \\
\text { mixture of lava and welded tuff fragments from various, and } \\
\text { probably older, stratigraphic units. Many fragments have thin } \\
\text { coatings of fine-sand-size, vitric, tuffaceous material that is weakly } \\
\text { calcareous and has a composition similar to that of Ammonia Tanks } \\
\text { Tuff. Most of the fragments likely represent hard lithic fragments } \\
\text { liberated from poorly consolidated bedded tuffs during the drilling } \\
\text { process. }\end{array}$ & $\begin{array}{l}\text { bedded, } \\
\text { Ammonia } \\
\text { Tanks Tuff }\end{array}$ \\
\hline $\begin{array}{c}1,213.1-1,225.3 \\
(3,980-4,020)\end{array}$ & $\begin{array}{l}12.2 \\
(40)\end{array}$ & DA & $\begin{array}{c}\text { PTS, XRD, } \\
\text { XRF, } \\
\mathrm{Fe}^{2+} / \mathrm{Fe}^{3+}\end{array}$ & $\begin{array}{l}\text { Nonwelded Ash-Flow Tuff: Pale-reddish-brown ( } 10 \mathrm{R} 5 / 4) \text {; mostly } \\
\text { vitric, partially devitrified; common white (N9) pumice; minor to } \\
\text { common felsic phenocrysts of feldspar and quartz; common biotite; } \\
\text { rare volcanic lithic fragments. }\end{array}$ & \multirow{5}{*}{$\begin{array}{c}\text { Rainier Mesa } \\
\text { Tuff }\end{array}$} \\
\hline $\begin{array}{c}1,225.3-1,250.3 \\
(4,020-4,102)\end{array}$ & $\begin{array}{l}25.0 \\
(82)\end{array}$ & DA & None & $\begin{array}{l}\text { Partially Welded Ash-Flow Tuff: Light-brownish-gray ( } 5 \text { YR } 6 / 1) \text {; } \\
\text { devitrified; common brownish-gray (5YR 4/1) pumice; common felsic } \\
\text { phenocrysts of feldspar and quartz; minor to common biotite; rare } \\
\text { volcanic lithic fragments. }\end{array}$ & \\
\hline $\begin{array}{c}1,250.3-1,312.5 \\
(4,102-4,306)\end{array}$ & $\begin{array}{l}62.2 \\
(204)\end{array}$ & DA & $\begin{array}{c}\text { PTS, XRD, } \\
\text { XRF, } \\
\mathrm{Fe}^{2+} / \mathrm{Fe}^{3+}\end{array}$ & $\begin{array}{l}\text { Moderately Welded Ash-Flow Tuff: Medium-gray (N5) to } \\
\text { brownish-gray (5YR 4/1); devitrified; minor brownish-gray ( } 5 \text { YR 4/1) } \\
\text { pumice; minor felsic phenocrysts of feldspar and lesser quartz; } \\
\text { minor to common biotite; rare volcanic lithic fragments. } \\
\text { Geophysical logs indicate degree of welding increases with depth. }\end{array}$ & \\
\hline $\begin{array}{c}1,312.5-1,316.7 \\
(4,306-4,320)\end{array}$ & $\begin{array}{c}4.3 \\
(14)\end{array}$ & DA & None & Partially Welded Ash-Flow Tuff: Indicated by geophysical logs. & \\
\hline $\begin{array}{c}1,316.7-1,327.7 \\
(4,320-4,356)\end{array}$ & $\begin{array}{l}11.0 \\
(36)\end{array}$ & DA & None & $\begin{array}{l}\text { Nonwelded Ash-Flow Tuff: Moderate-brown ( } 5 \text { YR } 4 / 4) \text {; vitric; } \\
\text { common grayish-orange-pink ( } 5 \text { YR } 7 / 2 \text { ) pumice; common felsic } \\
\text { phenocrysts of feldspar and quartz; rare biotite; rare volcanic lithic } \\
\text { fragments; common cuspate glass shards. }\end{array}$ & \\
\hline
\end{tabular}




\begin{tabular}{|c|c|c|c|c|c|}
\hline $\begin{array}{c}\text { Depth } \\
\text { Interval } \\
\text { meters (feet) }\end{array}$ & $\begin{array}{c}\text { Thickness } \\
\text { meters } \\
\text { (feet) }\end{array}$ & $\begin{array}{l}\text { Sample } \\
\text { Type }^{1}\end{array}$ & $\begin{array}{l}\text { Laboratory } \\
\text { Analyses }^{2}\end{array}$ & Lithologic Description ${ }^{3}$ & $\begin{array}{c}\text { Stratigraphic } \\
\text { Unit }\end{array}$ \\
\hline $\begin{array}{c}1,327.7-1,363.1 \\
(4,356-4,472)\end{array}$ & $\begin{array}{l}35.4 \\
(116)\end{array}$ & $\begin{array}{l}\text { DB2, } \\
\text { DB4 }\end{array}$ & $\begin{array}{c}\text { PTS, XRD, } \\
\text { XRF, } \\
\mathrm{Fe}^{2+} / \mathrm{Fe}^{3+}\end{array}$ & $\begin{array}{l}\text { Nonwelded and Bedded Tuff: A detailed lithologic description is } \\
\text { not possible due to the poor quality of drill cuttings samples, which } \\
\text { consist of highly varied mixtures of volcanic rock fragments } \\
\text { generally less than } 3 \mathrm{~mm} \text { in size. Approximately } 25 \% \text { or more of } \\
\text { the fragments in samples are moderately welded, devitrified, Rainier } \\
\text { Mesa Tuff from the interval } 1,250.3-1,312.5 \mathrm{~m}(4,102-4,306 \mathrm{ft}) \text {, } \\
\text { indicating considerable contamination of the samples from higher } \\
\text { lithologic intervals. Lithologic and stratigraphic determinations are } \\
\text { based on information from regional geology, lithologic and } \\
\text { stratigraphic assignments of over- and underlying intervals, and } \\
\text { geophysical logs. }\end{array}$ & $\begin{array}{c}\text { pre-Rainier } \\
\text { Mesa Tuff/ } \\
\text { Post- } \\
\text { Wahmonie } \\
\text { Formation }\end{array}$ \\
\hline
\end{tabular}




\begin{tabular}{|c|c|c|c|c|c|}
\hline $\begin{array}{c}\text { Depth } \\
\text { Interval } \\
\text { meters (feet) }\end{array}$ & $\begin{array}{c}\text { Thickness } \\
\text { meters } \\
\text { (feet) }\end{array}$ & $\begin{array}{l}\text { Sample } \\
\text { Type } 1\end{array}$ & $\begin{array}{l}\text { Laboratory } \\
\text { Analyses }\end{array}$ & Lithologic Description ${ }^{3}$ & $\begin{array}{c}\text { Stratigraphic } \\
\text { Unit }\end{array}$ \\
\hline $\begin{array}{c}1,363.1-1,553.3 \\
(4,472-5,096)\end{array}$ & $\begin{array}{l}190.2 \\
(624)\end{array}$ & $\begin{array}{l}\text { DB2, } \\
\text { DB4, } \\
\text { SC }\end{array}$ & $\begin{array}{c}\text { PTS, XRD, } \\
\text { XRF, } \\
\mathrm{Fe}^{2+} / \mathrm{Fe}^{3+}\end{array}$ & $\begin{array}{l}\text { Nonwelded Tuff: Olive-gray ( } 5 \mathrm{Y} 4 / 1) \text { and dusky-yellow-green ( } 5 \mathrm{GY} \\
5 / 2) \text {; zeolitic; rare to minor pumice; common to abundant feldspar } \\
\text { phenocrysts; very abundant mafic minerals of orthopyroxene and } \\
\text { lesser biotite; common to very abundant volcanic lithic fragments. } \\
\text { This description is based on small (< } 2 \mathrm{~cm} \text { in diameter) fragments of } \\
\text { sidewall core samples obtained from the } 1,366.4 \mathrm{~m}(4,483 \mathrm{ft}) \text { and } \\
1,414.0 \mathrm{~m}(4,639 \mathrm{ft}) \text { depths, and thus appropriate uncertainties } \\
\text { should be considered when applying the description to the entire } \\
\text { interval. Drill cuttings samples throughout interval are of very poor } \\
\text { quality and are not representative of the rocks of the interval. Drill } \\
\text { cuttings samples appear to consist mainly of material caved from } \\
\text { higher intervals. This is indicated by the observation that } \\
\text { approximately } 25 \% \text { or more of the fragments in samples above } \\
1,432.6 \mathrm{~m}(4,700 \mathrm{ft}) \text { are moderately welded, devitrified, Rainier Mesa } \\
\text { Tuff from the interval } 1,250.3-1,312.5 \mathrm{~m} \text { ( } 4,102-4,306 \mathrm{ft}) \text {. The } \\
\text { zeolitic, nonwelded, nature of the interval is indicated by the } \\
\text { relatively low resistivity of the interval as measured by the dual } \\
\text { laterolog. The upper contact corresponds to an abrupt decrease in } \\
\text { natural gamma radiation as observed on gamma ray logs. The } \\
\text { lower contact is near the bottom of the } 121 / 4-\text { in. hole and is based } \\
\text { partly on the general depth where drill cuttings samples change from } \\
\text { being highly mixed and severely contaminated by higher, pre- } \\
\text { Wahmonie units to highly mixed samples that are dominated by } \\
\text { mafic-rich fragments representative of the upper portions of the } \\
\text { Wahmonie Formation. This change is due to casing being set at } \\
1,477.9 \mathrm{~m}(4,848.8 \mathrm{ft}) \text {, which eliminates post-Wahmonie sources of } \\
\text { contamination. }\end{array}$ & $\begin{array}{l}\text { Wahmonie } \\
\text { Formation }\end{array}$ \\
\hline
\end{tabular}




\begin{tabular}{|c|c|c|c|c|c|}
\hline $\begin{array}{c}\text { Depth } \\
\text { Interval } \\
\text { meters (feet) }\end{array}$ & $\begin{array}{c}\text { Thickness } \\
\text { meters } \\
\text { (feet) }\end{array}$ & $\begin{array}{l}\text { Sample } \\
\text { Type }\end{array}$ & $\begin{array}{l}\text { Laboratory } \\
\text { Analyses }^{2}\end{array}$ & Lithologic Description ${ }^{3}$ & $\begin{array}{c}\text { Stratigraphic } \\
\text { Unit }\end{array}$ \\
\hline $\begin{array}{c}1,553.3-1,850.7 \\
(5,096-6,072)\end{array}$ & $\begin{array}{l}297.5 \\
(976)\end{array}$ & DB4 & $\begin{array}{c}\mathrm{PTS}, \mathrm{MP} \\
\mathrm{XRD}, \mathrm{XRF} \\
\mathrm{Fe}^{2+} / \mathrm{Fe}^{3+}\end{array}$ & $\begin{array}{l}\text { Nonwelded and Bedded Tuff: Greenish-gray ( } 5 G \text { G/1), light- } \\
\text { greenish-gray (5G 8/1), light-brownish-gray ( } 5 \text { YR } 6 / 1) \text {, and light- } \\
\text { brown ( } 5 \text { YR } 5 / 6) \text {; zeolitic; rare to minor pumice; common to } \\
\text { abundant feldspar phenocrysts; abundant to very abundant mafic } \\
\text { minerals of biotite and lesser orthopyroxene; minor to abundant } \\
\text { volcanic lithic fragments consisting mostly of very dense welded } \\
\text { tuff and lava. } \\
\text { Drill cuttings samples are of poor quality. They consist of a varied } \\
\text { mixture of various volcanic rock fragments generally less than } \\
3 \text { mm in size, and are likely mixed and contaminated with material } \\
\text { from various horizons. The representative lithology in most } \\
\text { samples from the interval appears to be poorly welded, mafic-rich, } \\
\text { zeolitic tuff as described above. The relatively low resistivity } \\
\text { throughout the interval as measured on the dual laterolog is } \\
\text { consistent with poorly-welded, zeolitic tuff. Several intervals of } \\
\text { samples mostly contain fragments of moderately- to densely-welded } \\
\text { tuff and dense lava, and may represent more lithic-rich intervals. } \\
\text { Slightly higher density values from geophysical logs correspond to } \\
\text { these sample depths. }\end{array}$ & \multirow[t]{2}{*}{$\begin{array}{l}\text { Wahmonie } \\
\text { Formation }\end{array}$} \\
\hline $\begin{array}{c}1,850.7-1,869.6 \\
(6,072-6,134)\end{array}$ & $\begin{array}{l}18.9 \\
(62)\end{array}$ & DB4 & None & $\begin{array}{l}\text { Lava: Grayish-brown ( } 5 \text { YR } 3 / 2) \text {, with a mottled appearance; } \\
\text { devitrified, with sucrose texture that may indicate silicic alteration; } \\
\text { common feldspar phenocrysts; rare to minor, very small, unaltered } \\
\text { to partially altered biotite; common to abundant unknown moderate- } \\
\text { green ( } 5 \mathrm{G} 5 / 6) \text { pseudomorphs after biotite(?). No pumice or lithic } \\
\text { fragments observed. } \\
\text { Drill cuttings samples are of poor quality. They consist of a varied } \\
\text { mixture of volcanic rock fragments generally less than } 3 \mathrm{~mm} \text { in } \\
\text { size, and are likely mixed and contaminated with material from } \\
\text { various horizons. Recognition of interval is based mainly on a } \\
\text { conspicuous, abrupt increase in density and resistivity as observed } \\
\text { on geophysical logs. The abrupt contacts are more indicative of a } \\
\text { lava than a welded ash-flow tuff. }\end{array}$ & \\
\hline
\end{tabular}




\begin{tabular}{|c|c|c|c|c|c|}
\hline $\begin{array}{c}\text { Depth } \\
\text { Interval } \\
\text { meters (feet) }\end{array}$ & $\begin{array}{c}\text { Thickness } \\
\text { meters } \\
\text { (feet) }\end{array}$ & $\begin{array}{l}\text { Sample } \\
\text { Type } 1\end{array}$ & $\begin{array}{l}\text { Laboratory } \\
\text { Analyses }\end{array}$ & Lithologic Description ${ }^{3}$ & $\begin{array}{c}\text { Stratigraphic } \\
\text { Unit }\end{array}$ \\
\hline $\begin{array}{c}1,869.6-1,903.8 \\
(6,134-6,246)\end{array}$ & $\begin{array}{l}34.1 \\
(112)\end{array}$ & DB4 & $\begin{array}{c}\text { PTS, MP, } \\
\text { XRD, XRF, } \\
\mathrm{Fe}^{2+} / \mathrm{Fe}^{3+}\end{array}$ & $\begin{array}{l}\text { Nonwelded Tuff: Light-brownish-gray (5YR 6/1); zeolitic; common } \\
\text { white (N9) pumice; common to abundant feldspar phenocrysts; very } \\
\text { abundant mafic minerals of mostly biotite and lesser partially altered } \\
\text { orthopyroxene(?); minor to common volcanic lithic fragments. } \\
\text { Drill cuttings samples are of poor quality, They consist of a varied } \\
\text { mixture of volcanic rock fragments generally less than } 3 \mathrm{~mm} \text { in } \\
\text { size, and are likely mixed and contaminated with material from } \\
\text { various horizons. }\end{array}$ & $\begin{array}{l}\text { Wahmonie } \\
\text { Formation }\end{array}$ \\
\hline $\begin{array}{c}1,903.8-1,922.1 \\
(6,246-6,306)\end{array}$ & $\begin{array}{l}18.3 \\
(60)\end{array}$ & DA & None & $\begin{array}{l}\text { Nonwelded Tuff: Moderate-yellow ( } 5 \mathrm{Y} 7 / 6) \text { at top of interval, } \\
\text { becoming moderate-reddish-brown ( } 10 \mathrm{R} 4 / 6) \text { lower; zeolitic; common } \\
\text { pumice; minor felsic phenocrysts feldspar and quartz; rare to minor } \\
\text { biotite; rare volcanic lithic fragments. }\end{array}$ & \\
\hline $\begin{array}{c}1,922.1-2,051.3 \\
(6,306-6,730)\end{array}$ & $\begin{array}{l}129.2 \\
(424)\end{array}$ & $\begin{array}{l}\text { DA, } \\
\text { DB4 }\end{array}$ & $\begin{array}{l}\text { PTS, MP, } \\
\text { XRD, XRF, } \\
\mathrm{Fe}^{2+} / \mathrm{Fe}^{3+}\end{array}$ & $\begin{array}{l}\text { Nonwelded Tuff: Grayish-orange (10YR } 7 / 4) \text { at top of interval, } \\
\text { becoming grayish-red (10R } 4 / 2) \text { lower and moderate-yellowish-brown } \\
\text { (10YR } 5 / 4 \text { ) at base of interval; zeolitic; minor to common pumice; } \\
\text { minor felsic phenocrysts of feldspar and quartz; minor to common } \\
\text { biotite; minor volcanic lithic fragments. The spectral gamma ray log } \\
\text { indicates fractures at } 1,932.1 \mathrm{~m}(6,339 \mathrm{ft}), 1,953.8 \mathrm{~m}(6,410 \mathrm{ft}) \text {, } \\
1,970.2 \mathrm{~m}(6,464 \mathrm{ft}) \text {, and } 1,980.9 \mathrm{~m}(6,499 \mathrm{ft}) \text {. The lack of an } \\
\text { increase in water production during drilling of these depths, and } \\
\text { absence of borehole breakouts on the caliper log suggest fractures } \\
\text { are closed/healed. An interval of large open fractures from } 1,998.0 \text { - } \\
2,024.5 \mathrm{~m} \text { ( } 6,555-6,642 \mathrm{ft}) \text { is indicated by the caliper log and by an } \\
\text { increase in water production during drilling of this depth interval. } \\
\text { Missing samples at } 2,005.6-2,011.7 \mathrm{~m}(6,580-6,600 \mathrm{ft}), 2,014.7 \text { - } \\
2,020.8 \mathrm{~m}(6,610-6,630 \mathrm{ft}) \text {, and } 2,023.9-2,033.0 \mathrm{~m}(6,640- \\
6,670 \mathrm{ft}) \text { due to lost circulation during drilling at these depths is also } \\
\text { indicative of open fractures. } \\
\text { Although the drill cuttings samples degrade in quality below } \\
1,996.4 \mathrm{~m}(6,550 \mathrm{ft}) \text {, becoming varied mixtures of volcanic units, } \\
\text { geophysical logs suggest the lithology is generally consistent } \\
\text { throughout interval. }\end{array}$ & Bullfrog Tuff \\
\hline
\end{tabular}




\begin{tabular}{|c|c|c|c|c|c|}
\hline $\begin{array}{c}\text { Depth } \\
\text { Interval } \\
\text { meters (feet) }\end{array}$ & $\begin{array}{c}\text { Thickness } \\
\text { meters } \\
\text { (feet) }\end{array}$ & $\begin{array}{l}\text { Sample } \\
\text { Type } 1\end{array}$ & $\begin{array}{l}\text { Laboratory } \\
\text { Analyses }\end{array}$ & Lithologic Description ${ }^{3}$ & $\begin{array}{c}\text { Stratigraphic } \\
\text { Unit }\end{array}$ \\
\hline $\begin{array}{c}2,051.3-2,133.6 \\
(6,730-7,000)\end{array}$ & $\begin{array}{l}82.3 \\
(270)\end{array}$ & DB4 & $\begin{array}{c}\text { PTS, XRD, } \\
\text { XRF, } \\
\mathrm{Fe}^{2+} / \mathrm{Fe}^{3+}\end{array}$ & $\begin{array}{l}\text { Nonwelded Tuff: Medium-dark-gray (N4) and light-olive-gray } \\
\text { ( } 5 \text { Y } 6 / 1) \text {; zeolitic; minor pumice; common to very abundant feldspar } \\
\text { phenocrysts; very abundant biotite; common to abundant lithic } \\
\text { fragments. Fragments of grayish-brown ( } 5 \text { YR } 3 / 2 \text { ) tuffaceous } \\
\text { siltstone compose less than } 25 \% \text { by volume in samples below } \\
\text { approximately } 2,072.6 \mathrm{~m} \text { ( } 6,800 \mathrm{ft}) \text {. } \\
\text { Drill cuttings samples throughout interval are of poor quality. They } \\
\text { consist of a varied mixture of volcanic rock units, and may not be } \\
\text { representative of the rocks comprising the interval. However, the } \\
\text { dominance of tuffaceous fragments, particularly fragments of poorly } \\
\text { welded tuff, and the low resistivity of the interval suggest the } \\
\text { interval is composed of poorly welded, zeolitic tuff. The biotite-rich, } \\
\text { quartz-poor character of most fragments is characteristic of the } \\
\text { Wahmonie Formation and may indicate that the lower portion of the } \\
\text { Wahmonie Formation is older than the Bullfrog Tuff. }\end{array}$ & $\begin{array}{l}\text { Wahmonie } \\
\text { Formation }\end{array}$ \\
\hline
\end{tabular}

$1 \quad \mathbf{D A}=$ drill cuttings that represent lithologic character of interval; $\mathbf{D B} 1=$ drill cuttings enriched in hard components; $\mathbf{D B 2}=$ cuttings from interval different than that drilled; DB4 = cuttings that are intimate mixtures of units; $\mathbf{S C}=$ sidewall core.

2 PTS = polished thin section; $\mathbf{M P}=$ electron microprobe $\mathbf{X R D}=\mathrm{x}$-ray diffraction; $\mathbf{X R F}=\mathrm{x}$-ray fluorescence; $\mathrm{Fe}^{2+} / \mathrm{Fe}^{3+}=$ wet chemical analysis for iron. See Table 3-3 of this report for additional information.

3 Descriptions are based mainly on visual examination of lithologic samples using a 10x-to 40x-zoom binocular microscope, and incorporating observations from geophysical logs and results of laboratory analyses. Colors describe wet sample color.

Abundances for felsic phenocrysts, pumice fragments, and lithic fragments: trace = only one or two individuals observed; rare $=\leq 1 \%$; minor $=5 \%$; common $=10 \%$; abundant $=15 \%$; very abundant $\geq 20 \%$.

Abundances for mafic minerals: trace $=$ only one or two individuals observed; rare $=\leq 0.05 \%$; minor $=0.2 \%$; common $=0.5 \%$; abundant $=1 \%$; very abundant $=\geq 2 \%$. 
This page intentionally left blank.

C-2-8 


\section{Appendix D Geophysical Logs Run at Well Cluster ER5-4}

D-1 Geophysical Logs Run in Well ER-5-4

D-2 Geophysical Logs Run in Well ER-5-4\#2 


\section{Appendix D-1 \\ Geophysical Logs Run in Well ER5-4}


Appendix D-1 contains unprocessed data presentations of selected geophysical logs run in

Well ER-5-4. Table D-1-1 summarizes the logs presented for Well ER-5-4. See Table 2-4 for more information.

Table D-1-1

Well ER-5-4 Geophysical Logs Presented

\begin{tabular}{|c|c|c|c|c|}
\hline Log Type & $\begin{array}{c}\text { Run } \\
\text { Number }\end{array}$ & Date & meters $^{\text {Log }}$ & val feet \\
\hline Caliper & $\begin{array}{l}\text { CA4-1 } \\
\text { CA4-2 } \\
\text { CA4-5 }\end{array}$ & $\begin{array}{l}02 / 16 / 2001 \\
03 / 09 / 2001 \\
03 / 24 / 2001\end{array}$ & $\begin{array}{c}36.3-252.1 \\
194.5-508.7 \\
492.6-1,099.7\end{array}$ & $\begin{array}{c}119-827 \\
638-1,669 \\
1,616-3,608\end{array}$ \\
\hline $\begin{array}{l}\text { Epithermal Neutron } \\
\text { (porosity) }\end{array}$ & $\begin{array}{l}\text { ENP-1 } \\
\text { ENP-2 } \\
\text { ENP-3 }\end{array}$ & $\begin{array}{l}02 / 16 / 2001 \\
03 / 09 / 2001 \\
03 / 25 / 2001\end{array}$ & $\begin{array}{c}36.6-246.9 \\
235.3-502.3 \\
395.6-1,090.3\end{array}$ & $\begin{array}{c}119-810 \\
772-1,648 \\
1,298-3,577\end{array}$ \\
\hline Density & $\begin{array}{l}\text { CDL-1 } \\
\text { CDL-2 } \\
\text { CDL-3 }\end{array}$ & $\begin{array}{l}02 / 16 / 2001 \\
03 / 09 / 2001 \\
03 / 25 / 2001\end{array}$ & $\begin{array}{c}36.6-246.9 \\
235.3-502.3 \\
395.6-1,090.3\end{array}$ & $\begin{array}{c}119-810 \\
772-1,648 \\
1,298-3,577\end{array}$ \\
\hline $\begin{array}{l}\text { Array Induction and Dual Laterolog } \\
\text { (resistivity) }\end{array}$ & $\begin{array}{l}\text { AIT-1 } \\
\text { AIT-2 } \\
\text { DLL-1 }\end{array}$ & $\begin{array}{l}02 / 16 / 2001 \\
03 / 09 / 2001 \\
03 / 25 / 2001\end{array}$ & $\begin{array}{c}36.3-250.0 \\
235.3-505.4 \\
509.9-1,093.3\end{array}$ & $\begin{array}{c}118-820 \\
772-1,658 \\
1,673-3,587\end{array}$ \\
\hline Spontaneous Potential & $\begin{array}{l}\text { SP-1 } \\
\text { SP-2 } \\
\text { SP-3 }\end{array}$ & $\begin{array}{l}02 / 16 / 2001 \\
03 / 09 / 2001 \\
03 / 25 / 2001\end{array}$ & $\begin{array}{c}36.3-250.0 \\
235.3-505.4 \\
396.2-1,510.3\end{array}$ & $\begin{array}{c}118-820 \\
772-1,648 \\
1,300-4,955\end{array}$ \\
\hline Gamma Ray & $\begin{array}{l}\text { GR-1 } \\
\text { GR-3 } \\
\text { GR-7 }\end{array}$ & $\begin{array}{l}02 / 16 / 2001 \\
03 / 09 / 2001 \\
03 / 25 / 2001\end{array}$ & $\begin{array}{c}36.3-252.1 \\
235.3-505.4 \\
395.6-1,090.3\end{array}$ & $\begin{array}{c}119-827 \\
772-1,658 \\
1,298-3,577\end{array}$ \\
\hline $\begin{array}{c}\text { Digital Array Sonic } \\
\text { (delta T and sonic porosity) }\end{array}$ & AC-1 & $03 / 25 / 2001$ & $460.3-1,090.6$ & $1,510-3,578$ \\
\hline $\begin{array}{c}\text { Spectral Gamma Ray } \\
\text { (potassium, thorium, uranium) }\end{array}$ & $\begin{array}{l}\text { SGR-1 } \\
\text { SGR-2 } \\
\text { SGR-3 }\end{array}$ & $\begin{array}{l}02 / 17 / 2001 \\
03 / 09 / 2001 \\
03 / 25 / 2001\end{array}$ & $\begin{array}{c}36.3-243.2 \\
194.5-496.8 \\
460.2-1,090.6\end{array}$ & $\begin{array}{c}199-798 \\
638-1,630 \\
1,510-3,578\end{array}$ \\
\hline
\end{tabular}




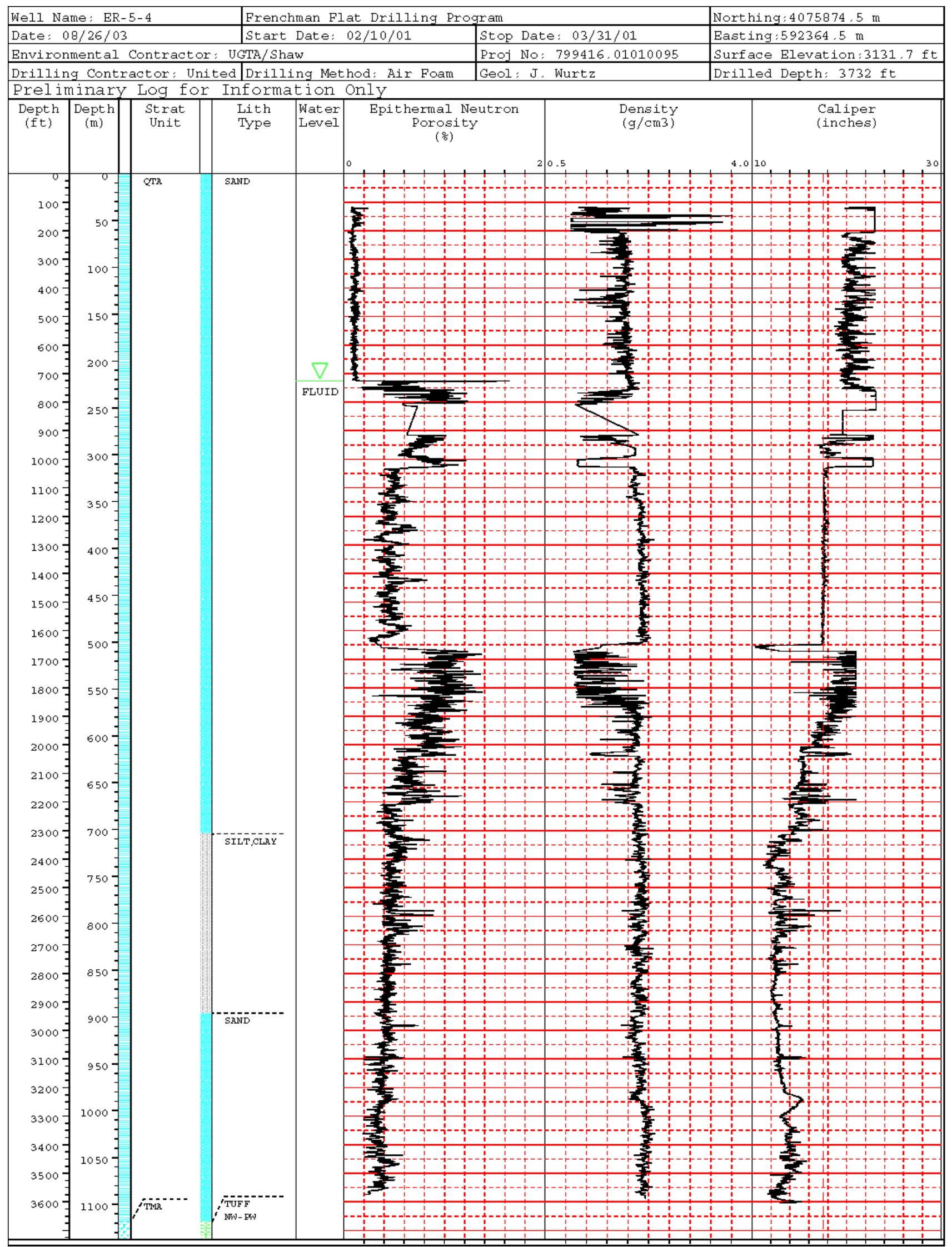

D-1-2 


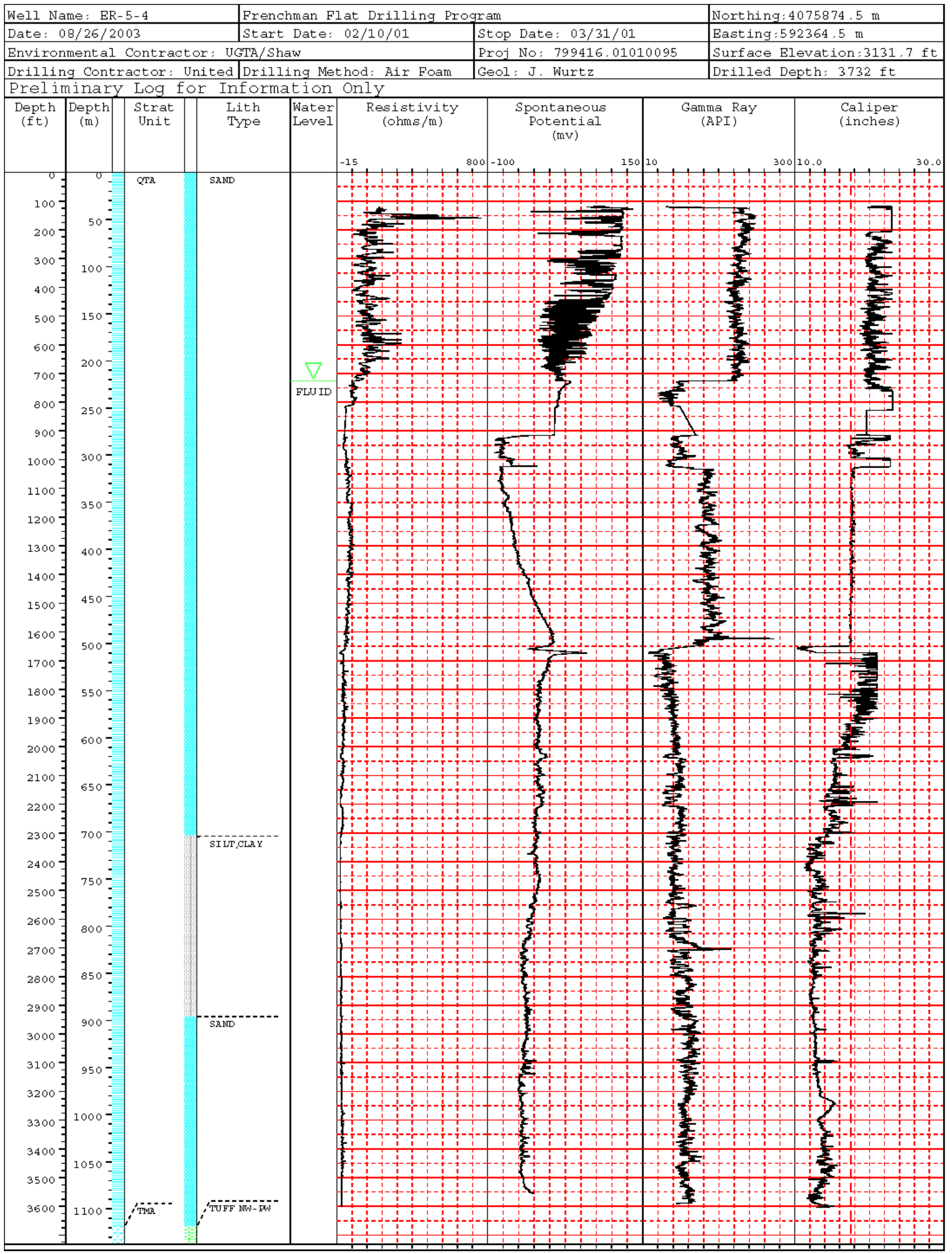

D-1-3 


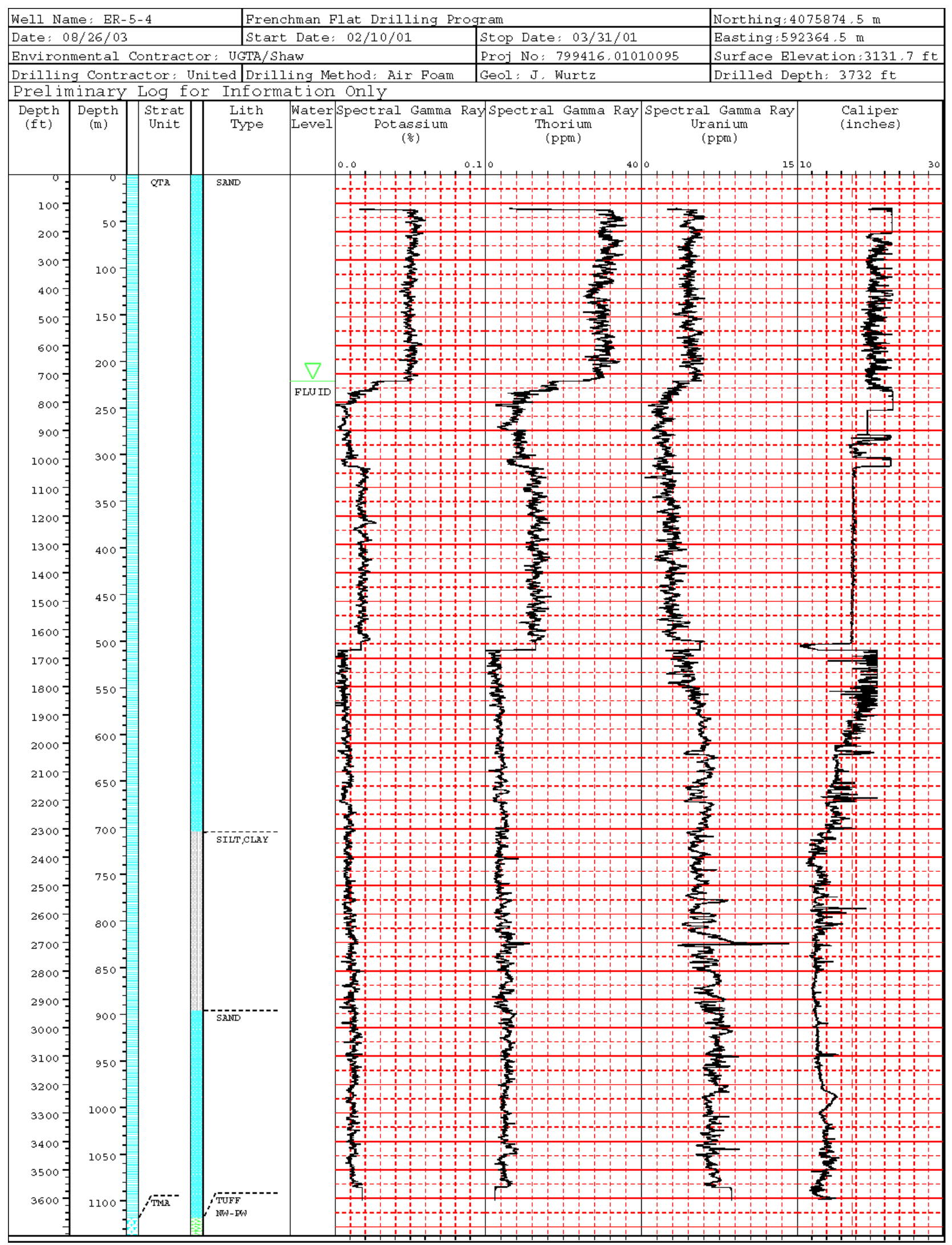

D-1-4 


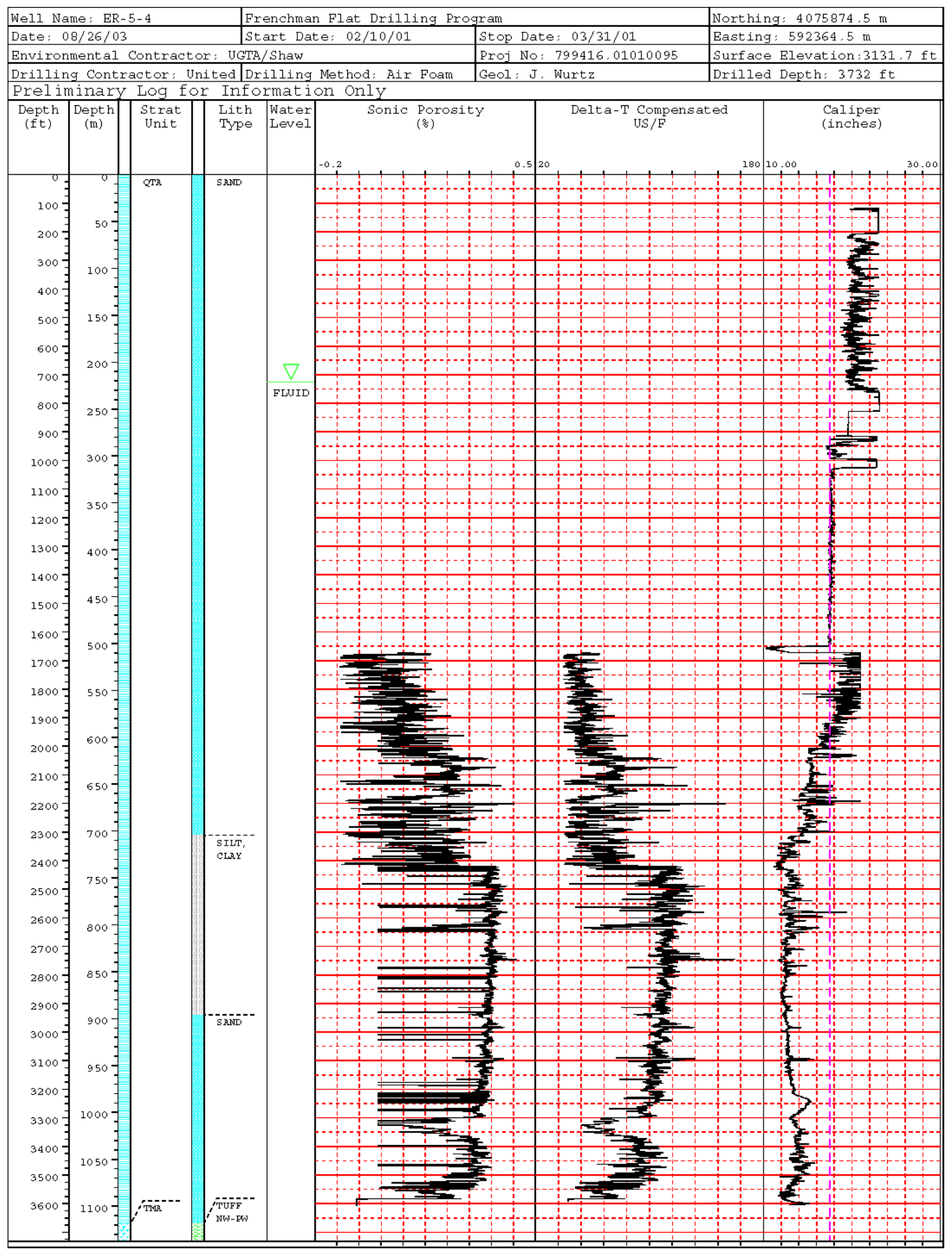


This page intentionally left blank.

D-1-6 
Appendix D-2

Geophysical Logs Run in Well ER5-4\#2 
Appendix D-2 contains unprocessed data presentations of selected geophysical logs run in Well ER-5-4\#2. Table D-2-1 summarizes the logs presented for Well ER-5-4\#2. See Table 3-4 for more information.

Table D-2-1

Well ER-5-4\#2 Geophysical Logs Presented

\begin{tabular}{|c|c|c|c|c|}
\hline Log Type & $\begin{array}{c}\text { Run } \\
\text { Number }\end{array}$ & Date & $\begin{array}{l}\text { Log In } \\
\text { meters }\end{array}$ & $\begin{array}{l}\text { val } \\
\text { feet }\end{array}$ \\
\hline Caliper & $\begin{array}{l}\text { CA6-1 } \\
\text { CA6-2 }\end{array}$ & $\begin{array}{l}08 / 28 / 2002 \\
09 / 13 / 2002\end{array}$ & $\begin{array}{c}923.5-1,477.8 \\
1,356.2-2,128.4\end{array}$ & $\begin{array}{l}3,030-4,848 \\
4,450-6,983\end{array}$ \\
\hline $\begin{array}{l}\text { Epithermal Neutron } \\
\text { (porosity) }\end{array}$ & $\begin{array}{l}\text { DSEN-1 } \\
\text { DSEN-3 }\end{array}$ & $\begin{array}{l}08 / 28 / 2002 \\
09 / 15 / 2002\end{array}$ & $\begin{array}{c}838.2-1,479.5 \\
1,447.8-2,131.8\end{array}$ & $\begin{array}{l}2,750-4,854 \\
4,750-6,994\end{array}$ \\
\hline Density & $\begin{array}{l}\text { CDL-1 } \\
\text { CDL-3 }\end{array}$ & $\begin{array}{l}08 / 28 / 2002 \\
09 / 15 / 2002\end{array}$ & $\begin{array}{c}838.2-1,479.5 \\
1,447.8-2,131.8\end{array}$ & $\begin{array}{l}2,750-4,854 \\
4,750-6,994\end{array}$ \\
\hline $\begin{array}{l}\text { Dual Laterolog } \\
\text { (resistivity) }\end{array}$ & $\begin{array}{l}\text { DLL-1 } \\
\text { DLL-2 }\end{array}$ & $\begin{array}{l}08 / 28 / 2002 \\
09 / 14 / 2002\end{array}$ & $\begin{array}{c}944.9-1,477.1 \\
1,447.8-2,126.9\end{array}$ & $\begin{array}{l}3,100-4,846 \\
4,750-6,978\end{array}$ \\
\hline Spontaneous Potential & $\begin{array}{l}\text { SP-1 } \\
\text { SP-2 }\end{array}$ & $\begin{array}{l}08 / 28 / 2002 \\
09 / 14 / 2002\end{array}$ & $\begin{array}{c}944.9-1,477.1 \\
1,447.8-2,126.9\end{array}$ & $\begin{array}{l}3,100-4,846 \\
4,750-6,978\end{array}$ \\
\hline Gamma Ray & $\begin{array}{l}\text { GR-1 } \\
\text { GR-11 }\end{array}$ & $\begin{array}{l}08 / 28 / 2002 \\
09 / 15 / 2002\end{array}$ & $\begin{array}{l}923.5-1,477.8 \\
1,447.8-2,125.4\end{array}$ & $\begin{array}{l}3,030-4,848 \\
4,750-6,973\end{array}$ \\
\hline $\begin{array}{c}\text { Digital Array Sonic } \\
\text { (delta T and sonic porosity) }\end{array}$ & $\begin{array}{l}\text { FWS-1 } \\
\text { FWS-2 }\end{array}$ & $\begin{array}{l}08 / 28 / 2002 \\
09 / 14 / 2002\end{array}$ & $\begin{array}{c}941.8-1,465.5 \\
1,447.8-2,117.8\end{array}$ & $\begin{array}{l}3,090-4,808 \\
4,750-6,948\end{array}$ \\
\hline $\begin{array}{c}\text { Spectral Gamma Ray } \\
\text { (potassium, thorium, uranium) }\end{array}$ & SGR-2 & 09/14/2002 & $1,219.2-2,035.1$ & $4,000-6,677$ \\
\hline
\end{tabular}




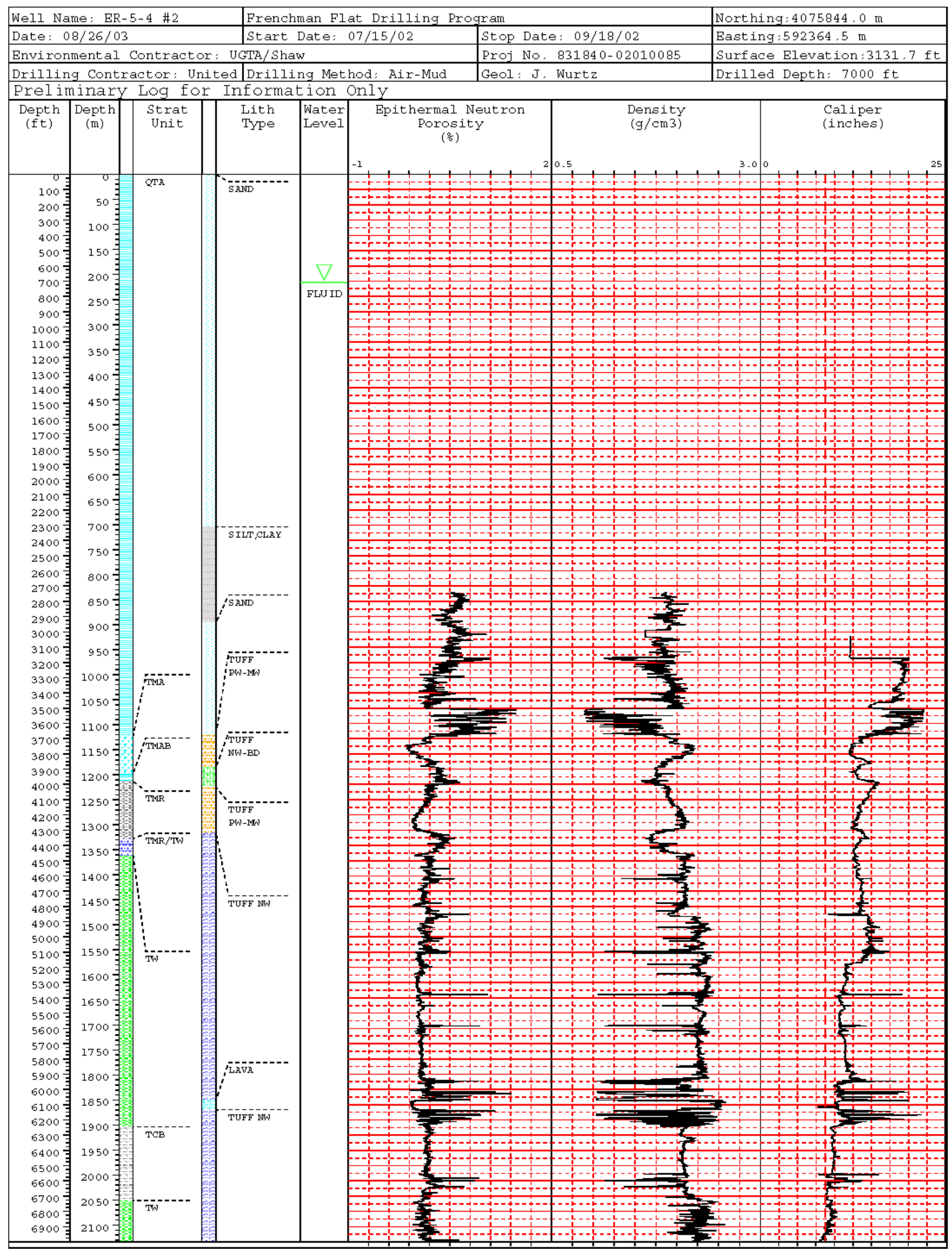

D-2-2 


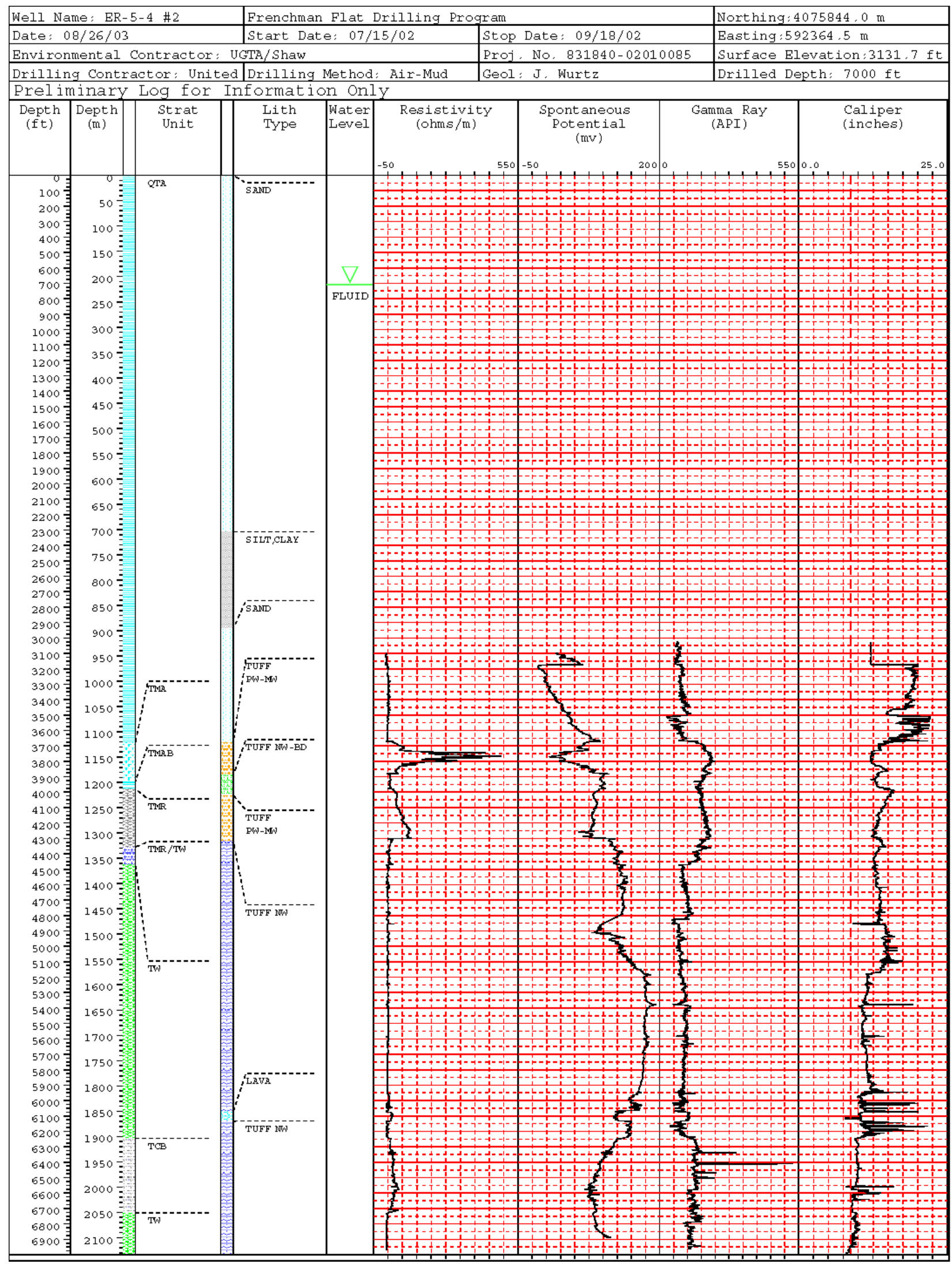

D-2-3 


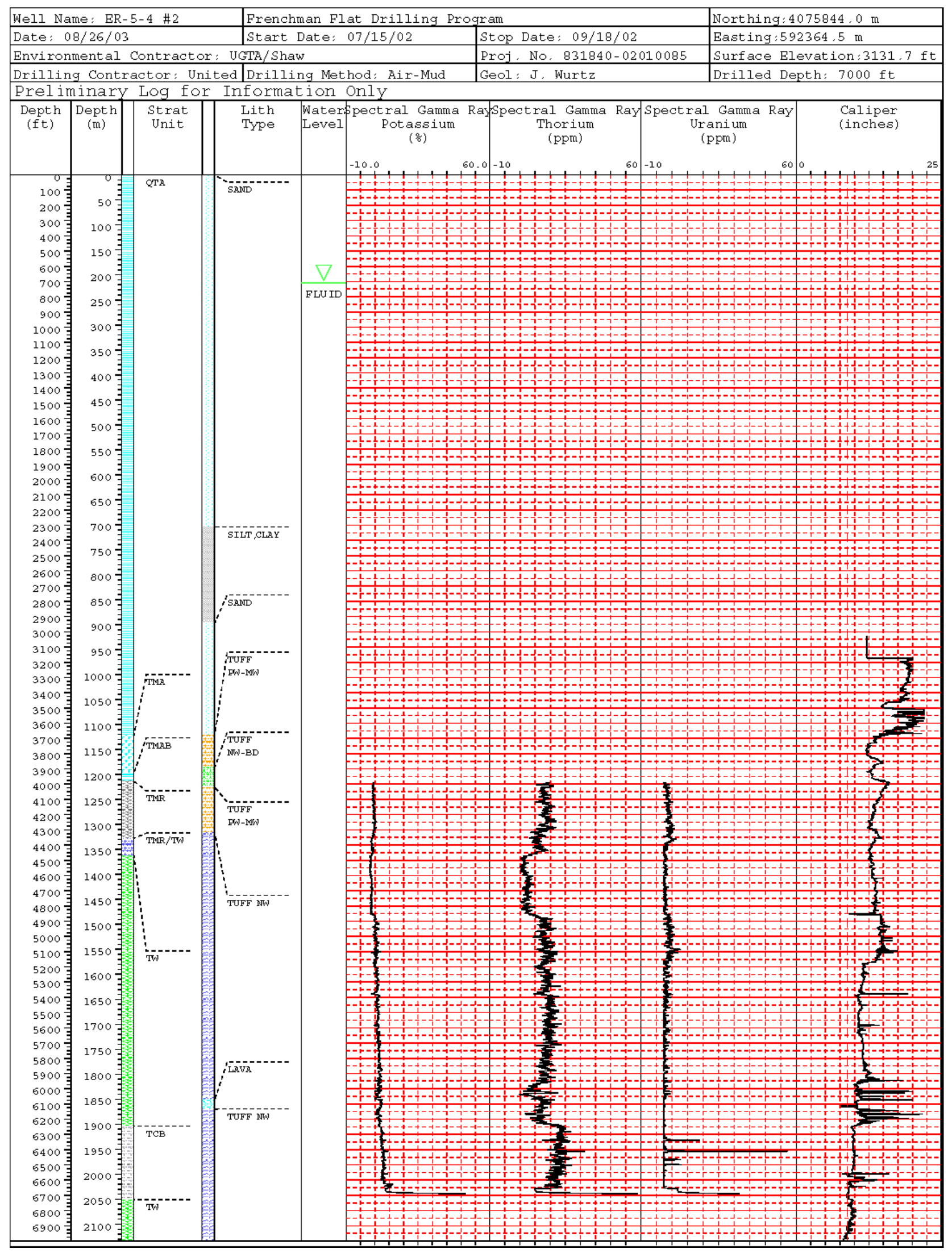




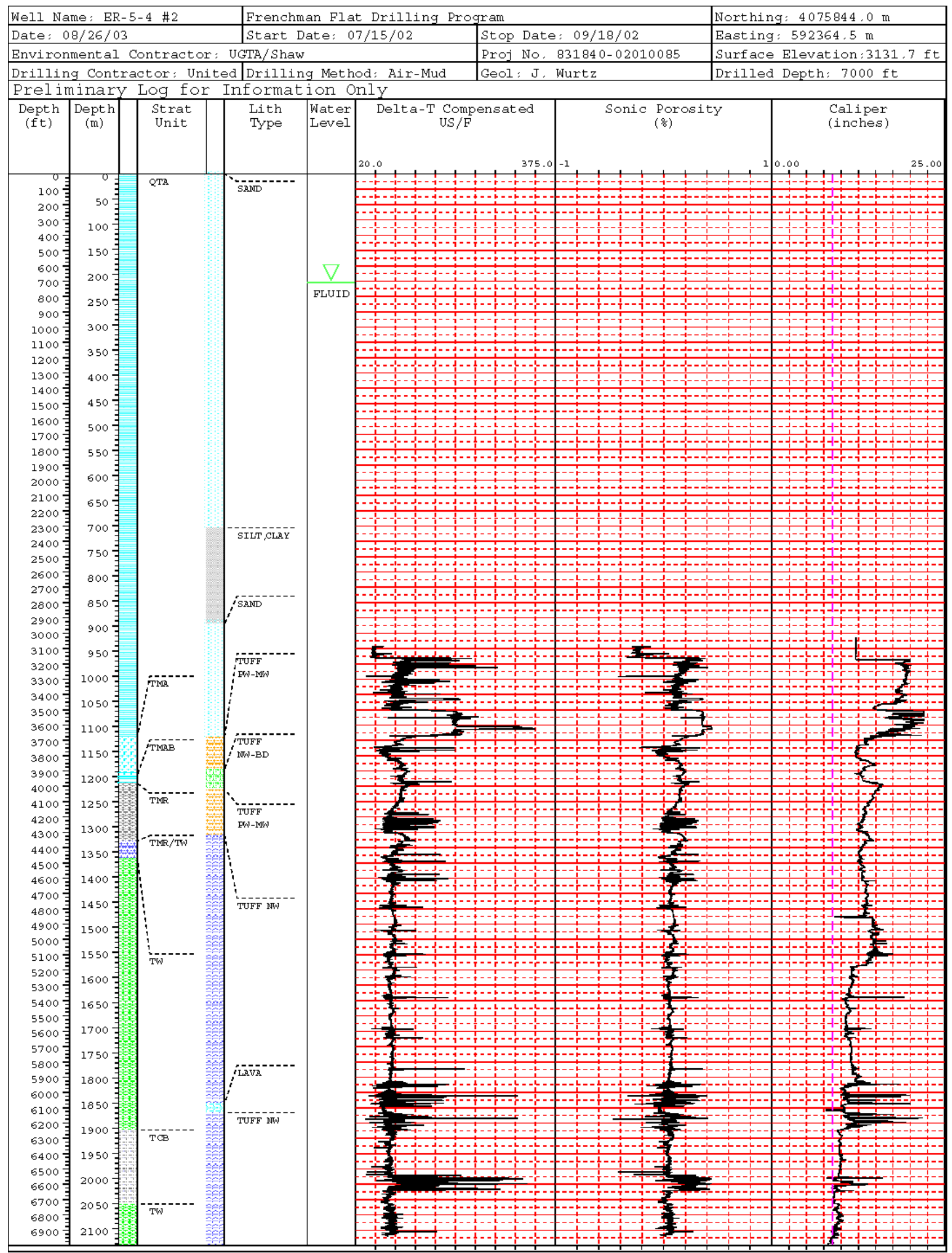


This page intentionally left blank.

D-2-6 


\section{Distribution List}

\section{$\underline{\text { Copies }}$}

R. M. Bangerter

3

U.S. Department of Energy

National Nuclear Security Administration

Nevada Site Office

Environmental Restoration Division

P.O. Box 98518, M/S 505

Las Vegas, NV 89193-8518

U.S. Department of Energy

National Nuclear Security Administration

Nevada Site Office

Technical Library

P.O. Box 98518, M/S 505

Las Vegas, NV 89193-8518

U.S. Department of Energy

2 (electronic)

National Nuclear Security Administration

Nevada Site Office

Nuclear Testing Archive

Public Reading Facility

P.O. Box 98518, M/S 400

Las Vegas, NV 89193-8518

U.S. Department of Energy

1 (electronic)

Office of Scientific and Technical Information

P.O. Box 62

Oak Ridge, TN 37831-0062

K. A. Hoar, Director

U.S. Department of Energy

National Nuclear Security Administration

Nevada Site Office

Environment, Safety and Health Division

P.O. Box 98518, M/S 505

Las Vegas, NV 89193-8518

P. K. Ortego

Bechtel Nevada

P.O. Box 98521, MS NLV082

Las Vegas, NV 89193-8521 


\section{Distribution List (continued)}

\section{$\underline{\text { Copies }}$}

John McCord

UGTA Project Manager

Stoller-Navarro

7710 West Cheyenne Ave.

Building 3

Las Vegas, NV 89129

Stoller-Navarro Library

Stoller-Navarro

7710 West Cheyenne Ave.

Building 3

Las Vegas, NV 89129

W. L. Hawkins

Los Alamos National Laboratory

P. O. Box 1663

Los Alamos, NM 87545-1663

G. A. Pawloski

1

Lawrence Livermore National Laboratory

P. O. Box 808

Livermore, CA 94551-0808

T. P. Rose

Lawrence Livermore National Laboratory

P. O. Box 808

Livermore, CA 94551-0808

B. K. Thompson

1

DOE/USGS Cooperative Program Manager

U.S. Geological Survey

Water Resources Division

6770 South Paradise Road

Las Vegas, NV 89119-3721

C. E. Russell

Desert Research Institute

755 East Flamingo Road

P.O. Box 19040

Las Vegas, NV 89119-7363 